\title{
WestVirginiaUniversity
}

THE RESEARCH REPOSITORY @ WVU

Graduate Theses, Dissertations, and Problem Reports

2009

\section{The fearful face and beyond: fMRI studies of the human amygdala}

Jillian E. Hardee

West Virginia University

Follow this and additional works at: https://researchrepository.wvu.edu/etd

\section{Recommended Citation}

Hardee, Jillian E., "The fearful face and beyond: fMRI studies of the human amygdala" (2009). Graduate Theses, Dissertations, and Problem Reports. 2933.

https://researchrepository.wvu.edu/etd/2933

This Dissertation is protected by copyright and/or related rights. It has been brought to you by the The Research Repository @ WVU with permission from the rights-holder(s). You are free to use this Dissertation in any way that is permitted by the copyright and related rights legislation that applies to your use. For other uses you must obtain permission from the rights-holder(s) directly, unless additional rights are indicated by a Creative Commons license in the record and/ or on the work itself. This Dissertation has been accepted for inclusion in WVU Graduate Theses, Dissertations, and Problem Reports collection by an authorized administrator of The Research Repository @ WVU.

For more information, please contact researchrepository@mail.wvu.edu. 


\title{
The Fearful Face and Beyond: fMRI Studies of the Human Amygdala
}

\author{
Jillian E. Hardee \\ Dissertation submitted to the \\ School of Medicine \\ at West Virginia University \\ in partial fulfillment of the requirements \\ for the degree of \\ Doctor of Philosophy \\ in \\ Neuroscience \\ Aina Puce, Ph.D, Advisor \\ James Lewis, Ph.D, Chair \\ Marc Haut, Ph.D, A.B.P.P. \\ Janine Mendola, Ph.D \\ Ben Ramsden, Ph.D \\ Department of Neurobiology and Anatomy \\ Department of Radiology \\ Morgantown, West Virginia \\ 2009
}

Keywords: Amygdala; fMRI; Emotion; Face Processing 


\section{ABSTRACT \\ The Fearful Face and Beyond: fMRI Studies of the Human Amygdala}

Jillian E. Hardee

The amygdala has been labeled as a "detector of threat", evidenced by its heightened response to fearful faces in human neuroimaging studies. A critical element of the fearful face is an increase in eye white area, hypothesized to be crucial for the rapid detection of fear in another's face. Yet other facial expressions can also increase eye white area in a manner that is similar (a lateral shift in gaze) or identical (surprise) to fear. It is unknown if the amygdala can differentiate between these types of increases in eye white area and those that are specifically associated with fear when using only the eye region of the face. Furthermore, whether the fearful mouth can elicit an amygdala response when shown in isolation is unknown. Using functional magnetic resonance imaging, we found that the amygdala responded robustly to fearful eyes as well as eye stimuli that were ambiguous in nature. The fearful mouth, on the other hand, was unable to generate a significant response from the amygdala, however the happy condition elicited a slight response from the right amygdala, most likely due to the visual salience of the smile. We also observed a functional laterality between the two amygdalae in that the left amygdala responded only to fearful eyes while the right amygdala activated to any change in the eyes; the same laterality was also evident when eye stimuli were ambiguous in nature indicating that the left amygdala is more tuned to detect fear in the eyes while the right amygdala acts as a general detector of eye changes. This lends more evidence to the existence of parallel mechanisms for processing visual threat. Together, our results indicate that while the amygdala is primarily a detector of fearful faces, it has evolved to respond to other facial expressions that are also behaviorally relevant or potentially threatening to the viewer. 


\section{Acknowledgements}

I would first and foremost like to thank Aina Puce for taking me into her lab and introducing me to the intriguing world of neuroimaging. I also thank James Lewis for stepping in as the auxiliary committee chair for the last year of my doctoral work. I deeply appreciate both of your efforts in helping me shape my dissertation. I would also like to thank the members of my committee for their contributions as well as for their patience. To Janine Mendola and Ben Ramsden, thank you for remaining committed to my work even after you moved on to other universities. And finally, thank you to Marc Haut for filling a spot on my committee after we had to do some rearranging to meet university requirements - and also for trusting me to watch your dogs even though I sometimes took photos of them wearing funny hats.

Thank you to my husband Ben Shade for putting up with me, even when I didn't listen to your advice on how to use Word effectively. You were right. But I still hate math!

I would not have made it to the end of my graduate career without all the members of the CAI, both past and present. I am exceedingly appreciative for all the support, assistance, and/or the time you spent in the scanner being a subject. So here's a gigantic thank you to Julie Brefczynski-Lewis, Mary Pettit, Linda Shuster, Susan Lemeiux, Bill Talkington, Chris Frum, and Jim Thompson.

To my co-conspirator Amy Prostko, there are not enough words to express how grateful I am that we are friends. You never cease to make me laugh. If you ever called and asked "can you bring me my chapstick?" I would do it.

To all the subjects who participated in my studies and let me pay them in chocolate when research money was tight, you are awesome.

Finally, thank you to everyone and everything that helped me get through graduate school: Michelle Saylor, Fitz, the penguins, Leor Zellner, the amygdala, and Craigory Hardee. To radiology, thanks for all the exercise and heavy lifting. 


\section{Table of Contents}

Acknowledgements .................................................................................................................................... iii

Table of Contents ......................................................................................................................................... iv

List of Figures........................................................................................................................................... vii

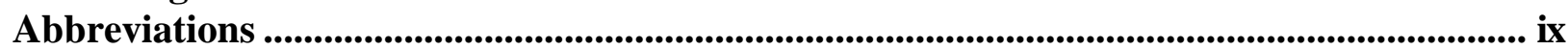

CHAPTER 1: Introduction and Literature Review .................................................................... 1

1.1 Human Socialness ............................................................................................................. 2

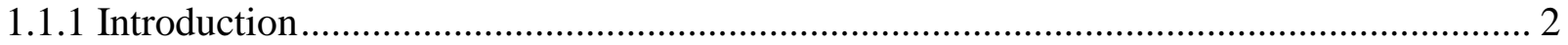

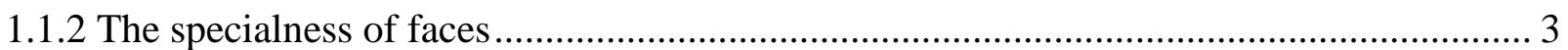

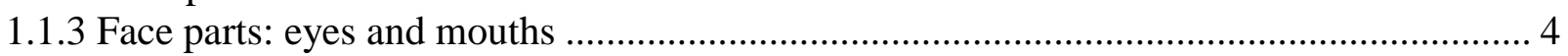

1.2 The Amygdala ........................................................................................................................................ 7

1.2.1 History of amygdala studies and overview of its functional roles .................................... 7

1.2.2 Amygdala anatomy and connections ......................................................................... 9

1.2.3 The role of the amygdala in visual processing............................................................ 10

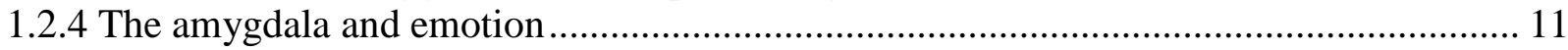

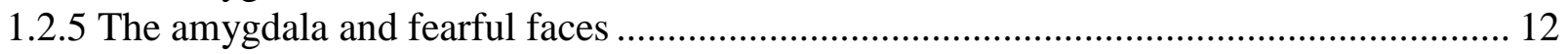

1.3 Amygdala Dysfunction ................................................................................................................ 14

1.3.1 Amygdala involvement in psychological and social disorders ....................................... 14

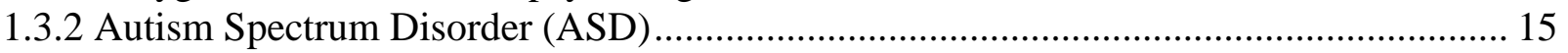

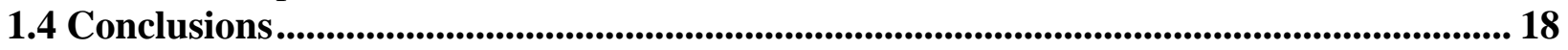

1.5 Figures..................................................................................................................................................................... 19

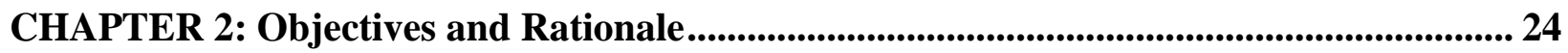

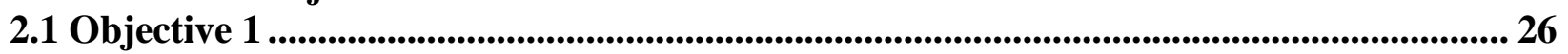

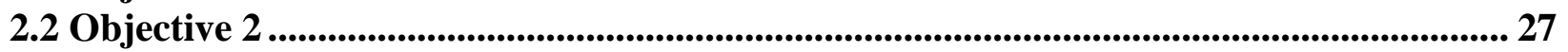

CHAPTER 3: Experiment 1 - Preliminary Experimental Data: Masking Eye Gaze Changes

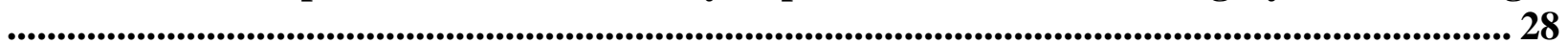

3.1 Introduction and Rationale ......................................................................................... 29

3.2 Methods............................................................................................................................................................. 30

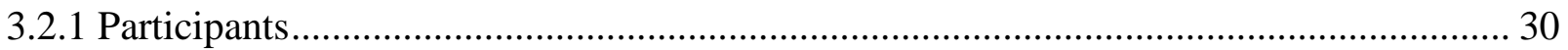

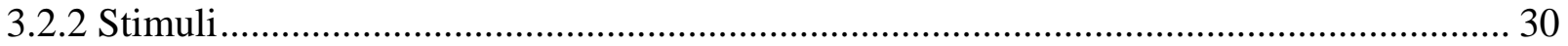

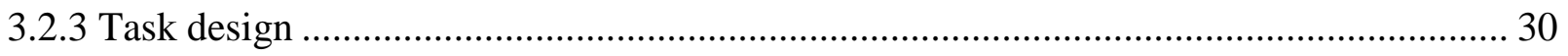

3.3 Results ..................................................................................................................................................... 32

3.4 Discussion......................................................................................................................................... 34

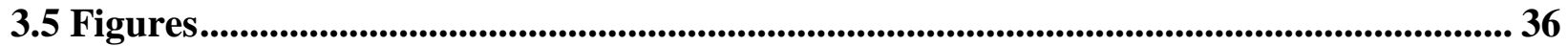

CHAPTER 4: Experiment 2 - The Left Amygdala Knows Fear: Laterality in the Amygdala Response to Fearful Eyes........................................................................................................................ 37

4.1 Abstract.................................................................................................................................... 38

4.2 Introduction.............................................................................................................................. 39

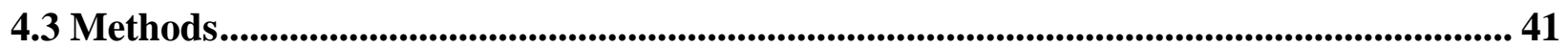

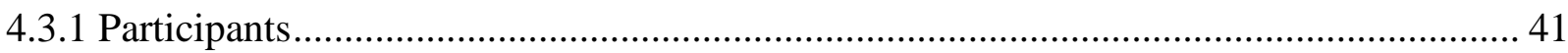

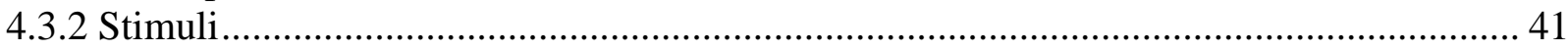




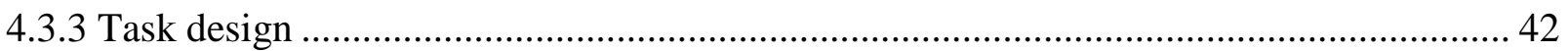

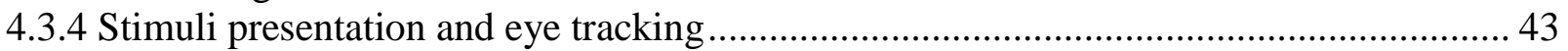

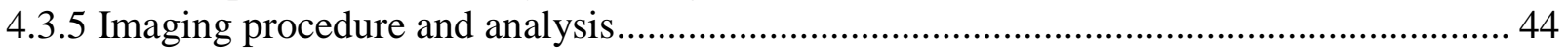

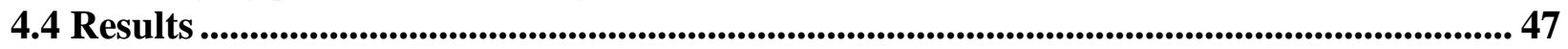

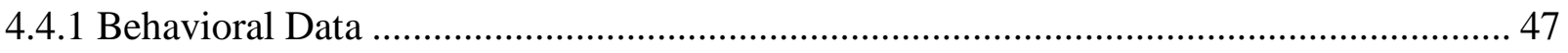

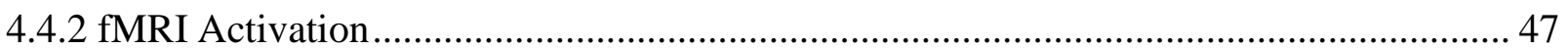

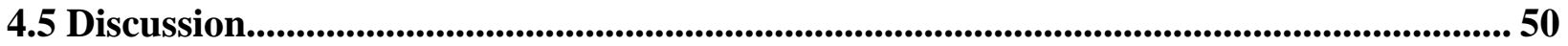

4.6 Figures and Tables....................................................................................................................................... 54

CHAPTER 5: Experiment 3 - Amygdala Activation to Emotional Eyes and Eyebrows ..... 61

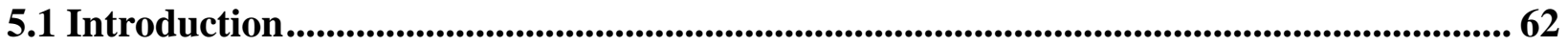

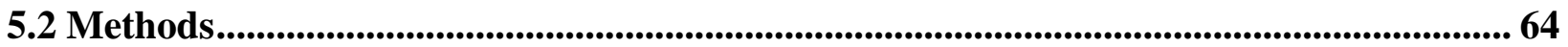

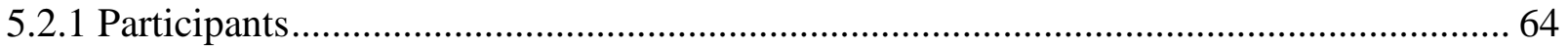

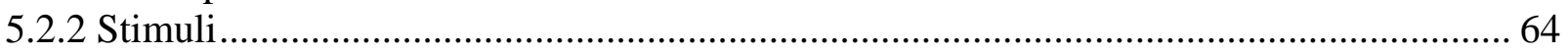

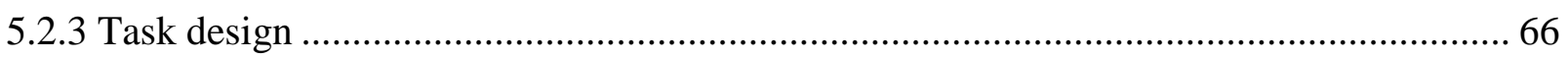

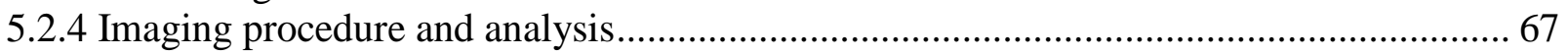

5.3 Results ............................................................................................................................ 69

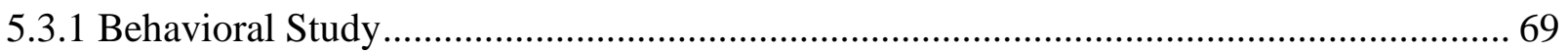

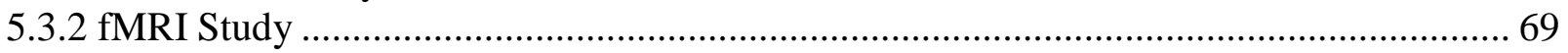

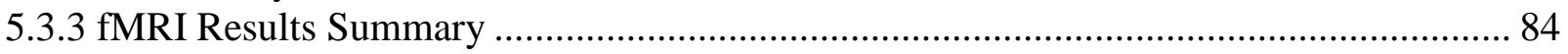

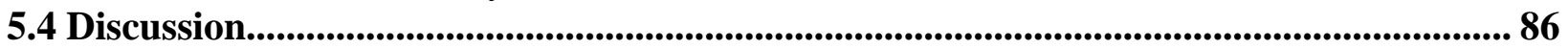

5.5 Figures............................................................................................................................................ 90

CHAPTER 6: Experiment 4 - Amygdala Activation to Emotional Mouths............................ 98

6.1 Introduction............................................................................................................................... 100

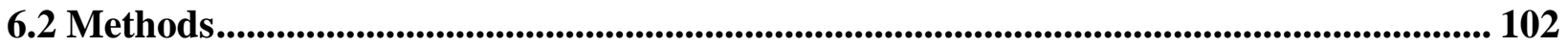

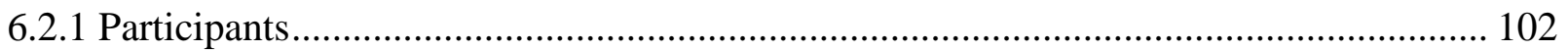

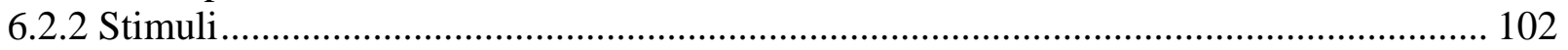

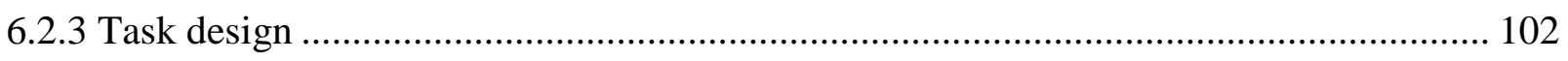

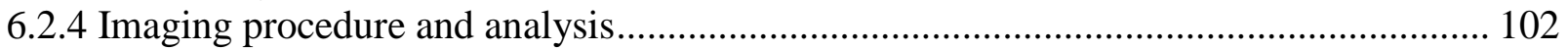

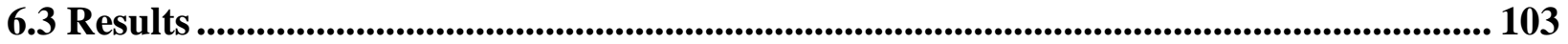

6.3.1 Behavioral Study ................................................................................................. 103

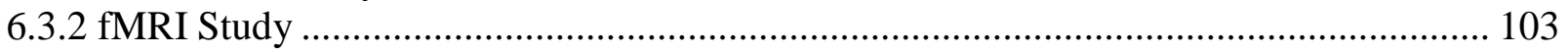

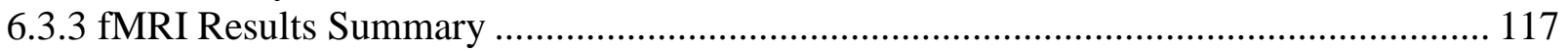

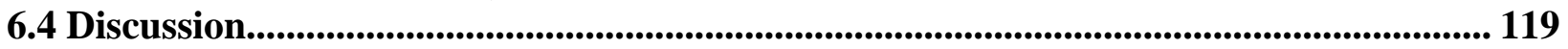

6.5 Figures.................................................................................................................................................. 122

CHAPTER 7: General Discussion and Conclusions.......................................................... 129

7.1 Discussion of Amygdala Findings............................................................................................ 130

7.1.1 Summary of amygdala results ............................................................................... 130

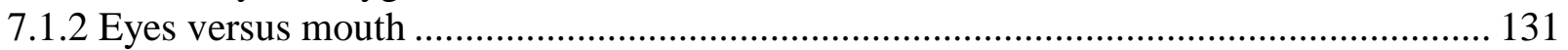

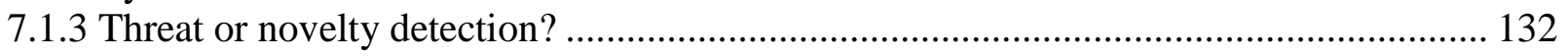

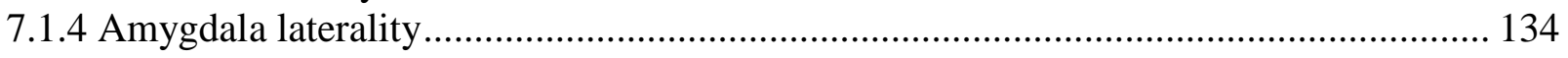

7.1.5 Other considerations ............................................................................................. 139

7.2 Discussion of Significant Findings in Other Brain Regions ................................................ 140

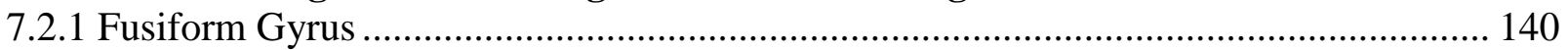


7.2.2 Intraparietal Sulcus

7.2.3 Activation for brain regions that did not differentiate per stimulus condition .............. 145

7.3 General Summary ..................................................................................................... 147

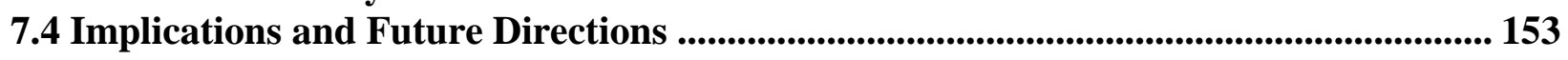

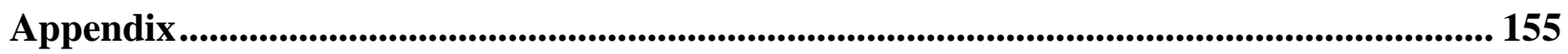

A.1 Functional Magnetic Resonance Imaging .............................................................................. 156

A.2 PickAtlas Volumes of Interest................................................................................................... 159

A.3 Worsley Correction Calculation ...................................................................................... 164

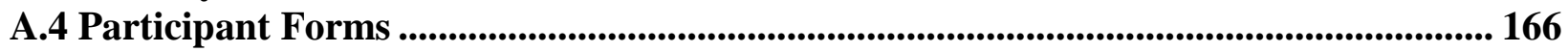

A.5 Determining Optimal Scanning Parameters ........................................................................... 170

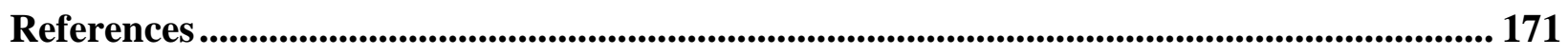

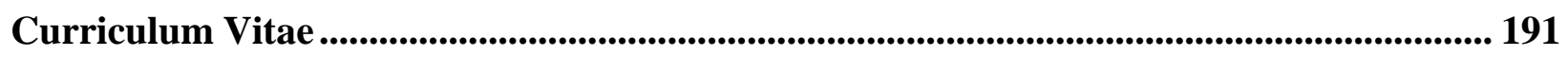




\section{List of Figures}

\section{Chapter 1}

Figure 1-1 (1) Anatomical image of human brain ......................................................... 19

Figure 1-2 (2) Areas active in face processing ..................................................................... 20

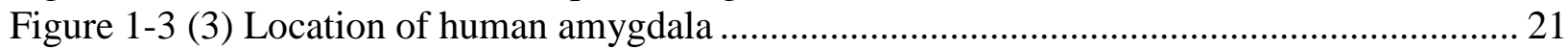

Figure 1-4 (4) Outputs from amygdala nuclei ............................................................... 22

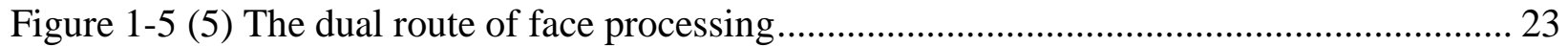

Figure 1-6 (6) Eye fixations made during presentations of faces .......................................... 24

\section{Chapter 3}

Figure 3-1 (7) Example of single event trial (Experiment 1).................................................. 36

\section{Chapter 4}

Figure 4-1 (8) Representative stimuli for each condition (Experiment 2) ................................ 54

Figure 4-2 (9) Mean percent eye white area change from neutral ............................................. 55

Figure 4-3 (10) Example of a single trial (Experiment 2) ................................................... 56

Figure 4-4 (11) Amygdala group activation patterns and percent MR signal change ................. 57

Figure 4-5 (12) Percent MR signal change for FG and IPS ................................................ 58

Figure 4-6 (13) Percent MR signal change for STS and Inf/OFC ........................................ 59

\section{Chapter 5}

Figure 5-1 (14) Representative stimuli for each condition (Experiment 3) ............................. 90

Figure 5-2 (15) Example of eyebrow configuration changes ................................................ 91

Figure 5-3 (16) Average pixel change for each condition from neutral ................................. 92

Figure 5-4 (17) BOLD activation in the amygdala .......................................................... 93

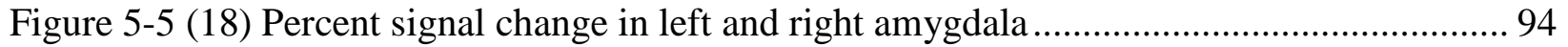

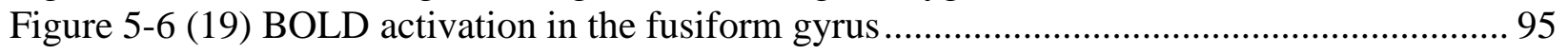

Figure 5-7 (20) BOLD activation within the temporal VOI ................................................ 96

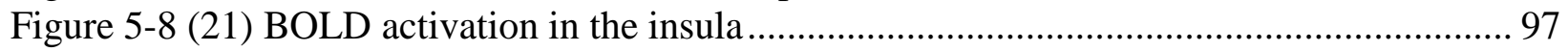

Figure 5-9 (22) BOLD activation within the parietal VOI .................................................. 98

\section{Chapter 6}

Figure 6-1 (23) Representative stimuli for each condition (Experiment 4)........................... 122

Figure 6-2 (24) BOLD activation in the amygdala ....................................................... 123

Figure 6-3 (25) Percent signal change in the left and right amygdala .................................. 124

Figure 6-4 (26) BOLD activation in the fusiform gyrus ................................................... 125

Figure 6-5 (27) BOLD activation within the temporal VOI ............................................. 126

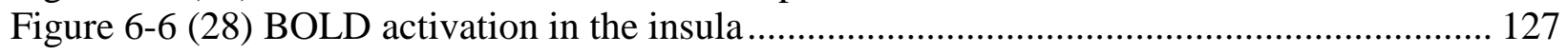

Figure 6-7 (29) BOLD activation within the parietal VOI .............................................. 128 


\section{Chapter 7}

Figure 7-1 (30) Percent signal change summary for Experiment 2 for amygdala, FG, IPS....... 147

Figure 7-2 (31) Percent signal change summary for Experiment 3 for amygdala, FG, IPS....... 148

Figure 7-3 (32) Percent signal change summary for Experiment 4 for amygdala, FG, IPS....... 149

\section{Appendix}

Figure A-1 (33) PickAtlas: amygdala VOI ............................................................................. 159

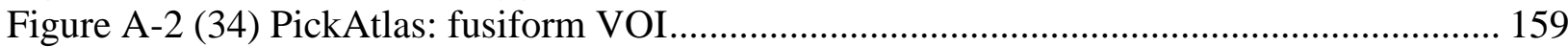

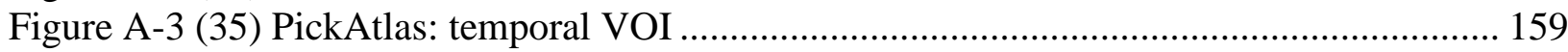

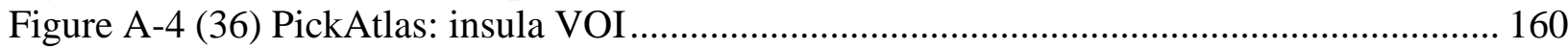

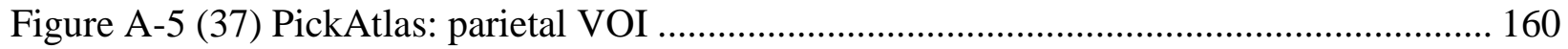

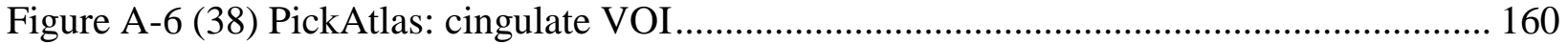

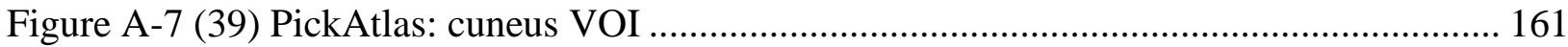

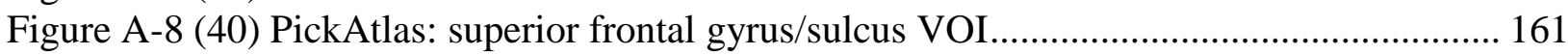

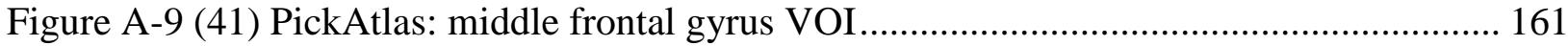

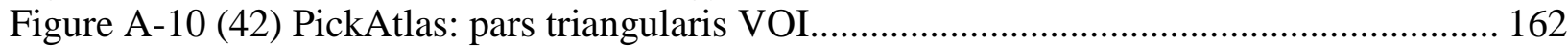

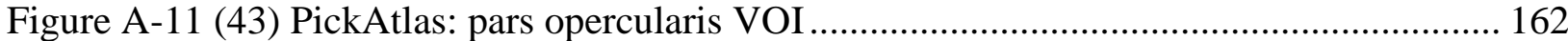

Figure A-12 (44) PickAtlas: supraorbital sulcus VOI ........................................................... 162

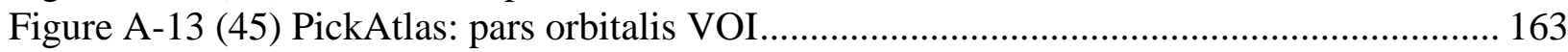

Figure A-14 (46) PickAtlas: medial superior frontal gyrus VOI ................................................ 163 


\section{Abbreviations}

\begin{tabular}{ll} 
ANOVA & analysis of variance \\
ASD & autism spectrum disorder \\
BOLD & blood oxygen level dependent \\
BPD & borderline personality disorder \\
EWA & eye white area \\
FFA & fusiform face area \\
FG & fusiform gyrus \\
fMRI & functional magnetic resonance imaging \\
FOV & field of view \\
FWHM & full width half maximum \\
Inf/OFC & inferior and orbito frontal cortex \\
IPS & intraparietal sulcus \\
L & left \\
MNI & Montreal Neurological Institute \\
MR & magnetic resonance \\
ms & millisecond(s) \\
OFA & occipital face area \\
PET & positron emission tomography \\
PTSD & post-traumatic stress disorder \\
R & right \\
RF & radio frequency \\
SPGR & spoiled gradient recall \\
STS & superior temporal sulcus \\
T & tesla \\
TE & echo time; in monkeys, refers to inferotemporal cortex \\
TR & scan repeat time \\
VOI & volumes of interest \\
\hline
\end{tabular}


CHAPTER 1: Introduction and Literature Review 
Social interaction in humans relies on more than just verbal communication: gestures, expressions, body posture, and other forms of nonverbal communication are extremely important for relating to one another. This chapter covers key concepts in how the human brain recognizes and processes information from the face - specifically the eyes and mouth - as well as how a specific structure of the brain called the amygdala detects and uses emotional information from the face and face parts. The background information presented here will provide a foundation for understanding the experiments covered in later chapters.

\subsection{Human Socialness}

\subsubsection{Introduction}

Humans are one of the most social animals and social interactions are a major driving force of human evolution (Humphrey, 1976). Because of this high degree of social ability and our dependency on it, humans must have the capability to interpret the emotions and actions of others, thus we have an intricate structure of communication that uses vocalizations, gestures, language, and facial expressions. Humans can also infer the mental states of others through body expressions, tone and inflection of voice, and prior experiences.

Faces are particularly important as they lend a wealth of information with respect to identity, sex, mood, age, race, and direction of attention. Through faces we can also read the dispositions and emotions of those we interact with. Despite the canonical universal T-shaped representation of faces - two eyes, a nose, and a mouth - humans are able to discriminate between many different faces, store this information, and use it for later recognition. Facial expressions are the most obvious indicator of an individual's emotional state. In his 1898 book The Expression of the Emotions in Man and Animals, Charles Darwin stated his belief that the primary emotions conveyed by the face are universal and that these forms of nonverbal communication are species, not culturally, specific. In 1969, Ekman et al. hypothesized that the universality of certain emotions are found in the characteristic patterns of the facial muscles. Along these lines, there is evidence to show that, across cultures, consistencies exist in the emotional meanings of six basic emotional expressions: happiness, surprise, fear, sadness, anger, and disgust (Ekman et al., 1970). 


\subsubsection{The specialness of faces}

An abundance of neurological evidence validates the idea that faces are very informative and important. Patients with acquired prosopagnosia lose their ability to recognize faces after brain damage (Barton, 2003) but are still able to identify individuals through vocal and/or verbal cues. The face-inversion effect, which exemplifies the differences in how objects and faces are processed, demonstrates that when face and object stimuli are inverted the performance decrement for faces is much greater than non-face stimuli (Yin, 1969). Subjects are also better at discriminating which face parts appeared in a previously shown face when features are shown in the context of the whole face as opposed to being shown in isolation (Tanaka and Farah, 1993). Moreover, electrophysiological studies using scalp electrodes reliably measure face-selective responses that occur 170ms after stimulus onset (Bentin et al., 1996; Jeffreys, 1996); this is higher for faces compared to non-face objects, thus demonstrating face selectivity (Allison et al., 1999). Because electrophysiological studies using scalp electrodes are limited in spatial resolution, experiments employing subdural electrode measurements in patients with epilepsy indicated strongly face-selective responses in distinct portions of the temporal lobe (Allison et al., 1994; 1999). Further studies using positron emission tomography (PET) verified activation of the ventral visual pathway, in particular the fusiform gyrus, to various experimental tasks involving faces (Haxby et al., 1994; Andreasen et al., 1996; Clark et al., 1996).

With the advent of functional magnetic resonance imaging (fMRI) in the early 1990s (see Appendix Section A.1 for more details on fMRI), the specificity of the fusiform gyrus to face stimuli was able to be studied more closely. These initial experiments established that certain regions of the fusiform respond more strongly to faces than to textures and letterstrings (Puce et al., 1996), flowers (McCarthy et al., 1997), houses, everyday objects, and hands (Kanwisher et al., 1997), and thus this area (located on the lateral side of the mid-fusiform gyrus) was deemed the 'fusiform face area', or FFA (Kanwisher et al., 1997; see Figure 1-1). While the FFA is the most consistent and robust face-selective activator, it is important to note that regions of the superior temporal sulcus (STS) and occipital lobe (named 'occipital face area', or OFA) are also face-sensitive yet do not activate as reliably across subjects. A study by Haxby et al. (2001) indicates that the FFA and OFA are mostly involved in distinguishing between individual faces while the STS appears to extract other elements including gaze direction and emotional 
expression. Additional evidence suggests that FFA, but not STS, activation readily correlates with successful face detection (Andrews and Schluppeck, 2004; Grill-Spector et al., 2004), again showing that the STS may not be sensitive to face identity information (Hoffman and Haxby, 2000; Grill-Spector et al., 2004). The STS has more generally been implicated in the analysis of biological motion (Bonda et al., 1996), including gaze and mouth movements (Puce et al., 1998; Grossman et al., 2000; Kourtzi \& Kanwisher, 2000; Thompson et al., 2005).

While it is accepted that the FFA is involved in the processing of faces, there is debate over the exact functional organization of the face processing system in how each component works, what it does, and how this works in reality. However, there is substantial evidence illustrating different roles between the left and right STS and their involvement in face processing (Puce et al., 2000), the part that frontal areas play processing faces (Grady et al., 2000; Marinkovic et al., 2000; Vignal et al., 2000), posterior mechanisms that encode the structure of faces (George et al., 1999; Bentin and Deouell, 2000), and how distinctive systems are involved in the production and perception of emotional expressions and responses (Calder et al., 2000; Breen et al., 2000). Beyond this, the actual function of the FFA is still being debated. Is it simply involved in the detection of faces (Tong et al., 2000), the structural encoding of faces (George et al., 1999) or the subordinate-level categorization of nonface objects (Gauthier et al., 2000)? Several groups have developed a distributed representation model of face processing (Bruce and Young, 1986; Haxby et al., 1996; Hoffman and Haxby, 2000; de Gelder et al., 2003) in which attributes of a face (such as identity) are processed by the fusiform gyrus (FG) and inferior occipital gyrus, gaze direction and the recognition of action are processed by the STS, and expression and emotion are processed via the orbitofrontal cortex, the amygdala, the anterior cingulate, and the premotor cortex (Figure 1-2).

\subsubsection{Face parts: eyes and mouths}

Often, certain parts of the face are more important in the processing of particular facial expressions and emotions. The eyes are especially adept at transmitting information beneficial for social interaction. The human eye, with its dark iris and large extent of visible sclera, makes interpreting eye gaze direction and emotion easier (Emery, 2000), even at a distance. Of all features, the eye area is the facial region where most attention is directed and it significantly aids with the identification of other individuals (Sadr et al., 2003), in determining gender (Schyns et 
al., 2002), and perceiving emotion - especially fear (Whalen et al., 2004; Adolphs et al., 2005). Even non-human primates such as baboons and rhesus monkeys look at the eye region more than the nose and mouth; other primates have coloration around the eyes or colored brow ridges highlighting and directing attention to their eyes (Emery, 2000). The perception of gaze is evident early on in human life, as newborns show a preference for looking at faces with open rather than closed eyes (Batki et al., 2000), and their attention to faces extends longer to those individuals directly gazing at them versus looking away (Farroni et al., 2002). The direction of eye gaze can point the attention of observers to certain objects or places in the surrounding environment, and can indicate their thoughts and goals. Following the gaze of another can also help us learn about our environment; rhesus macaques that were not previously afraid of snakes learned to fear them after observing the direction of their parents' gaze paired with a fearful response when snakes were near (Mineka et al., 1984). Furthermore, the eyes can also enable the characterization of certain emotions: fear and surprise are epitomized by a widening of the eyes and an increase in eye white visibility while eyes looking downward can signify sadness.

Gaze processing has been shown to activate the FG, STS, intraparietal sulcus (IPS), and amygdala (Hoffman and Haxby, 2000; George et al., 2001; Pelphrey et al., 2003). Changes in perceived gaze direction have been shown to alter FG activity (see Itier and Batty, 2009, for a review), while perceiving faces using only eye stimuli elicits similar but weaker activation of the FFA compared to eyes presented in the connotation of the whole face (Watanabe et al., 1999a; Watanabe et al., 1999b; Tong et al., 2000). The STS activates to faces with moving eyes and mouths - but not other moving face parts (Puce et al., 1998), and to changes in eye gaze (Hoffman and Haxby, 2000). The ability to follow gaze may depend on connectivity between the STS and the IPS, another region also shown to be responsive to different aspects of gaze-related activity. The amygdala as well plays a large role in the detection and recognition of different eye information, and this is covered more specifically in Section 1.2.

Like the eyes, the mouth also transmits social information. Aside from its obvious involvement in speech, the mouth enables the identification of certain expressions and emotions. Happiness is almost entirely characterized by the mouth region, as a smile is nearly recognized universally (Schyns et al., 2002). The mouth itself transmits different types of movement, and the STS has been shown to activate differentially to linguistic (Calvert et al., 1997; Sams et al., 1997) and non-linguistic (Puce et al., 1999; 2003) mouth movements. But while many studies 
have looked at how the mouth activates brain regions with respect to language (i.e. speech perception, lip-reading) and mouth movements in general, whether mouths conveying specific emotions can modulate face processing is not well studied. Recent data indicates that in virtually all expressions the eyes are more informative than the mouth (Adolphs et al., 2005; Stephan et al., 2006), demonstrating that the mouth may play an accessory role in the recognition of most emotions. 


\subsection{The Amygdala}

\subsubsection{History of amygdala studies and overview of its functional roles}

The amygdala consists of a group of nuclei located deep in the medial temporal lobes of the brain in many complex vertebrates, including humans. The amygdala is a structure in the brain well known for its involvement in and response to emotional and social stimuli. In particular, the amygdala plays a large role in detecting potential threats in the surrounding environment. Because the amygdala is part of the emotion network and is highly interconnected with many subcortical and cortical brain regions, dysfunction or damage of the amygdala can lead to disruptions in emotional and social processing. More about the anatomy of the amygdala will be covered in Section 1.2.2.

Numerous studies that aided in understanding the amygdala were first performed using rats, and this led to greater understanding of the relationship between the amygdala and fearful behavior. For example, lesioned rats showed less trepidation to new foods than non-lesioned rats (Rolls and Rolls, 1973). Other experiments with rodents also contributed greatly to understanding the neural mechanisms behind fear conditioning: unilateral amygdala damage in rats reduced conditioned freezing responses to aversive stimuli while bilateral lesions abolished freezing behavior almost entirely (LaBar and LeDoux, 1996). Lesion studies in primates have furthered the understanding of the amygdala by elucidating how it is involved in emotional and social cognition. One such experiment by Kluver and Bucy (1939) demonstrated the importance of the amygdala in the recognition of threat. Bilateral removal of the temporal lobes - including the amygdala and hippocampus - in monkeys caused a lack of fearful behavior in that, without hesitation, the monkeys would approach objects that were previously seen as threatening. In addition, marked behavioral changes were also noted as the lesioned monkeys would fight with the more dominant monkeys in the hierarchy. Later, Zola-Morgan et al. (1991) reported that lesions to the amygdala, but not the hippocampus, caused these behavioral changes in monkeys indicating that the amygdala is a structure necessary for the detection of threat.

Amygdala damage in humans has also contributed to the understanding of amygdala function however, although unlike lesion studies in monkeys, the size, extent, and location of the lesions cannot be controlled. Patients with injury to the amygdala are often impaired in correctly identifying the facial expressions of others in both static and dynamic stimuli; this impairment 
extends most heavily to fearful faces (Adolphs et al., 1994). The removal of the amygdala can also impair memory for faces and the recognition of emotional expressions (Young et al., 1995). An example demonstrating this deficiency is the case of patient SM, who had rare bilateral damage that was confined only to the amygdala (Adolphs \& Tranel, 1999). SM was unable to identify fearful emotions in human faces, and could not draw a fearful face even though other emotions such as happiness, sadness, anger, and disgust could readily be identified and drawn. Additional studies by this group show that SM was impaired at perceiving social and emotional information, exhibits abnormal conditioned fear responses, and was atypically trusting and friendly of others (Bechara et al., 1995; Adolphs et al., 1998). SM also appears to be unable to use information from the eyes when making judgments about emotion, hypothesized to result from a lack of freely fixating on the eye region of faces (Adolphs et al., 2005). Patients with bilateral amygdala damage are more accurate (compared to controls) at identifying emotional scenes when faces are obscured, indicating that the amygdala may be particularly important for recognizing emotion from faces but generally not from scenes and events (Adolphs and Tranel, 2003). Amygdala lesions can also affect distant brain regions, as shown by Vuilleumier et al. (2004). Twenty-six patients with both bilateral and unilateral amygdala lesions were shown pictures of fearful and neutral faces, and even though all had intact fusiform and occipital cortices, they did not show increased activation in these regions to the face stimuli the way control subjects did. Studies of individuals with unilateral amygdala lesions had hinted at a functional laterality in the amygdala with respect to processing fearful faces (amygdala laterality is covered further in Chapter 4); in patients with mesial temporal lobe damage, those with rightsided lesions were impaired in their ability to recognize fearful expressions (Benuzzi et al., 2004). Conversely, Palermo et al. (2007) did not find this impairment for fear recognition in patients with right-sided amygdala lesions but did find that patients with left-sided lesions had trouble reliably recognizing fearful faces (Palermo et al., 2007). Together both unilateral and bilateral amygdala studies strongly suggest that the human amygdala is selective for the recognition of fear in faces.

Other data suggests that the human amygdala plays a role in detecting and processing positive emotions (Rolls, 2000; Baxter and Murray, 2002; Holland and Gallagher, 2004) and also can be engaged by signals of a social nature (Barton and Aggleton, 2000; Adolphs 2003a,b). In addition, there have been reports of hemispheric differences in amygdala function (Markowitcsh, 
1998; Morris et al., 1998a; Wright et al., 2001; Glascher and Adolphs, 2003) as well as a modulation of response output based on the sensory modality of stimulus input (Markowitcsh, 1998, Barton and Aggleton, 2000).

The amygdala also has a well-defined role in classical fear conditioning paradigms, where an organism acquires a conditioned fear response to a previously neutral stimulus through the direct pairing of this with a naturally aversive event, or unconditioned stimulus. Here, the amygdala has proven to be critical in the acquisition, storage, and expression of fear in both human (LaBar et al., 1995; LaBar et al., 1998; Phelps and LeDoux, 2005) and nonhuman mammals (Kapp et al., 1992; Fanselow and LeDoux, 1999; Davis and Whalen, 2001). It is also necessary for fear-motivated learning as lesions to the amygdala can disrupt the acquisition of active and passive avoidance conditioned responses in rats and rabbits (Roozendaal et al., 1993; Treit and Menard, 1997; Poremba and Gabriel, 1999; Lehmann et al., 2000). Furthermore, the amygdala plays a direct role in learning and memory as well as in modulating the acquisition and consolidation of memories that evoke emotional responses (McGaugh, 2000; Packard and Cahill, 2001).

\subsubsection{Amygdala anatomy and connections}

The amygdala is an almond-shaped subcortical structure that lies in the medial temporal lobe (for a visual representation of the amygdala's location, see Figure 1-3) and has a mean volume of approximately $1700 \mathrm{~mm}^{3}$ (David et al., 2002). The amygdala as a whole is comprised of approximately 13 nuclei and subnuclei that can be distinguished based on cytoarchitectonics, histochemistry, and connectivity (Krettek and Price, 1978; Pitkänen, 2000); all nuclei and subnuclei of the amygdala are extensively interconnected. Functionally, these nuclei are divided into three groups: the basolateral nuclear group (which is the largest and consists of the lateral, basal, and accessory basal nuclei), the centromedial group (including the central and medial nuclei), and the cortical nucleus (the smallest). The basolateral nucleus is considered the gateway to the amygdala as it receives most incoming sensory information, including highly processed visual inputs from the temporal cortex. Information then progresses on to the centromedial nuclei - the main source of output from the amygdala (Pitkänen, 1997).

The amygdala has many afferent and efferent connections encompassing a widespread number of both cortical and subcortical brain areas (Price et al., 1987; McDonald, 1996; 
Pitkänen, 2000). Inputs to the amygdala can arise from both cortical and thalamic structures, which supply sensory and memory information, as well as from hypothalamic and brain stem structures that send inputs from behavioral and autonomic systems. The cerebral cortex is the major source of sensory information to the amygdala from all sensory modalities. While information from olfactory, somatosensory, and gustatory comes in directly from primary sensory areas, the auditory and visual systems send information from their respective association areas. However, the amygdala can receive coarse visual information via the thalamus and superior colliculus, a topic that will be elaborated on further in Section 1.2.3. Several polymodal sensory areas also send information to the amygdala, including the prefrontal cortex and hippocampus.

The amygdala sends projections out to cortical, hypothalamic, and brain stem regions. Because the amygdala is involved in emotional responses that can be characterized by startle behavior, stress hormone release, sweating, and blood pressure and heart rate changes, it obviously has strong direct connections with regions of the brain stem controlling these behaviors (Sah et al., 2003; also see Figure 1-4) The amygdala does project to cortical sensory areas but these are not as robust as connections to the hypothalamus and brain stem. Within the amygdala there are also multiple connections between and within the individual nuclei. Tract tracing studies show that sensory information coming into the basolateral nuclei is processed locally and then progresses in a lateral to medial fashion to the centromedial nuclei, where information is then sent out to other parts of the brain (Krettek and Price, 1978; Pitkänen, 2000). Amygdala nuclei can also be grouped based on whether their principle output neurons are excitatory (glutamatergic) or inhibitory (GABAergic) (Swanson and Petrovich, 1998; Alheid, 2003; Sah et al., 2003). Major excitatory outputs arise from the basolateral complex as well as the cortical nuclei while inhibitory outputs originate from both the central and medial nuclei.

\subsubsection{The role of the amygdala in visual processing}

In the visual processing of faces, information can travel via two putative pathways: cortical and subcortical. In the cortical route, visual information from face stimuli travels from the lateral geniculate nucleus to the fusiform gyrus via primary visual cortex and then at some point in the processing stream is sent to the amygdala. This pathway is sensitive to facial detail due to its high spatial frequency visual information. In contrast, the subcortical route has a 
preference for coarse, low spatial frequency visual information and includes such structures as the superior colliculus, the pulvinar, and the amygdala (Johnson, 2005); the neocortex is circumvented (Figure 1-5). The processing of low spatial frequency information allows for the quick detection of stimuli, such as those that pose a danger to the individual. Vuilleumier et al. (2003) found that the amygdala responds to faces that are partially or entirely made of low spatial frequency components but not to faces made only of high spatial frequency information. Consistent with this study and the idea that the amygdala can receive information via a subcortical route are studies showing that the amygdala can process emotional stimuli, in particular fearful faces, without explicit attention or awareness (Anderson et al., 2003; Williams et al., 2004).

Visual processing undergoes modulation at all points in the stream due to massive feedback, and some feed-forward, loops. One way of viewing this is to see the initial sweep of feedforward social information as rapid and automatic, which is then followed by additional sequences of processing that can be modulated by top-down effects of controlled processing (Cunningham and Zelazo, 2007). At all levels, contextual information can modulate the processing of social information; for example, face information can be processed differently depending on its context (Adams and Kleck, 2003; Kim et al., 2004). The amygdala projects back to all levels of visual processing in a way that allows it to influence how visual information is processed by feeding back to areas from which it receives input while also feeding forward to regions it does not (Freese and Amaral, 2005). Fearful faces appear to facilitate early visual processing (Vuilleumier et al., 2004; Vuilleumier \& Driver, 2007), and there is evidence that the judgment of threat can be made even in backward-masking paradigms where faces are only shown for 39ms or less (Whalen et al., 1998; Whalen et al., 2004). Thus, an initial sweep of threat-related information may provide a 'gist' for the stimulus through the detection of basic features and therefore providing a foundation for further information processing.

\subsubsection{The amygdala and emotion}

Adolphs et al. (2002) argued that the amygdala is essential to linking the perception of a face to the retrieval of information about its social and emotional meaning. Even further, Adolphs has hypothesized that the amygdala is critical in the recognition of negative emotions from facial expressions. Negative emotions traditionally signal threat and danger; the correct 
recognition and identification of these emotions is imperative for avoiding perilous situations or encounters. Throughout many studies, the amygdala has shown the greatest response to facial stimuli that convey threat-related signals, such as fear and/or anger (Adolphs et al., 1994; Morris et al., 1996; Whalen et al., 1998), as well as in the processing of aversive and arousing stimuli (Calder et al., 2001; Adolphs, 2002; Phan et al., 2002; Phillips et al., 2003; Wager et al., 2003; Zald, 2003). However, the relationship between threat and the amygdala is not entirely reliable as activation to other emotions has been shown, indicating a more general involvement in stimulus salience and relevance. In addition to fear and anger, the amygdala has been shown to activate to facial displays of sadness and disgust; bilateral amygdala damage can cause deficits in the recognition of a wide range of negative emotions (Adolphs et al., 1999; Schmolck and Squire, 2001). The amygdala also responds to facial displays of happiness (Breiter et al., 1996; Yang et al., 2002), surprise (Kim et al., 2003), and neutrality (Iidaka et al., 2002) while some studies indicate that the amygdala actually responds equally to all emotional expressions (Yang et al., 2002; Winston et al., 2003).

The traditional view of the amygdala is that it is a key component in detecting stimuli that signal potential danger or threat in the immediate environment (Adolphs et al., 1999). More recent theories have generalized its function to the broader processing of distress signals (Blair et al., 1999), that it may indicate that potentially important information must be disambiguated (Whalen et al., 2001), or that it may operate as a "relevance detector" where it is involved in processing biologically relevant stimuli regardless of valence (Sander et al., 2003).

\subsubsection{The amygdala and fearful faces}

A fearful face has characteristic hallmarks in the way the eyes widen, the eye whites enlarge, and the mouth pulls back into a grimace. The eye region itself appears to be especially useful in discriminating fear from other emotional expressions (Smith et al., 2005). Patients with amygdala lesions have been reported to show greater deficits in the recognition of fearful faces relative to other emotions, which is suggested to occur because patients are unable to use the information displayed by the eyes (Adolphs et al., 1994). Furthermore, fearful eyes shown in isolation (devoid of other face parts and features) and without context can elicit amygdala activation (Morris et al., 2002; Whalen et al., 2004), underscoring the importance of the eyes in the detection of fear. Whalen and colleagues reported a greater amygdala response to masked 
fearful eye whites (the sclera of the eye) than to masked happy eye whites and proposed that this difference in amygdala response was specifically due to the scleral field increases representative of fearful faces. Recognizing such a simple signal would allow the amygdala to quickly identify fear and rapidly respond to a threat. In addition, the eyebrows of the fearful face are characteristic in the way that they are furrowed yet also raised; it is possible that this cue along with the spatial relationship between the eyes and eyebrows are also important in detecting fearful expressions (Leppänen et al., 2008).

The role that the mouth plays in the detection of fearful facial expressions is not clear. While normal observers tend to use the eye region to detect fear, the mouth may be used to detect other emotions such as surprise (Smith et al., 2005) - an expression that also elicits an increase in visible whiteness. It has been reported that fear recognition deficits in individuals with autism may be associated with their tendency to fixate on the mouth rather than looking at the eyes (Spezio et al., 2006). In addition, patients with lesions to the amygdala who have difficulties recognizing fearful faces tend to spend more time looking at the mouth rather than the eyes (Adolphs et al., 2005). These findings suggest that the fearful mouth may not impart enough information for proper emotion identification. Morris et al. (2002) did examine the effects of a fearful mouth in the context of a neutral face but did not explicitly report if this produced a response in the amygdala. So while the mouth may not be used to recognize fear, it is unknown how it can be used to discriminate fear from other emotions such as surprise. 


\subsection{Amygdala Dysfunction}

In Section 1.2.1, classic studies that helped to understand amygdala function were presented; in particular, the relationship between amygdala lesions and behavioral deficits were key in defining the role of the amygdala in social and emotional processing. More recently, the amygdala has been shown to play a large role in many psychological and social disorders. Additionally, there is a large body of evidence implicating amygdala dysfunction in contributing to autism; this section will briefly address some psychological and social disorders thought to involve the amygdala.

\subsubsection{Amygdala involvement in psychological and social disorders}

A number of disorders are thought to involve the amygdala, which is perhaps not surprising considering its involvement in emotional and social processing. In borderline personality disorder (BPD), one common behavioral characteristic is an intense and rapidly changing mood state. Herpertz et al. (2001) found elevated BOLD responses in the amygdala bilaterally when viewing emotionally aversive pictures, thought to reflect the intense and slowly waning emotions that are often observed in BPD patients to low-level stressors. Other studies have found a weak correlation between the amygdala and prefrontal cortex activations (New et al., 2007), and decreased amygdalar and hippocampal volumes in those with BPD (Nunes et al., 2009). Schizophrenia, distinguished by abnormalities in the perception or expression of reality, also displays decreased amygdala and hippocampal volumes, along with other limbic structures, that may attenuate the response to emotional stimuli (Lawrie et al., 2003). Individuals with schizophrenia often show difficulties in interpreting social information, such as faces, and this may involve a hyper-activation of the amygdala (Marwick and Hall, 2008), as negative face discrimination tasks elicited exaggerated amygdala activation when schizophrenic patients judged emotional intensities (Kosaka et al., 2002). However, much of the data on the amygdala and schizophrenia is varied and thus it has been difficult for researchers to make clear conclusions.

Both the amygdala and insula are thought to be primarily involved in the fear response that is apparent in many anxiety disorders. In social anxiety disorder, in which individuals experience a considerable amount of stress and an inability to function in social situations, 
hyperactivation of both the insula and the amygdala was observed (Etkin and Wager, 2007). This was also true for patients suffering from post-traumatic stress disorder (PTSD) and specific phobia. Patients with PTSD experience hypervigilance, an enhanced state of anxiety and with increased levels of arousal, a high responsiveness to stimuli, and constant surveillance for threat. One model of PTSD contends that the exaggerated response of the amygdala occurs in combination with a lack of top-down control by the medial prefrontal cortex (Liberzon and Sripada, 2008). In general, it appears that PTSD, social anxiety disorder, and specific phobia reflect a general state of heightened activity in fear circuits, which is why these disorders share a number of the same symptoms.

The amygdala dysfunction is suspect in many disorders, such as the ones outlined above. Because the amygdala has extensive connections with many brain regions, it is in a prime position for its activity to modulate or be modulated by these areas, explaining why the amygdala often shares similarities in dysfunction with other brain areas, such as the insula, hippocampus, and orbitofrontal cortex.

\subsubsection{Autism Spectrum Disorder (ASD)}

Autism is a brain development disorder that is typified by impairments in social interaction and communication that begins early in childhood; individuals with autism often exhibit face processing deficits and some of this is attributed to the amygdala dysfunction. Although it is highly variable, it leaves children unable to form normal methods for communication and relationships. Children with autism often isolate themselves, shun contact, and are frequently obsessive and repetitive in their interests. In the book titled "Autism: the facts", Baron-Cohen and Bolton (1993) describe some of the behaviors representative of autism: "Children with autism may behave in strange ways. They may look past you, or only very briefly at you, and make you feel as if you are an unimportant part of their world".

Individuals with ASD display a range of social abnormalities; at the highest-functioning end of the scale are those individuals with Asperger's syndrome, who rank more mildly with respect to their social impairments. The most common include 1) withdrawal, 2) a lack of attention to other people, 3) a lack of eye contact, 4) an unawareness of the feelings of others, 5) an inability to understand and ascribe to social norms, and 6) treating people as inanimate objects; all depict deficits in social interest and understanding (Baron-Cohen and Bolton, 1993). 
Individuals with autism also display deficits with respect to face processing tasks, including visual scanning, memory for faces, and affect recognition (Marcus and Nelson, 2001; Grelotti et al., 2002). They do not appear to perceive faces holistically, yet rely on features (Joseph and Tanaka, 2002) and often characterize faces based on a single salient feature (Schwarzer, 2000).

Individuals with autism do not seem to understand how to communicate non-verbally with their eyes, nor can they read emotions and expressions on the faces of others. Research shows that they do not fixate normally on faces in videos and photographs (Klin et al., 2002, Pelphrey et al., 2002), and often gaze at the mouth in social situations (Grandin, 1996). Furthermore, it appears that there is actually an active aversion to fixating on the eyes (Richer and Cross, 1976; Spezio et al., 2007) which is thought to explain abnormally higher fixations on the mouth (Figure 1-6). When explicitly directed to attend to the eyes, ASD individuals can discriminate gaze direction and identify whether someone is looking at them or not, however they appear to use low-level information - such as pupil direction - rather than using detailed information from the eyes for social cues (Ristic et al., 2005).

fMRI studies indicate individuals with ASD are impeded in face processing and this has been documented via weak activation to faces in the fusiform gyrus (Critchley et al., 2000; Pierce et al., 2001; Hubl et al., 2003; Grelotti et al., 2005), as well as activation of other brain regions not typically associated with faces (Schultz et al., 2000; Hubl et al., 2003; Grelotti et al., 2005; Schultz et al., 2003). In addition, regions associated with social processing, including the STS (Pelphrey et al., 2004) and the amygdala, are also impaired in individuals with ASD.

Amygdala dysfunction is thought to underlie the abnormal patterns of face fixation and the lack of gazing at the eyes of another (Baron-Cohen et al., 2000). Activation of the amygdala strongly correlates with the time that ASD individuals spend fixating the eyes (Dalton et al., 2005), further supporting the notion of eye avoidance. Additionally, the amygdala is critical for emotion processing yet autistic individuals do not appear to use the amygdala when viewing eye stimuli and instead place a greater processing load on the temporal lobes (Baron-Cohen, 2000). Therefore, understanding how the normal amygdala uses information from the eyes and mouth may aid in understanding the face processing deficits seen in autistic individuals and lead to better strategies for helping them overcome these shortcomings. The experiments presented subsequently (Chapters 4, 5, 6) attempt to further understand how and if the amygdala can use information from face parts to recognize threatening emotions from non-threatening ones; the 
results of these experiments could lend more evidence to the growing body of literature that links amygdala function to deficits seen in autistic individuals. 


\subsection{Conclusions}

This section outlined how facial expressions are crucial for human nonverbal communication and how one particular structure in the brain, the amygdala, is involved in detecting fear in another's face. As amygdala dysfunction is thought to underlie a number of psychological and social disorders, determining how information from the face is extracted may aid in understanding the amygdala's involvement in such disorders. The information presented here provides a basis for understanding the rationale and objectives behind the experiments outlined in Chapters 3, 4, 5, and 6. 


\subsection{Figures}

Figure 1-1 (1)

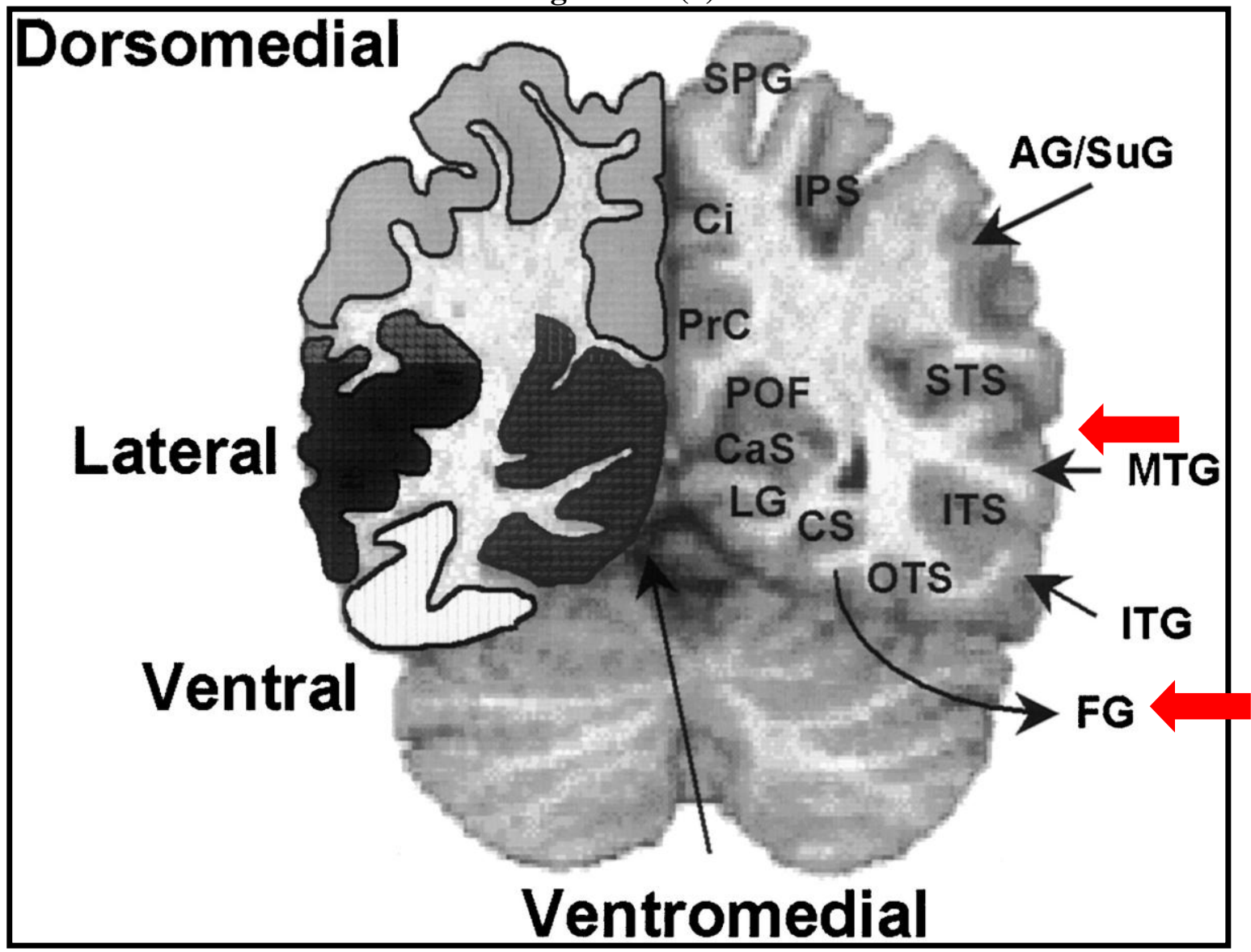

Figure 1-1. Coronal anatomical image of a human brain showing location of certain structures, including the STS and FG (red arrows). STS: superior temporal sulcus; MTG, middle temporal gyrus; ITS, inferior temporal sulcus; ITG, inferior temporal gyrus; OTS, occipitotemporal sulcus; FG, fusiform gyrus; CS, collateral sulcus; LG, lingual gyrus; CaS, calcarine sulcus; $\mathrm{POF}$, parieto-occipital fissure; $\mathrm{PrC}$, precuneus; $\mathrm{Ci}$, cingulate gyrus and sulcus; SPG, superior parietal gyrus; IPS, intraparietal sulcus; $\mathrm{AG} / \mathrm{SuG}$, angular or supramarginal gyri. This illustration is borrowed from another source (Puce et al., 1998). 
Figure 1-2 (2)

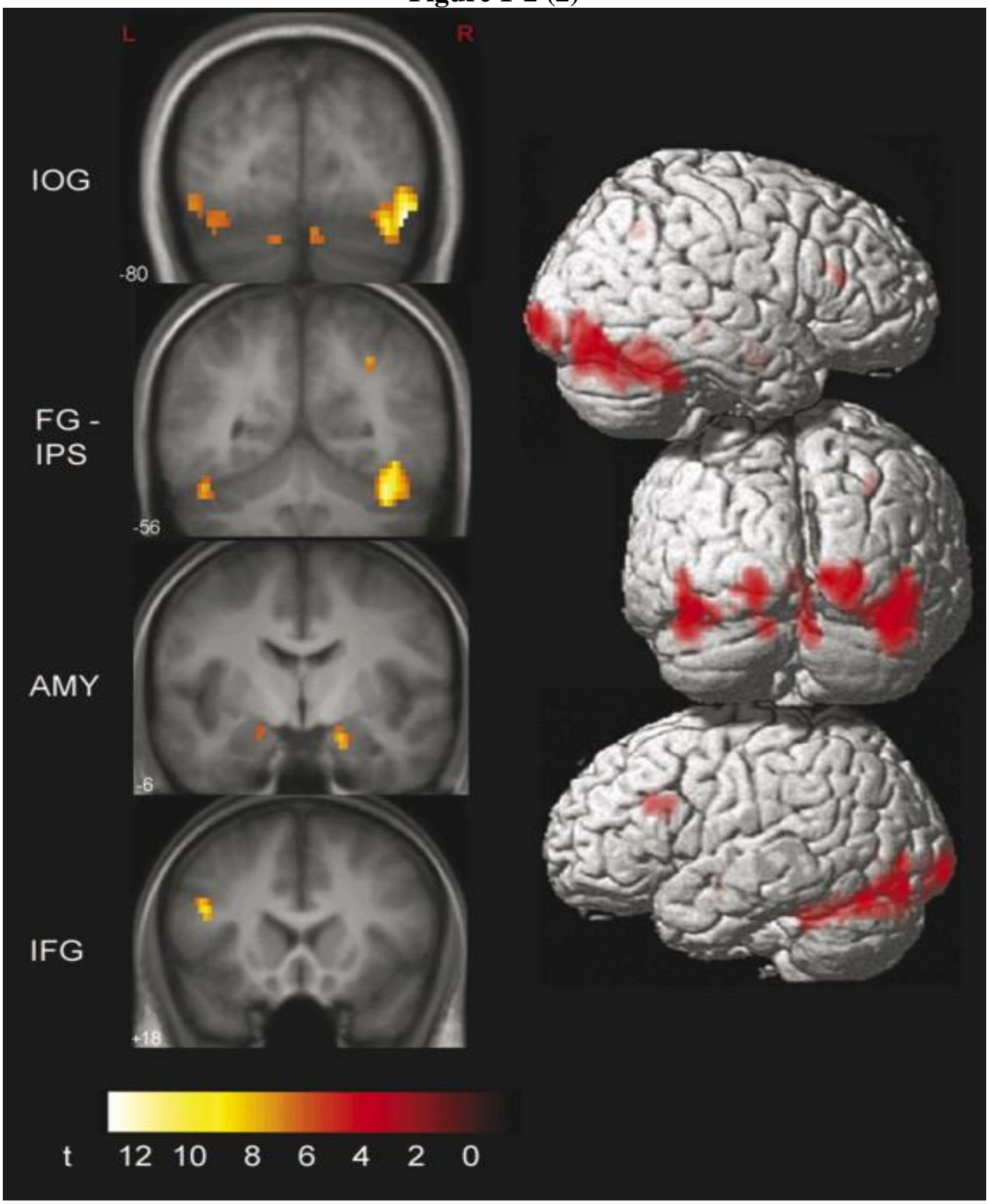

Figure 1-2. Areas of significant activation for whole faces compared to masks, illustrating regions of the brain involved in face processing. IOG $=$ inferior occipital gyrus. $\mathrm{FG}=$ fusiform gyrus. IPS = intraparietal sulcus. AMY = amygdala. IFG = inferior frontal gyrus. Figure and caption borrowed from another source (Benuzzi et al., 2007). 
Figure 1-3 (3)

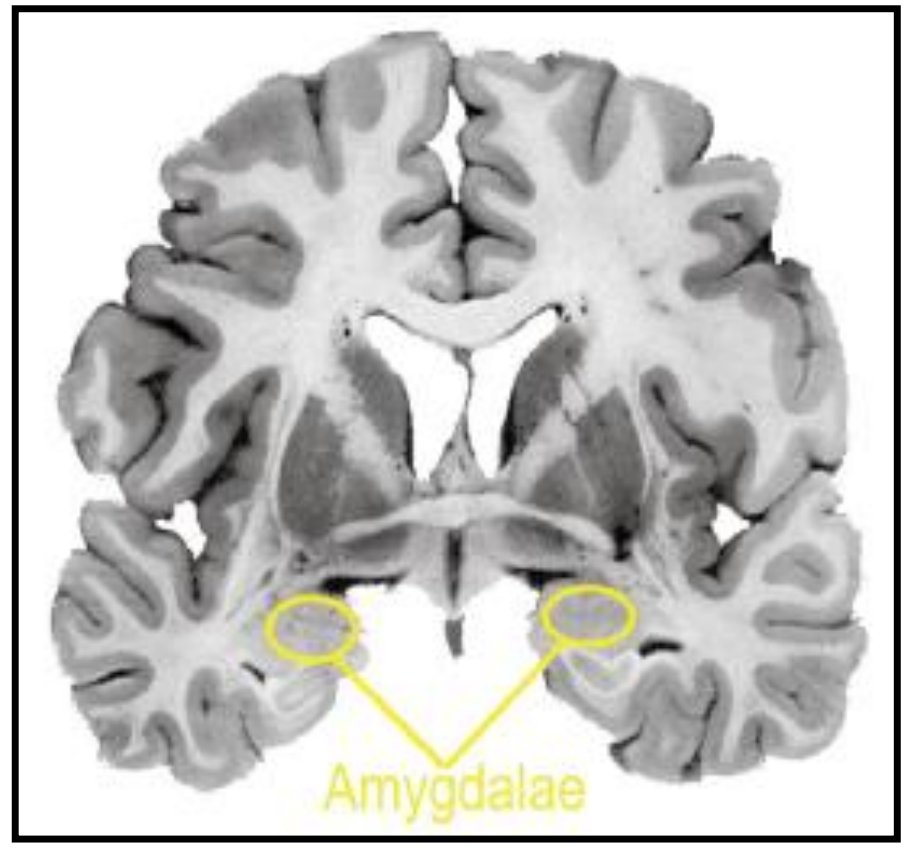

Figure 1-3. Human amygdalae. Coronal section of the human brain indicating the location of the amygdalae (yellow) deep within the temporal lobes. Figure and caption were borrowed from another source (Davidson and Irwin, 1999). 
Figure 1-4 (4)

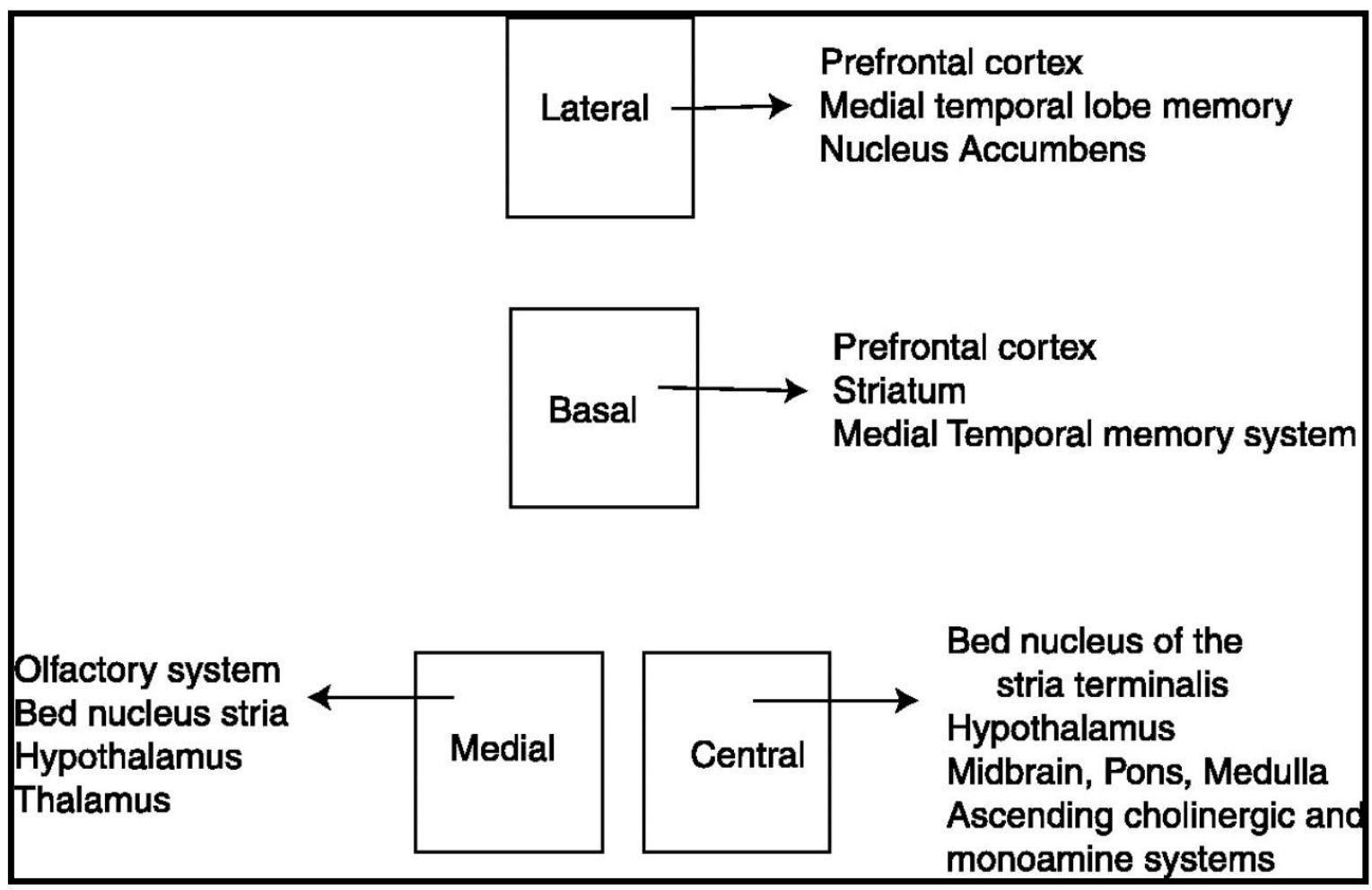

Figure 1-4. Depiction of outputs from lateral, basal, central, and medial nuclei of the amygdala. This illustration was borrowed from another source (Sah et al., 2003). 
Figure 1-5 (5)

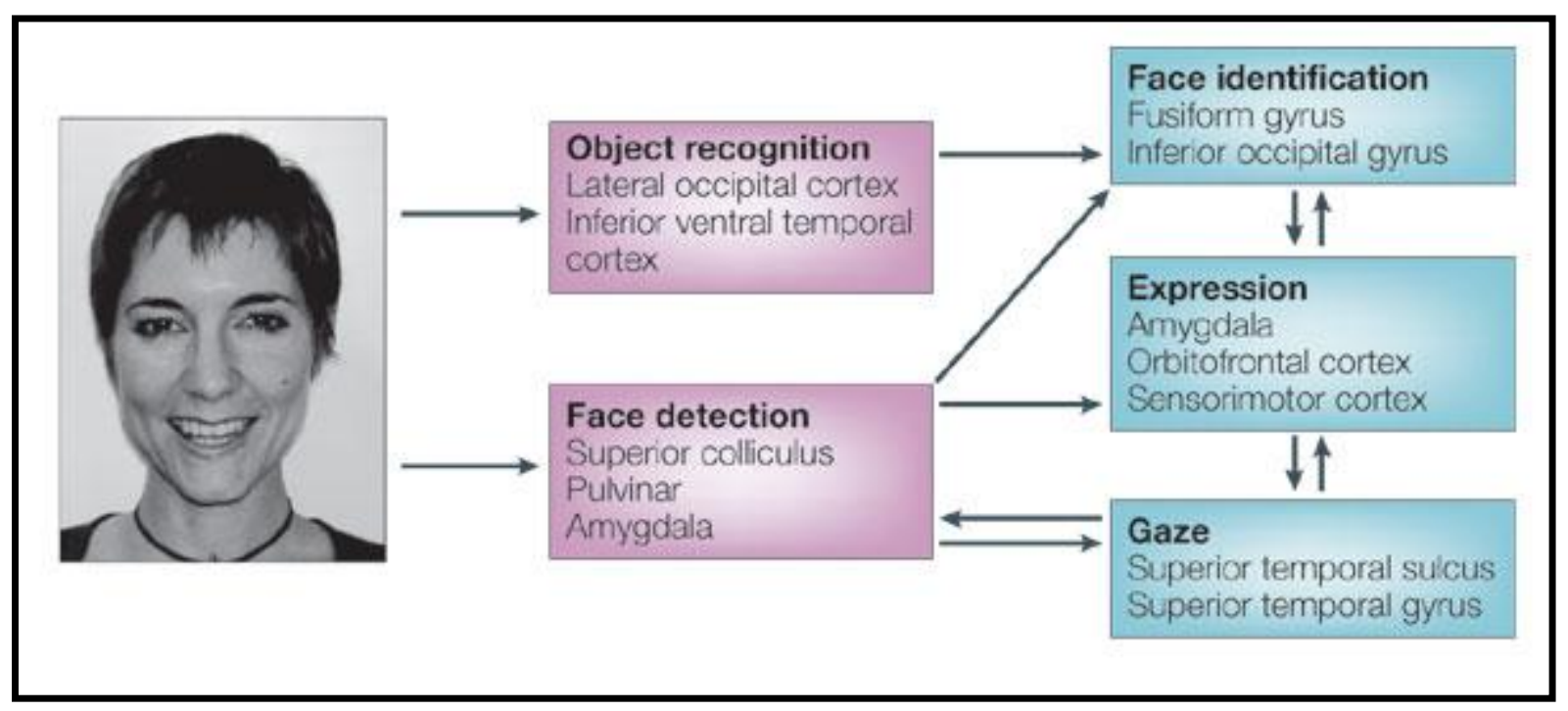

Figure 1-5. The dual route model of face processing in adults. The model illustrates how faces can be processed through both a subcortical face-detection route involving the superior colliculus, pulvinar, and amygdala and also a cortical route. The subcortical route modulates processing in structures that are fed by the cortical pathway and are involved in face identification (fusiform gyrus and inferior occipital gyrus), facial expression (amygdala, orbitofrontal cortex, sensorimotor cortex) and eye gaze (superior temporal sulcus). This illustration and caption were borrowed from another source (Johnson, 2005). 
Figure 1-6 (6)

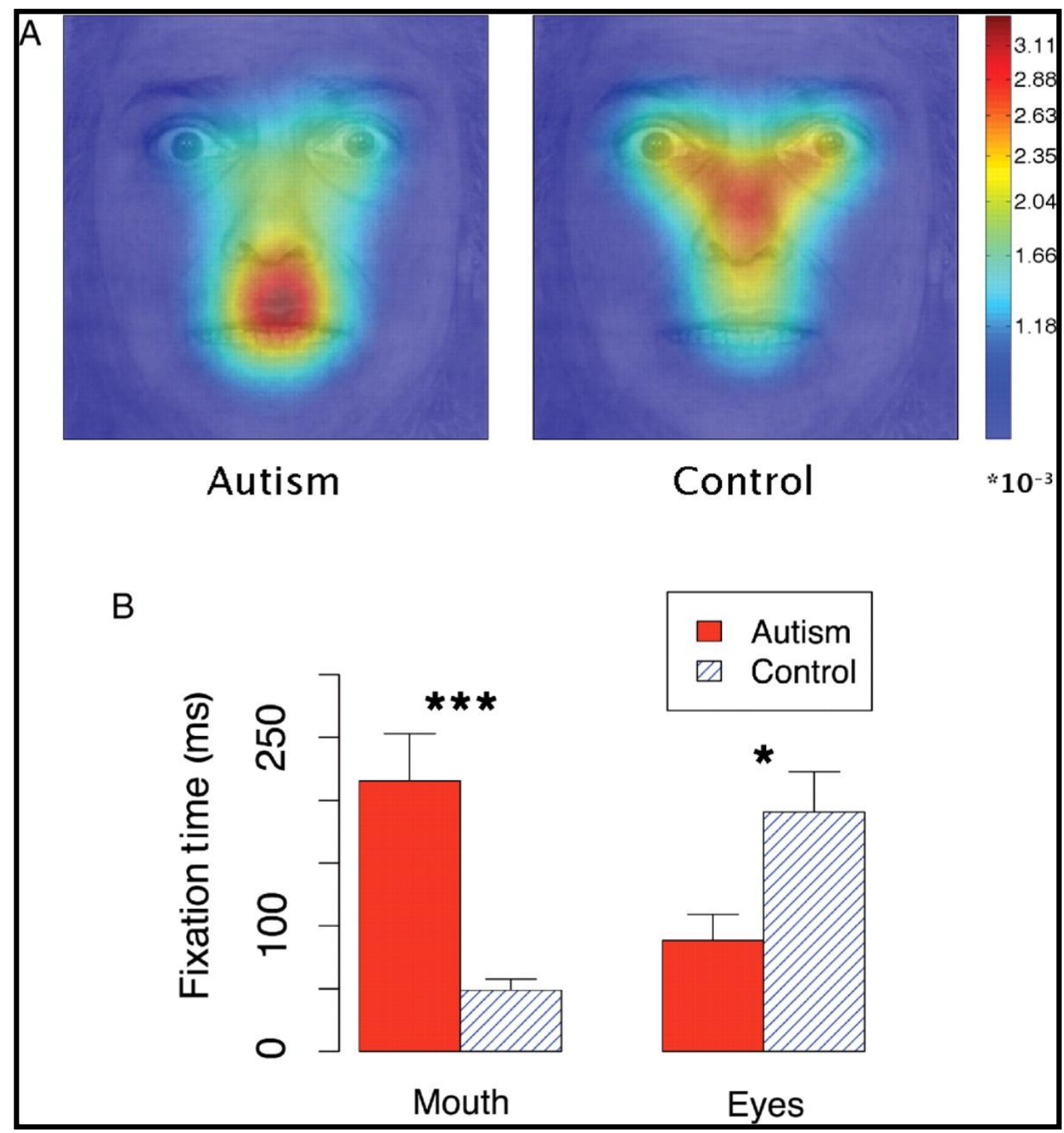

Figure 1.6 Fixations made during the first $1000 \mathrm{~ms}$ of a face presentation. A. Fixation density maps showing how the autism group spent more time looking at the mouth region of the face while controls fixated on the eye region more than the autistic group and fixated the mouth less. B. Average time spent viewing the mouth and the eyes. Illustration was borrowed from another source (Neumann et al., 2006). 
CHAPTER 2: Objectives and Rationale 
The amygdala is known to play an essential role in detecting fear in the facial expressions of others, yet little is known about how facial information is used by the amygdala to enable the identification of fear. Research indicates that fearful facial expressions are strong, salient stimuli that can increase neural responses in the networks dedicated to processing emotion. Determining the role that facial cues play in the identification of fear will help to better understand the underlying nature of social and emotional deficits present in individuals with autism and Asperger's syndrome as amygdala dysfunction appears to play a role in such disorders. The experiments in this study were constructed to meet two separate objectives via three individual experiments using functional magnetic resonance imaging (fMRI). This current chapter presents each objective in addition to the rationale behind them.

\subsection{Objective 1 - Characterize the role of the eye region in the neural recognition of fear}

Previous studies have determined the importance of the eye region when identifying certain emotions - mainly fear - in the face of another as well as the importance of the amygdala for directing attention to this area of the face. In particular, the increase in eye white area (EWA) characteristic of a fearful face shown in isolation is enough information to alert the amygdala to the presence of a fearful face. Accordingly, it is thought that the amygdala uses the "simple rule" of increased EWA to prime other cortical areas so as to ready for an appropriate behavioral response. However, it us unknown whether the amygdala is able to discriminate fear from other conditions that elicit similar increases in EWA.

Chapter 3 (Experiment 1) presents preliminary data that led to the formation of the experiments outlined in Chapters 4 and 5. Experiments 2 and 3 (Chapters 4 and 5, respectively) seek to determine if the amygdala is able to distinguish between conditions with similar (shift in gaze) or identical (surprise) increases in EWA to that seen in fear; when presented with comparable EWA changes, does the amygdala still select fear? Changes in gaze direction, as well as expressions of surprise, increase EWA relative to that observed in a neutral expression; both are also important social and emotional indicators that something is happening outside of the viewer's immediate vision. In individuals with autism, there is often a lower than normal BOLD (blood oxygen level dependent) response to fearful faces in the amygdala and the fusiform gyrus; this is thought to stem from a failure to look at the eye region of the face. Thus, 
revealing which facial cues best evoke activity in the neural systems that underlie fear recognition may then assist in improving the recognition of facial affect in individuals with social cognitive disorders.

In Objective 1, I hypothesize that the amygdala will be able to discriminate fear from other expressions that elicit similar (Experiment 2) or identical (Experiment 3) increases in EWA. I will test this hypothesis using stimuli that depict only the eye (Experiment 2) or the eye/eyebrow region (Experiment 3) of the face and will then compare the fMRI BOLD responses that result from viewing these stimuli.

\subsection{Objective 2 - Characterize the role of the mouth region in the neural recognition of fear}

Little is known about what role the mouth region plays when discriminating fear from other emotions. It may be that the mouth does play a role in fear detection, but could be less effective than the eye region for the simple reason that subjects more often focus on the eyes of another when attending to emotion. If patients with amygdala damage and those with certain social cognitive disorders are unable to use information from the eyes when viewing facial expressions of fear and focus instead on the mouth, is the amygdala able to discriminate a fearful mouth from other expressions? Additionally, how do the fusiform gyrus and STS, two brain areas previously implicated in the processing of faces, face parts, and biological motion, respond to a fearful mouth compared to that of other emotions? Experiment 4 (Chapter 6) is designed to address these questions by using stimuli composed only of the mouth region that convey either fearful, surprised, happy, or neutral expressions to see if each will elicit differential patterns of activation in the brain.

In Objective 2, I hypothesize that the amygdala will not be able to discriminate fear from other emotional expressions using only the mouth region of the face (Experiment 4). I will test this hypothesis using stimuli that depict only the mouth and will then compare the fMRI BOLD responses that result from viewing these stimuli. 


\section{CHAPTER 3: Experiment 1 - Preliminary Experimental Data: Masking Eye Gaze Changes}




\subsection{Introduction and Rationale}

The eyes can convey a wealth of information and, along with faces and facial expressions, are essential to human social interactions. When communicating with others, we follow any breaks in eye contact to determine the direction of another's attention and to what they are attending. We can learn about things in our environment by following another's line of sight and monitoring their reactions and emotional expressions. Incoming social information such as this greatly modifies our own behaviors, allowing us to act appropriately in a variety of social situations. Additionally, it has been shown that activity levels in brain regions involved in processing faces, like the fusiform gyrus, the superior temporal sulcus, and the intraparietal sulcus, can be influenced by gaze direction and changes in gaze (George et al., 2001; Pelphrey et al., 2003).

Detecting the presence of fear in another's face and/or a shift in gaze direction is important, from an evolutionary point of view, in order to avoid threats in the immediate environment. Given that eyes are a crucial part of the face for human interactions, can the brain detect changes in gaze direction even when stimuli are backwardly masked and presented subliminally in the same manner that the amygdala can detect backwardly masked fear (Whalen et al., 2004)? It is hypothesized that shifts in eye gaze would be detected by the brain - especially the STS and possibly amygdala - even when presented at levels thought to be below conscious detection (<40msec; Whalen et al., 1998) so that subjects claim to not even be aware of seeing them. If subjects are unable to report seeing shifts in eye gaze at this same rapid presentation time, then fMRI could be used to compare the brain responses to both fear and gaze shift stimuli to see if the brain processes these expressions differently. Is a shift in gaze as biologically relevant as fearful eyes in that it can be detected by the brain although subjects do not? However, behavioral data presented here revealed that subjects were able to detect and identify putative 'subliminal' shifts in gaze above chance levels, and therefore brain imaging data was not acquired due to the fact that this finding refuted the criteria that were chosen. Although fMRI data was not collected in this experiment, the behavioral results were informative enough to conclude that shifts in eye gaze are extremely noticeable and exceptionally salient, even when presented very briefly. These results also helped formulate the experimental methods for Experiment 2 (Chapter 4 ) in that stimulus presentation times were above the level of conscious subject detection and there were no perceptual disparities between fear and shifts in eye gaze. 


\subsection{Methods}

\subsubsection{Participants}

Twelve neurologically normal subjects ( 7 female, all right-handed, aged 23-50 years of age) consented to participate in a study approved by the Institutional Review Board of West Virginia University. All subjects had either normal or corrected-to-normal vision.

\subsubsection{Stimuli}

Stimuli were selected from the JACFEE/JACNeuF series of faces by Ekman and Matsumoto (Paul Ekman Group LLC, Berkeley, CA, USA) and could belong to one of three categories: fear, gaze, and neutral. All images were altered in Adobe Photoshop 7.0 with an initial conversion to grayscale and then a uniform cropping to leave a $118^{\circ}$ by $38^{\circ}$ rectangle centered on the eyes. All categories used 7 different identities and were equally balanced with respect to gender and race. Gazeshifted stimuli were created from neutral faces that were altered to look either to the left or the right of the observer. Mean luminance and contrast were equated for all stimuli. From these stimuli, 5 conditions were created: subliminal fear, subliminal eye gaze, liminal fear, liminal gaze, and control (neutral expression).

\subsubsection{Task design}

The task consisted of an event-related design spanning 4 runs. Each run contained 49 randomly-presented conditions, and only one of the 5 conditions was shown per trial (Figure 31). Each trial began with a fixation cross presented centrally for $500 \mathrm{~ms}$ (millisecond). At the offset of the cross, a train of eye stimuli were presented for 600ms: neutral eyes matching the identity of the condition would appear at $100 \mathrm{~ms}$ each and were then followed by one of the conditions, with the neutral eyes appearing again at $100 \mathrm{~ms}$ each. To vary where the condition appeared in this stream of neutral eyes, a condition could be preceded by 2, 3 or 4 neutral eyes; the number of neutral eyes appearing before the condition determined how many neutral eyes would follow the condition as all trials lasted $600 \mathrm{~ms}$. The transition between all eye stimuli gave the appearance of each picture flashing on and off. Trials were separated by an intertrial interval consisting of a black screen for 3 seconds. Each condition was shown 42 times across all 4 runs. Stimuli were delivered using Presentation software (Version 9.90, Neurobehavioral Systems, 
Albany, CA, USA). For liminal conditions (liminal fear, liminal gaze, and control conditions), stimuli appeared for $100 \mathrm{~ms}$ in the stream of neutral eyes. For subliminal conditions, either the fearful or gaze-shifted eyes would appear for $30 \mathrm{~ms}$ and would then seamlessly be followed by the presentation of neutral eyes for $70 \mathrm{~ms}$, for a total of $100 \mathrm{~ms}$ within the stream of neutral eyes (Figure 3-1). At the end of each trial, a response screen would prompt subjects to make a response via button press.

Subjects were told that they would see images of eyes appearing in the center of the screen and that they were to maintain fixation at this central location for the duration of each run. Using a button box, they were to respond whether they detected a fearful face, a shift in eye gaze, or a neutral expression. 


\subsection{Results}

The average percent correct response and response time for all 12 subjects per condition type are listed in Table 3-1.

\begin{tabular}{|l|c|c|}
\hline Table 3-1 & $\begin{array}{c}\text { Average Percent Correct } \\
(\mathbf{n = 1 2})\end{array}$ & $\begin{array}{c}\text { Average Correct } \\
\text { Response Time(ms) }\end{array}$ \\
\hline Liminal Eye & $79.9 \pm 14.4 \%$ & $709.4 \pm 82.6$ \\
\hline Liminal Fear & $86.7 \pm 13.0 \%$ & $705.1 \pm 85.3$ \\
\hline Subliminal Eye & $\mathbf{4 6 . 6} \pm \mathbf{2 7 . 4 \%}$ & $769.1 \pm 153.5$ \\
\hline Subliminal Fear & $15.0 \pm 21.5 \%$ & $755.2 \pm 185.9$ \\
\hline No Expression & $77.7 \pm 11.1 \%$ & $801.4 \pm 90.5$ \\
\hline
\end{tabular}

The formula for calculating percent chance response is $(1 / n)$ where $n=$ number of alternatives. In this experiment there were 3 choices: gaze shift, fear, or neutral expression thus the percent chance of getting an answer correct is $33.3 \%$. Although the subliminal fear condition was below chance $(15.0 \%)$, the subliminal eye condition was not (46.4\%) indicating that subjects could detect the presence of a shift in gaze in these trials despite the fact that it was backwardly masked. It was expected that the liminal eye, subliminal eye, and neutral expression conditions would have percent accuracies above chance levels.

A series of single-factor ANOVAs was run between conditions to determine if percent accuracy varied as a function of condition (Table 3-2).

\begin{tabular}{|l|c|}
\hline Table 3-2 & $p$-value \\
\hline Liminal Eye vs. Liminal Fear & $\mathrm{p}<0.007 *$ \\
\hline Subliminal Eye vs. Subliminal Fear & $\mathrm{p}<2.15 \times 10^{-8} *$ \\
\hline Liminal Eye vs. Subliminal Eye & $\mathrm{p}<3.02 \times 10^{-10} *$ \\
\hline Liminal Fear vs. Subliminal Fear & $\mathrm{p}<9.47 \times 10^{-34} *$ \\
\hline
\end{tabular}

* indicates that value is statistically significant 
A single-factor ANOVA was run between conditions to determine if the average response times varied as a function of condition (Table 3-3).

\begin{tabular}{|l|c|}
\hline Table 3-3 & $p$-value \\
\hline Liminal Eye vs. Subliminal Eye & $\mathrm{p}=0.80$ \\
\hline Subliminal Eye vs. Subliminal Fear & $\mathrm{p}=0.73$ \\
\hline Liminal Eye vs. Subliminal Eye & $\mathbf{p}<\mathbf{0 . 0 2 *}$ \\
\hline Liminal Fear vs. Subliminal Fear & $\mathrm{p}=0.11$ \\
\hline
\end{tabular}

* indicates that value is statistically significant 


\subsection{Discussion}

Previous studies have shown that backward masking can "hide" the presentation of emotional faces so that the subject is not aware that an emotional face has been shown (Esteves and Öhman, 1993; Rolls and Tovee, 1994; Whalen et al., 1998). Specifically, Esteves and Öhman (1993) showed that a time interval of <40ms between the onset of the target and the mask that follows it is the most successful in obscuring a target. Given this data, it was surprising that human subjects in our current study performed better than chance when it came to detecting the presence of gaze shifts in our subliminal eye condition (see Section 3.3). However, it is important to note that the studies referenced above used a variety of emotional faces, such as happy, angry, and fearful, but did not specifically look at shifts in gaze direction where eye movements may be a more salient feature.

Whalen et al. (1998) demonstrated that the amygdala can detect the presence of fearful eyes even when the subject is not aware of seeing this stimulus. This is hypothesized to result from the increase in eye white area that is a hallmark characteristic of the fearful face; it is both salient and powerful as it can be seen nearby or far away and does not rely on the presence of facial details. Similar to the fearful eye is a shift in gaze as the sclera becomes more prominent and noticeable when the iris is shifted toward the outer edges of the eye. Human eyes are wider in the horizontal direction and thus expose a much higher proportion of sclera than other primates (Kobayashi and Koshima, 1997). This would readily explain why it is difficult to mask shifts in gaze; fearful eyes result from a general widening of the eye while the iris remains central, producing an overall increase in whiteness for the entire eye. Gaze shifts, especially to the left or right, result in a large white space generated from the iris changing its position to the far side of the eye perimeter and the change may be more noticeable to the viewer. Thus, gaze shifts can also act as salient and powerful stimuli in a manner similar to fearful eyes but may be more detectable due to the fact that they can convey a variety of social and emotional signals.

Moreover, many studies have shown that the eyes are the most attended region of the face when performing social cognitive tasks. The eyes are extremely effective at triggering the attention of human infants (Gilga and Csibra, 2007), and humans in general spend more time gazing at the internal features of the face - such as eyes, nose, mouth - rather than the external features of the face such as hair and face shape (Yarbus, 1967). The eye region itself is the most attended portion of the face regardless of what task is being performed (Adolphs, 2008) and face 
detection and perception are both impaired when the eye region is occluded. With respect to gaze in particular, it acts as an important social cue to indicate the focus of another's attention as well as to cue attention to places and objects within the environment. Target detection is faster when a centrally presented face gazes at the side of the display where the target will appear than when it is directed in the opposite direction of the target (for a review, see Frischen et al., 2007).

Therefore, this may reflect an automatic and bottom-up driven orienting mechanism of attention that is difficult to repress (Driver et al., 1999; Langton and Bruce, 1999; Ricciardelli et al., 2002). Thus, the combination of increased visual salience and role as a social cue lends much support to our findings that shifts in eye gaze are difficult to mask from subject awareness.

Future studies could pair shifts in gaze with emotional expressions to examine whether certain emotions are faster to detect when paired with gaze movements, or if altering the time of stimulus presentation to below $10 \mathrm{msec}$ can conceal their appearance - something our software was not equipped to undertake at the time. In conclusion, this behavioral experiment revealed that unlike fearful faces and fearful eyes, shifts in gaze cannot be backwardly masked so that viewers are unable to report their appearance. Based on these results, the following experiment (Chapter 4) comparing fearful eyes and eyes that shift in gaze used stimulus presentation times well above perceptual thresholds for detection. 


\subsection{Figures}

Figure 3-1 (7)

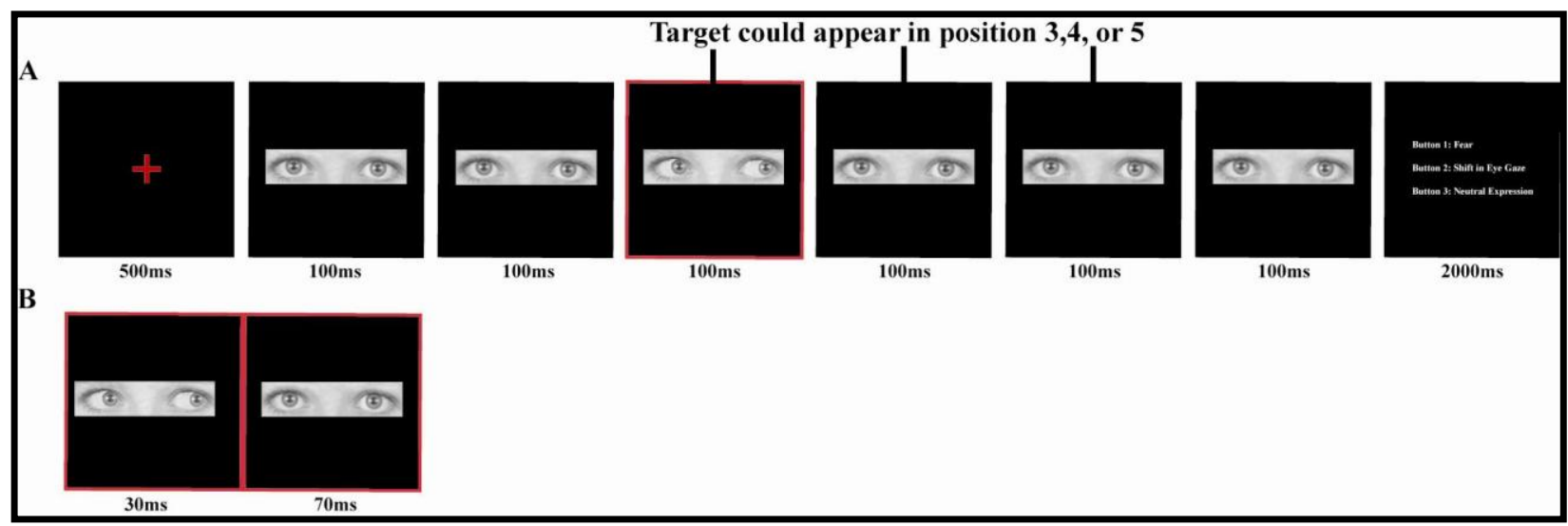

Figure 3-1. Example of single event trial. A. Subjects viewed one of four conditions, in this case a shift in eye gaze (outlined in red), between sets of neutral eyes. The condition, or "target", could be preceded by 2, 3, or 4 neutral sets of eyes and would be followed by either 1,2 , or 3 sets of neutral eyes so that the entire train of eye stimuli was shown for $600 \mathrm{~ms}$. The train of eye stimuli was then followed by a response prompt. The target in this condition is a liminal stimulus as it is shown for $100 \mathrm{~ms}$. B. Example of a subliminal stimulus presentation. Instead of a single target image appearing for $100 \mathrm{~ms}$, as seen in A, the target would be shown for $30 \mathrm{~ms}$ and then would immediately be followed by a neutral eye image, effectively masking the presentation of the target. The transition between these two images was seamless so that subjects could not detect the presence of a target simply through motion changes. 


\section{CHAPTER 4: Experiment 2 - The Left Amygdala Knows Fear: Laterality in the Amygdala Response to Fearful Eyes}

This chapter was published in Social Cognitive and Affective Neuroscience, March 2008, 3(1); 47-54. It has been modified from its original format. 


\subsection{Abstract}

The detection of threat is a role that the amygdala plays well, evidenced by its increased response to fearful faces in human neuroimaging studies. A critical element of the fearful face is an increase in EWA, hypothesized to be a significant cue in activating the amygdala. However, another important social signal that can increase EWA is a lateral shift in gaze direction, which also serves to orient attention to potential threats. It is unknown how the amygdala differentiates between these increases in EWA and those that are specifically associated with fear. Using functional magnetic resonance imaging, we show that the left amygdala distinguished between fearful eyes and gaze shifts despite similar EWA increases whereas the right amygdala was less discriminatory. Additional analyses also revealed selective hemispheric response patterns in the left fusiform gyrus. Our data show clear hemispheric differences in EWA-based fear activation, suggesting the existence of parallel mechanisms that code for emotional face information. 


\subsection{Introduction}

In humans, the ability to recognize facial expressions is critical for the transmission of emotional and social information (Adolphs, 1999). Detecting emotion-especially fear-from another's face can aid in identifying potential threats and allow for a rapid formulation of appropriate behavioral responses. Previous neuroimaging studies have demonstrated that fearful faces strongly and reproducibly activate the amygdala, even when subjects are not aware a fearful face was presented (Whalen et al., 1998; Morris et al., 1999). Additionally, patients with lesions to the amygdala have been reported to show greater deficits in the recognition of fearful faces relative to other emotions (Adolphs et al., 1994). These deficits have been suggested to occur because patients are unable to use the information displayed by the eyes, as guiding them to look at the eye region leads to a temporary increase in the recognition of fearful expressions (Adolphs et al., 2005). In healthy individuals, information from the eye area appears to be especially useful in discriminating fear from other emotional expressions (Smith et al., 2005). Furthermore, fearful eyes shown in isolation and without context elicit fMRI activation in the amygdala (Morris et al., 2002; Whalen et al., 2004), further underscoring the importance of the eyes in the detection of fear.

What makes fearful eyes so salient? It has been suggested that the increase in scleral field size, or eye white area (EWA), alone is enough to elicit amygdala activation (Whalen et al., 2004). In particular, Whalen and colleagues reported amygdala activity when fearful eyes were shown in a backward-masking paradigm, indicating that the amygdala can detect changes in EWA even when subjects are unaware of the stimuli being presented. The mechanism underlying the processing of EWA changes in the amygdala is unclear, although there is evidence that the amygdala can respond to coarse representations of faces made of low spatial frequency information as opposed to those depicting only high spatial frequency information (Vuilleumier et al., 2003). This implies that the amygdala may be using crude visual information in order to facilitate rapid detection and, therefore, may act as a simple detector of EWA increases since the whites of the fearful eye are extremely salient — even when seen at a distance. From an evolutionary point of view, such a mechanism could be extremely advantageous in that it is strikingly visible, can be detected rapidly, and does not require fine detail processing thus allowing for a quick evasive response if necessary. 
Yet, fear is not the only facial expression that exhibits an increase in EWA. A change in gaze direction, for example, caused by a shift in iris and pupil position can increase EWA as well. Similar to fear, gaze shifts can function as exogenous cues to indicate the presence of potential threats thus it is not surprising that the amygdala has been shown to be sensitive to gaze direction as well as gaze shifts (Kawashima et al., 1999; Hooker et al., 2003). What is not known is whether the amygdala is able to differentiate fear from other expressions that also create increases in EWA. If the amygdala responds only to fearful cues, it should activate preferentially to eyes depicting fear and not those associated with gaze shifts. On the other hand, if the amygdala relies on low spatial frequency information, as indicated by Vuilleumier et al. (2003), it may lack the capacity to distinguish between fear and other similar conditions associated with increases in EWA.

Here we used event-related fMRI to investigate how the amygdala responds to different types of EWA change. Instead of showing static images of eyes, the impression of a dynamic facial expression change was created by presenting eyes with a neutral expression both immediately before and after the presentation of each condition. Subjects viewed eye stimuli that portrayed an increase in EWA (fear, lateral shift in gaze), a decrease in EWA (happy) or no EWA change (motion control). We hypothesized that if the amygdala uses a mechanism that only detects increases in EWA, we should see similar activation to both fear and gaze shift conditions. Alternatively, if the amygdala is selective for fearful eyes exclusively, there should be a greater response to fear than to the gaze shift. 


\subsection{Methods}

\subsubsection{Participants}

Thirteen neurologically normal subjects (six female, all right-handed, aged 22-33 years) consented to participate in a study approved by the Institutional Review Board of West Virginia University. All subjects had either normal or corrected-to-normal vision. To view the forms used for subject screening and to determine handedness, see Appendix Section A.4.

\subsubsection{Stimuli}

Stimuli were selected from the JACFEE/JACNeuF series of faces by Ekman and Matsumoto (Paul Ekman Group LLC, Berkeley, CA, USA) and could belong to one of four categories (conditions): fear, gaze, happy and motion control ( Figure 4-1). All images were altered in Adobe Photoshop 7.0 with an initial conversion to grayscale and then a uniform cropping to leave a $118^{\circ} \times 38^{\circ}$ rectangle centered on the eyes. Eyebrows and other surrounding facial information were cropped out as these can act as important cues in face processing (Sadr et al., 2003). Emotional stimuli were created using six fearful and six happy faces; six gazeshifted stimuli were created from neutral faces that were altered to look either to the left or the right of the observer. All categories used six different identities and were equally balanced with respect to gender and race. Motion control stimuli were created by shifting the cropping area $0.25^{\circ}$ upwards or downwards on each face while maintaining the central positioning of the rectangle. The motion control stimuli mimicked the motion seen when the remaining three conditions transitioned from a neutral expression (see Section 4.3.3 for details about task) but unlike these other conditions, did not elicit a physical change the in facial features. Neutral eye stimuli for each facial identity for each condition were also created using the same process. Mean luminance and contrast were equated for all stimuli. Stimuli were then presented on a black background subtending $308^{\circ}$ x $238^{\circ}$ of visual angle.

Both the happy and motion control eyes served as additional stimulus conditions that would allow us to interpret the different possible mechanisms used by the amygdala when processing changes in EWA: (i) If the amygdala responds to a net change in EWA, (regardless of direction of this change), then it should also respond similarly to the fear, gaze shifts and happy conditions; (ii) if the amygdala responds to an increase in EWA only, then it should respond to 
only the fear and gaze shifts and not to happy eyes or the motion control or (iii) if the amygdala responds simply to rapid facial changes in the general vicinity of the eyes, then it should respond similarly to all conditions. While this last mechanism is inconsistent with the amygdala being selective for fear, a number of studies have suggested that the amygdala may respond to pattern motion or dynamic changes to the face (van der Gaag et al., 2007).

To determine the amount of EWA change from neutral for each of the changes in expression (fear, gaze shift and happiness), the eye white perimeter was manually traced and the number of pixels within this area was determined. For each identity, the EWA pixel difference between the neutral and condition stimuli was calculated. These pixel differences were then averaged within their respective conditions to ensure that there were no significant differences in EWA changes between the fear and gaze conditions (Figure 4-2).

\subsubsection{Task design}

The task consisted of an event-related design spanning five runs. Each run contained 40 trials, and only one of the 4 conditions was shown per trial (Figure 4-3). Each trial began with a red circle or square (both $5.58^{\circ}$ x $5.58^{\circ}$ ) presented centrally for $500 \mathrm{~ms}$. At the offset of the shape, a train of eye stimuli were presented for 1900 ms: neutral eyes first appeared for 300$1200 \mathrm{~ms}$ and were then followed by one of the conditions for $400 \mathrm{~ms}$, with the neutral eyes appearing again for the remaining 300-1200 ms. At the end of each trial, a response screen appeared prompting subjects to press a button indicating which shape they saw at the beginning of the trial. Within a single trial, the identity of the condition stimulus matched that of the neutral eyes presented immediately before and after each condition; this allowed for a smooth transition between the eye stimuli and also limited motion. Trials were separated by an intertrial interval consisting of a black screen for 2-7 s. Each condition was shown 50 times across all 5 runs [50 fear; 50 gaze (25 left, 25 right); 50 happy; 50 motion control (25 up, 25 down)]. Stimuli were delivered using Presentation software (Version 9.90, Neurobehavioral Systems, Albany, CA, USA) through Avotec Silent Vision 4000 fiber-optic eyepieces (Avotec Inc., Stuart, FL, USA) mounted on the scanner headcoil.

Subjects were told that they would see images of eyes and were not given any further information about these images. Instead, they were instructed to focus on the (task relevant) shapes presented at the beginning and end of each trial as well as to fixate on the center of the 
screen. Instructing subjects to actively search for information within eyes and faces can alter or bias activation in the amygdala (Hooker et al., 2003; Phillips et al., 2004), and to avoid this possible confound a delayed match-to-sample shape task was used for this experiment. At the end of the experiment, participants were debriefed about their viewing experience and showed no consistency in the ability to report the presence of emotions from the eye stimuli, suggesting that they were indeed paying attention to the delayed match-to-sample task involving non-eye stimuli and were not aware of the aims of the experiment.

\subsubsection{Stimuli presentation and eye tracking}

Subjects viewed the experimental task through Avotec Silent Vision 4000 fiber-optic eyepieces (Avotec Inc., Stuart, FL, USA) that were mounted on the scanner headcoil. In addition to serving as a means to present stimuli, it also allowed the eye movements of fMRI subjects to be tracked during the experiment and to ensure they were attending to the center of the screen. This eye tracking system monitored both eyes simultaneously by recording eye movements (fixations and saccades) and changes in pupil dilation. Many eye trackers, including the model employed here, use points of light that reflect off of the retina to track the position of the eye. They also monitor the location and outline of the pupil; this is accomplished by using a movable viewer to frame in each eye of the subject so that the pupil is the darkest object within the viewer. In this study, monitoring the outline of the pupil, or changes in dilation, would enable us to see whether the subjects responded to the fearful eye stimuli with an increase in pupil dilation, as this can signal an increase in vigilance (Gloor, 1997).

The eye tracker was calibrated for each subject before the experiment was run so that the relative position of the eye in relation to the location of presented stimuli could be calculated by the eye tracker data analysis program. A specific display was used for subject calibration consisting of multiple crosshairs that were located along the outer edges of the display, in the center of the display, and at points in between. Calibration was performed by instructing the subjects to attend to each crosshair until the eye tracking software indicated that it had detected their gaze at the specified location.

Eye tracking for the first fMRI subject was successful, however this was not the case for the remaining subjects. Multiple issues hindered the collection of usable eye date. First, many subjects became drowsy at different points throughout the experiment; this resulted in eyes that 
were droopy or partially closed and thus the Avotec system was unable to be continuously track them (although it is important to note that despite the fact that some subjects appeared sleepy, performances on the behavioral tasks were near $100 \%$, indicating that they were paying attention). Similarly, as the experiment progressed it was common for subjects to blink more frequently and/or to decrease the openness of their eyes, both of which impeded efficient tracking. Another common problem that was encountered involved monitoring the pupil. Since the Avotec system tracked the pupil based on it being the darkest object within the field of the viewer, thick eyelashes or drowsy eyes would cause the system to begin tracking another area within the viewer as the pupil.

The inability to collect consistent eye tracking data from fMRI subjects resulted in an abandonment of this data for analysis purposes. A trial analysis was run on the pupil dilation data of the first subject by inputting the values into Microsoft Excel and creating a graph to track the changes over time. The cyclical nature of the pupil dilation confirmed that the eye tracking data for this subject was reliable; however we were not able to repeat this for the remaining subjects due to chunks of missing values that resulted from the problems outlined previously.

\subsubsection{Imaging procedure and analysis}

Functional whole-brain axial volumes of BOLD activity were acquired on a 3 Tesla Horizon LX MRI scanner (GE Medical Systems, Milwaukee, WI, USA). Twenty-two axial slices (4mm thick, $1 \mathrm{~mm}$ gap) were obtained using the following parameters: TE/TR=25/2000s; field of view $(\mathrm{FOV})=240 \mathrm{~mm}$ (in-plane resolution $=1.875 \mathrm{~mm}^{2}$ ); bandwidth $=125$. We also acquired high resolution spoil gradient-recalled volumes (SPGR (spoiled gradient recall); FOV=240mm; matrix $=256 \times 256$; voxel size $=1.2 \mathrm{mmx} 0.9375 \mathrm{~mm} \times 0.9375 \mathrm{~mm} ; 124$ slices with $50 \%$ overlap). Functional images were acquired using a gradient echo spiral in-out sequence (Glover and Law, 2001) for 240 volumes/run. Reconstructed functional images were composed of spiral in-out trajectories, optimizing sampling from brain regions, such as the amygdala, that are prone to susceptibility artifacts and MR signal dropout. For more details about preliminary scanning to obtain these optimal parameters, see Appendix Section A.5; for more information about fMRI in general, see Appendix Section A.1.

Data were analyzed using SPM2 (Wellcome Department of Imaging Neuroscience, London, UK). Functional volumes were coregistered to anatomical images and then corrected for 
motion and slice-timing differences. The SPGR volume was normalized to the Montreal Neurological Institute (MNI) template and resliced to $2 \mathrm{~mm}^{3}$ isovoxel resolution. The parameters determined for this normalization and reslicing were applied to functional images. Data from the functional volumes were smoothed with an 8mm full width half maximum (FWHM) Gaussian kernel. A high-pass temporal filter of 1/128 s was applied to the fMRI data to remove any potential low frequency drifts in MR signal. In order to determine the response to each condition, we separately modeled the hemodynamic response to each stimulus type as a delta function located at the time point within each trial that each condition occurred and then convolved these time courses with a hemodynamic response function response. Also included in the regression model were six motion covariates (three translation and three rotation parameters) determined from motion correction and a constant term to account for potential drift. We examined positive responses to each of the conditions as well as contrasts between the conditions.

Hypothesis-generated search volumes of interest (VOIs) were selected for analysis. For the left and right amygdala VOIs, two $8 \mathrm{~mm}$ radius spheres were created, centered at MNI coordinates $-20,0,-20$, based on a review of previous studies that reported coordinates of activation of the amygdala to emotional expression and eye gaze (Table 4-1). The WFU_PickAtlas software (ANSIR Core; Wake Forest School of Medicine, Wake Forest, NC, USA) was then used to create VOIs for each fusiform gyrus (FG), frontal (orbital and inferior) cortex (Inf/OFC), intraparietal sulcus (IPS) and superior temporal sulcus (STS) in order to examine the response to the different eye-change stimuli in regions that have been demonstrated to be modulated by emotional facial expressions (Narumoto et al., 2001; for review, see Adolphs, 2002). For pictures of PickAtlas VOIs, see Appendix Section A.2.

For the amygdala VOIs, the mean response to each stimulus type relative to baseline was examined using a significance threshold of $\mathrm{p}<0.05$ (corrected for the search VOI). For comparisons between conditions in the amygdala, voxels that showed a positive response for each condition vs baseline at $\mathrm{p}<0.05$ (uncorrected) were identified, and then contrasts between conditions were thresholded at $\mathrm{p}<0.05$ (uncorrected) and more than four contiguous voxels. A liberal threshold for examining the differences between conditions within the amygdala was chosen in order to minimize the risk of Type II errors, given that this region and the possibility of its selectivity were the subject of the explicit hypotheses of this study. By only comparing positive responses to each of the stimuli we minimized the risk that differences found were due 
to negative responses to one or more of the eye-change conditions. As the other VOIs we examined were not subject to explicit hypotheses, more conservative thresholds were used. For VOIs other than the amygdala, the four conditions relative to baseline were compared using a significance threshold of $\mathrm{p}<0.05$ (corrected for search VOI). For comparisons between conditions, voxels that showed a significant positive response for each condition vs baseline at $\mathrm{p}<0.05$ (uncorrected) were included. Contrasts between the conditions were then thresholded for significance at $\mathrm{p}<0.05$ (corrected for search VOI) and more than four contiguous voxels.

The data were interpreted across all subjects; thus the results presented here are for all 13 subjects (this is the same for Experiments 3 and 4 and their respective subject pools). While individual data was not analyzed to look for differences between conditions, data processing requires that each individual's data be compiled before an across-subjects analysis can be started. Therefore, each subject's data was visibly examined for any large deviations in brain activity and it can be concluded that the results presented here (and also in Experiments 3 and 4) are not due to extreme data variances from one or two subjects in each pool. 


\subsection{Results}

\subsubsection{Behavioral Data}

Percent accuracy on the delayed match-to-sample task was high and did not differ as a function of condition (mean sd: fear $=99.54 \pm 0.88$; gaze shift $=99.69 \pm 0.75$; happy $=99.03 \pm 1.59$; motion control $=99.54 \pm 0.88 ; \mathrm{F}[3,36]=0.99, \mathrm{p}>0.40)$. Reaction time (ms) also did not vary as a function of condition (mean sd: fear $=557.78 \pm 154.10$; gaze shift $=555.78 \pm 154.10$; happy $=551.68 \pm 127.54 ;$ motion control $=556.25 \pm 139.11 ; \mathrm{F}[3,36]=0.82, \mathrm{p}>0.50)$.

\subsection{2 fMRI Activation}

\subsection{2a Amygdala}

We first examined the amygdala responses to EWA differences by analyzing the blood oxygenlevel dependent (BOLD) response to each of the four conditions. The left amygdala activated only to fearful eyes ( $\mathrm{n}=3$; paired t-tests one-tailed; $\mathrm{p}$-values corrected for multiple comparisons within amygdala VOI; Figure 4-4). Contrasts confirmed that the left amygdala response to fear was significantly greater than the responses to any other condition (p-values uncorrected; Table 1). Somewhat unexpectedly, the right amygdala responded to all conditions ( $p$-values corrected; Figure 4-4). Contrasts between conditions indicated that there were no significant differences between fear and other conditions in the right amygdala (p-values uncorrected). Our data clearly showed differences in the way the amygdala processes eye information in order to detect changes in EWA, in particular those associated with fear. To further examine this observation using a post hoc analysis, we took the average b-values describing the response for each subject to each of the four conditions from voxels in the left amygdala that showed significant differences between fear and any of the other conditions at the group level using an uncorrected significance level of $\mathrm{p}<0.05$. We then took the average $b$-values for each subject for each of the four conditions from voxels from the corresponding location in the right amygdala. A two-way analysis of variance (ANOVA) revealed a significant Hemisphere $\mathrm{x}$ Condition interaction $(\mathrm{F}[3,36]=2.92, \mathrm{p}<0.04)$. The main effect of Hemisphere was significant $(\mathrm{F}[1,12]=6.70, \mathrm{p}<$ 0.02), indicating that the overall response to the four conditions was consistently larger in the right amygdala than in the left. Additionally, the main effect of Condition was significant 
$(\mathrm{F}[3,36]=3.17, \mathrm{p}<0.03)$, which was not surprising as this factor contributed to the selection of voxels for the left amygdala.

\subsection{2b Fusiform Gyrus}

We examined the behavior of other brain regions also known to be involved in gaze and/or face processing to determine whether hemispheric differences between conditions existed outside the amygdala, including the FG. Here, we found bilateral activation to all conditions relative to baseline (p-values corrected; Figure 4-5A). In the left FG, contrasts between the conditions indicated that the response to fearful eyes was greater than to all other conditions (p-values corrected) similar to what was observed in the left amygdala. However, unlike the left amygdala, the left FG responded to all conditions, not just to fear. In the right FG, the difference between fear and the other conditions was not significant (p-values corrected). Once again, we compared the left and right FG in the same manner as the amygdala by extracting the average bvalues from each subject for each of the conditions from voxels in the left FG that showed a significant difference between fear and any of the other conditions, and voxels from the corresponding location in the right FG. Two-way ANOVA revealed an interaction between Condition and Hemisphere that trended towards significance $(F[3,36]=2.57, \mathrm{p}=0.07)$. The main

effect of Condition was significant $(F[3,36]=2.87, \mathrm{p}<0.05)$, as expected, but the main effect of Hemisphere was not $(\mathrm{F}[1,12]=2.44, \mathrm{p}>0.1)$.

\subsection{2c IPS, STS, Inf/OFC}

We found bilateral activation to all conditions in the left and right IPS (p-values corrected; Figure 4-5B); the center of activation for the left IPS was located in the posterior portion of the VOI while the center of activation for the right IPS was more anterior within the VOI. In the left posterior IPS (pIPS), contrasts between conditions indicated significant differences between fear and gaze but not fear and motion control (p- values corrected). Hence, the left hemisphere showed a degree of differentiation between conditions. Contrasts between the conditions in the right anterior intraparietal sulcus (aIPS) did not reveal differences between conditions (p-values corrected), similar to the right FG and amygdala. Finally, activation in both the left and right STS and the left and right Inf/OFC was not significantly modulated by 
Condition ( $\mathrm{p}<0.05$, corrected; Figure 4-6), and responded robustly and similarly in both hemispheres. 


\subsection{Discussion}

There is evidence to suggest that the amygdala uses simple EWA increases to detect the presence of fear in the face of another (Whalen et al., 2004), however such a mechanism indicates that the amygdala might respond to other increases in EWA that are not associated with fear. Our data suggest distinct differences in how the left and right amygdala detect such changes in EWA. The right amygdala showed a significant response to fear and gaze shifts, which were closely matched for EWA increase, as well as to happy and motion control eyes where EWA decreased or did not change, respectively. Furthermore, there was no significant difference between the strength of response to these conditions in the right amygdala. In contrast, the left amygdala showed a significant response only to fear and this activation was greater relative to that of the other conditions. A post hoc comparison revealed that there were hemispheric differences in the selectivity of the amygdala to changes in the eye region associated with different expressions. These results provide evidence that the right amygdala may act as a coarse detector of eye change, regardless of the emotional and behavioral significance behind the change. In contrast, the left amygdala showed selectivity to eye changes typically associated with fear, suggesting that the activation may be driven by more than just increases in scleral field size and that other features, such as iris and pupil position, may also contribute to the response.

A number of studies have reported unilateral activity in the amygdala, yet the issue of laterality is often not directly addressed due to differences in individual experiments with respect to stimuli, task-design and data analysis. Thus, it remains unclear how the left and right amygdala roles differ with respect to processing emotional information. Nonetheless, our data are consistent with previous fear recognition experiments that both directly and indirectly demonstrate differences between the left and right amygdala. Morris et al. (1999) show the right amygdala rapidly and non-selectively detects stimuli that pose a potential threat to the observer. Additionally, it can mediate the processing of emotional stimuli without awareness (Morris et al., 1999), can be activated by any arousing stimulus (Glascher and Adolphs, 2003) and habituates faster than the left amygdala (Wright et al., 2001), affirming the lack of selectivity by the right amygdala and suggesting that it acts as a general detector of overall change. The left amygdala, on the other hand, has indirectly been shown to discriminate between different emotional expressions (Morris et al., 1996; Kim et al., 2003; Whalen et al., 2004) and its response to fearful eyes can be mediated by the facial context in which the eyes appear (Morris et al., 2002). It has 
also been shown to be sensitive to the interaction between gaze direction and emotional expression (Adams et al., 2003), illustrating a higher level of discrimination compared with that of the right amygdala.

Additionally, overall greater activation in the right amygdala than the left to fearful faces compared with neutral faces has been reported in fMRI studies (Noesselt et al., 2005). Such findings are congruous with behavioral results indicating that subjects are faster at identifying fearful vs neutral faces when they are presented to the left visual field (Benowitz et al., 1983). Consistent with these findings, we found mainly that the response to fearful eyes was greater in the right hemisphere than the left hemisphere however, the more selective response in the left compared with the right suggests that the role of each hemisphere in threat processing is more complicated than has been previously considered. It is possible that the right amygdala activates to all conditions simply because a change is occurring to the eyes, a notion supported by an emotional information processing model proposed by Glascher and Adolphs (2003). This model suggests that the right amygdala acts in an automatic, rapid manner and is responsible for initiating a general level of arousal in response to stimuli. Such a mechanism of detection could be mediated by subcortical inputs from the superior colliculus and pulvinar into the amygdala (Amaral and Insausti, 1992) as these projections would be primarily magnocellular with a strong preference for low spatial frequency visual information (Bisti and Sireteanu, 1976; Vuilleumier et al., 2003). This would allow for the detection of coarse eye changes-i.e. the occurrence of EWA changes - but not provide the sufficient spatial detail that would allow for discrimination between similar conditions, such as fear and a shift in gaze, where EWA increases. The same model by Glascher and Adolphs (2003) posits that the left amygdala is more involved in the representation of stimuli that are emotionally stimulating and can better differentiate between stimuli that display varying levels of arousal. The hypothesis of functional laterality within the amygdala is further supported by a unilateral lesion study indicating that patients with right-sided amygdala lesions had no trouble perceiving backwardly masked fearful faces while those patients with left-sided lesions could not reliably detect these same faces (Palermo et al., 2007).

While the above evidence supports our current findings, the question of what could cause the left amygdala to be selective only to fearful eyes remains. We examined our data for other brain regions that showed analogous patterns of specificity and are known to be modulated by emotion as potential areas with which the amygdala could communicate. The FG, a cortical 
region that has long been implicated in the processing of facial information (Puce et al., 1995; Kanwisher et al., 1997), exhibited a similar response pattern to that of the right and left amygdala in that the right FG activation did not significantly vary between conditions-although unlike the left amygdala it also responded to the other conditions-while the left FG showed a significantly greater response to fear relative to the other conditions. A similar pattern was also observed in the left pIPS.

Given this similarity in the left FG and left amygdala response, it is possible that these two regions work in tandem to process eye information on a more detailed level. Because the pathway from the lateral geniculate nucleus to the visual cortex receives fine-grained inputs, the FG could be supplied with the spatial detail needed for the processing of facial features (Merigan et al., 1991). In non-human primates, there are clear anatomical connections from area TE to the amygdala and connections from the amygdala to multiple regions in extrastriate visual cortex (Iwai et al., 1987). The existence of such connections in humans are supported both by imaging studies of patients with amygdala lesions (Vuilleumier et al., 2004) and those showing a correlation of fusiform and amygdala activation to fearful faces in healthy subjects (Morris et al., 1998). The traditional view of face processing posits that visual information first travels through higher-level visual areas, such as the STS and FG, and then progresses forward to the amygdala (for a review, see Adolphs and Spezio, 2006). More recent neuroimaging studies indicate that the amygdala may actually exert influence on many stages of visual processing and in turn modulate activity in the FG, not the other way around (Morris et al., 1998a; Vuilleumier et al., 2003). Vuilleumier et al. (2003) illustrated this by using low- and high-pass filtered faces to see how this information affected amygdala and FG activation. While the FG alone activated selectively to faces made of high spatial frequency information, its activity was modulated by the low spatial frequency-driven response of the amygdala, demonstrating that the direction of information appears to flow from the amygdala to the FG. Given the evidence illustrating that the amygdala and FG actively communicate, it is possible this differs between the left and right hemisphere, potentially explaining the variation in activation between the two amygdalae.

The present study provides new insights into how the human brain detects the presence of threat using information from another's eyes. We suggest that the left and right amygdala differ in terms of their selectivity to changes in the eyes as well as to the presence of EWA increases. A coarsely tuned mechanism (right amygdala) would allow for the rapid detection of possible 
danger sources while at the same time, a finely tuned and detailed mechanism (left amygdala) would provide a more accurate determination of whether the potential threat is real. The present study not only lends more evidence to the existence of such parallel mechanisms, but also highlights a substantial difference between the response of the left and right amygdala to changes in the eyes. The interaction between presumably rapid subcortical pathways that provide crude detail about threatening stimuli and the slower, cortically mediated pathways that provide greater detail is a topic that is clearly in need of further exploration. 


\subsection{Figures and Tables}

Figure 4-1 (8)

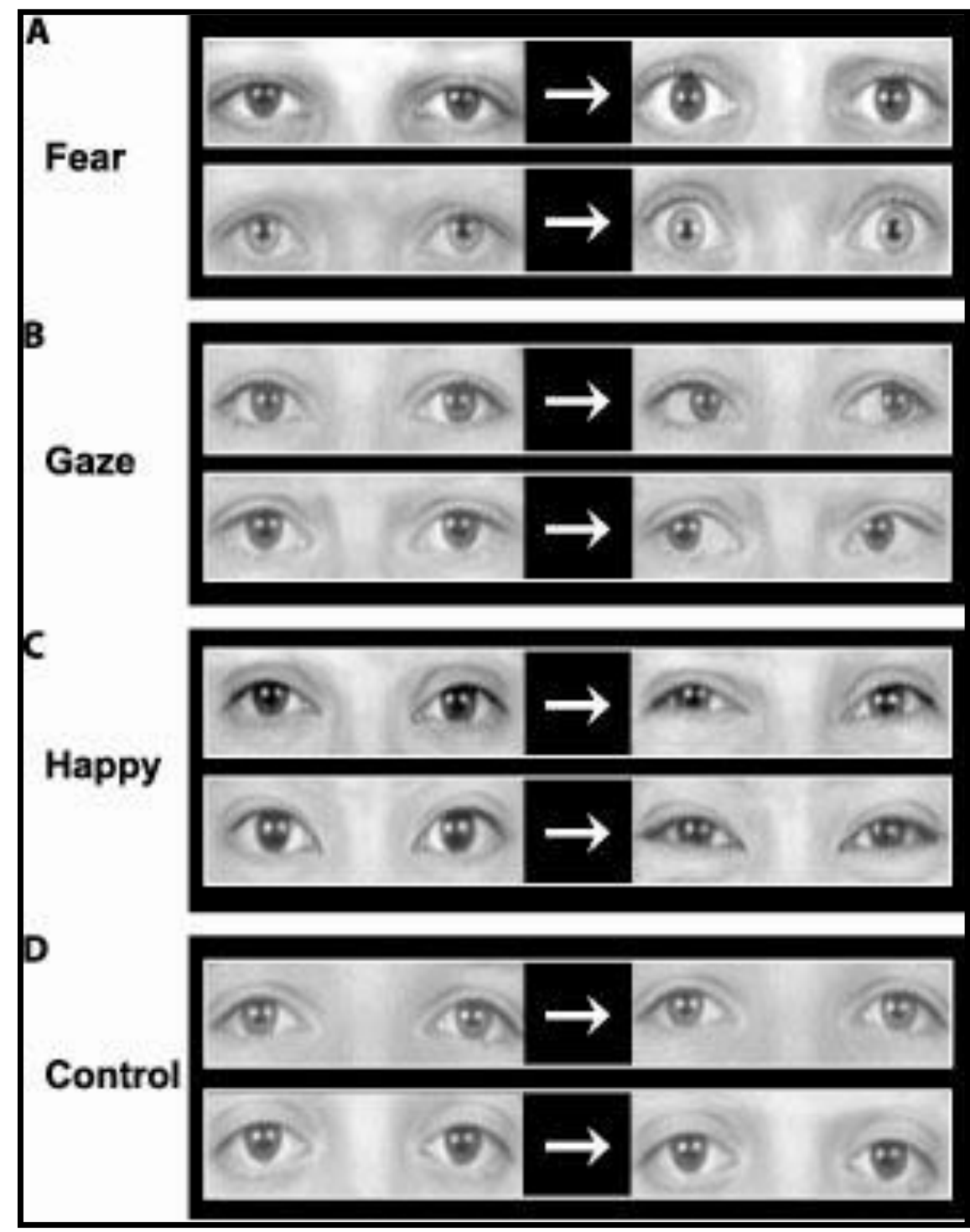

Figure 4-1. Samples showing representative stimuli from each condition. As the presentation of each condition (right column) was preceded and followed by matching neutral stimuli, the corresponding neutral images (left column) are shown to emphasize the change in EWA that occurred within a trial. A. Two sample stimuli representing the fear condition. B. Two sample stimuli representing a right (top image) and left (bottom image) shift in gaze. C. Two sample stimuli representing the happy condition. D. Two sample stimuli representing the control condition, illustrating a shift of 7 pixels up (top image) or down (bottom image). 
Figure 4-2 (9)

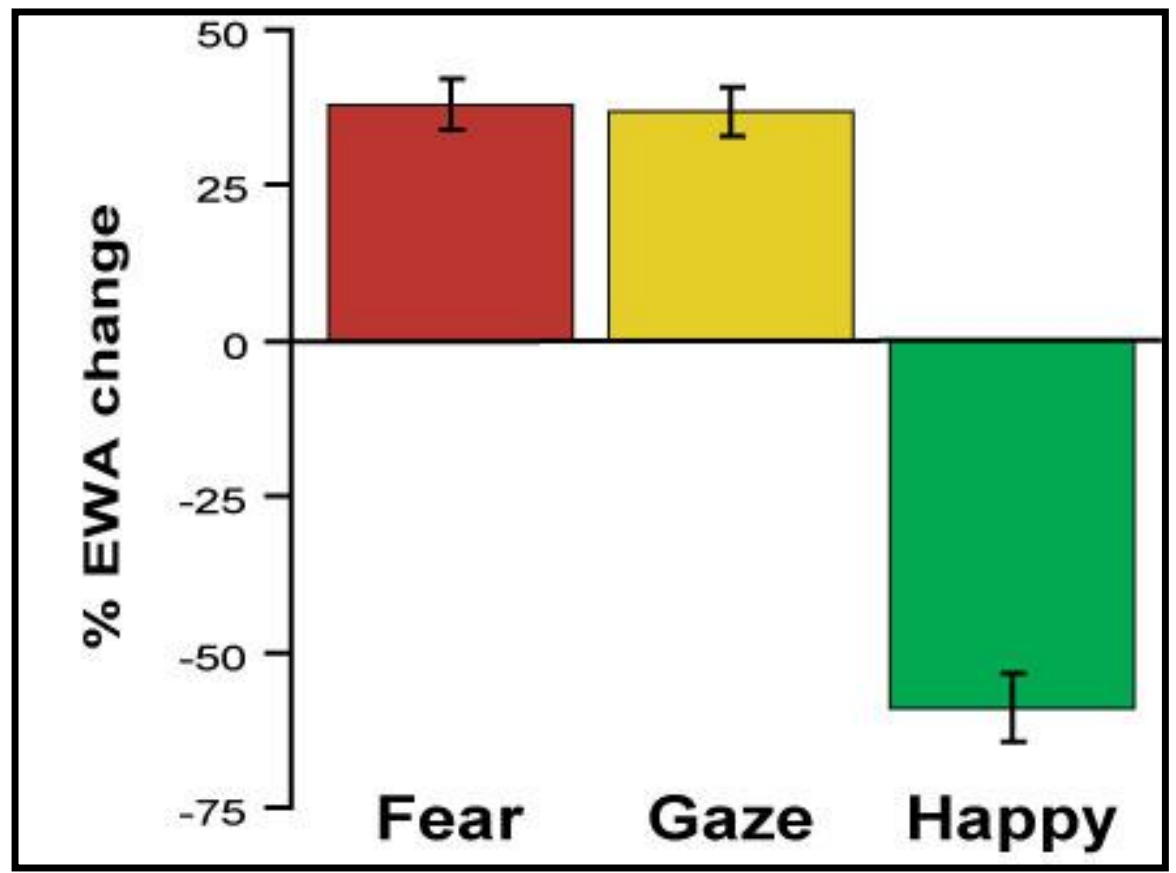

Figure 4-2. Mean ( \pm sem, $n=6$ ) percent eye white area (EWA) change from neutral plotted as a function of condition. Fear and surprise were not significantly different from one another but were significantly different from happy. As the motion control consisted of a vertical translation of neutral eyes, there was no net change in EWA. 
Figure 4-3 (10)

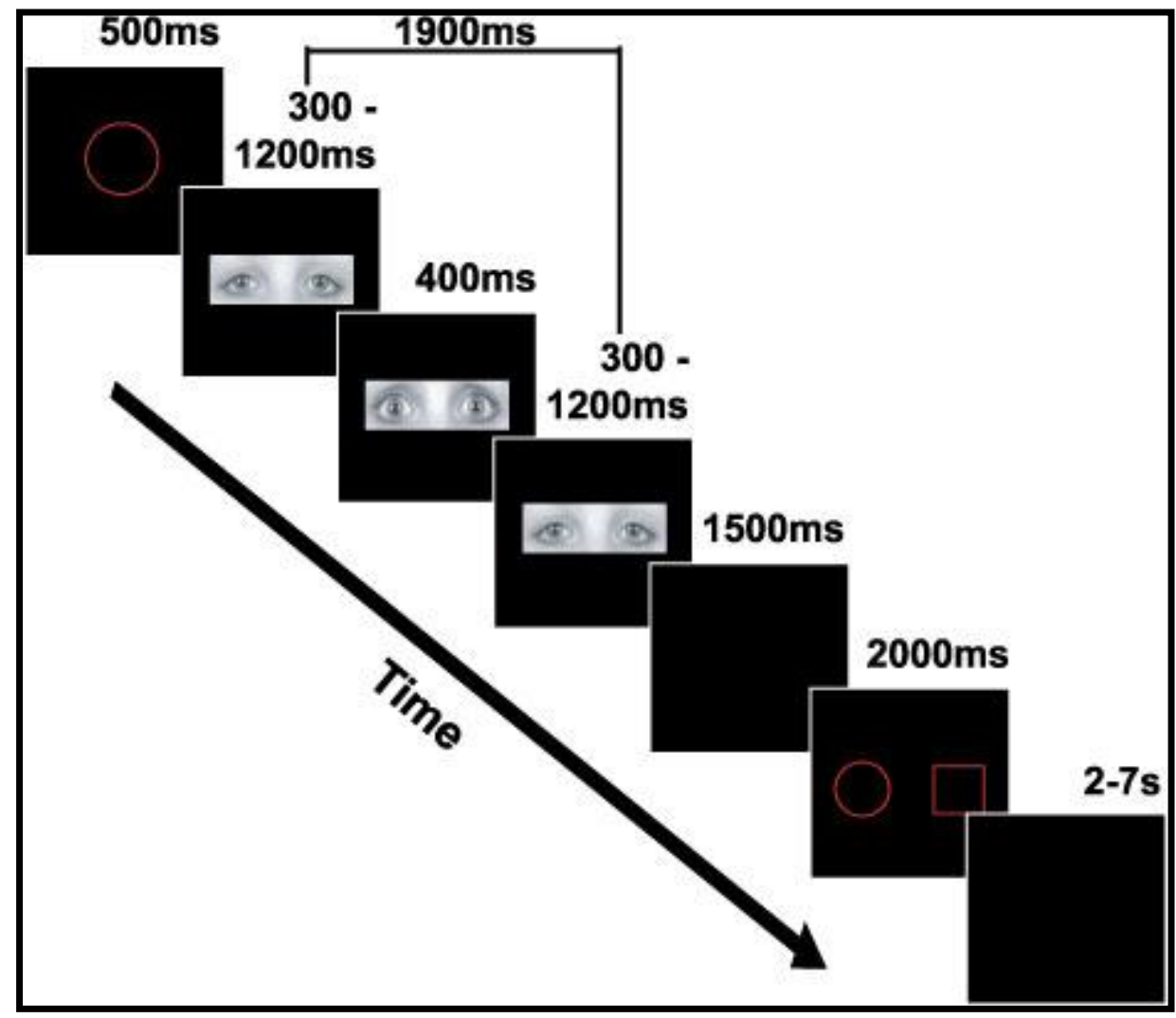

Figure 4-3. Example of a single trial. Subjects viewed one of the four conditions, in this case fear, between sets of neutral eyes, all presented for a total of $1900 \mathrm{~ms}$. Eye stimuli were presented during the delay period of a simple match-to-sample task. 
Figure 4-4 (11)

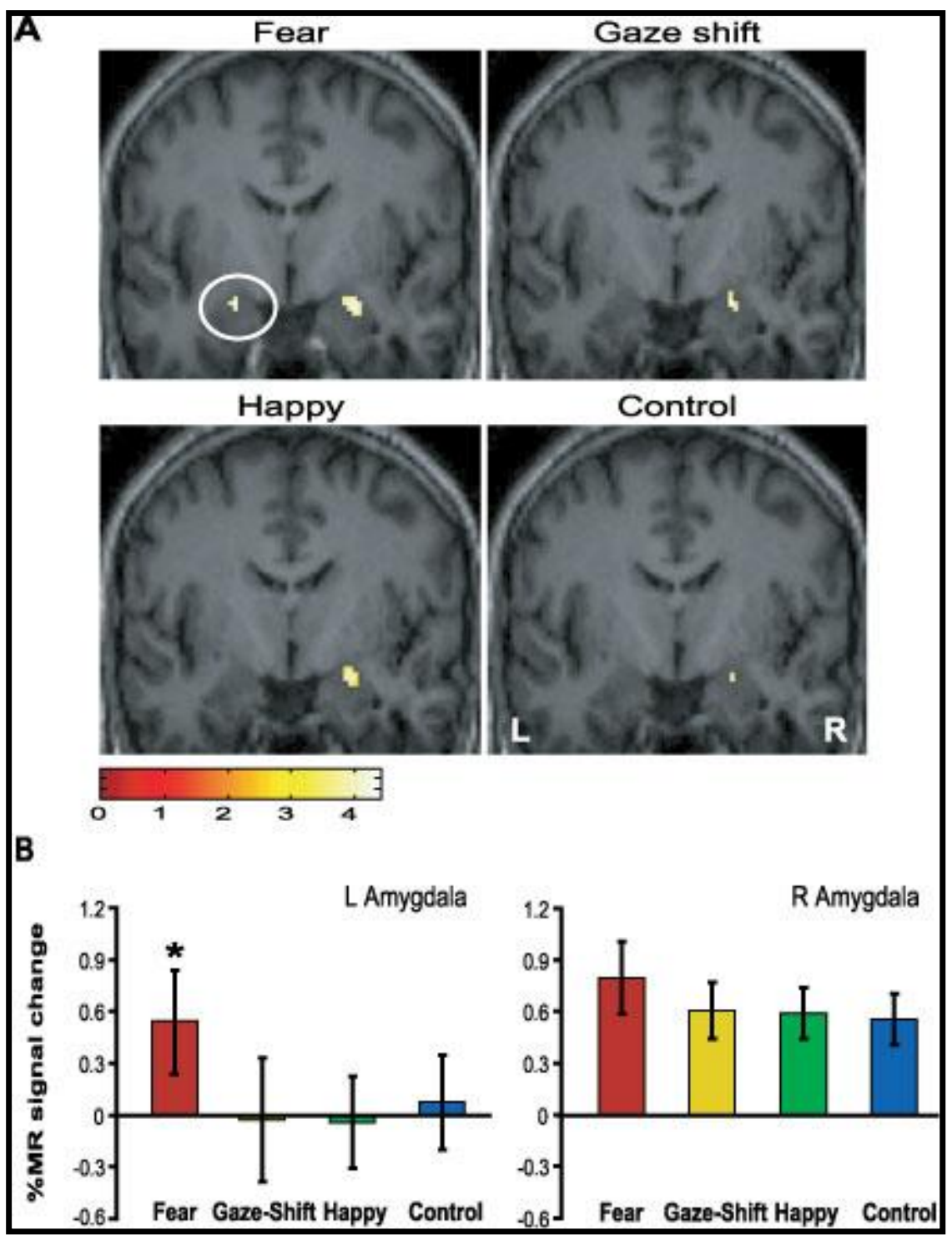

Figure 4-4. Amygdala group activation patterns and percent MR signal change. A. Left and right amygdala activation compared to baseline. Left amygdala activated only to the fearful eye condition (top left panel) whereas the right amygdala responded to all conditions (all panels). Activation is overlaid on a coronal slice (MNI coordinate: $y=-4$ ) from a representative subject. Color scale at bottomr indicates t-values. $\mathrm{L}=\mathrm{Left} ; \mathrm{R}=\mathrm{Right}$. $\mathbf{B}$. Percent MR signal change from local cluster maxima for VOIs in the left $(x=-22, y=6, z=-$ $18)$ and right $(\mathrm{x}=24, \mathrm{y}=-6, \mathrm{z}=-18)$ amygdala. 
Figure 4-5 (12)

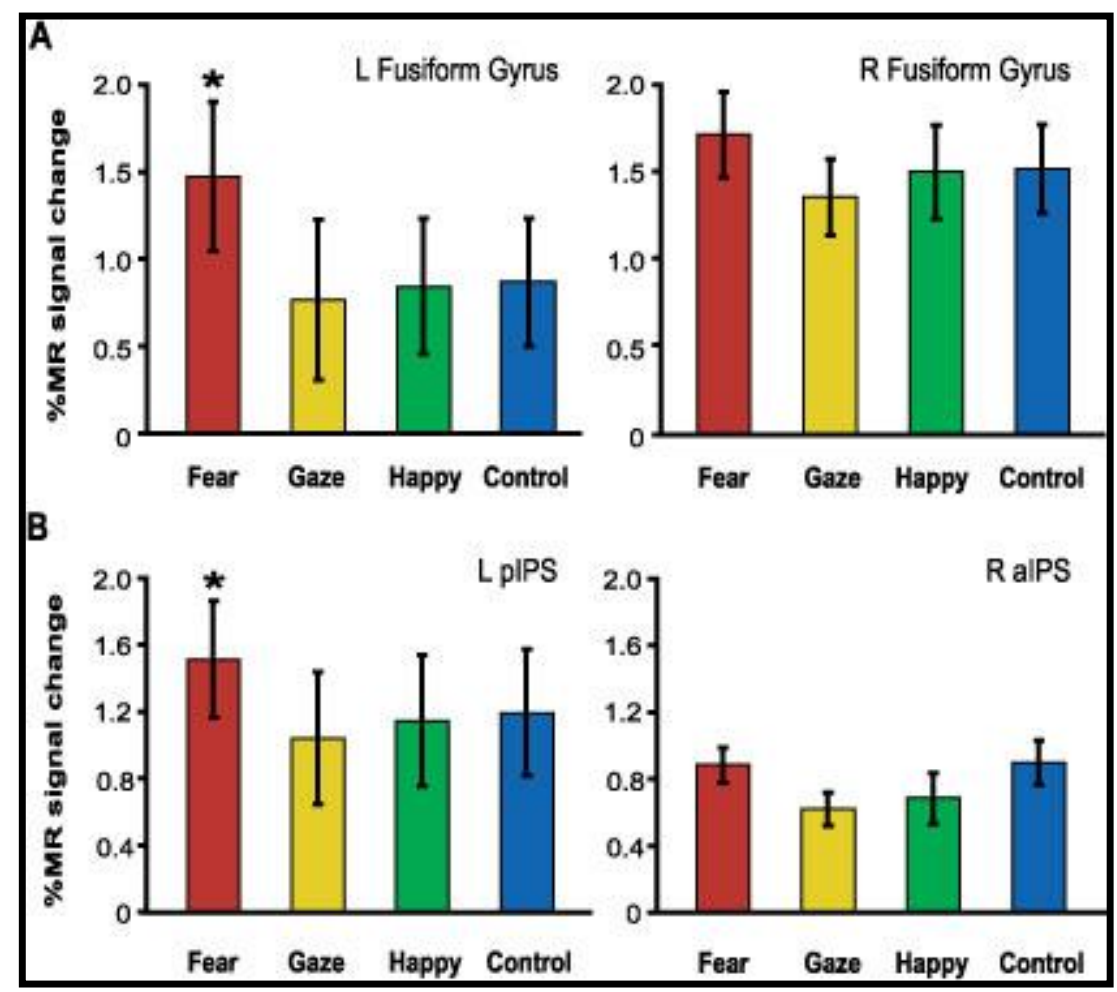

Figure 4-5. Percent MR signal change from fusiform gyrus (FG) and intraparietal sulcus (IPS). A. Percent MR signal change from local cluster maxima for VOIs in the left $(\mathrm{x}=-32, \mathrm{y}$ $=-58, \mathrm{z}=-20)$ and right $(\mathrm{x}=36, \mathrm{y}=-44, \mathrm{z}=-24)$ FG. B. Percent MR signal change from local cluster maxima for VOIs in the left posterior IPS $(x=-18, y=-86, z=-4)$ and right anterior IPS $(\mathrm{x}=34, \mathrm{y}=-38, \mathrm{z}=-50)$. 
Figure 4-6 (13)

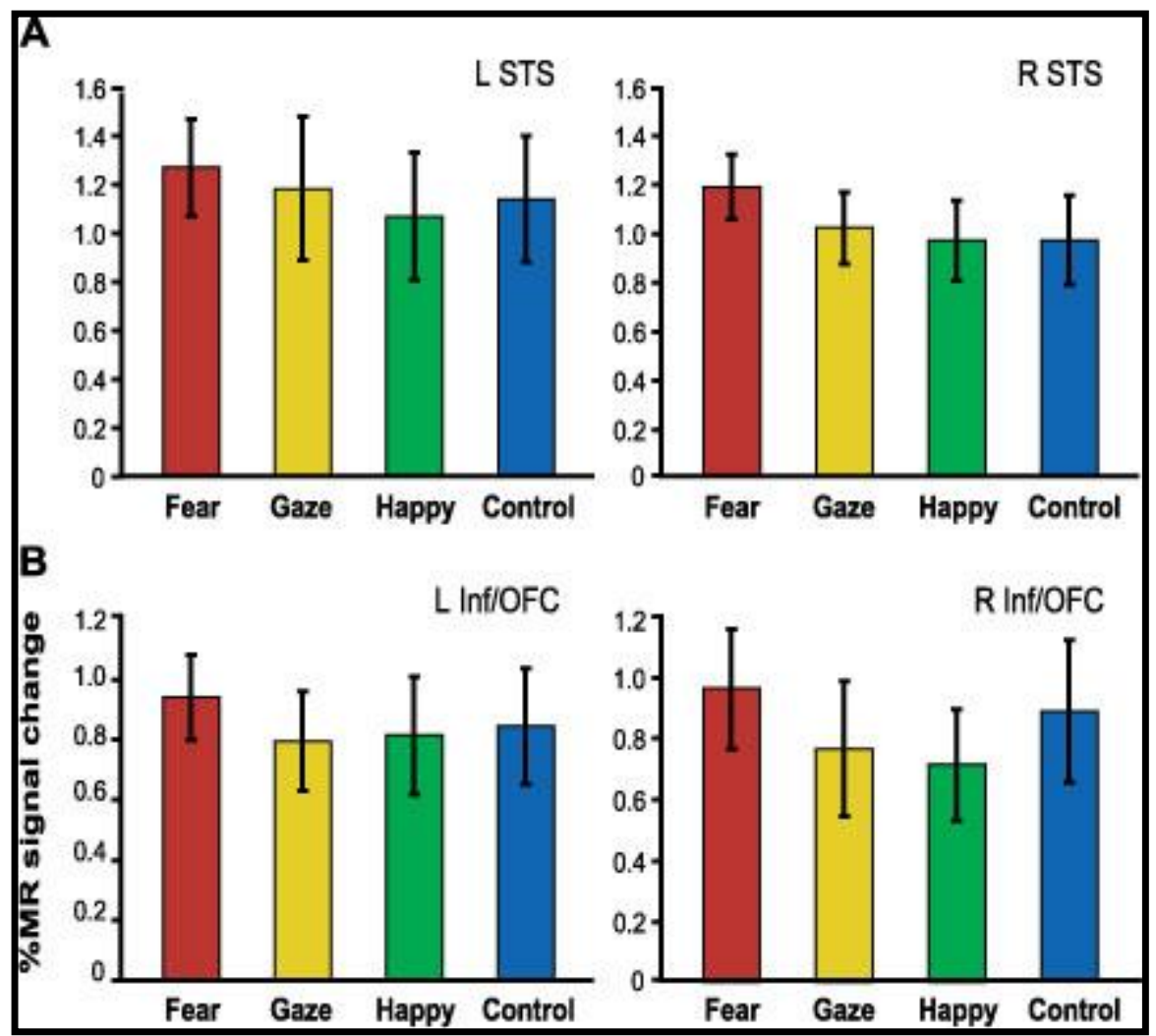

Figure 4-6. Percent MR signal change in superior temporal sulcus and inferior/orbital frontal cortices. A. Percent MR signal change from peak activation as a function of stimulus condition in the superior temporal sulcus (STS; left: $\mathrm{x}=-60, \mathrm{y}=-58, \mathrm{z}=58$; right: $\mathrm{x}=56, \mathrm{y}=$ $-40, z=2)$. Both hemispheres showed a significant response increase $(p<0.05$, corrected) to all four eye-change conditions. No significant differences between eye-change conditions were observed in either hemisphere. B. Percent MR signal change from peak activation as a function of stimulus condition in the orbital and inferior frontal cortex (Inf/OFC; left: $x=-38$, $\mathrm{y}=28, \mathrm{z}=-4$; right: $\mathrm{x}=36, \mathrm{y}=16, \mathrm{z}=32$ ). Both hemispheres showed a significant response ( $p<0.05$ corrected) to the fear and happy conditions, while only the left Inf/OFC significantly responded ( $\mathrm{p}<0.05$, corrected) to the motion control. 
Table 4-1 List of publications and MNI coordinates used for amygdala VOI analysis

\begin{tabular}{lccc}
\hline Reference & \multicolumn{3}{c}{ MNI coordinates } \\
& $\mathbf{x}$ & $\mathbf{y}$ & $\mathbf{z}$ \\
\hline Left amygdala & & & \\
Adams et al. 2003 & -15 & 14 & -18 \\
Kawashima et al.1999* & -20 & -10 & -22 \\
Morris et al. 2002 & -18 & 4 & -10 \\
Morris et al. 2002 & -22 & -6 & -20 \\
Sato et al. 2004 & -20 & -3 & -10 \\
Whalen et al. 2004 & -15 & & -23 \\
& & 3 & \\
Right amygdala & & 2 & -27 \\
Hooker et al. 2003 & 28 & -8 & -26 \\
Kawashima et al. 1999* & 26 & & -12 \\
Morris et al. 2002 & 20 & &
\end{tabular}

* Talairach coordinates converted to MNI coordinates 


\section{CHAPTER 5: Experiment 3 - Amygdala Activation to Emotional Eyes and Eyebrows}




\subsection{Introduction}

In a previous experiment (see Chapter 4), whether the amygdala could distinguish between eyes depicting fear and eyes displaying a lateral shift in gaze was investigated, as both elicit eye white area (EWA) increases and can indicate the presence of threat. The stimuli used in Experiment 2 consisted of only the eye region of the face and did not include the eyebrows. Eyebrows can act as important cues in face processing (Sadr et al., 2003) and the main focus here was increases in EWA and not emotion per se. The resulting data indicated that the left amygdala was able to discriminate fear from a shift in gaze despite similarities in EWA, however it is not known whether the amygdala can take eyebrow information into account. The study presented in this chapter compared eyes depicting fear with surprised eyes as both yield equal increases in eye white area yet differ with respect to eyebrow configuration (Figure 5-1). Like EWA, the eyebrows can be discerned at a variety of distances as they are high-contrast stimuli important for the production of emotional and social signals (Sadr et al., 2003). Ekman and Friesen (1976) found that the expression of six basic emotions (including fear and surprise) relied heavily on the upper component of the face - the eyebrows and forehead - to convey different emotions; just changing the eyebrow configuration in a face can alter an emotional expression (Figure 5-2). Therefore, it is plausible that the amygdala, if it is a true detector of fear, takes eyebrow shape into consideration when presented with eye stimuli that are nearly identical yet differ in their eyebrow arrangement.

Additionally, results from Experiment 2 indicated a laterality effect in amygdala function as the right amygdala activated to all eye-change conditions (fear, gaze shift, happiness, motion control) equally while the left amygdala responded to fear only and thus was able to discriminate the increase in EWA in fear from that visible in gaze shifts, suggesting a higher level of discrimination. The idea of functional amygdala laterality is not novel; it has been shown that the right amygdala can rapidly and non-selectively detect stimuli that pose a threat to the observer (Morris et al., 1999), can be activated by any arousing stimulus (Glascher and Adolphs, 2003) and habituates faster than the left amygdala (Wright et al., 2001). The left amygdala, on the other hand, has indirectly been shown to discriminate between different emotional expressions (Morris et al., 1996; Kim et al., 2003; Whalen et al., 2004) and its response to fearful eyes can be mediated by the facial context in which the eyes appear (Morris et al., 2002), in line with evidence that visual information can be processed via parallel pathways, one cortical and the 
other subcortical (Corballis, 1995). Therefore, in addition to examining how the amygdala responds to eyes and eyebrows displaying fear and surprise, this study also intended to discern whether the effects of laterality and specificity differences were still present between the right and left amygdala for these facial features. 


\subsection{Methods}

Prior to the fMRI study, stimuli were tested using a behavioral experiment in order to ascertain if each emotion could be identified using only the eye and eyebrow region of the face. Two different sets of subjects were used in each experiment so there was no subject overlap between the two studies.

\subsubsection{Participants}

5.2.1a Behavioral study: Fourteen neurologically normal subjects ( 8 females, 12 right-handed, aged 23-31 years of age) consented to participate in a study approved by the Institutional Review Board of West Virginia University. All subjects had either normal or corrected-to-normal vision. To view the forms used for subject screening and to determine handedness, see the Appendix Section A.4.

5.2.1b fMRI study: Thirteen neurologically normal subjects (5 females, 1 left-handed, aged 1832 years of age) consented to participate in a study approved by the Institutional Review Board of West Virginia University. None of these subjects participated in the behavioral study described above. All subjects had either normal or corrected-to-normal vision.. A post-hoc behavioral study was run following analysis of the fMRI data using 13 naïve subjects (6 females, aged 22-29 years of age).

\subsubsection{Stimuli}

Stimuli were selected from the JACFEE/JACNeuF series of faces by Ekman and Matsumoto (Paul Ekman Group LLC, Berkeley, CA, USA) and could belong to one of four categories (conditions): fear, surprise, happiness, and motion control (Figure 5-1). All images were altered in Photoshop 7.0 with an initial conversion to grayscale and then a uniform cropping to render a $118^{\circ}$ x $38^{\circ}$ rectangle centered on the eyes. Because the selected fear and surprise stimuli are completely identical with respect to EWA (Figure 5-3) yet differ with respect to eyebrow configuration, the stimuli were cropped to include the eyebrow and eye region; extraneous hair was removed. Emotional stimuli were created using six fearful, six surprise, and six happy faces while motion control stimuli were created by shifting the cropping area $0.25^{\circ}$ 
upwards or downwards on the face while maintaining the central positioning of the rectangle. The motion control stimuli mimicked the motion seen when the remaining three conditions transitioned from a neutral expression (see Section 5.2.3 for details about task) but unlike these other conditions, did not elicit a physical change the in facial features. Stimuli were made up of different identities across all conditions. Neutral eye stimuli for each facial identity for each condition were also created using the same process outlined above. Stimuli were then presented on a black background subtending $308^{\circ}$ x $238^{\circ}$ of visual angle.

Beyond comparing how fear and surprise eyes can activate the amygdala due to their identical increases in EWA, the happy and motion control eyes served as additional conditions to examine the different mechanisms the amygdala may use when processing changes in EWA: (i) if the amygdala responds simply to changes in EWA, regardless of whether this is an increase or decrease, then it should respond similarly to the fear, surprise, and happy conditions; (ii) if the amygdala responds to increases in EWA only, then it should respond to only the fear and surprise conditions; (iii) if the amygdala is truly tuned to detect fear in another's eyes, then it should show a significantly higher response to the fearful condition over the remaining three conditions; or (iv) if the amygdala simply acts as a detector of change and responds to rapid facial changes in the vicinity of the eyes, then there will be no difference between all four conditions. While this last mechanism would not be consistent with the amygdala being selective for fear, a number of studies have suggested that the amygdala may respond to pattern motion or dynamic changes to the face (van der Gaag et al., 2007). Preferential activation to fear over surprise would indicate that the amygdala can take eyebrow configuration into consideration; if the amygdala responds to fear and surprise equally, then it truly is responding to changes in EWA and does not attend to the eyebrow region of the face.

The eye white perimeter was traced manually and the number of pixels within this area was determined in order to ascertain the amount of EWA change from neutral for each of the conditions. Then, the EWA pixel difference between the neutral and condition stimuli was calculated; these pixel differences were averaged within their respective conditions to ensure that there were no significant EWA differences between fear and surprise (Figure 5-3). 


\subsubsection{Task design}

The task consisted of an event-related design spanning five runs. Each run contained 40 trials, with only 1 of the 4 conditions shown per trial (see Figure 4-3 for reference). Each trial began with a fixation cross (behavioral study) or yellow circle or square (fMRI study; both 5.58 $\mathrm{x} 5.58^{\circ}$ ) presented centrally for $500 \mathrm{~ms}$. At the offset of the shape, a train of eye stimuli were presented for 1900ms: neutral eyes first appeared for 300-1200ms and were then followed by one of the conditions for $400 \mathrm{~ms}$, with the neutral eyes appearing again for the remaining 300 $1200 \mathrm{~ms}$. A response prompt screen would then appear (see below). Each trial was separated by an intertrial interval consisting of a black screen that appeared randomly for $2-7 \mathrm{~s}$. Each condition was shown 50 times across all 5 runs using Presentation software (Version 9.90, Neurobehavioral Systems, Albany, CA, USA).

5.2.3a Behavioral study: Subjects were told they would see images of eyes and that they should indicate, via button press, whether the eyes conveyed fear, happiness, surprise, or no emotion when the response prompt screen appeared at the immediate end of each trial. All subjects were given a chance to practice beforehand using stimuli that were not included in the actual experiment.

5.2.3b fMRI study: Subjects were told that they would see images of eyes and were not given any further information about these images. Instead, they were instructed to focus on the taskrelevant shapes presented at the beginning and end of each trial as well as to fixate on the center of the screen. Instructing subjects to actively search for information within eyes and faces can alter or bias activation of the amygdala (Hooker et al., 2003; Phillips et al., 2004). At the end of each trial, a screen prompted subjects to indicate whether a square or circle appeared at the beginning of the trial via button press, and to avoid this possible confound the delayed match-tosample shape task was used for this experiment. Participants were debriefed about their viewing experience and showed no consistency in the ability to report the presence of emotions from the eye stimuli, indicating that they were indeed paying attention to the shape task and were not aware of the aims of the experiment. For details about debriefing questions, see Appendix Section A.4. 


\subsubsection{Imaging procedure and analysis}

Functional whole-brain axial volumes of BOLD activity were acquired on a 3 Tesla (T) Horizon LX MRI scanner (GE Medical Systems, Milwaukee, WI, USA) using an 8-channel headcoil. Twenty-two axial slices ( $4 \mathrm{~mm}$ thick, $1 \mathrm{~mm}$ gap) were obtained using the following parameters: $\mathrm{TE} / \mathrm{TR}=25 / 2000 \mathrm{~s} ; \mathrm{FOV}=240 \mathrm{~mm}$ (in-plane resolution $=1.875 \mathrm{~mm}^{2}$ ); bandwidth $=125$. We also acquired high resolution spoil gradient-recalled volumes (SPGR; FOV =240mm; matrix $=256 \times 256$; voxel size $=1.2 \mathrm{mmx} 0.9375 \mathrm{~mm} \times 0.9375 \mathrm{~mm} ; 124$ slices with $50 \%$ overlap). Functional images were acquired using a gradient echo spiral in-out sequence (Glover and Law, 2001) for 240 volumes/run. Reconstructed functional images were composed of spiral in-out trajectories, optimizing sampling from brain regions prone to susceptibility artifacts and MR signal drop out. For more details about fMRI, see Appendix Section A.1.

Functional images were reconstructed offline using routines written in C (G Glover, Stanford University, CA, USA) and running under Linux. Data were analyzed using FSL (version 4.0; FMRIB Software Library, University of Oxford, UK). The SPGR volume was normalized to the Montreal Neurological Institute (MNI) template and re-sliced to a $2 \mathrm{~mm}^{3}$ isovoxel resolution. The parameters determined for this normalization and re-slicing were applied to the functional images. These functional images were then co-registered to anatomical images and then corrected for motion and slice-timing differences. The functional volumes were smoothed with a 5mm FWHM Gaussian kernel and a high-pass temporal filter of 1/128 s was applied to the data in order to remove any potential low-frequency drifts in MR signal. In order to determine the response to each condition, we separately modeled the hemodynamic response to each condition group as a gamma function located at the time point within each trial where a target occurred and then convolved these time courses with a hemodynamic response function response. Also included in the regression model were six covariates (three translation and three rotation parameters) determined from motion correction and a constant term to account for drift. We examined positive responses to each of the conditions as well as contrasts between the conditions.

Hypothesis-generated search volumes of interest (VOIs) were selected for analysis. For the left and right amygdala VOIs, two 8mm radium spheres were created, centered at MNI coordinates $\mathrm{x}=-20, \mathrm{y}=0, \mathrm{z}=-20$, based on a review of previous studies reporting coordinates of amygdala activation to emotional expressions and eye gaze (see Table 3-1). WFU_PickAtlas 
software (ANSIR Core; Wake Forest School of Medicine, Wake Forest, NC, USA) was then used to create VOIs for each fusiform gyrus (FG), parietal region (including IPS), temporal region (including STS), insula, cuneus, and frontal regions in order to examine the response to each condition in regions that have been demonstrated to be modulated by emotional facial expressions (Narumoto et al., 2001; for review, see Adolphs, 2002). For pictures of PickAtlas VOIs, Appendix A.2.

For the amygdala VOIs, the mean response to each condition relative to baseline was examined using a significance threshold of $\mathrm{p}<0.05$ that was corrected for the search VOI. For comparisons between conditions, a more liberal threshold of $\mathrm{p}<0.05$ (uncorrected) was used since the amygdala was the focus of our hypotheses. For the other VOIs, we used a more conservative threshold as these regions were not the explicit subjects of our experimental hypothesis. In these regions, such as the FG and STS, the four conditions relative to baseline were compared using a significance threshold of $\mathrm{p}<0.05$ (corrected for search VOI) and a minimum voxel count of 4; small volume corrections were calculated using a Worsley-type correction (Worsley et al., 1996). Small volume corrections restrict the search for significantly active voxels to the VOI and reduce inference errors; similarly, setting a threshold for the number of active voxels also reduces the likelihood that areas of activation appear due to error. For a more detailed description of this calculation, see Appendix Section A.3. 


\subsection{Results}

\subsubsection{Behavioral Study}

Percent accuracy on the emotion identification task varied as a function of condition only for fear vs. surprise $(\mathrm{F}[1,27]=11.8, \mathrm{p}=0.0020)$ indicating that behavioral subjects could tell the difference between fear and surprise using only the eyes and eyebrows. On incorrect trials, fear was most often identified as surprise (26.5\%), happy was most often identified as neutral (18\%), surprise was most often identified as fear (11\%). Reaction times (ms) did vary as a function of condition (surprise $>$ fear $F[1,27]=17.7, p=0.000252$; surprise $>$ happy $F[1,27]=10.2, p=$ 0.0036; see Table 5-1). It is important to note that subjects were given a choice of what emotions they were viewing: fear, surprise, happy, or neutral. The reason for this was to determine whether subjects could tell the difference between fear and surprise using only the eyes and eyebrows in order to help interpret the results from the fMRI experiment (where explicit emotion identification did not occur).

\begin{tabular}{|l|c|c|}
\hline Table 5-1 & $\begin{array}{c}\text { Average Percent Correct for } \\
\text { Behavioral Subjects }(\mathbf{n = 1 4})\end{array}$ & $\begin{array}{c}\text { Average Correct Response } \\
\text { Time(ms) }\end{array}$ \\
\hline Fear & $67.0 \pm 17.5$ & $777.1 \pm 268.2$ \\
\hline Surprise & $81.2 \pm 12.21$ & $901.0 \pm 290.9$ \\
\hline Happy & $73.5 \pm 20.0$ & $785.2 \pm 267.7$ \\
\hline
\end{tabular}

\subsection{2 fMRI Study}

5.3.2a Behavioral data: Percent accuracy on the shape identification task did not differ as a function of shape (mean sd: circle $=97.7 \pm 2.2$; square $=97.5 \pm 3.4 ; \mathrm{F}[1,13]=0.21, \mathrm{p}<$ $0.656573)$. Reaction time (ms) also did not vary as a function of condition (mean sd: circle $=$ $590.9 \pm 82.3 ;$ square $=585.0 \pm 89.9 ; \mathrm{F}[1,13]=0.30, \mathrm{p}<0.590940)$.

Post-scan debriefing of fMRI subjects revealed that while all subjects reported seeing fear, surprise, or both in the eye images, often the subjects who identified happy also said it could have been anger. To view the questions used to debrief subjects, see Appendix Section A.4. Because happy was often confused for anger, a post-experimental behavioral experiment 
was run using 13 new subjects who had not participated in any prior experiments pertaining to this study. Subjects viewed all 24 eye images from each condition, plus 4 images that were not in the previous experiments, and were asked to freely assign an emotion to the image. Happy was correctly identified as happy only $36 \%$ of the time across all subjects, and was most often labeled as angry and confused; fear was $33 \%$ correct and incorrect answers were labeled as either surprise or shock. Surprise was correctly identified as surprise $41 \%$ of the time; incorrect answers were labeled as either shock or fear. Finally, motion control was correctly identified $53 \%$ of the time, and incorrectly characterized as bored or unsure. 


\subsection{2b fMRI data}

The data presented in this section is broken up into 2 sections: primary and secondary data. The primary data section contains the VOIs that are considered to be core to face/emotion processing and/or show the same pattern of activation as the amygdala (the FG, the STS, the IPS, and the insula). The secondary data section contains VOIs that have been shown to be involved in face/emotion processing but with less consistency.

The following tables display size of activation clusters (in voxels), the MNI coordinates for these centers of activation, and the p-values for each VOI, and the percent signal change. Shaded areas represent the condition with the highest significant voxel cluster for each volume of interest. P-values of 0.000 indicate a significance level of $\mathrm{p}<0.001$. * Symbolizes significance at the corrected level. Percent of activation cluster size represents the number of active voxels per total number of voxels in the VOI. 


\subsection{2b-1 Primary Data}

Amygdala: The BOLD response to each of the four eye conditions was analyzed to examine amygdala activation to EWA differences. Both the left and right amygdala activated to all four conditions compared to baseline ( $\mathrm{p}<0.05$ uncorrected), however happiness elicited the largest amount of activation bilaterally (Table 5-2; Figure 5.4; Figure 5.5). Additionally, contrasts between the conditions indicated that there was a significant difference between happy and motion control in the left amygdala but no differences between conditions in the right amygdala.

P-values of 0.000 indicate a significance level of $p<0.001$. Symbolizes significance at the corrected level.

\begin{tabular}{|c|c|c|c|c|c|}
\hline \multicolumn{6}{|l|}{ Table 5-2 } \\
\hline $\begin{array}{l}\text { Left } \\
\text { Amygdala }\end{array}$ & $\begin{array}{c}\text { Size of } \\
\text { activation } \\
\text { cluster } \\
\text { (voxels) }\end{array}$ & $\begin{array}{r}\text { Center of } \\
\text { activation } \\
(\mathrm{MNI}) \\
(\mathbf{x}, \mathbf{y}, \mathbf{z})\end{array}$ & p-value & $\begin{array}{l}\text { Percent of } \\
\text { active voxels } \\
\text { in VOI }\end{array}$ & $\begin{array}{c}\text { Percent } \\
\text { signal } \\
\text { change }\end{array}$ \\
\hline Fear & 2 & $-20,-2,-26$ & 0.033 & $0.7 \%$ & $0.024 \%$ \\
\hline Surprise & 9 & $-16,-6,-12$ & 0.020 & $3.5 \%$ & $0.038 \%$ \\
\hline Happy & 38 & $-16,-4,-20$ & 0.004 & $14.8 \%$ & $0.099 \%$ \\
\hline Control & 5 & $-16,-6,-18$ & 0.034 & $1.9 \%$ & $0.052 \%$ \\
\hline $\begin{array}{l}\text { Happy > } \\
\text { Control }\end{array}$ & 9 & $-14,0,-22$ & 0.014 & $3.5 \%$ & $0.098 \%$ \\
\hline \multicolumn{6}{|l|}{$\begin{array}{l}\text { Right } \\
\text { Amygdala }\end{array}$} \\
\hline Fear & 1 & $16,-4,-22$ & 0.044 & $0.4 \%$ & $0.081 \%$ \\
\hline Surprise & 2 & $20,-8,-20$ & 0.038 & $0.8 \%$ & $0.088 \%$ \\
\hline Happy & 12 & $16,-4,-22$ & 0.017 & $4.7 \%$ & $0.107 \%$ \\
\hline Control & 2 & $18,-4,-26$ & 0.044 & $0.8 \%$ & $0.096 \%$ \\
\hline
\end{tabular}


5.3.2b-2 Fusiform Gyrus: The left and right fusiform gyri activated to all four conditions compared to baseline equally ( $\mathrm{p}<0.001$, uncorrected). When small volume corrections were applied, all four conditions (versus baseline) survived in both the left and right fusiform (p < 0.05, corrected; see Table 5-3). For a visual representation of activation in the fusiform, see Figure 5-6.

P-values of 0.000 indicate a significance level of $\mathrm{p}<0.001$. $*$ Symbolizes significance at the corrected level.

\begin{tabular}{|l|c|c|c|c|c|}
\hline Table 5-3 & $\begin{array}{c}\text { Size of } \\
\text { activation } \\
\text { cluster } \\
\text { (voxels) } \\
\text { Fusiform }\end{array}$ & $\begin{array}{c}\text { Center of } \\
\text { activation } \\
\text { (MNI) } \\
(\mathbf{x}, \mathbf{y}, \mathbf{z})\end{array}$ & p-value & $\begin{array}{c}\text { Percent of } \\
\text { active voxels } \\
\text { in VOI }\end{array}$ & $\begin{array}{c}\text { Percent } \\
\text { signal } \\
\text { change }\end{array}$ \\
\hline Fear & 860 & $-34,-56,-20$ & $0.000^{*}$ & $34.2 \%$ & $0.845 \%$ \\
\hline Surprise & 877 & $-34,-56,-20$ & $0.000^{*}$ & $34.8 \%$ & $0.862 \%$ \\
\hline Happy & 877 & $-36,-50,-22$ & $0.000^{*}$ & $34.8 \%$ & $0.868 \%$ \\
\hline Control & 827 & $-36,-50,-22$ & $0.000^{*}$ & $32.8 \%$ & $0.703 \%$ \\
\hline $\begin{array}{l}\text { Right } \\
\text { Fusiform }\end{array}$ & 926 & $40,-76,-14$ & $0.000^{*}$ & $40.1 \%$ & $1.077 \%$ \\
\hline Fear & 938 & $40,-76,-14$ & $0.000^{*}$ & $40.6 \%$ & $1.152 \%$ \\
\hline Surprise & 948 & $40,-76,-14$ & $0.000^{*}$ & $41.0 \%$ & $1.105 \%$ \\
\hline Happy & & $38,-70,-12$ & $0.000^{*}$ & $39.5 \%$ & $0.921 \%$ \\
\hline Control & & & & \\
\hline
\end{tabular}


Temporal Regions (including STS): Both left and right temporal regions activated to all four conditions compared to baseline ( $\mathrm{p}<0.001$, uncorrected). All conditions compared to baseline survived small volume correction at $\mathrm{p}<0.05$ (corrected; see Table 5-4). For a visual representation of activation in the STS, see Figure 5-7.

P-values of 0.000 indicate a significance level of $\mathrm{p}<0.001$. $*$ Symbolizes significance at the corrected level.

\begin{tabular}{|c|c|c|c|c|c|}
\hline \multicolumn{6}{|l|}{ Table 5-4 } \\
\hline \begin{tabular}{|l|} 
Left \\
Temporal
\end{tabular} & $\begin{array}{c}\text { Size of } \\
\text { activation } \\
\text { cluster } \\
\text { (voxels) }\end{array}$ & $\begin{array}{c}\text { Center of } \\
\text { activation } \\
(\mathrm{MNI}) \\
(\mathbf{x}, \mathbf{y}, \mathbf{z})\end{array}$ & p-value & $\begin{array}{l}\text { Percent of } \\
\text { active voxels } \\
\text { in VOI }\end{array}$ & $\begin{array}{c}\text { Percent } \\
\text { signal } \\
\text { change }\end{array}$ \\
\hline Fear & 991 & $-46,-66,-8$ & $0.000^{*}$ & $9.5 \%$ & $0.411 \%$ \\
\hline Surprise & 1104 & $-46,-58,-14$ & $0.000^{*}$ & $10.6 \%$ & $0.418 \%$ \\
\hline Happy & 1157 & $-46,-58,-14$ & $0.000^{*}$ & $11.1 \%$ & $0.471 \%$ \\
\hline Control & 1509 & $-48,-58,6$ & $0.000^{*}$ & $14.5 \%$ & $0.373 \%$ \\
\hline \multicolumn{6}{|l|}{$\begin{array}{l}\text { Right } \\
\text { Temporal }\end{array}$} \\
\hline Fear & 3336 & $48,-72,-4$ & $0.000^{*}$ & $30.0 \%$ & $0.853 \%$ \\
\hline Surprise & 3567 & $48,-72,-4$ & $0.000^{*}$ & $32.1 \%$ & $0.842 \%$ \\
\hline Happy & 3550 & $48,-72,-4$ & $0.000^{*}$ & $32.0 \%$ & $0.878 \%$ \\
\hline Control & 3808 & $48,-72,-4$ & $0.000^{*}$ & $34.3 \%$ & $0.728 \%$ \\
\hline
\end{tabular}


Insula: Both the left and right insula significantly activated to all four conditions compared to baseline ( $\mathrm{p}<0.001$, uncorrected). All conditions compared to baseline survived small volume correction in both hemispheres $(\mathrm{p}<0.05$, corrected; see Table 5-5). For a visual representation of activation in the insula, see Figure 5-8.

$\mathrm{P}$-values of 0.000 indicate a significance level of $\mathrm{p}<0.001$. $*$ Symbolizes significance at the corrected level.

\begin{tabular}{|l|c|c|c|c|c|}
\hline Table 5-5 & $\begin{array}{c}\text { Size of } \\
\text { activation } \\
\text { cluster } \\
\text { (voxels) }\end{array}$ & $\begin{array}{c}\text { Center of } \\
\text { activation } \\
\text { (MNI) } \\
\mathbf{( x , y , z )}\end{array}$ & p-value & $\begin{array}{c}\text { Percent of } \\
\text { active voxels } \\
\text { in VOI }\end{array}$ & $\begin{array}{c}\text { Percent } \\
\text { signal } \\
\text { change }\end{array}$ \\
\hline Fear & 235 & $-30,24,-4$ & $0.000^{*}$ & $12.6 \%$ & $0.183 \%$ \\
\hline Surprise & 166 & $-30,26,-4$ & $0.000^{*}$ & $8.9 \%$ & $0.152 \%$ \\
\hline Happy & 285 & $-30,26,-6$ & $0.000^{*}$ & $15.3 \%$ & $0.196 \%$ \\
\hline Control & 339 & $-30,26,-6$ & $0.000^{*}$ & $18.2 \%$ & $0.191 \%$ \\
\hline Right Insula & & & & & \\
\hline Fear & 185 & $32,22,-10$ & $0.000^{*}$ & $10.5 \%$ & $0.155 \%$ \\
\hline Surprise & 145 & $38,32,6$ & $0.000^{*}$ & $8.2 \%$ & $0.124 \%$ \\
\hline Happy & 140 & $30,22,-8$ & $0.000^{*}$ & $7.9 \%$ & $0.179 \%$ \\
\hline Control & 326 & $38,32,6$ & $0.000^{*}$ & $18.4 \%$ & $0.207 \%$ \\
\hline
\end{tabular}


Parietal Regions (including IPS): Both left and right parietal regions significantly activated to all conditions compared to baseline ( $\mathrm{p}<0.001$, uncorrected). All conditions compared to baseline survived small volume correction in both hemispheres with the exception of happy in the right parietal region $(\mathrm{p}<0.05$, corrected; see Table 5-6). For a visual representation of activation in the IPS, see Figure 5-9.

P-values of 0.000 indicate a significance level of $\mathrm{p}<0.001$. $*$ Symbolizes significance at the corrected level.

\begin{tabular}{|l|c|c|c|c|c|}
\hline Table 5-6 & $\begin{array}{c}\text { Size of } \\
\text { activation } \\
\text { cluster } \\
\text { (voxels) }\end{array}$ & $\begin{array}{c}\text { Center of } \\
\text { activation } \\
\text { (MNI) } \\
\mathbf{( x , y , ~ z )}\end{array}$ & p-value & $\begin{array}{c}\text { Percent of } \\
\text { active voxels } \\
\text { in VOI }\end{array}$ & $\begin{array}{c}\text { Percent } \\
\text { signal } \\
\text { change }\end{array}$ \\
\hline Fear & 1882 & $-52,-26,46$ & $0.000^{*}$ & $41.7 \%$ & $0.385 \%$ \\
\hline Surprise & 1679 & $-50,-26,46$ & $0.000^{*}$ & $37.2 \%$ & $0.303 \%$ \\
\hline Happy & 2107 & $-52,-26,46$ & $0.000^{*}$ & $46.7 \%$ & $0.293 \%$ \\
\hline Control & 2067 & $-50,-26,46$ & $0.000^{*}$ & $45.8 \%$ & $0.334 \%$ \\
\hline Right Parietal & & & & & \\
\hline Fear & 893 & $34,-54,48$ & $0.000^{*}$ & $25.3 \%$ & $0.432 \%$ \\
\hline Surprise & 764 & $38,-62,54$ & $0.000 *$ & $21.4 \%$ & $0.452 \%$ \\
\hline Happy & 685 & $38,-62,54$ & 0.000 & $19.2 \%$ & $0.491 \%$ \\
\hline Control & 942 & $34,-54,48$ & $0.000^{*}$ & $26.4 \%$ & $0.446 \%$ \\
\hline
\end{tabular}




\subsection{2b-2 Secondary Data}

Cingulum: Both the left and right cingulum significantly activated to all conditions compared to baseline ( $p<0.01$, uncorrected). Only fear survived small volume corrections in both hemispheres, while motion control survived in the right ( $p<0.05$, corrected). See Table 5-7.

$\mathrm{P}$-values of 0.000 indicate a significance level of $\mathrm{p}<0.001$. $*$ Symbolizes significance at the corrected level.

\begin{tabular}{|c|c|c|c|c|}
\hline \multicolumn{5}{|l|}{ Table 5-7 } \\
\hline $\begin{array}{l}\text { Left } \\
\text { Cingulum }\end{array}$ & $\begin{array}{c}\text { Size of } \\
\text { activation } \\
\text { cluster } \\
\text { (voxels) }\end{array}$ & $\begin{array}{c}\text { Center of activation } \\
\text { (MNI) } \\
\mathbf{x}, \mathbf{y}, \mathbf{z}\end{array}$ & p-value & $\begin{array}{l}\text { Percent of active } \\
\text { voxels in VOI }\end{array}$ \\
\hline Fear & 398 & $-4,2,44$ & $0.000 *$ & $10.5 \%$ \\
\hline Surprise & 238 & $-2,2,44$ & 0.000 & $6.3 \%$ \\
\hline Нарру & 162 & $-6,10,42$ & 0.001 & $4.3 \%$ \\
\hline Control & 260 & $2,12,42$ & 0.000 & $6.8 \%$ \\
\hline \multicolumn{5}{|l|}{$\begin{array}{l}\text { Right } \\
\text { Cingulum }\end{array}$} \\
\hline Fear & 339 & $2,8,44$ & $0.000^{*}$ & $8.8 \%$ \\
\hline Surprise & 154 & $2,8,44$ & 0.000 & $4.0 \%$ \\
\hline Happy & 180 & $4,16,40$ & 0.001 & $4.7 \%$ \\
\hline Control & 314 & $6,12,42$ & $0.000 *$ & $8.2 \%$ \\
\hline
\end{tabular}


Cuneus: Both the left and right cuneus significantly activated to all conditions compared to baseline with the exception of motion control in the right hemisphere ( $\mathrm{p}<0.05$, uncorrected). No conditions survived small volume corrections. See Table 5-8.

P-values of 0.000 indicate a significance level of $p<0.001$. Symbolizes significance at the corrected level.

\begin{tabular}{|c|c|c|c|c|}
\hline \multicolumn{5}{|l|}{ Table 5-8 } \\
\hline Left Cuneus & $\begin{array}{c}\text { Size of } \\
\text { activation } \\
\text { cluster } \\
\text { (voxels) }\end{array}$ & $\begin{array}{l}\text { Center of activation } \\
\text { (MNI) } x, y, z\end{array}$ & p-value & $\begin{array}{l}\text { Percent of active } \\
\text { voxels in VOI }\end{array}$ \\
\hline Fear & 14 & $-18,-64,36$ & 0.016 & $0.3 \%$ \\
\hline Surprise & 34 & $-18,-62,36$ & 0.011 & $0.7 \%$ \\
\hline Happy & 40 & $-18,-62,36$ & 0.002 & $0.8 \%$ \\
\hline Control & 19 & $-12,-36,60$ & 0.007 & $0.4 \%$ \\
\hline \multicolumn{5}{|l|}{ Right Cuneus } \\
\hline Fear & 55 & $6,-66,44$ & 0.005 & $1.2 \%$ \\
\hline Surprise & 38 & $10,-74,60$ & 0.005 & $0.8 \%$ \\
\hline Нарру & 7 & $4,-68,42$ & 0.020 & $0.1 \%$ \\
\hline Control & $\begin{array}{l}\text { no sig. } \\
\text { results }\end{array}$ & no sig. results & no sig. results & no sig. results \\
\hline
\end{tabular}




\section{Frontal Regions}

a. Pars Triangularis: Both left and right pars triangularis significantly activated to all conditions compared to baseline ( $\mathrm{p}<0.001$, uncorrected). All conditions compared to baseline survived small volume correction in both the left and right pars triangularis $(\mathrm{p}<0.05$, corrected). See Table 5-9.

P-values of 0.000 indicate a significance level of $\mathrm{p}<0.001$. $*$ Symbolizes significance at the corrected level.

\begin{tabular}{|c|c|c|c|c|}
\hline \multicolumn{5}{|l|}{ Table 5-9 } \\
\hline $\begin{array}{l}\text { Left Pars } \\
\text { Tri }\end{array}$ & $\begin{array}{c}\text { Size of } \\
\text { activation } \\
\text { cluster } \\
\text { (voxels) }\end{array}$ & $\begin{array}{c}\text { Center of activation } \\
\text { (MNI) } \\
\mathbf{x}, \mathbf{y}, \mathbf{z}\end{array}$ & p-value & $\begin{array}{l}\text { Percent of active } \\
\text { voxels in VOI }\end{array}$ \\
\hline Fear & 616 & $-52,14,28$ & $0.000 *$ & $23.4 \%$ \\
\hline Surprise & 405 & $-50,14,28$ & $0.000 *$ & $16.0 \%$ \\
\hline Happy & 877 & $-52,14,28$ & $0.000 *$ & $34.7 \%$ \\
\hline Control & 367 & $-36,24,-2$ & $0.000 *$ & $14.5 \%$ \\
\hline \multicolumn{5}{|c|}{$\begin{array}{l}\text { Right Pars } \\
\text { Tri }\end{array}$} \\
\hline Fear & 1597 & $54,32,18$ & $0.000 *$ & $74.2 \%$ \\
\hline Surprise & 1416 & $54,32,18$ & $0.000 *$ & $65.8 \%$ \\
\hline Happy & 1515 & $54,32,18$ & $0.000 *$ & $70.4 \%$ \\
\hline Control & 1723 & $54,38,8$ & $0.000 *$ & $80.1 \%$ \\
\hline
\end{tabular}


b. Pars Opercularis: Both left and right pars operculi significantly activated to all conditions compared to baseline ( $\mathrm{p}<0.001$, uncorrected). All four conditions compared to baseline survived small volume correction in both hemispheres ( $\mathrm{p}<0.05$, corrected). See Table 5-10.

$\mathrm{P}$-values of 0.000 indicate a significance level of $\mathrm{p}<0.001$. $*$ Symbolizes significance at the corrected level.

\begin{tabular}{|c|c|c|c|c|}
\hline \multicolumn{5}{|l|}{ Table 5-10 } \\
\hline $\begin{array}{l}\text { Left Pars } \\
\text { Oper }\end{array}$ & $\begin{array}{c}\text { Size of } \\
\text { activation } \\
\text { cluster } \\
\text { (voxels) }\end{array}$ & $\begin{array}{c}\text { Center of activation } \\
\text { (MNI) } \\
\mathbf{x}, \mathbf{y}, \mathbf{z}\end{array}$ & p-value & $\begin{array}{l}\text { Percent of active } \\
\text { voxels in VOI }\end{array}$ \\
\hline Fear & 513 & $-48,10,28$ & $0.000 *$ & $49.4 \%$ \\
\hline Surprise & 481 & $-48,10,28$ & $0.000^{*}$ & $46.3 \%$ \\
\hline Hарру & 653 & $-46,10,20$ & $0.000 *$ & $63.0 \%$ \\
\hline Control & 541 & $-48,8,28$ & $0.000^{*}$ & $52.1 \%$ \\
\hline \multicolumn{5}{|c|}{$\begin{array}{l}\text { Right Pars } \\
\text { Oper }\end{array}$} \\
\hline Fear & 932 & $54,10,30$ & $0.000^{*}$ & $66.6 \%$ \\
\hline Surprise & 933 & $54,10,28$ & $0.000 *$ & $66.6 \%$ \\
\hline Happy & 962 & $54,12,30$ & $0.000 *$ & $68.8 \%$ \\
\hline Control & 1166 & $40,6,34$ & $0.000^{*}$ & $83.3 \%$ \\
\hline
\end{tabular}


c. Middle Frontal Gyrus: Both left and right gyri significantly activated to all conditions compared to baseline ( $\mathrm{p}<0.001$, uncorrected). All conditions compared to baseline survived small volume correction in the right hemisphere, only fear and happy survived in the left hemisphere ( $p<0.05$, corrected). See Table 5-11.

P-values of 0.000 indicate a significance level of $\mathrm{p}<0.001$. $*$ Symbolizes significance at the corrected level.

\begin{tabular}{|lcccc|}
\hline Table 5-11 & $\begin{array}{c}\text { Size of } \\
\text { activation } \\
\text { cluster } \\
\text { (voxels) }\end{array}$ & $\begin{array}{c}\text { Center of activation } \\
\text { (MNI) }\end{array}$ & $\begin{array}{c}\text { p-value } \\
\mathbf{x}, \mathbf{y}, \mathbf{z}\end{array}$ & $\begin{array}{c}\text { Percent of active } \\
\text { voxels in VOI }\end{array}$ \\
Fear & 171 & $-50,12,44$ & $0.000^{*}$ & $3.5 \%$ \\
Surprise & 89 & $-50,14,42$ & 0.000 & $1.8 \%$ \\
Happy & 251 & $-50,14,42$ & $0.000^{*}$ & $5.2 \%$ \\
Control & 127 & $-52,14,44$ & 0.000 & $2.6 \%$ \\
Right Mid & & & & \\
Fear & 913 & $38,-6,58$ & $0.000^{*}$ & $17.9 \%$ \\
Surprise & 775 & $42,-6,52$ & $0.000^{*}$ & $15.2 \%$ \\
Happy & 711 & $38,-6,58$ & $0.000^{*}$ & $14.0 \%$ \\
Control & 897 & $42,4,40$ & $0.000^{*}$ & $17.6 \%$ \\
\hline
\end{tabular}


d. Supraorbital Sulcus: The right superior orbital region significantly activated to all conditions compared to baseline ( $\mathrm{p}<0.05$, uncorrected); there was no significant activation to the conditions in the left hemisphere. Only fear and happy survived the small volume correction in the right hemisphere ( $p<0.05$, corrected). See Table 5-12.

$\mathrm{P}$-values of 0.000 indicate a significance level of $\mathrm{p}<0.001$. $*$ Symbolizes significance at the corrected level.

\begin{tabular}{|lcccc|}
\hline $\begin{array}{l}\text { Table 5-12 } \\
\text { Right Sup } \\
\text { Orb }\end{array}$ & $\begin{array}{c}\text { Size of } \\
\text { activation } \\
\text { cluster } \\
\text { (voxels) }\end{array}$ & $\begin{array}{c}\text { Center of activation } \\
\text { (MNI) } \\
\mathbf{x}, \mathbf{y}, \mathbf{z}\end{array}$ & p-value & $\begin{array}{c}\text { Percent of active } \\
\text { voxels in VOI }\end{array}$ \\
Fear & 78 & $16,36,-18$ & $0.001^{*}$ & $7.8 \%$ \\
Surprise & 29 & $16,60,-16$ & 0.009 & $2.9 \%$ \\
Happy & 93 & $16,36,-18$ & $0.000^{*}$ & $9.3 \%$ \\
Control & 57 & $22,34,18$ & 0.002 & $5.7 \%$ \\
\hline
\end{tabular}


e. Superior Frontal Sulcus/Gyrus: Both left and right gyri significantly activated to all conditions compared to baseline (right: $\mathrm{p}<0.001$, uncorrected; left: $\mathrm{p}<0.05$, uncorrected). All four conditions compared to baseline survived small volume correction in the right hemisphere only ( $\mathrm{p}<0.05$, corrected). See Table 5-13.

Shaded areas represent the condition with the highest significant voxel cluster for each volume of interest. P-values of 0.000 indicate a significance level of $p<0.001$. * Symbolizes significance at the corrected level.

\begin{tabular}{|lcccc|}
\hline Table 5-13 & $\begin{array}{c}\text { Size of } \\
\text { activation } \\
\text { cluster } \\
\text { (voxels) }\end{array}$ & $\begin{array}{c}\text { Center of activation } \\
\text { (MNI) }\end{array}$ & $\begin{array}{c}\text { p-value } \\
\mathbf{x}, \mathbf{y}, \mathbf{z}\end{array}$ & $\begin{array}{c}\text { Percent of active } \\
\text { voxels in VOI }\end{array}$ \\
Fear & 8 & $-32,-8,66$ & 0.001 & $0.2 \%$ \\
Surprise & 7 & $-12,4,52$ & 0.002 & $0.2 \%$ \\
Happy & 10 & $-12,4,52$ & 0.002 & $0.3 \%$ \\
Control & 4 & $-12,4,52$ & 0.005 & $0.1 \%$ \\
Right Sup & & $36,-6,58$ & & \\
Fear & 109 & $36,-8,58$ & $0.000^{*}$ & $2.7 \%$ \\
Surprise & 67 & $36,-6,58$ & $0.000^{*}$ & $1.7 \%$ \\
Happy & 37 & $36,-6,58$ & $0.000^{*}$ & $0.9 \%$ \\
Control & 65 & & $0.000^{*}$ & $1.6 \%$ \\
\hline
\end{tabular}




\subsection{3 fMRI Results Summary}

Table 5-14 Significant conditions with the largest voxel cluster for each volume of interest. Shaded entries represent left hemisphere volumes.

\begin{tabular}{|c|c|c|c|}
\hline Fear & Surprise & Happy & Control \\
\hline Left Cingulum & \multirow[t]{11}{*}{ Left Fusiform } & Left Amygdala & Left Temporal \\
\hline Right Cingulum & & Left Fusiform & Left Insula \\
\hline Right Cuneus & & Left Parietal & Right Temporal \\
\hline $\begin{array}{l}\text { Right Middle } \\
\text { Frontal Gyrus }\end{array}$ & & Left Cuneus & Right Insula \\
\hline \multirow[t]{7}{*}{$\begin{array}{l}\text { Right Superior } \\
\text { Frontal Gyrus }\end{array}$} & & $\begin{array}{l}\text { Left Pars } \\
\text { Triangularis }\end{array}$ & Right Parietal \\
\hline & & $\begin{array}{l}\text { Left Pars } \\
\text { Opercularis }\end{array}$ & $\begin{array}{l}\text { Right Pars } \\
\text { Triangularis }\end{array}$ \\
\hline & & $\begin{array}{l}\text { Left Middle Frontal } \\
\text { Gyrus }\end{array}$ & \multirow[t]{5}{*}{$\begin{array}{l}\text { Right Pars } \\
\text { Opercularis }\end{array}$} \\
\hline & & $\begin{array}{l}\text { Left Superior } \\
\text { Frontal Gyrus }\end{array}$ & \\
\hline & & Right Amygdala & \\
\hline & & Right Fusiform & \\
\hline & & $\begin{array}{l}\text { Right Supraorbital } \\
\text { Sulcus }\end{array}$ & \\
\hline
\end{tabular}


Table 5-15 Hemispheric comparison of each total active voxel percentage for each VOI. Shaded entries represent highest values for each VOI.

\begin{tabular}{|l|c|c|c|}
\hline VOI & Left & Right \\
\hline Amygdala & $14.8 \%$ & $4.7 \%$ \\
\hline Fusiform Gyrus & $34.8 \%$ & $41.0 \%$ \\
\hline Temporal & $14.5 \%$ & & $34.3 \%$ \\
\hline Insula & $18.2 \%$ & & $18.4 \%$ \\
\hline Parietal & $46.7 \%$ & & $26.4 \%$ \\
\hline Cingulum & $10.5 \%$ & & $8.8 \%$ \\
\hline Cuneus & $0.8 \%$ & & $80.1 \%$ \\
\hline Par Triangularis & $34.7 \%$ & & $83.3 \%$ \\
\hline Pars Operculum & $63.0 \%$ & & $17.9 \%$ \\
\hline $\begin{array}{l}\text { Frontal Middle } \\
\text { Gyrus }\end{array}$ & $5.2 \%$ & & $9.3 \%$ \\
\hline Surpaorbital Sulcus & None & & $2.7 \%$ \\
\hline $\begin{array}{l}\text { Superior Frontal } \\
\text { Gyrus }\end{array}$ & $0.3 \%$ & & \\
\hline
\end{tabular}

$\square$ Fear

$\square$ Surprise

$\square$ Happy

$\square$ Control 


\subsection{Discussion}

Previous evidence indicates that the amygdala is able to use increases in eye white area (EWA) as a mechanism to detect fear (Whalen et al., 2004); when eye information is the only cue present, the left amygdala is able to distinguish fear from expressions that also produce similar increases in EWA, such as shifts in gaze (Hardee et al., 2008). Surprisingly, the current study indicates that when presented with the eye and eyebrow region of two facial expressions that are identical in EWA increases (specifically, fear and surprise; Figure 5-1), the amygdala does not distinguish between the two despite their difference in eyebrow configuration. Instead, both the left and right amygdala exhibited a greater response to happiness compared to the other conditions despite the fact that this emotion elicits a decrease in EWA (Figure 5-4). During postscan debriefing, subjects reported seeing angry expressions in the task even though no expressions of anger were included in the study. When asked which faces resembled anger, subjects described slightly raised brows and eyes that squinted - which in actuality represented the happiness condition. Based on this, a post-hoc behavioral study was run using naïve subjects and allowed them to freely assign emotions to the eye images from the fMRI task. Happiness was identified correctly only $36 \%$ of the time and when incorrectly identified, it was most often labeled as "angry" or "confused" and therefore was not easily identifiable. If, when viewing only the eye/eyebrow portion of the face, happiness was easily confused for anger, then why would this heighten activation within the amygdala?

The amygdala is known for its role in the detection of fearful faces, but has also been implicated in the processing of angry expressions. Anger signals an increased probability for threat and, like fear, is a negative emotion; Ekman and Friesen (1976) documented no significant differences the ability to identify fearful and angry face stimuli. The perception of both fear and anger have been shown to occur rapidly, automatically, and below the threshold of conscious detection (Morris et al., 1998b; Vuilleumier et al., 2001; Anderson et al., 2003; Whalen et al., 2004); additionally both are perceived more rapidly than non-threatening expressions, such as sadness and happiness (Fox et al., 2000; Öhman et al., 2001) and can elicit phobic responses without the conscious representation of the stimulus (Öhman and Soares, 1993). Furthermore, angry faces are especially adept at garnering attention in human infants (Serrano et al., 1992) and in adults are able to be detected in a crowd much faster than happy faces (Hansen and Hansen, 1988). Additionally, direct gaze serves as an intensifier of emotional expressions (Ellsworth, 
1975) and anger paired with a gaze pointed at the viewer clearly signals the expresser as the source of threat. However, these reasons adequately explain the elevated levels of amygdala activation to happy/angry faces seen here, but do not account for an increased activation to these faces over fear.

One potential explanation lies in the ambiguity of viewing a happy expression using only the eyes; the identification of happiness typically relies on information from the mouth, therefore when only the eyes are visible, happy faces are harder to discern from other emotions where the eyes are also pursed (i.e. anger). LeDoux (1996) hypothesized that when an animal is faced with an ambiguous stimulus (snake or stick), the default perception errs towards that of threat, and further processing would then determine whether danger is imminent. Evolutionarily, this approach ensures a greater chance of survival and extends to human emotions - when an expression is ambiguous in nature, it is initially perceived as the more threatening of all choices (Fox et al., 2006). Thus, if subjects were unsure of the exact emotion portrayed by the eyes but thought it could either be happiness or anger, anger then wins as the more threatening emotion. And while it is true that both surprise and fear were interchangeably identified as one another in our post-hoc behavioral study and could also be labeled as ambiguous, the BOLD signal of the amygdala has been shown to habituate to the repeated presentation of face stimuli (Fischer et al., 2003). Therefore, diminished amygdala activation to fear and surprise compared to happy could reflect the similarities between the fear and surprise stimuli in that they were viewed as the same stimulus type; in this sense this "singular" condition would have been presented twice as many times compared to the happy and neutral conditions across the scanning session and was prone to greater degrees of habituation. Another possible explanation for the increased activation to the happy/anger condition lies in the relevance that emotions play in human socialization. It is likely that angry faces are encountered more often than those expressing fear. In fact, two separate studies have shown that subjects reported seeing a greater number of angry than fearful faces in their lifetime (Bond and Siddle, 1996; Whalen, 1998). Experience with happy and neutral faces were also ranked higher than that of fearful faces, indicating that viewing fear in the face of another is atypical and somewhat novel. Wilson and Rolls (1993) used primate electrophysiology to demonstrate maximum activation of neurons within the amygdala to novel stimuli, which was followed by a decrease in activation with increasing stimulus familiarity. Accordingly, viewing fearful eyes could activate the amygdala due to the novelty of this stimulus, but repeated 
exposure could cause amygdala activation to decrease. Anger, on the other hand, is an emotion more readily encountered and conveys the source of the threat more directly to the viewer. In this case, the amygdala would remain vigilant and activation to anger would not decrease rapidly. This makes sense, in my opinion, from an evolutionary point of view as modern humans live in far different conditions than our early ancestors. Vigilance toward potential dangers in the environment was imperative to survival when living in small groups and primitive conditions. Humans today live in much larger social groups and a variety of surroundings; it could be argued that in present times detecting anger in the face of another is more common and vital than viewing fear. Similarly, amygdala activation to EWA increases could be indicative of the residual importance that fearful expressions once had (and still do, but to a lesser extent).

Effects of laterality in the amygdala were surprisingly different than what was previously found (Chapter 4): the right amygdala showed a general activation to all conditions while the left showed a preference for one condition (fear) but little to no activation for the remaining three conditions. Here, the left amygdala showed a general activation to all conditions - although the response to happiness was far greater compared to the other conditions - while the right showed a response to one condition (happiness) and little to no activation to the remaining three (Table 5-14). Despite this difference, the activation in the left amygdala was far greater than in the right (Table 5-14) and in general, the many VOIs in the left hemisphere also showed a preference to happiness. This combination of activity could reflect the ambiguity of viewing happiness only from the eye regions; if subjects perceived the happy condition as being angry, then anger would be perceived as the more threatening emotion by default and the leftward bias seen in the amygdala and other regions could indicate specificity toward threatening emotions, similar to our previous experiment. In addition, it is thought that the right amygdala responds in a rapid and automatic manner to stimuli that are generally arousing and habituates faster than the left (Glascher and Adolphs, 2003), explaining the lower degree of activity in this VOI. The left amygdala is proposed to activate to stimuli that are emotionally stimulating and have a high level of arousal; this also fits in with our current data as happiness is being viewed as a threat and therefore is seen as more arousing.

Even though the left hemisphere showed a preference for the happy condition, Table 5-15 illustrates a greater percentage of right hemisphere activation across all VOIs. This is in line with evidence supporting a right-hemispheric dominance for face perception (Sergent and Bindra, 
1981; Rhodes, 1985; Luh et al., 1991) and it is very plausible that this extends to face parts as well. In face specific regions such as the fusiform gyrus, we did not see a greater specificity for one emotion over another however this is not surprising as the fusiform is more associated with the extraction facial identity and not emotion recognition per se (Haxby et al., 2000). Temporal regions also showed a greater percentage of right hemisphere activation, specifically in the right posterior superior temporal sulcus (STS). The STS is known for responding to biological motion such as whole moving bodies, hand motion, and eye and mouth movements (Puce et al., 1998; Decety and Grezes, 1999; Calvert and Campbell, 2003; Puce et al., 2003; Wheaton et al., 2004) as well as to still pictures of faces (Kanwisher et al., 1997; Chao et al., 1999; Ishai et al., 2005). Pelphrey et al. (2005) states that eye movements evoke activity in the posterior STS (specifically the right side) which is in line with our results here and prior studies (Puce et al., 1998; Wicker et al., 1998; Hoffman and Haxby, 2000; Pelphrey et al., 2004; Pelphrey et al., 2005). We did not see a specific response to any emotion over another, which is not surprising considering that the STS is mainly responsible for processing the changeable aspects of the face however it is worth noting that if subjects were required to attend to emotion and not to the shape-task, the STS may prefer one emotion over another. It has been shown that instructing subjects to actively search for information within eyes and faces can alter or bias activation in the amygdala (Hooker et al., 2003; Phillips et al., 2004). Moreover, STS activity has been shown to be enhanced for potentially threatening actions (Wheaton et al., 2001) and whole-body expressions of fear (de Gelder et al., 2004), however whether this extends to face parts is not entirely clear.

In summary, this experiment revealed that the amygdala also responds to stimuli that are ambiguous in nature and need to be investigated further. Similar to Experiment 2 (Chapter 4), there was a laterality effect in that the left amygdala responded significantly higher to one condition compared to the other conditions. This suggests that the amygdala is not only involved in detecting fear in the face of another, but also responds to face stimuli that are unclear but signal a possible threat. 


\subsection{Figures}

Figure 5-1 (14)

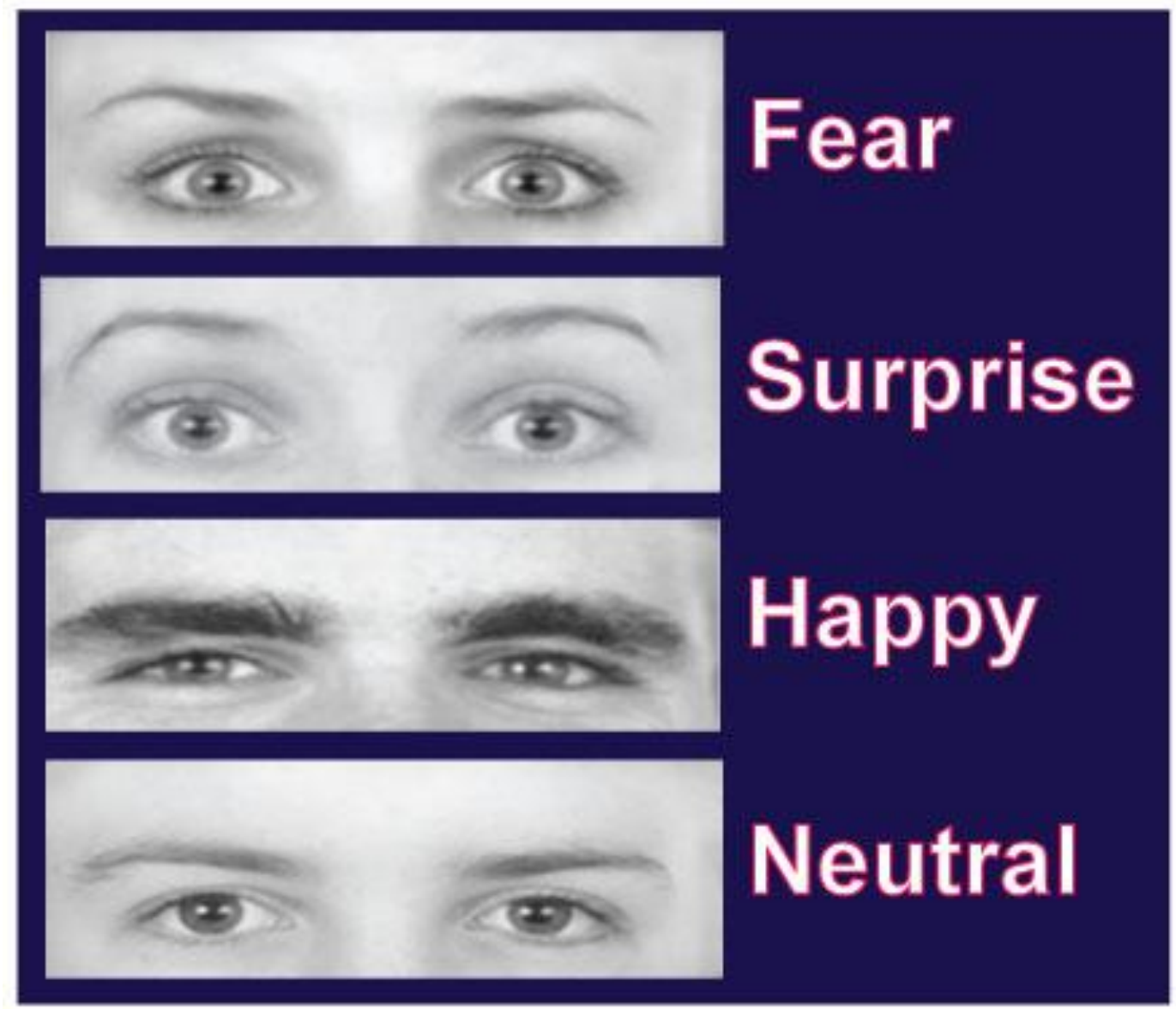

Figure 5-1. Example of stimuli from each condition set. Note how fear and surprise look identical except for the configuration of the eyebrows. 
Figure 5-2 (15)

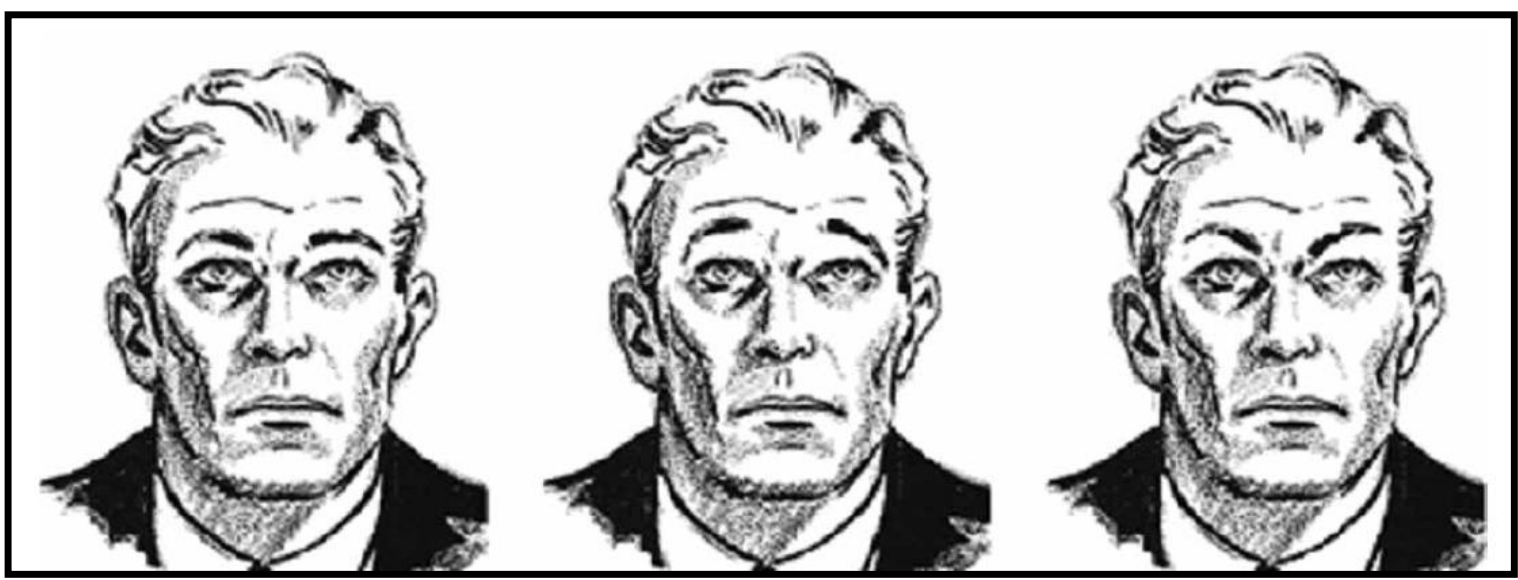

Figure 5-2. These cartoon faces are identical except for their eyebrows. Alone, or in concert with other facial movements, changes in the angle, height, and curvature of the eyebrows can alter the emotional expression of a face and may play an integral role in nonverbal communication. Both figure and figure caption were borrowed from another source (Sadr et al., 2003). 
Figure 5-3 (16)

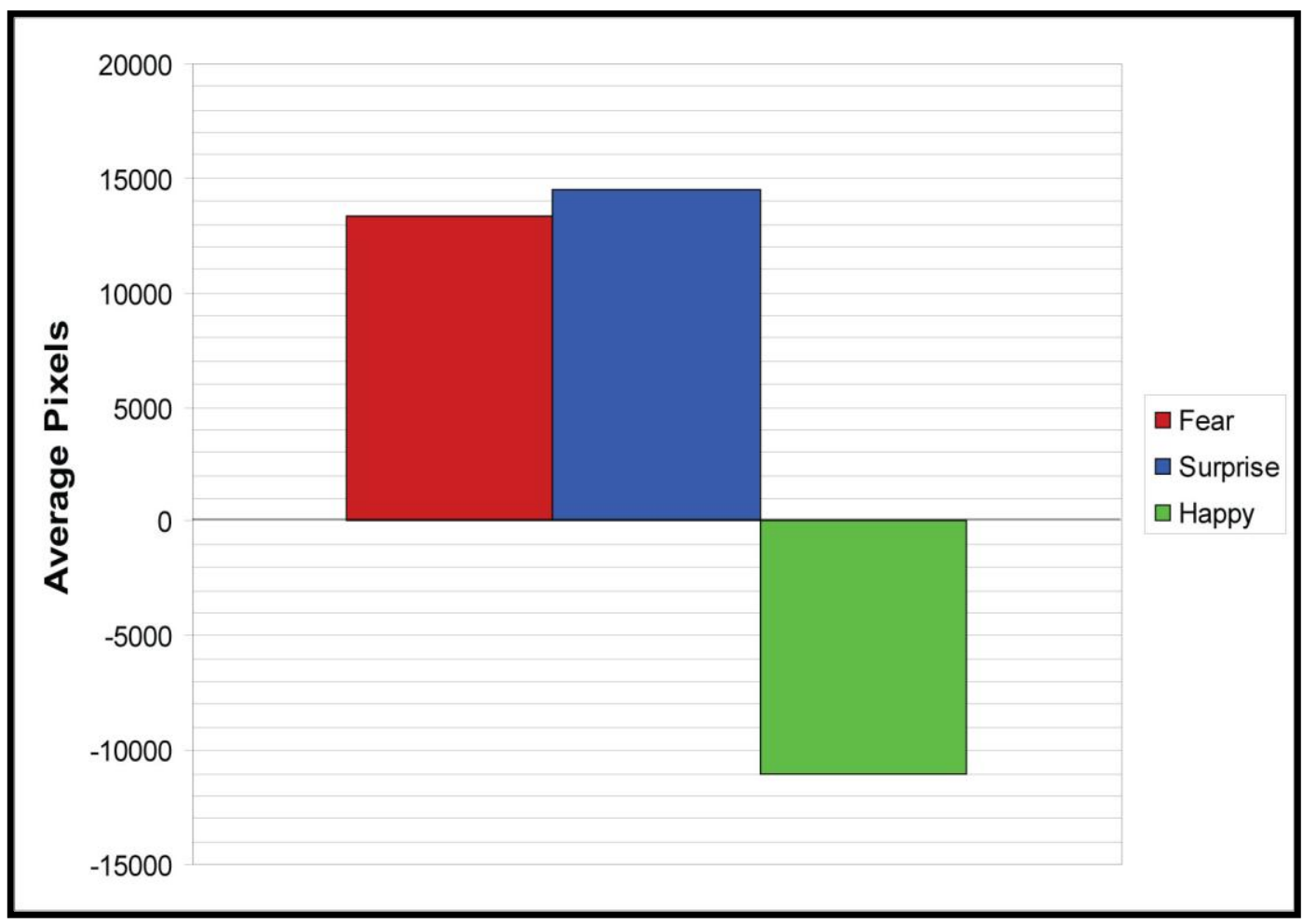

Figure 5-3. Average pixel change for each condition from the matching neutral expression. 
Figure 5-4 (17)
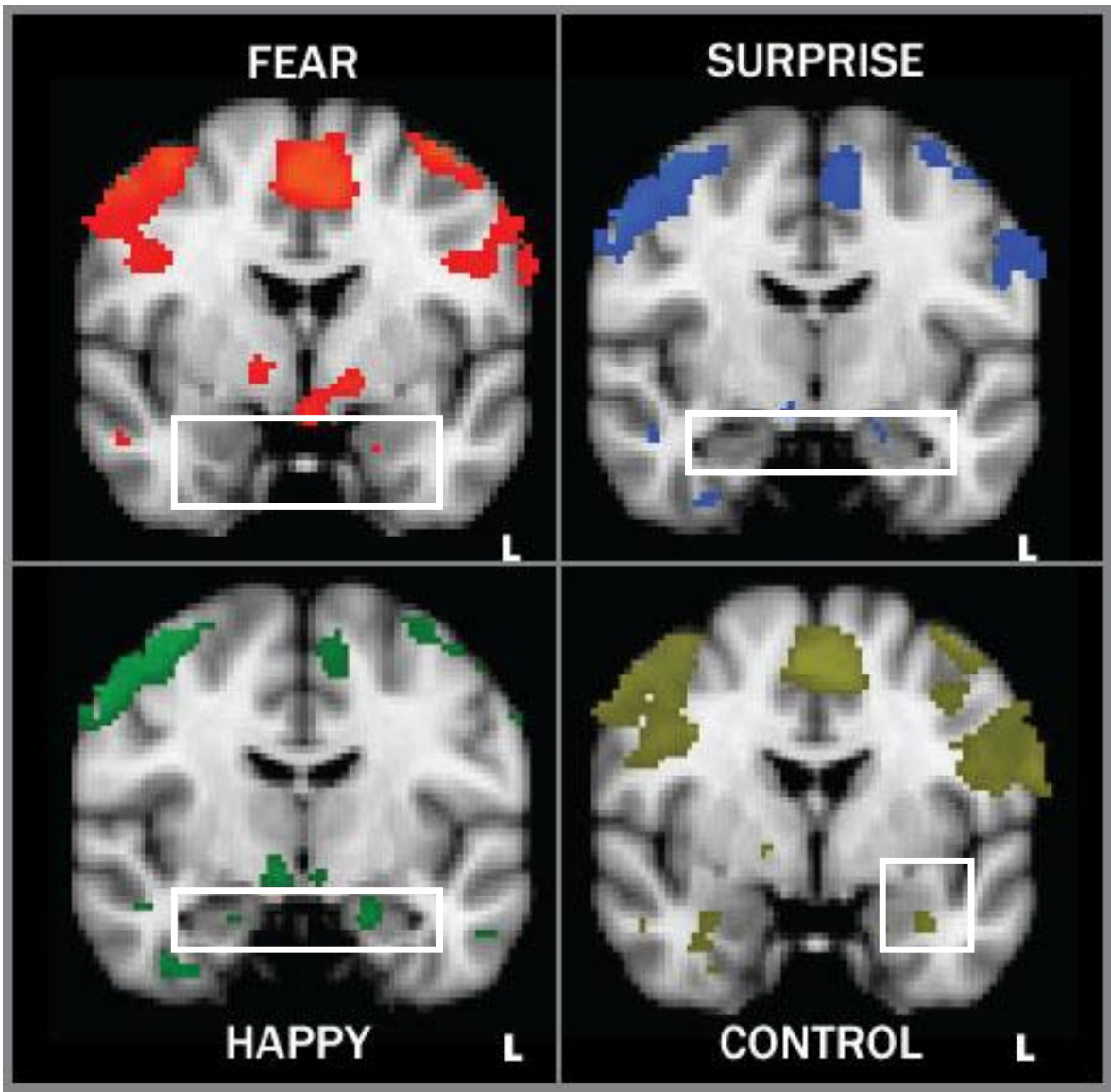

Figure 5-4. BOLD activation in the amygdala (area in box) to each of the emotional conditions averaged across subjects and compared to baseline at $\mathrm{p}<0.05$ uncorrected $(\mathrm{L}=$ left side of the brain). 
Figure 5-5 (18)

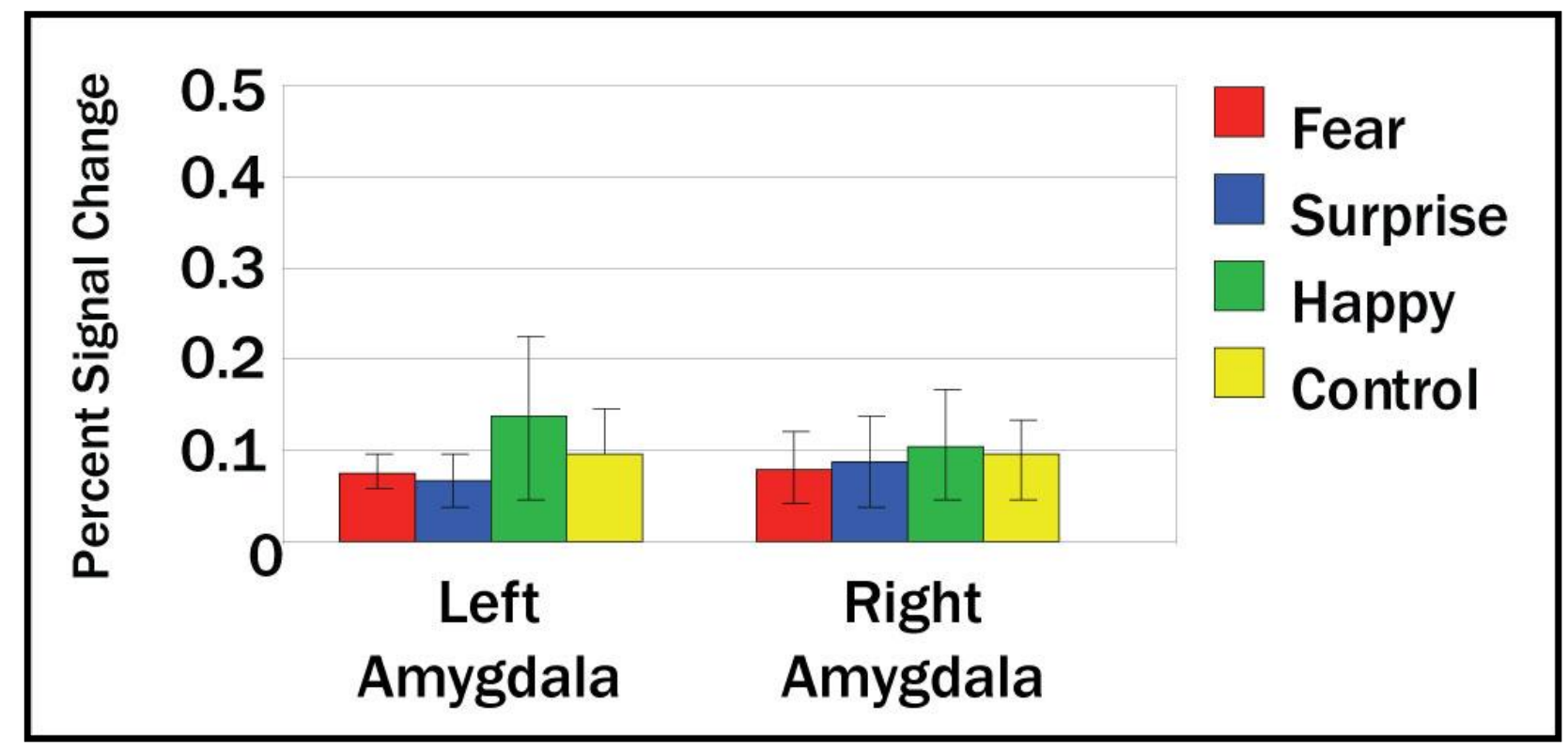

Figure 5-5. Percent signal change for the left and right amygdala. Fear was significantly greater than the other conditions in the left amygdala (signified by *). 
Figure 5-6 (19)

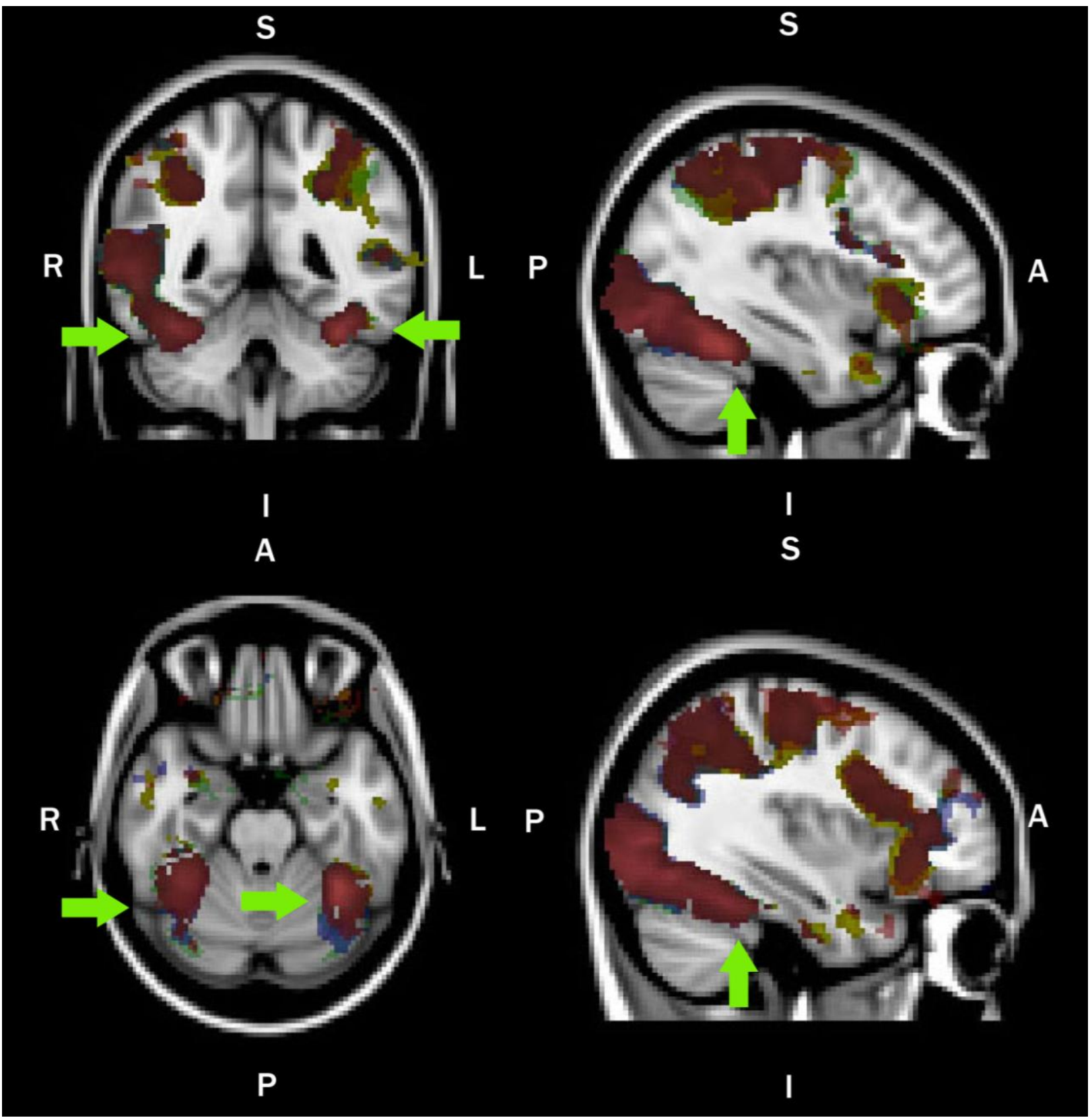

Figure 5-6. Activation to all four conditions at the $p<0.05$ significance level for the fusiform gyrus (green arrows). Fear $=$ red; Surprise $=$ blue; Happy = green; Control = yellow . Areas where color deviates from this scheme indicate overlapping activation for two or more conditions. All four conditions showed similar patterns of activation within the fusiform gyrus. 
Figure 5-7 (20)

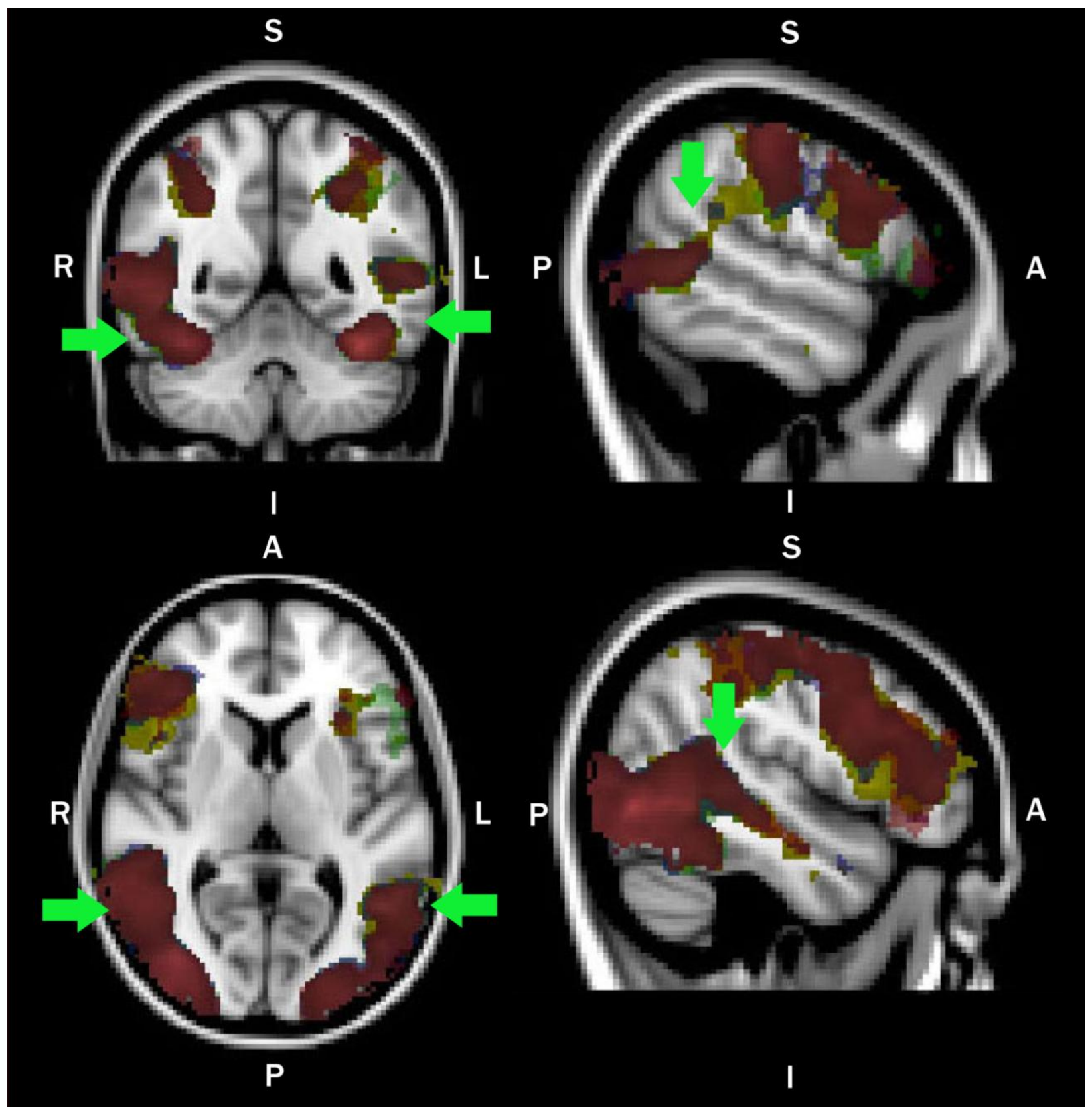

Figure 5-7. Activation to all four conditions at the $p<0.05$ significance level within the temporal VOI. Green arrows highlight areas within this VOI such as the superior temporal sulcus/gyrus and the middle tempral sulcus/gyrus. Fear = red; Surprise = blue; Happy = green; Control $=$ yellow. Areas where color deviates from this scheme indicate overlapping activation for two or more conditions. All four conditions showed similar patterns of activation within the temporal VOI. 
Figure 5-8 (21)

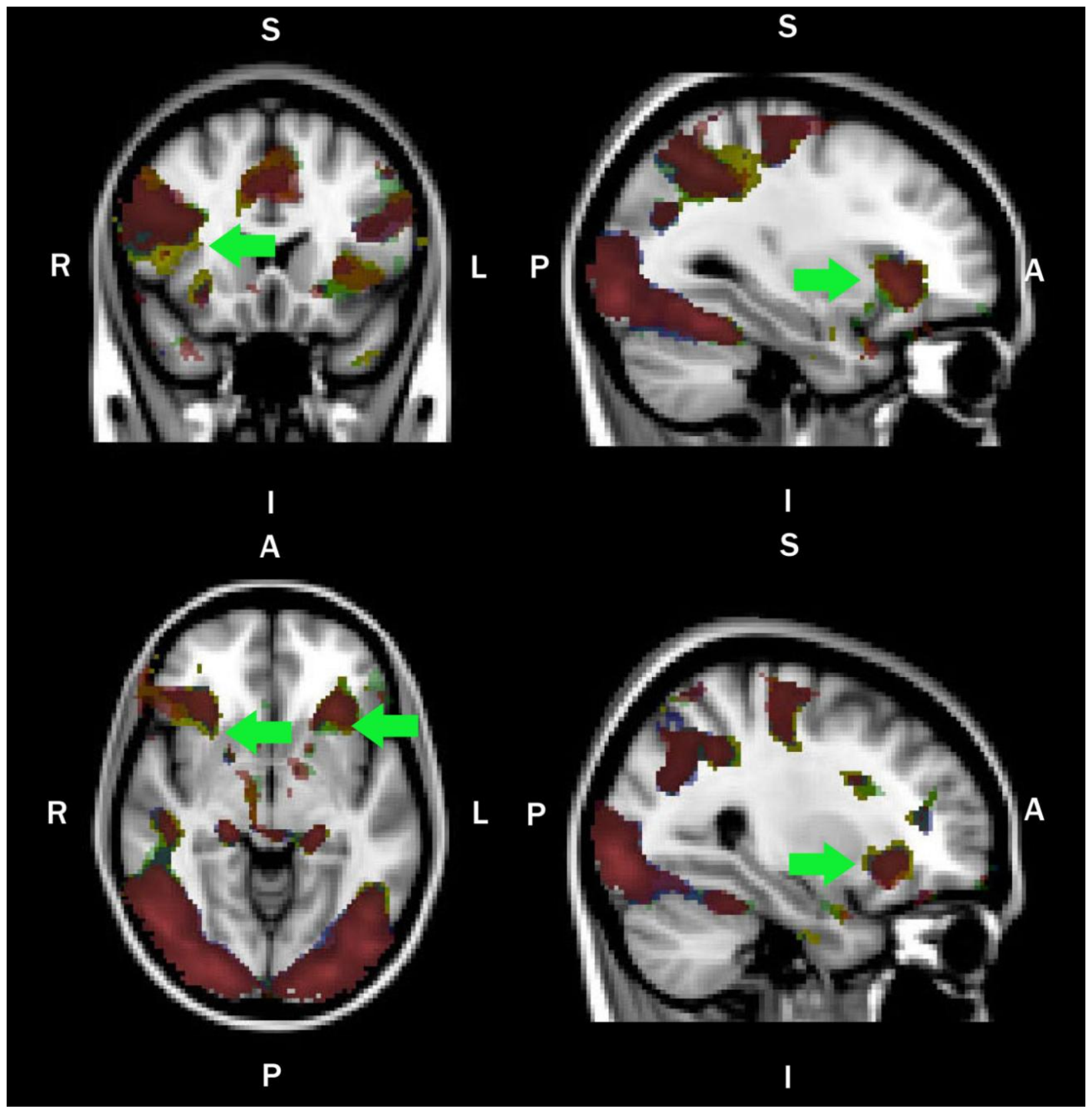

Figure 5-8. Activation to all four conditions at the $p<0.05$ significance level within the insula VOI (arrows). Fear = red; Surprise = blue; Happy = green; Control = yellow. Areas where color deviates from this scheme indicate overlapping activation for two or more conditions. All four conditions showed similar patterns of activation within the insula VOI. 
Figure 5-9 (22)

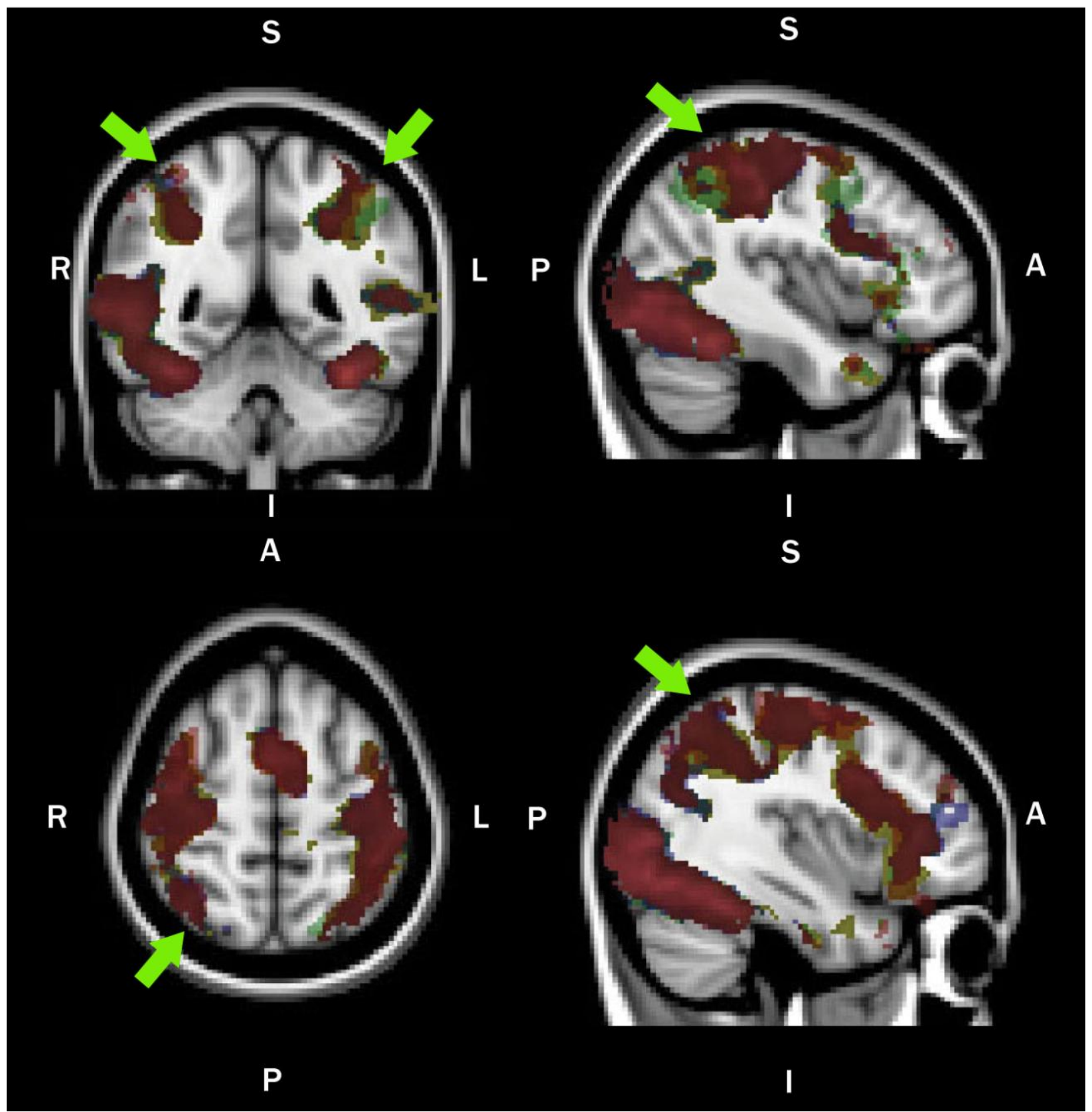

Figure 5-9. Activation to all four conditions at the $p<0.05$ significance level within the parietal VOI, highlighting areas such as the IPS (arrows). Fear = red; Surprise = blue; Happy = green; Control = yellow. Areas where color deviates from this scheme indicate overlapping activation for two or more conditions. All four conditions showed similar patterns of activation within the parietal VOI. 


\section{CHAPTER 6: Experiment 4 - Amygdala Activation to Emotional Mouths}




\subsection{Introduction}

A number of informative visual cues emanate from the human face, including age, gender, mental state, and intentions. Of all facial features, the eyes are particularly adept at transmitting social and emotional information to others. As addressed earlier (Chapters 4 and 5), the eye region of fearful faces has been shown to activate the amygdala due to increases in the visible amount of sclera, or eye white area (EWA), which is an extremely salient cue that can be viewed up close, at a distance, and with or without the presence of fine facial detail. However, less is known about what role another prominent and dynamic facial feature, the mouth, plays in the detection and recognition of fear, especially when other facial information is not available.

The mouth, like the eyes, is a source for a wealth of social information: the quintessential characteristic of happiness is a smile, while anger manifests as a tightened mouth with pursed lips (Smith et al., 2005) illustrating how the mouth plays a vital role for the identification of certain emotions. Prosopagnosic patients develop a more robust representation of the mouth as it may contain more information for them compared to any single element from the upper face (Orban de Xivry et al., 2008), which is a useful strategy for these individuals to recognize personally familiar faces (Caldara et al., 2005). In normal subjects, the mouth is pivotal for distinguishing happiness from other emotions due to its marked perceptibility and ability to easily attract attention (Calvo and Nummenmaa, 2008). Conversely, a fearful mouth lacks the obvious saliency that emanates from a smile or even in the wide-eyed look of fear yet, if the ability to perceive fear in another's face is fundamental for survival, the ability to recognize fear from more than one facial cue would be exceptionally useful. Furthermore, instances occur when another's face is only partially visible - due to wearing sunglasses or simply from an obstruction, for example - and therefore it is unlikely that the discernment of fear relies only on the eyes.

It is possible that the amygdala does recognize cues from a fearful mouth despite it lacking any obvious elements that would attract visual attention. It has long been hypothesized that two parallel pathways exist in visual processing, the first being a coarsely-tuned, subcortical route and the second a more fine-tuned, cortical path allowing for the processing of greater detail (for a review, see Adolphs and Spezio, 2006). The subcortical pathway includes structures such as the superior colliculus, the pulvinar, and the amygdala, and would allow for the rapid detection of conspicuous stimuli that are potentially threatening, such as fearful eye whites (see Chapter 4). The cortical pathway, including such structures as the STS and fusiform gyrus, 
would process information in a more detailed manner and thus could take into consideration features that are not exceptionally salient, such as the downturned corners of the mouth. This pathway could then communicate with the amygdala to modulate activity appropriately depending on whether the stimulus represented a true threat or not. Based on the idea that the amygdala is a player in both subcortical and cortical visual processing streams and that it is known to preferentially activate to fearful eye configurations, it is my hypothesis that the amygdala will preferentially activate to the fearful mouth compared to that of other emotions. 


\subsection{Methods}

Prior to the fMRI study, stimuli were tested using a behavioral experiment in order to ascertain if each emotion could be identified using only the mouth region of the face. Two different sets of subjects were used in each experiment so there was no subject overlap between the two studies.

\subsubsection{Participants}

6.2.1a Behavioral study: Fourteen neurologically normal subjects ( 8 females, 12 right-handed, aged 23-31 years of age) consented to participate in a study approved by the Institutional Review Board of West Virginia University. All subjects had either normal or corrected-to-normal vision.

6.2.1b fMRI study: Thirteen neurologically normal subjects (4 female, 11 right-handed, aged 2032 years of age) consented to participate in a study approved by the Institutional Review Board of West Virginia University. All subjects had either normal or corrected-to-normal vision.

\subsubsection{Stimuli}

Stimuli were selected from the JACFEE/JACNeuF series of faces by Ekman and Matusomoto (Paul Ekman Group LLC, Berkeley, CA, USA) and could belong to one of four categories (conditions): fear, surprise, happy, and motion control. Stimuli were created using the exact same parameters outlined in Section 5.3.2 except that stimuli consisted of the mouth region of faces instead of the eyes. For an example of these stimuli, see Figure 6-1.

\subsubsection{Task design}

The task design was identical to that outlined in Section 5.3.3 except that mouth stimuli were used instead of eye stimuli. The behavioral study followed the methods outlined in Section 5.3.3a and the fMRI study followed the methods outline in Section 5.3.3b

\subsubsection{Imaging procedure and analysis}

Imaging procedures and analysis followed the methods detailed in Section 5.3.4. 


\subsection{Results}

\subsubsection{Behavioral Study}

Percent accuracy on the emotion identification task varied as a function of condition only for the conditions of fear vs. surprise (fear > happy: $F[1,27]=11.8, p=0.001957$; happy > surprise: $F[1,27]=24.5, p=0.000034)$. On incorrect trials, fear was most often identified as no emotion (13.5\%), happy was most often identified as surprise (1\%), surprise was most often identified as fear (4\%). Reaction time (ms) did vary as a function of condition (fear $>$ surprise $\mathrm{F}[1,27]=5.11, \mathrm{p}=0.032004$; happy $>$ surprise $\mathrm{F}[1,27]=17.7, \mathrm{p}=0.000251$ ).

\begin{tabular}{|l|c|c|}
\hline Table 6-1 & $\begin{array}{c}\text { Average Percent Correct for } \\
\text { Behavioral Subjects }(\mathbf{n}=\mathbf{1 4})\end{array}$ & $\begin{array}{c}\text { Average Correct Response } \\
\text { Time }(\mathbf{m s})\end{array}$ \\
\hline Fear & $81.2 \pm 23.3$ & $775.5 \pm 317.1$ \\
\hline Surprise & $74.6 \pm 24.2$ & $851.6 \pm 327.0$ \\
\hline Happy & $96.7 \pm 4.7$ & $721.8 \pm 256.1$ \\
\hline
\end{tabular}

\subsection{2 fMRI Study}

6.3.2a Behavioral data: Percent accuracy on the shape identification task did not differ as a function of shape (mean sd: circle $=97.6 \pm 2.5 ;$ square $=97.7 \pm 3.8 ; \mathrm{F}[1,12]=0.20, \mathrm{p}=$ 0.890783). Reaction time (ms) also did not vary as a function of condition (mean sd: circle $=$ $577.1 \pm 129.0 ;$ square $=579.1 \pm 119.6 ; \mathrm{F}[1,12]=0.06, \mathrm{p}=0.816004)$.

Post-scan debriefing of subjects revealed that all subjects reported seeing happiness or smiling in the mouth images, 7 subjects reported that the mouths were frowning. No subject reported seeing surprise or fear in the images. 


\subsection{2b fMRI data}

The data presented in this section is broken up into 2 sections: primary and secondary data. The primary data section contains the VOIs that are considered to be core to face/emotion processing and/or show the same pattern of activation as the amygdala (the FG, the STS, the IPS, and the insula). The secondary data section contains VOIs that have been shown to be involved in face/emotion processing but with less consistency.

The following tables display size of activation clusters (in voxels), the MNI coordinates for these centers of activation, and the p-values for each VOI. P-values of 0.000 indicate a significance level of $\mathrm{p}<0.001$. Symbolizes significance at the corrected level. Percent of activation cluster size represents the number of active voxels per total number of voxels in the VOI. 


\subsection{2b-1 Primary Data}

Amygdala: The BOLD response to each of the four mouth conditions was analyzed to examine amygdala activation to EWA differences. Of the four conditions, only the happy condition showed any significant activation (compared to baseline) in the right amygdala while all four conditions elicited left amygdala activation. See Table 6-2. For a visual depiction of activation within the amygdala ROI, see Figure 6-2. A chart of the percent signal change in the amygdala from baseline can be seen in Figure 6-3.

P-values of 0.000 indicate a significance level of $\mathrm{p}<0.001$. $*$ Symbolizes significance at the corrected level.

\begin{tabular}{|c|c|c|c|c|c|}
\hline \multicolumn{6}{|l|}{ Table 6-4 } \\
\hline $\begin{array}{l}\text { Left } \\
\text { Amygdala }\end{array}$ & $\begin{array}{c}\text { Size of } \\
\text { activation } \\
\text { cluster } \\
\text { (voxels) }\end{array}$ & $\begin{array}{c}\text { Center of } \\
\text { activation } \\
(\mathrm{MNI}) \\
(\mathbf{x}, \mathbf{y}, \mathbf{z})\end{array}$ & p-value & $\begin{array}{c}\text { Percent of } \\
\text { active voxels } \\
\text { in VOI }\end{array}$ & $\begin{array}{c}\text { Percent } \\
\text { signal } \\
\text { change }\end{array}$ \\
\hline Fear & 1 & $-20,-8,-20$ & 0.050 & $0.4 \%$ & $0.069 \%$ \\
\hline Surprise & 1 & $-20,-8,-20$ & 0.038 & $0.4 \%$ & $0.083 \%$ \\
\hline Happy & 3 & $-20,-8,-20$ & 0.010 & $1.2 \%$ & $0.099 \%$ \\
\hline Control & 7 & $-24,-2,-26$ & 0.009 & $2.8 \%$ & $0.121 \%$ \\
\hline \multicolumn{6}{|l|}{$\begin{array}{l}\text { Right } \\
\text { Amygdala }\end{array}$} \\
\hline Happy & 2 & $22,-6,-18$ & 0.037 & $0.8 \%$ & $0.113 \%$ \\
\hline $\begin{array}{l}\text { Fear > } \\
\text { Control }\end{array}$ & 22 & $18,-4,-26$ & 0.010 & $8.6 \%$ & not available \\
\hline $\begin{array}{l}\text { Surprise > } \\
\text { Control }\end{array}$ & 3 & $14,-2,-18$ & 0.034 & $1.2 \%$ & not available \\
\hline $\begin{array}{l}\text { Fear > } \\
\text { Surprise }\end{array}$ & 1 & $20,0,-28$ & 0.050 & $0.4 \%$ & not available \\
\hline
\end{tabular}


Fusiform Gyrus: Both the left and right fusiform gyri activated to all four conditions compared to baseline ( $\mathrm{p}<0.001$, uncorrected). F. When small volume corrections were applied, all four conditions (fear, surprise, happy, control) versus baseline passed in both the left and right fusiform $(\mathrm{p}<0.05$, corrected). See Table 6-3. For a visual representation of activation in the fusiform, see Figure 6-4.

P-values of 0.000 indicate a significance level of $\mathrm{p}<0.001$. $*$ Symbolizes significance at the corrected level.

\begin{tabular}{|l|c|c|c|c|c|}
\hline Table 6-3 & $\begin{array}{c}\text { Size of } \\
\text { activation } \\
\text { cluster } \\
\text { (voxels) } \\
\text { Fusiform }\end{array}$ & $\begin{array}{c}\text { Center of } \\
\text { activation } \\
\text { (MNI) } \\
(\mathbf{x}, \mathbf{y}, \mathbf{z})\end{array}$ & p-value & $\begin{array}{c}\text { Percent of } \\
\text { active voxels } \\
\text { in VOI }\end{array}$ & $\begin{array}{c}\text { Percent } \\
\text { signal } \\
\text { change }\end{array}$ \\
\hline Fear & 932 & $-38,-56,-22$ & $0.000^{*}$ & $37.0 \%$ & $0.773 \%$ \\
\hline Surprise & 940 & $-38,-56,-22$ & $0.000^{*}$ & $37.3 \%$ & $0.797 \%$ \\
\hline Happy & 1003 & $-38,-56,-22$ & $0.000^{*}$ & $39.8 \%$ & $0.801 \%$ \\
\hline Control & 937 & $-38,-56,-22$ & $0.000^{*}$ & $37.2 \%$ & $0.705 \%$ \\
\hline $\begin{array}{l}\text { Right } \\
\text { Fusiform }\end{array}$ & 742 & $40,-44,-22$ & $0.000^{*}$ & $32.1 \%$ & $0.888 \%$ \\
\hline Fear & 777 & $40,-44,-22$ & $0.000^{*}$ & $33.6 \%$ & $0.896 \%$ \\
\hline Surprise & 830 & $40,-44,-22$ & $0.000^{*}$ & $35.9 \%$ & $0.919 \%$ \\
\hline Happy & $70,-44,-22$ & $0.000^{*}$ & $34.1 \%$ & $0.771 \%$ \\
\hline Control & & & & \\
\hline
\end{tabular}


Temporal Regions (including STS): Both left and right temporal regions activated to all conditions compared to baseline ( $\mathrm{p}<0.001$, uncorrected). When small volume corrections were applied, all four conditions (fear, surprise, happy, motion control) versus baseline passed in both the left and right temporal regions ( $p<0.05$, corrected). See Table 6-4. For a visual representation of activation in temporal regions, see Figure 6-5.

P-values of 0.000 indicate a significance level of $p<0.001$. $*$ Symbolizes significance at the corrected level.

\begin{tabular}{|c|c|c|c|c|c|}
\hline \multicolumn{6}{|l|}{ Table 6-4 } \\
\hline $\begin{array}{l}\text { Left } \\
\text { Temporal }\end{array}$ & $\begin{array}{c}\text { Size of } \\
\text { activation } \\
\text { cluster } \\
\text { (voxels) }\end{array}$ & $\begin{array}{c}\text { Center of } \\
\text { activation } \\
(\mathrm{MNI}) \\
(\mathbf{x}, \mathbf{y}, \mathbf{z})\end{array}$ & p-value & $\begin{array}{l}\text { Percent of } \\
\text { active voxels } \\
\text { in VOI }\end{array}$ & $\begin{array}{c}\text { Percent } \\
\text { signal } \\
\text { change }\end{array}$ \\
\hline Fear & 1211 & $-44,-46,-26$ & $0.000^{*}$ & $11.6 \%$ & $0.497 \%$ \\
\hline Surprise & 1119 & $-44,-46,-26$ & $0.000^{*}$ & $10.7 \%$ & $0.500 \%$ \\
\hline Happy & 1009 & $-44,-46,-26$ & $0.000^{*}$ & $9.7 \%$ & $0.459 \%$ \\
\hline Control & 909 & $-44,-46,-26$ & $0.000^{*}$ & $8.7 \%$ & $0.475 \%$ \\
\hline \multicolumn{6}{|l|}{$\begin{array}{l}\text { Right } \\
\text { Temporal }\end{array}$} \\
\hline Fear & 3317 & $44,-44,-20$ & $0.000^{*}$ & $29.9 \%$ & $0.694 \%$ \\
\hline Surprise & 2912 & $46,-74,-10$ & $0.000^{*}$ & $26.2 \%$ & $0.757 \%$ \\
\hline Happy & 2809 & $44,-44,-20$ & $0.000^{*}$ & $25.3 \%$ & $0.696 \%$ \\
\hline Control & 3125 & $46,-74,-10$ & $0.000 *$ & $28.1 \%$ & $0.630 \%$ \\
\hline
\end{tabular}


Insula: Both the left and right insula significantly activated to all conditions compared to baseline, with the exception of happy in the right ( $\mathrm{p}<0.005$, uncorrected). When small volume corrections were applied, fear and motion control passed in the right fusiform, but only surprise passed in the left $(\mathrm{p}<0.05$, corrected). See Table 6-5. For a visual representation of activation in the insula, see Figure 6-6.

P-values of 0.000 indicate a significance level of $\mathrm{p}<0.001$. $*$ Symbolizes significance at the corrected level.

\begin{tabular}{|c|c|c|c|c|c|}
\hline \multicolumn{6}{|l|}{ Table 6-5 } \\
\hline Left Insula & $\begin{array}{c}\text { Size of } \\
\text { activation } \\
\text { cluster } \\
\text { (voxels) }\end{array}$ & $\begin{array}{c}\text { Center of } \\
\text { activation } \\
(\mathrm{MNI}) \\
(\mathbf{x}, \mathbf{y}, \mathbf{z})\end{array}$ & p-value & $\begin{array}{c}\text { Percent of } \\
\text { active voxels } \\
\text { in VOI }\end{array}$ & $\begin{array}{c}\text { Percent } \\
\text { signal } \\
\text { change }\end{array}$ \\
\hline Fear & 235 & $-32,26,0$ & 0.000 & $12.6 \%$ & $0.180 \%$ \\
\hline Surprise & 199 & $-34,14,14$ & $0.000 *$ & $10.7 \%$ & $0.141 \%$ \\
\hline Happy & 90 & $-30,20,-2$ & 0.005 & $4.8 \%$ & $0.114 \%$ \\
\hline Control & 119 & $-38,16,10$ & 0.005 & $6.4 \%$ & $0.109 \%$ \\
\hline \multicolumn{6}{|l|}{ Right Insula } \\
\hline Fear & 251 & $38,22,-2$ & $0.000 *$ & $14.2 \%$ & $0.173 \%$ \\
\hline Surprise & 46 & $32,24,0$ & 0.003 & $2.6 \%$ & $0.107 \%$ \\
\hline Happy & no sig. & no sig. & no sig. & no sig. & $0.064 \%$ \\
\hline Control & 186 & $40,24,2$ & $0.000^{*}$ & $10.5 \%$ & $0.166 \%$ \\
\hline
\end{tabular}


Parietal Regions (including IPS): Both the left and right parietal regions significantly activated to all conditions compared to baseline, with the exception of happy in the right ( $\mathrm{p}<0.001$, uncorrected). When small volume corrections were applied, all four conditions (fear, surprise, happy, motion control) versus baseline passed in both the left and right parietal regions $(\mathrm{p}<0.05$, corrected). See Table 6-6. For a visual representation of activation in parietal regions, see Figure 6-7.

P-values of 0.000 indicate a significance level of $\mathrm{p}<0.001 . *$ Symbolizes significance at the corrected level.

\begin{tabular}{|l|c|c|c|c|c|}
\hline Table 6-6 & $\begin{array}{c}\text { Size of } \\
\text { activation } \\
\text { cluster } \\
\text { (voxels) }\end{array}$ & $\begin{array}{c}\text { Center of } \\
\text { activation } \\
\text { (MNI) } \\
\mathbf{( x , y , z )}\end{array}$ & p-value & $\begin{array}{c}\text { Percent of } \\
\text { active voxels } \\
\text { in VOI }\end{array}$ & $\begin{array}{c}\text { Percent } \\
\text { signal } \\
\text { change }\end{array}$ \\
\hline Fear & 2314 & $-26,-58,38$ & $0.000^{*}$ & $51.3 \%$ & $0.457 \%$ \\
\hline Surprise & 2517 & $-26,-58,38$ & $0.000^{*}$ & $55.8 \%$ & $0.431 \%$ \\
\hline Happy & 2856 & $-26,-58,38$ & $0.000^{*}$ & $63.3 \%$ & $0.500 \%$ \\
\hline Control & 2199 & $-54,-36,44$ & $0.000 *$ & $48.7 \%$ & $0.463 \%$ \\
\hline Right Parietal & & & & & \\
\hline Fear & 981 & $34,-52,40$ & $0.000 *$ & $27.5 \%$ & $0.494 \%$ \\
\hline Surprise & 832 & $36,-54,40$ & $0.000 *$ & $23.3 \%$ & $0.428 \%$ \\
\hline Happy & 947 & $32,-48,40$ & $0.000 *$ & $26.5 \%$ & $0.341 \%$ \\
\hline Control & 1065 & $36,-48,40$ & $0.000 *$ & $29.9 \%$ & $0.540 \%$ \\
\hline
\end{tabular}




\subsection{2b-2 Secondary Data}

Cingulum: Both the left and right cingulum significantly activated to all conditions compared to baseline ( $\mathrm{p}<0.001$, uncorrected). When small volume corrections were applied, all four conditions (fear, surprise, happy, motion control) versus baseline passed in the left hemisphere, only happy passed in the right ( $\mathrm{p}<0.05$, corrected). See Table 6-7.

$\mathrm{P}$-values of 0.000 indicate a significance level of $\mathrm{p}<0.001$. $*$ Symbolizes significance at the corrected level.

\begin{tabular}{|c|c|c|c|c|}
\hline \multicolumn{5}{|l|}{ Table 6-7 } \\
\hline $\begin{array}{l}\text { Left } \\
\text { Cingulum }\end{array}$ & $\begin{array}{c}\text { Size of } \\
\text { activation } \\
\text { cluster } \\
\text { (voxels) }\end{array}$ & $\begin{array}{c}\text { Center of } \\
\text { activation (MNI } \\
\mathbf{x}, \mathbf{y}, \mathbf{z}\end{array}$ & p-value & $\begin{array}{c}\text { Percent of } \\
\text { active voxels in } \\
\text { VOI }\end{array}$ \\
\hline Fear & 722 & $-4,8,42$ & $0.000 *$ & $19.0 \%$ \\
\hline Surprise & 454 & $-6,8,42$ & $0.000 *$ & $11.9 \%$ \\
\hline Hарру & 619 & $-6,8,42$ & $0.000 *$ & $16.3 \%$ \\
\hline Control & 503 & $-4,6,42$ & $0.000 *$ & $13.2 \%$ \\
\hline \multicolumn{5}{|l|}{$\begin{array}{l}\text { Right } \\
\text { Cingulum }\end{array}$} \\
\hline Fear & 385 & $2,8,44$ & 0.000 & $10.0 \%$ \\
\hline Surprise & 241 & $8,2,44$ & 0.000 & $6.3 \%$ \\
\hline Нарру & 460 & $8,-4,42$ & $0.000 *$ & $11.9 \%$ \\
\hline Control & 181 & $10,6,42$ & 0.000 & $4.7 \%$ \\
\hline
\end{tabular}


Cuneus: Both the left and right cuneus significantly activated to all conditions compared to baseline, with the exception of happy in the right ( $\mathrm{p}<0.05$, uncorrected). When small volume corrections were applied, only the happy condition passed in the left hemisphere $(\mathrm{p}<0.05$, corrected). See Table 6-8.

$\mathrm{P}$-values of 0.000 indicate a significance level of $\mathrm{p}<0.001$. $*$ Symbolizes significance at the corrected level.

\begin{tabular}{|c|c|c|c|c|}
\hline \multicolumn{5}{|l|}{ Table 6-8 } \\
\hline Left Cuneus & $\begin{array}{c}\text { Size of } \\
\text { activation } \\
\text { cluster } \\
\text { (voxels) }\end{array}$ & $\begin{array}{c}\text { Center of } \\
\text { activation (MNI } \\
\mathbf{x}, \mathbf{y}, \mathbf{z}\end{array}$ & p-value & $\begin{array}{c}\text { Percent of } \\
\text { active voxels in } \\
\text { VOI }\end{array}$ \\
\hline Fear & 45 & $-16,-36,68$ & 0.006 & $0.9 \%$ \\
\hline Surprise & 119 & $-18,-68,60$ & 0.001 & $2.4 \%$ \\
\hline Happy & 249 & $-12,-54,44$ & $0.000 *$ & $4.9 \%$ \\
\hline Control & 11 & $-18,-68,60$ & 0.004 & $0.2 \%$ \\
\hline \multicolumn{5}{|c|}{ Right Cuneus } \\
\hline Fear & 42 & $20,-58,38$ & 0.005 & $0.9 \%$ \\
\hline Surprise & 51 & $20,-98,8$ & 0.005 & $1.1 \%$ \\
\hline Happy & 103 & $20,-60,36$ & 0.001 & $2.2 \%$ \\
\hline Control & 43 & $18,-100,6$ & 0.008 & $0.9 \%$ \\
\hline
\end{tabular}




\section{Frontal Regions}

a. Pars Triangularis: Both left and right pars triangularis significantly activated to all conditions compared to baseline ( $\mathrm{p}<0.001$, uncorrected). All conditions compared to baseline survived small volume correction in both the left and right pars triangularis, with the exception of control in the left ( $p<0.05$, corrected). See Table 6-9.

P-values of 0.000 indicate a significance level of $\mathrm{p}<0.001$. $*$ Symbolizes significance at the corrected level.

\begin{tabular}{|lcccc|}
\hline $\begin{array}{l}\text { Table 6-9 } \\
\text { Left Pars }\end{array}$ & $\begin{array}{c}\text { Size of } \\
\text { activation } \\
\text { cluster } \\
\text { (voxels) }\end{array}$ & $\begin{array}{c}\text { Center of } \\
\text { activation (MNI } \\
\mathbf{x}, \mathbf{y}, \mathbf{z}\end{array}$ & p-value & $\begin{array}{c}\text { Percent of } \\
\text { active voxels in } \\
\text { VOI }\end{array}$ \\
Fear & 741 & $-36,10,24$ & $0.000^{*}$ & $29.3 \%$ \\
Surprise & 965 & $-58,26,24$ & $0.000^{*}$ & $38.2 \%$ \\
Happy & 584 & $-36,10,24$ & $0.000^{*}$ & $23.1 \%$ \\
Control & 443 & $-56,18,16$ & 0.001 & $17.5 \%$ \\
Right Pars & & $48,14,24$ & & $49.1 \%$ \\
Tri & 1056 & $48,14,24$ & $0.000^{*}$ & $39.0 \%$ \\
Fear & 838 & $48,14,24$ & $0.000^{*}$ & $27.9 \%$ \\
Surprise & 600 & $48,14,24$ & $0.000^{*}$ & $53.7 \%$ \\
Happy & 1155 & $0.000^{*}$ & \\
Control & & & & \\
\hline
\end{tabular}


b. Middle Frontal Gyrus: Both left and right gyri significantly activated to all conditions compared to baseline ( $\mathrm{p}<0.05$, uncorrected). All conditions compared to baseline survived small volume correction in the right hemisphere, no conditions survived in the left hemisphere $(\mathrm{p}<$ 0.05, corrected). See Table 6-10.

P-values of 0.000 indicate a significance level of $\mathrm{p}<0.001$. $*$ Symbolizes significance at the corrected level.

\begin{tabular}{|c|c|c|c|c|}
\hline \multicolumn{5}{|c|}{\begin{tabular}{|l|} 
Table 6-10 \\
\end{tabular}} \\
\hline Left Mid & $\begin{array}{c}\text { Size of } \\
\text { activation } \\
\text { cluster } \\
\text { (voxels) }\end{array}$ & $\begin{array}{c}\text { Center of } \\
\text { activation (MNI } \\
\mathbf{x}, \mathbf{y}, \mathbf{z}\end{array}$ & p-value & $\begin{array}{c}\text { Percent of } \\
\text { active voxels in } \\
\text { VOI }\end{array}$ \\
\hline Fear & 109 & $-42,4,56$ & 0.001 & $2.2 \%$ \\
\hline Surprise & 37 & $-46,4,52$ & 0.012 & $0.8 \%$ \\
\hline Нарру & 151 & $-42,4,56$ & 0.001 & $3.1 \%$ \\
\hline Control & 63 & $-40,46,32$ & 0.008 & $1.3 \%$ \\
\hline \multicolumn{5}{|l|}{ Right Mid } \\
\hline Fear & 1146 & $44,-4,56$ & $0.000 *$ & $22.5 \%$ \\
\hline Surprise & 719 & $40,-6,60$ & $0.000 *$ & $14.1 \%$ \\
\hline Hарру & 628 & $48,12,50$ & $0.000 *$ & $12.3 \%$ \\
\hline Control & 929 & $48,14,50$ & $0.000 *$ & $18.2 \%$ \\
\hline
\end{tabular}


c. Superior Frontal Sulcus/Gyrus: Both left and right significantly activated to all conditions compared to baseline (right: $\mathrm{p}<0.001$, uncorrected; left: $\mathrm{p}<0.05$, uncorrected). All four conditions compared to baseline survived small volume correction in the right hemisphere only ( $\mathrm{p}<0.05$, corrected). See Table 6-11.

P-values of 0.000 indicate a significance level of $p<0.001$. $*$ Symbolizes significance at the corrected level.

\begin{tabular}{|c|c|c|c|c|}
\hline \multicolumn{5}{|c|}{ Table 6-11 } \\
\hline Left Sup & $\begin{array}{c}\text { Size of } \\
\text { activation } \\
\text { cluster } \\
\text { (voxels) }\end{array}$ & $\begin{array}{c}\text { Center of } \\
\text { activation (MNI } \\
\mathbf{x}, \mathbf{y}, \mathbf{z}\end{array}$ & p-value & $\begin{array}{c}\text { Percent of } \\
\text { active voxels in } \\
\text { VOI }\end{array}$ \\
\hline Fear & 140 & $-28,-6,62$ & 0.001 & $3.9 \%$ \\
\hline Surprise & 80 & $-28,-6,62$ & 0.001 & $2.2 \%$ \\
\hline Hарру & 233 & $-28,-6,62$ & 0.001 & $6.5 \%$ \\
\hline Control & 30 & $-28,-6,62$ & 0.002 & $0.8 \%$ \\
\hline \multicolumn{5}{|c|}{ Right Sup } \\
\hline Fear & 133 & $38,-6,60$ & $0.000^{*}$ & $3.3 \%$ \\
\hline Surprise & 88 & $36,-8,60$ & $0.000 *$ & $2.2 \%$ \\
\hline Нарру & 111 & $38,-8,58$ & $0.000 *$ & $2.7 \%$ \\
\hline Control & 40 & $38,-6,60$ & $0.000^{*}$ & $0.9 \%$ \\
\hline
\end{tabular}


d. Pars Orbitalis: Both left and right pars orbitalis significantly activated to all conditions compared to baseline, except for happy in the right ( $\mathrm{p}<0.05$, uncorrected). Only fear survived small volume correction in the right hemisphere only ( $p<0.05$, corrected). See Table 6-12.

$\mathrm{P}$-values of 0.000 indicate a significance level of $\mathrm{p}<0.001$. $*$ Symbolizes significance at the corrected level.

\begin{tabular}{|c|c|c|c|c|}
\hline \multicolumn{5}{|l|}{ Table 6-12 } \\
\hline $\begin{array}{l}\text { Left Pars } \\
\text { Orb }\end{array}$ & $\begin{array}{c}\text { Size of } \\
\text { activation } \\
\text { cluster } \\
\text { (voxels) }\end{array}$ & $\begin{array}{c}\text { Center of } \\
\text { activation (MNI } \\
\mathbf{x}, \mathbf{y}, \mathbf{z}\end{array}$ & p-value & $\begin{array}{c}\text { Percent of } \\
\text { active voxels in } \\
\text { VOI }\end{array}$ \\
\hline Fear & 69 & $-34,26,-4$ & $0.000 *$ & $4.1 \%$ \\
\hline Surprise & 29 & $-34,24,-6$ & 0.001 & $1.7 \%$ \\
\hline Happy & 11 & $-20,34,-10$ & 0.000 & $0.6 \%$ \\
\hline Control & 111 & $-42,38,-12$ & 0.002 & $6.6 \%$ \\
\hline \multicolumn{5}{|c|}{$\begin{array}{l}\text { Right Pars } \\
\text { Orb }\end{array}$} \\
\hline Fear & 212 & $38,26,-8$ & 0.001 & $12.4 \%$ \\
\hline Surprise & 16 & $28,32,-12$ & 0.014 & $0.9 \%$ \\
\hline Happy & no sig. & no sig. & no sig. & no sig. \\
\hline Control & 141 & $56,36,-4$ & 0.034 & $8.3 \%$ \\
\hline
\end{tabular}


e. Medial Superior Frontal Gyrus: Both left and right medial superior frontal gyrus significantly activated to all conditions compared to baseline ( $\mathrm{p}<0.05$, uncorrected). No conditions survived the small volume correction. See Table 6-13.

\begin{tabular}{|c|c|c|c|c|}
\hline \multicolumn{5}{|l|}{ Table 6-13 } \\
\hline $\begin{array}{l}\text { Left Med } \\
\text { Sup }\end{array}$ & $\begin{array}{c}\text { Size of } \\
\text { activation } \\
\text { cluster } \\
\text { (voxels) }\end{array}$ & $\begin{array}{c}\text { Center of } \\
\text { activation (MNI } \\
\mathbf{x}, \mathbf{y}, \mathbf{z}\end{array}$ & p-value & $\begin{array}{c}\text { Percent of } \\
\text { active voxels in } \\
\text { VOI }\end{array}$ \\
\hline Fear & 36 & $-6,16,42$ & 0.001 & $1.2 \%$ \\
\hline Surprise & 26 & $-6,16,42$ & 0.004 & $0.9 \%$ \\
\hline Нарру & 35 & $-6,16,42$ & 0.002 & $1.2 \%$ \\
\hline Control & 3 & $-4,16,42$ & 0.03 & $0.1 \%$ \\
\hline \multicolumn{5}{|l|}{$\begin{array}{l}\text { Right Med } \\
\text { Sup }\end{array}$} \\
\hline Fear & 4 & $4,20,44$ & 0.020 & $0.2 \%$ \\
\hline Surprise & 12 & $12,48,46$ & 0.014 & $0.6 \%$ \\
\hline Нарру & 33 & $14,48,46$ & 0.001 & $1.5 \%$ \\
\hline Control & 4 & $10,34,52$ & 0.038 & $0.2 \%$ \\
\hline
\end{tabular}




\subsection{3 fMRI Results Summary}

\section{Table 6-14}

Significant conditions with the largest voxel cluster for each volume of interest. Shaded entries represent left hemisphere volumes.

\begin{tabular}{|c|c|c|c|}
\hline Fear & Surprise & Нарру & Control \\
\hline Left Temporal & $\begin{array}{l}\text { Left Pars } \\
\text { Triangularis }\end{array}$ & Left Fusiform & Left Amygdala \\
\hline $\begin{array}{l}\text { Left Medial } \\
\text { Superior Frontal } \\
\text { Gyrus }\end{array}$ & & Left Parietal & Left Pars Orbitalis \\
\hline Left Insula & & Left Cuneus & $\begin{array}{l}\text { Right Pars } \\
\text { Triangularis }\end{array}$ \\
\hline Left Cingulum & & $\begin{array}{l}\text { Left Middle Frontal } \\
\text { Gyrus }\end{array}$ & Right Parietal \\
\hline Right Amygdala * & & $\begin{array}{l}\text { Left Superior } \\
\text { Frontal Gyrus }\end{array}$ & \\
\hline Right Insula & & Right Fusiform & \\
\hline Right Temporal & & Right Cingulum & \\
\hline Right Cuneus & & $\begin{array}{l}\text { Right Medial } \\
\text { Superior Frontal } \\
\text { Gyrus }\end{array}$ & \\
\hline $\begin{array}{l}\text { Right Middle } \\
\text { Frontal Gyrus }\end{array}$ & & & \\
\hline $\begin{array}{l}\text { Right Superior } \\
\text { Frontal Gyrus }\end{array}$ & & & \\
\hline Right Pars Orbitalis & & & \\
\hline
\end{tabular}

* indicates that contrast fear > motion control was the condition with the largest voxel cluster 
Table 6-15 Hemispheric comparison of each total active voxel percentage for each VOI. Shaded entries represent higher values for between the two VOI hemispheres.

\begin{tabular}{|l|c|c|c|}
\hline VOI & Left & & Right \\
\hline Amygdala & $2.8 \%$ & $8.6 \%$ \\
\hline Fusiform Gyrus & $39.8 \%$ & & $35.9 \%$ \\
\hline Temporal & $11.6 \%$ & & $29.9 \%$ \\
\hline Insula & $12.6 \%$ & $14.2 \%$ \\
\hline Parietal & $63.6 \%$ & & $29.9 \%$ \\
\hline Cingulum & $19.0 \%$ & & $11.9 \%$ \\
\hline Cuneus & $4.9 \%$ & & $2.2 \%$ \\
\hline Par Triangularis & $38.2 \%$ & & $53.7 \%$ \\
\hline Pars Orbitalis & $6.6 \%$ & & $12.4 \%$ \\
\hline $\begin{array}{l}\text { Frontal Middle } \\
\text { Gyrus }\end{array}$ & $3.1 \%$ & & $22.5 \%$ \\
\hline $\begin{array}{l}\text { Medial Superior } \\
\text { Frontal Gyrus }\end{array}$ & $1.2 \%$ & & $1.5 \%$ \\
\hline $\begin{array}{l}\text { Superior Frontal } \\
\text { Gyrus }\end{array}$ & $6.5 \%$ & & $3.3 \%$ \\
\hline
\end{tabular}

Fear

Surprise

$\square$ Happy

$\square$ Control 


\subsection{Discussion}

Unlike our previous two studies illustrating the amygdala's involvement in the processing of threatening (both real and perceived) eye stimuli, no clear pattern of amygdala activation appears with respect to our mouth data. In the right amygdala, there was a slight activation to the happy condition ( 2 voxels) but no significant activation to the remaining three conditions. (Note: in the right amygdala, the contrast of fear > motion control had a considerably larger activation cluster compared to other conditions/contrasts, however this is most likely due to the negative signal change for the control condition compared to that of fear. See Table 6-4). This is most likely due to the fact that a smile is extremely salient in the way that the teeth are suddenly very visible, introducing an attention-grabbing flash of whiteness. On the other hand, the left amygdala responded most significantly to the motion control condition. It is possible that, due to the fearful mouth lacking any sort of salient feature that would specify fear or threat, the implied motion visible in the control condition is novel enough of a stimulus to evoke amygdala activity. Conversely, the same lack of a salient feature can readily explain the indistinct pattern of amygdala activation - a fearful mouth really has no visual properties that pop-out the way that the flash of sclera does for fearful eye whites or the teeth for a happy mouth, thus in isolation a fearful mouth would be less likely to capture the attention of the amygdala.

When in the configuration of the entire face, a fearful mouth yields more of an amygdala response when paired with fearful eyes as opposed to when fearful eyes chimerically appear with a neutral mouth (Morris et al., 2002), underscoring the ancillary role the mouth plays in the expression of fear. Along these lines, Schyns et al. (2007) showed that facial information is integrated starting with the eyes and then moving down the face toward the mouth, irrespective of emotion, and this integration stops when information critical for the behavior (category decision) has been reached. Since the eyes have been shown to be the most important feature of the fearful face, they would be analyzed and trigger a reaction within the amygdala first. The addition of a fearful mouth then, according to Morris et al. (2002), would subsequently heighten this response. Additionally, Gamer and Buchel (2009) presented stimuli where initial fixation was either on the eyes or mouth; for all emotions there was a bias to reflexively shift the gaze towards the eyes, but when fearful mouths were aligned to fixation, amygdala activity was enhanced compared to when the eyes were aligned. Collectively, these studies along with our 
data imply that the fearful mouth alone cannot reliably activate the amygdala but it does play an important role in the context of the entire face.

Nevertheless, the mouth can be a vital diagnostic region for other emotions, such as for happiness where the mean accuracy of recognition nears 100\% (Young et al., 1996), due to the distinctiveness of a smile. Patients with amygdala lesions are still able to recognize happy faces, as amygdala damage predominantly impairs the ability to extract information from the eyes but not the mouth (Adolphs et al., 2005). In the current study, happiness was the most significant of all conditions in eight of the VOIs, but again no clear pattern emerged with respect to the brain regions activated. However, this is not surprising as neuroimaging studies looking at the perception of happy faces reveal no consistencies in activation (Hennenlotter and Schroeder, 2006) instead engaging various regions, many of which are part of the reward system. For the amygdala, reports of signal increases to positive facial expressions (Breiter et al., 1996; Pessoa et al., 2002; Winston et al., 2003) are at odds with studies that do not find activation or have found signal decreases (Morris et al., 1996; Whalen et al., 1998). It is hypothesized that the amygdala plays a more general role in processing facial emotions through modulating levels of vigilance to expressions of both positive and negative affect (Yang et al., 2002; Winston et al., 2003) and not responding specifically to happy faces. So even though a single characteristic can be linked to certain emotions - such as the eyes with fear and the smile with happiness - only fear and disgust seem to have specialized neural representations, while empirical evidence for that of anger, surprise, sadness or happiness is still more limited (Hennenlotter and Schroeder, 2006).

Like the previous experiment, a greater percentage of right hemisphere activation across all VOIs can be seen (Table 6-15) although surprisingly this is not the case in the fusiform gyrus, perhaps indicating that parts of faces do not cause a right hemisphere bias in processing that entire faces do (Sergent and Bindra, 1981; Rhodes, 1985; Luh et al., 1991), at least in the FG. In the temporal VOI, there was a stronger right-side response to all conditions compared to the left, similar to results found by Pelphrey et al. (2005). The most interesting aspect of the temporal results shows a distinct contrast in the pattern of activation in the right hemisphere: the center of activation for both surprise and motion control were the same $(x=46, y=-74, z=-10)$, and were located more posterior and superior to the centers of activation for fear and happiness - which also shared the same center of activation location $(x=44, y=-44, z=-20)$. This could possibly 
be due to the response difference seen in STS neurons to the social observation, intention, and action of others, but no conclusion can be made at this time.

In summary, this experiment revealed that the amygdala does not respond to the fearful mouth when it is presented alone. There is a slight indication that it may respond to stimuli based on their saliency, as the right amygdala activated to the happy condition (smile), leading to the conclusion that the amygdala, specifically the right, responds to stimuli that are particularly pronounced, even if they are not directly associated with fear or threat. 


\subsection{Figures}

Figure 6-1 (23)

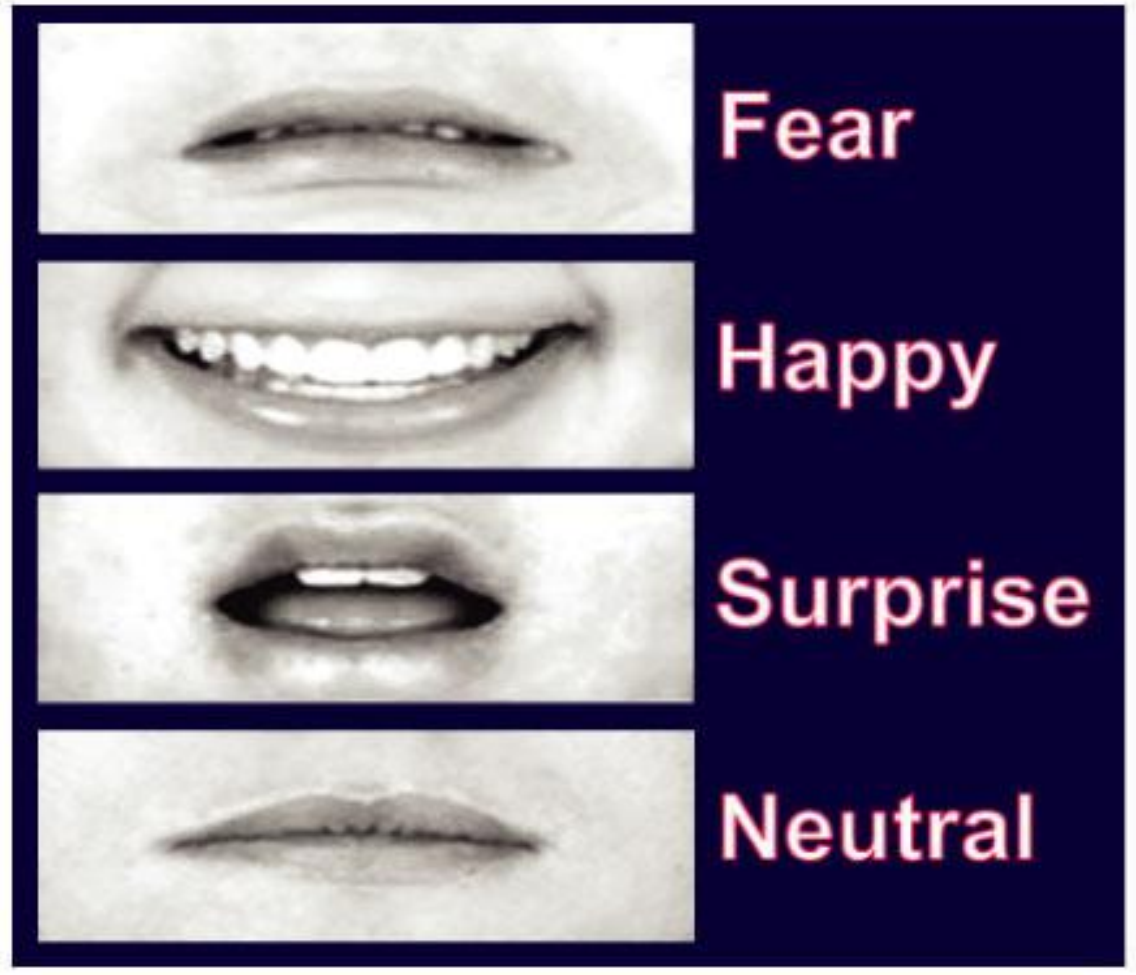

Figure 6-1. Example of stimuli from each condition set. Note how the happy condition elicits the most overall change in form - the mouth widens, the skin dimples around the mouth corners, and the teeth are visible. 
Figure 6-2 (24)

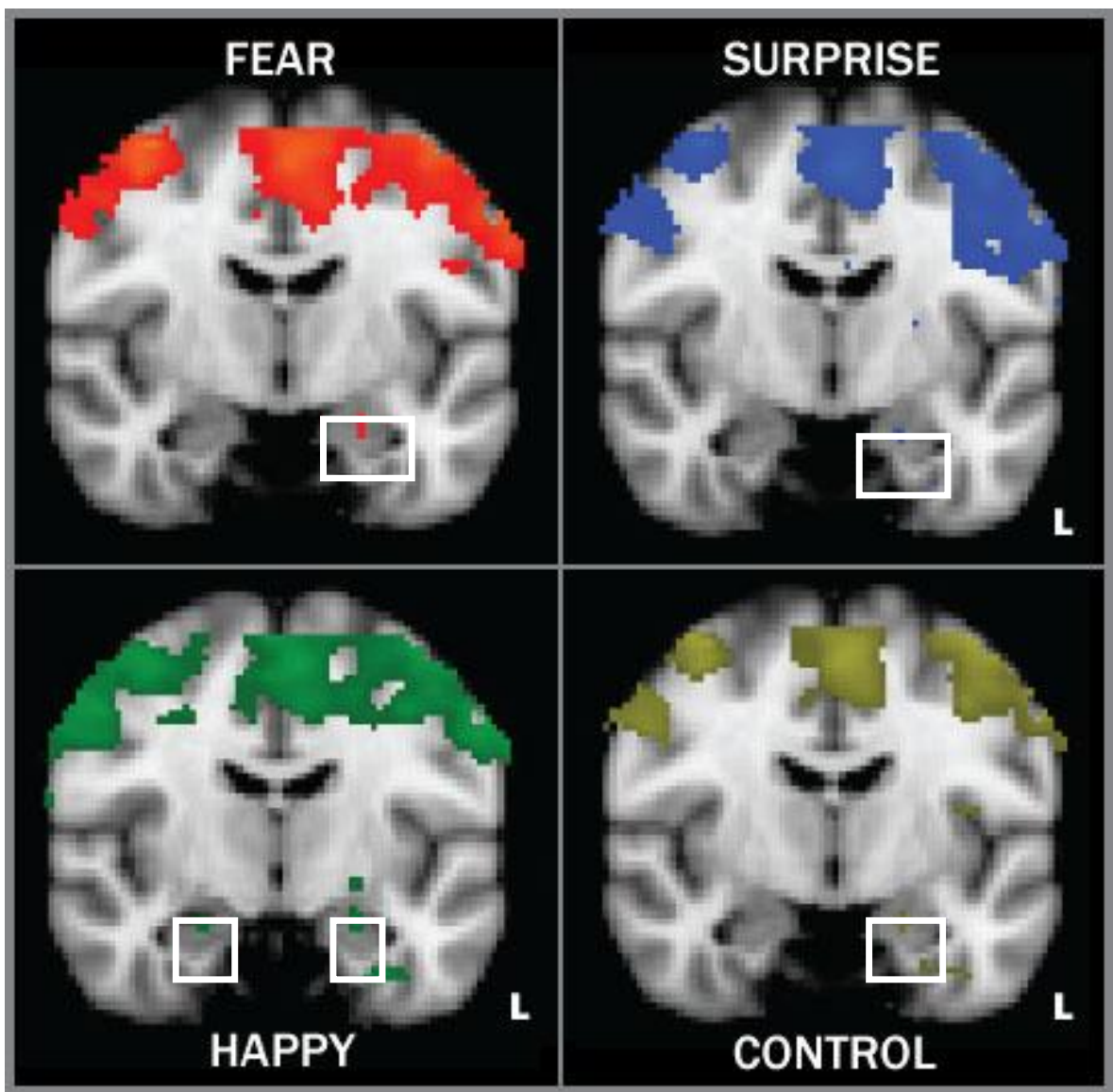

Figure 6-2. BOLD activation in the amygdala (area in box) to each of the emotional conditions averaged across subjects and compared to baseline at $\mathrm{p}<0.05$ uncorrected $(\mathrm{L}=$ left side of the brain). 
Figure 6-3 (25)

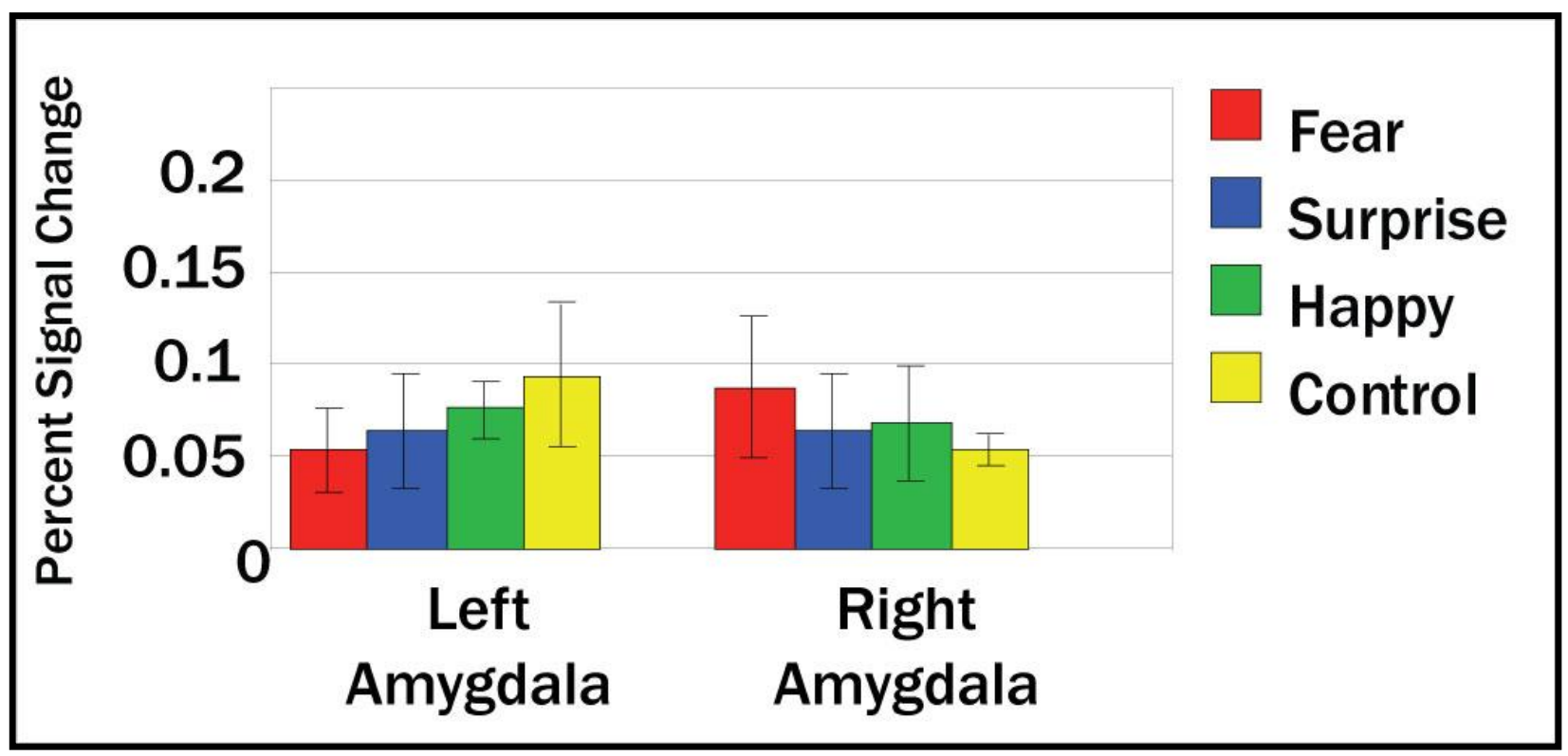

Figure 6-3. Percent signal change from baseline for each condition in the left and right amygdala. There was no significant difference between any of the conditions in either amygdala hemisphere. 
Figure 6-4 (26)

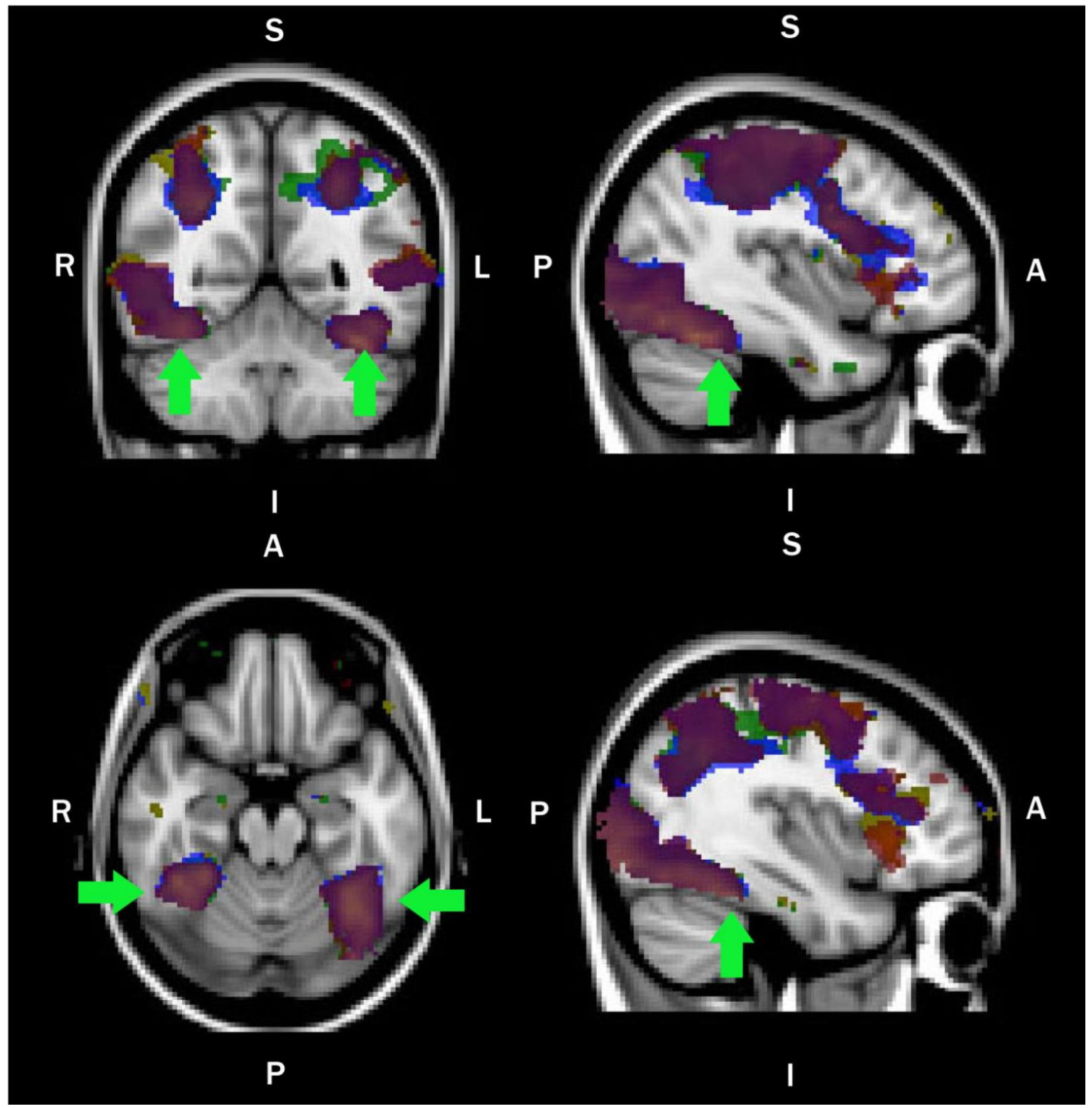

Figure 6-4. Activation to all four conditions at the $p<0.05$ significance level for the fusiform gyrus (green arrows). Fear = red; Surprise = blue; Happy = green; Control = yellow . Areas where color deviates from this scheme indicate overlapping activation for two or more conditions. All four conditions showed similar patterns of activation within the fusiform gyrus. 
Figure 6-5 (27)

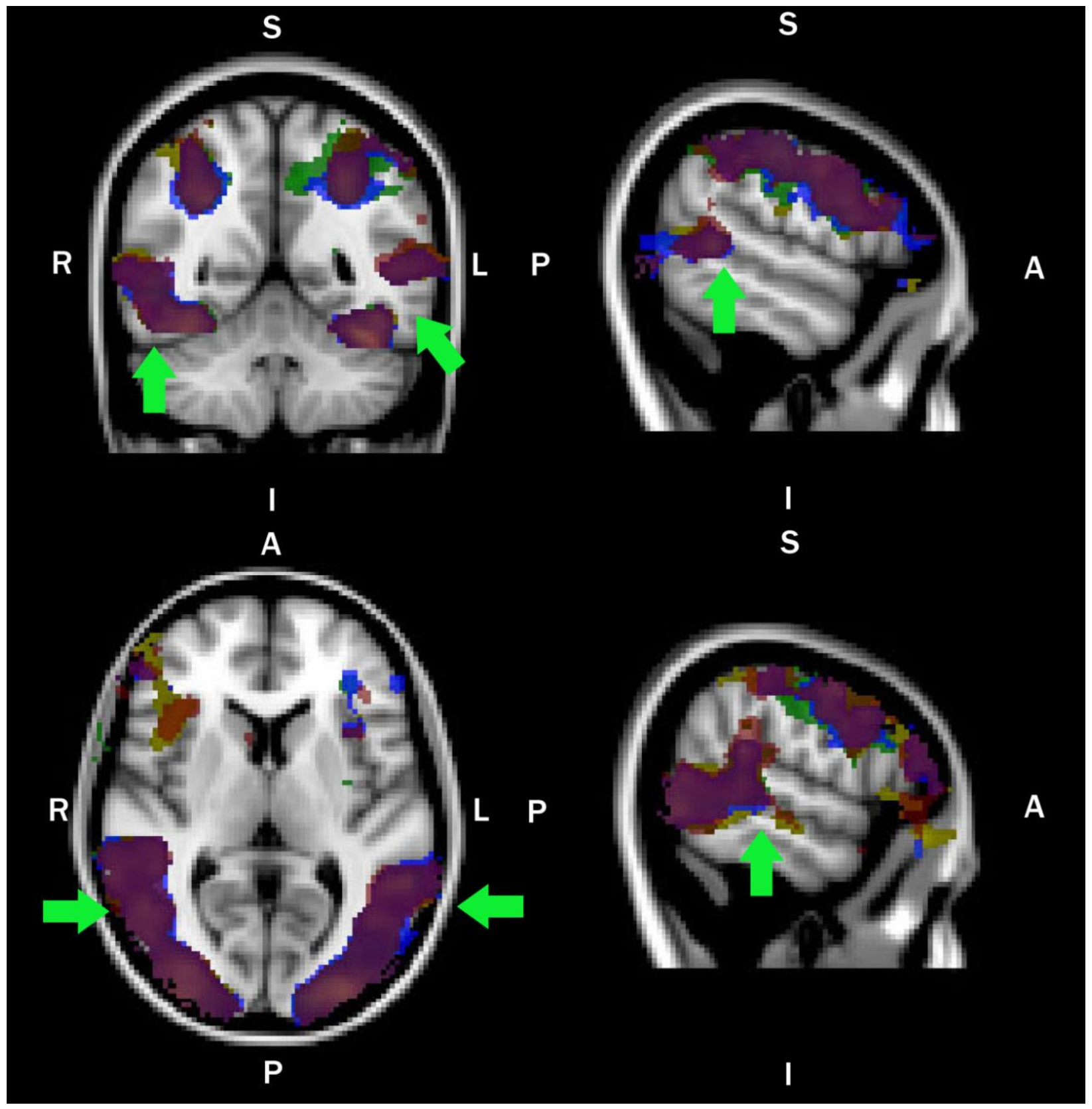

Figure 6-5. Activation to all four conditions at the $p<0.05$ significance level within the temporal VOI. Green arrows highlight areas within this VOI such as the superior temporal sulcus/gyrus and the middle tempral sulcus/gyrus. Fear = red; Surprise = blue; Happy = green; Control = yellow. Areas where color deviates from this scheme indicate overlapping activation for two or more conditions. All four conditions showed similar patterns of activation within the temporal VOI. 
Figure 6-6 (28)

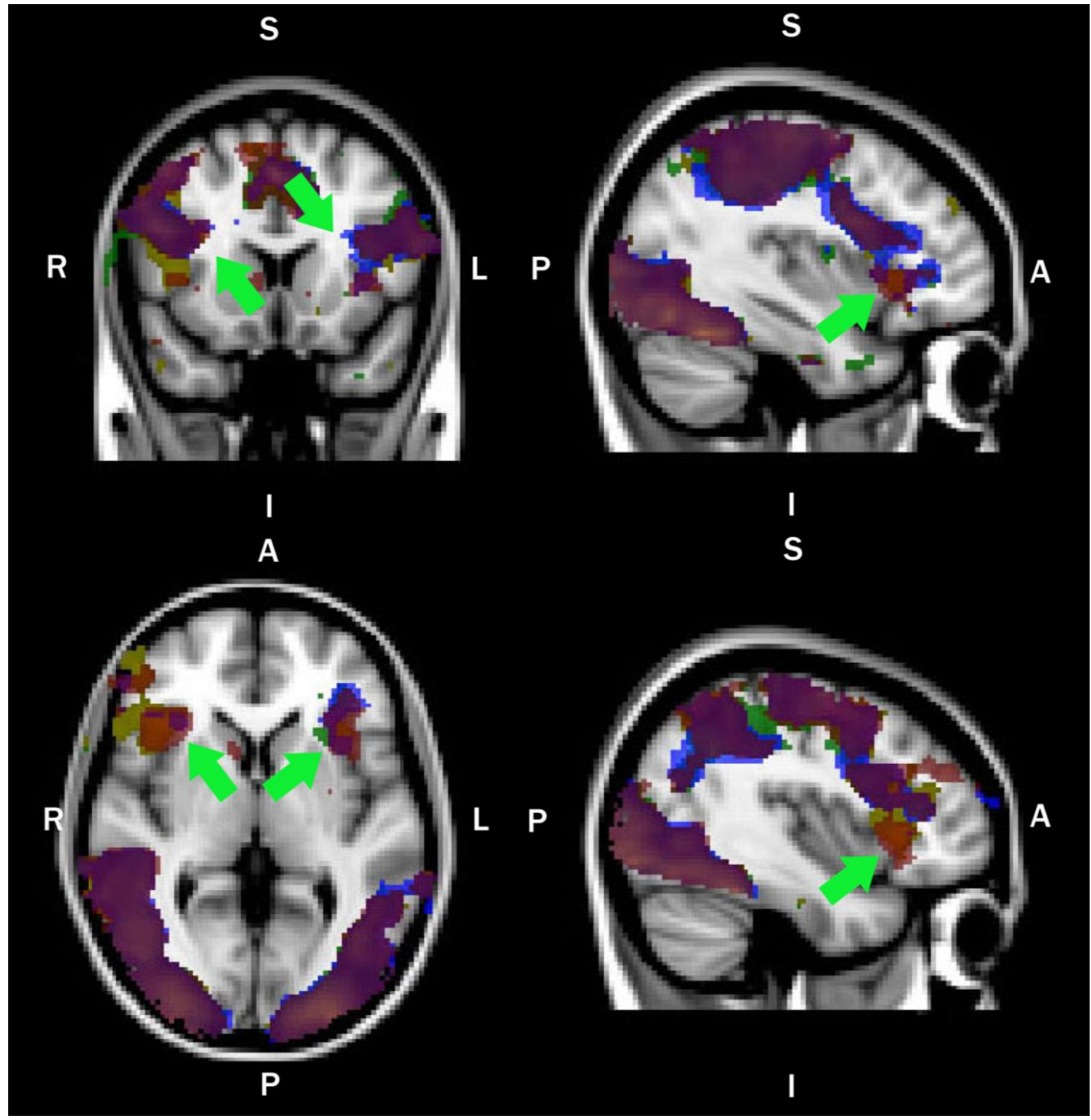

Figure 6-6. Activation to all four conditions at the $p<0.05$ significance level within the insula VOI (arrows). Fear = red; Surprise = blue; Happy = green; Control = yellow. Areas where color deviates from this scheme indicate overlapping activation for two or more conditions. All four conditions showed similar patterns of activation within the insula VOI. 
Figure 6-7 (29)

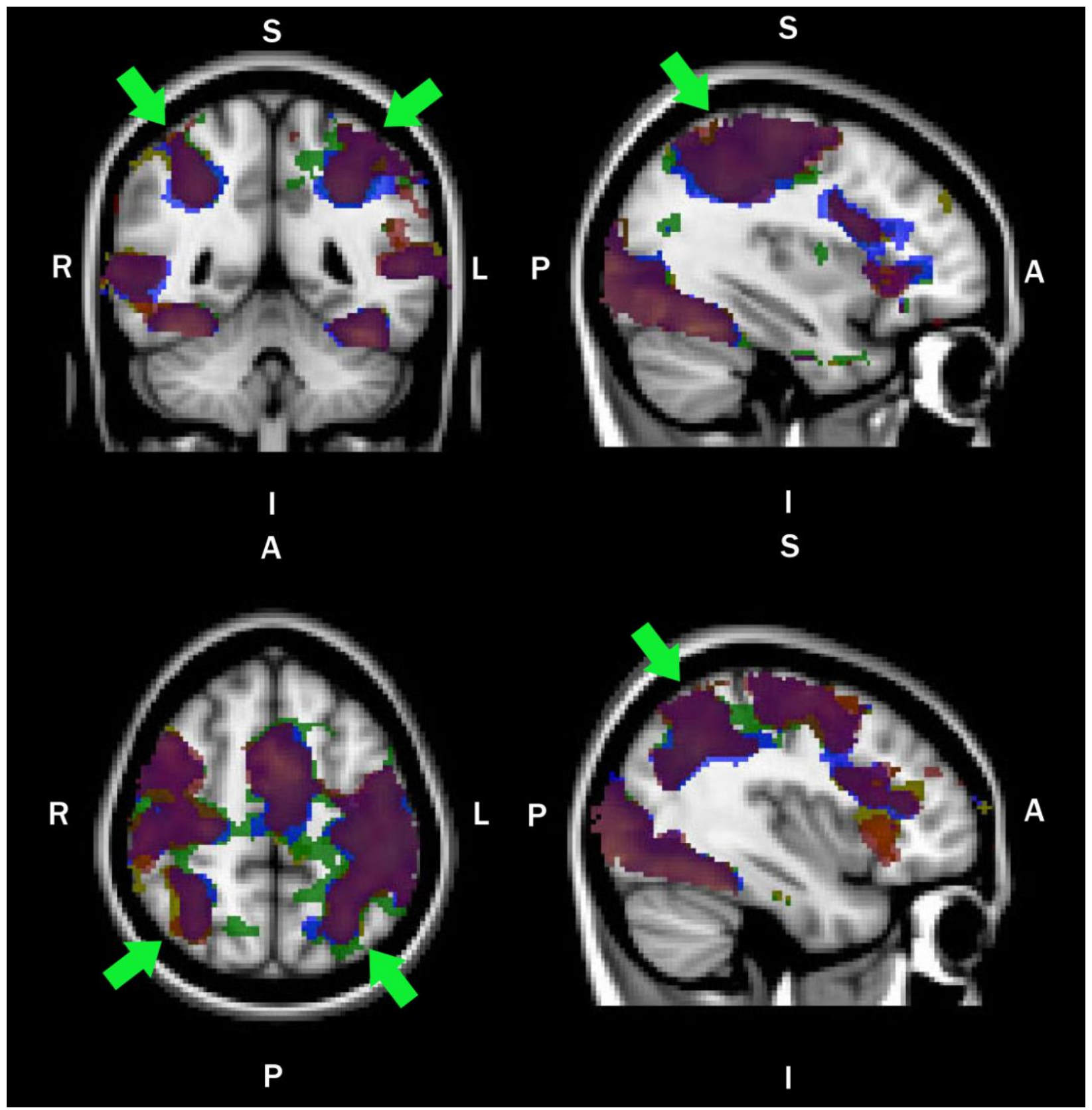

Figure 6-7. Activation to all four conditions at the $\mathrm{p}<0.05$ significance level within the parietal VOI (arrows), highlighting areas such as the IPS. Fear = red; Surprise = blue; Happy $=$ green ; Control $=$ yellow . Areas where color deviates from this scheme indicate overlapping activation for two or more conditions. All four conditions showed similar patterns of activation within the parietal VOI. 


\section{CHAPTER 7: General Discussion and Conclusions}


This chapter summarizes the results across all the experiments and discusses them in their entirety. First, individual regions showing significant findings are examined, including the amygdala (Section 7.1), the fusiform gyrus (Section 7.2.1), and the intraparietal sulcus (Section 7.2.2). Next, patterns of activation across multiple brain regions and how these regions interact is discussed. Finally, the implications of these findings along with general conclusions complete this chapter.

\subsection{Discussion of Amygdala Findings}

\subsubsection{Summary of amygdala results}

The amygdala showed an interesting response pattern for eye stimuli where there was a greater leftward activation for fear (Chapter 4 - Experiment 2) and ambiguous emotions that could potentially signal threat (Chapter 5 - Experiment 3). A slight right-sided laterality effect was visible for the mouth (Chapter 6 - Experiment 4). The following table summarizes the results obtained from the amygdala:

\section{Table 7-1}

\begin{tabular}{|c|c|c|}
\hline Experiment & Amygdala & Results \\
\hline \multirow{2}{*}{$\begin{array}{l}\text { Chpt. 4: eyes } \\
\text { without eyebrows; } \\
\text { compares fear*, } \\
\text { lateral gaze shifts*, } \\
\text { happy*, motion } \\
\text { control* }\end{array}$} & Left & Responded only to fear* \\
\hline & Right & Responded to all four conditions equally \\
\hline \multirow{2}{*}{$\begin{array}{l}\text { Chpt. 5: eyes with } \\
\text { eyebrows; } \\
\text { compares fear*, } \\
\text { surprise*, happy*, } \\
\text { motion control* }\end{array}$} & Left & Responded most significantly to happy* condition \\
\hline & Right & $\begin{array}{l}\text { Responded to happy* condition; not as great as left } \\
\text { activation to happy }\end{array}$ \\
\hline \multirow{2}{*}{$\begin{array}{l}\text { Chpt 6: mouth; } \\
\text { compares fear*, } \\
\text { surprise*, happy*, } \\
\text { motion control* }\end{array}$} & Left & Greater response to motion control condition* \\
\hline & Right & $\begin{array}{l}\text { Responds significantly to happy* condition only ( } 2 \\
\text { voxels) }\end{array}$ \\
\hline
\end{tabular}




\subsubsection{Eyes versus mouth}

Summary of data presented in Experiments 2,3, and 4:

1. The eyes are the main facial feature used by the amygdala to detect threat or fear in another's face

2. The amygdala is unable to identify fear and discern it from other emotions using only the mouth

The results presented here are in line with previous studies that indicate the eyes are more critical for signaling fear than the mouth, both behaviorally and with respect to the amygdala. Smith et al. (2005) illuminated the importance of the eyes for discriminating fear from other emotions when looking at which face regions are optimally used for identifying certain emotions, as did Adolphs et al. (1994) showing that patients with amygdala lesions were deficient at recognizing fear from faces because they were unable to use information displayed by the eyes (Adolphs et al., 1994). The response of the amygdala to fearful faces can be modulated by changes in gaze direction (Adams et al., 2003), as well as by pupil size (Demos et al., 2008), and overall the eye region is more capable of eliciting amygdala activation, even when presented very briefly and without the subject's conscious knowledge (Morris et al., 2002; Whalen et al., 2004). Adolphs (2005) demonstrated how subjects with bilateral amygdala damage were unable to spontaneously fixate on the eye region of faces and instead have a bias for fixating on the mouth (Spezio et al., 2007). Therefore, if amygdala damage causes a lack of fixation on the eyes and these patients cannot identify fear using only the mouth, then it stands to conclude that the mouth does not lend sufficient information for discriminating fear from other emotions, at least from the standpoint of the amygdala.

Our data also indicate the idea that useful information is lacking from the fearful mouth in order to discern fear, as we did not see any clear patterns of activation or a strong bias for fear over our other conditions. It is possible that humans are not effective at identifying fear from the mouth because the eyes are such a salient stimulus in the way that the increase in eye white area "pops-out" at the viewer, contrasting with the darkness of the pupils which are also more visible when the eyes widen, and thus the mouth is disregarded. The mouth may serve more as a secondary indicator confirming that the emotion being expressed is in fact fear, as shown by 
Morris et al. (2002) where emotion recognition increased when subjects viewed fearful eyes paired with a neutral mouth (compared to neutral eyes paired with a neutral mouth), and then increased even more when the fearful mouth was paired with fearful eyes. It is likely that one could learn to use mouth information to correctly identify fear, however in the rare occasions that we encounter a look of fear, it is more likely that the eyes are also visible. Therefore conclusions about whether fear could be recognized solely from the mouth are still preliminary. Further studies, especially using subjects with unilateral or bilateral amygdala damage could shed more light on this topic.

Showing facial features that are not in the context of the whole face enables these face parts to be studied in isolation. However the results can only be interpreted to a certain extent as it is likely that information from the entire face contributes greatly in the accuracy of correctly identifying emotions. With respect to fear, studying the eyes in isolation allows for a better understanding of what elements - such as an increase in eye white area, pupil size, and gaze direction - are able to activate the amygdala and drive the fear response, as well as how to interpret processing deficits present in individuals with amygdala damage. This could lead to better strategies for teaching patients with amygdala lesions to attend to the eyes: when directing them to focus on the eye region, these patients are able to identify fear at a level similar to individuals without amygdala damage (Adolphs et al., 2005). Additionally, encouraging them to use the mouth region to identify fear could also aid in a higher level of accuracy when viewing these emotions. Thus, studying face parts in isolation allows for a better understanding of the mechanisms behind the recognition of particular expressions and allowing for a clearer identification of what brain regions to pinpoint when deficits in processing occur.

\subsubsection{Threat or novelty detection?}

Summary of data presented in Experiments 2, 3, and 4:

1. The amygdala is not only able to act as a detector of fear (Chapter 4), but also as a detector of ambiguity (Chapter 5) as well as stimuli that have salient properties (Chapter 6)

It is well known that the amygdala is central in propagating the physiological fear response seen to dangerous or threatening situations and in fear conditioning paradigms (for a review, see LeDoux, 2007). It was later demonstrated that the amygdala consistently responded 
to fearful faces, therefore the amygdala became labeled as a 'detector of threat' considering the large role it plays in the processing of and response to fearful stimuli. The amygdala also responds to other threat-related expressions, such as anger (Blair et al., 1999; Whalen et al., 2001; Adams et al., 2003), and fearful body positions (Hadjikhani and de Gelder, 2003). These attributes further enforce the idea that the amygdala serves to vigilantly scan the environment for danger and then respond accordingly. However, a growing body of evidence has more recently expanded the duties of the amygdala outside the realm of detecting threat, suggesting that it may play more of a role in detecting salient and novel stimuli and not specifically fear or danger per se.

A study by Herry et al. (2007) illustrates this point using auditory stimuli where both mouse and human amygdalae were more responsive during the presentation of unpredictable versus predictable tones, even though these tones had no biological relevance to either species. In addition to unpredictably, ambiguity also activates the amygdala, as shown in Experiment 3 and in other studies where facial expressions that are not clearly identifiable elicit increased amygdala responses (Whalen et al., 1998; Kim et al., 2003). The amygdala also comes into play when stimuli are salient, no matter if they signify danger or threat (as with fear and anger) or pop-out and easily grab attention, such as sounds that increase in intensity (Bach et al., 2008). Similarly, novel stimuli also captivate the attention of the amygdala, such as in Go-NoGo tasks where NoGo stimuli infrequently appear (Ousdal et al., 2008). The amygdala is thought to first detect the presence of a novel or salient stimulus and then send this information directly to the cortex in order to facilitate attention and perception (Armony et al., 1997; Whalen et al., 1998; Amaral, 2003); the quick response of the amygdala is done prior to awareness and is rather automatic (Whalen et al., 1998).

The results from Experiment 4 attest to a higher level of functionality within the amygdala; if it were just a crude detector of stimuli that are threatening, novel, or salient, then one would expect to see a large increase response to a happy mouth in the amygdala. A happy mouth reveals teeth and a large area of white teeth which would be very salient and attentiongrabbing. There was an increased response in the right amygdala to happiness, however it was localized to only 2 voxels, exhibiting that perhaps the amygdala did find this condition slightly alluring, but through further analysis or cortical feedback did not deem it threatening. Therefore, it can be said that the role of the amygdala is multi-faceted based on the follow arguments: 1) 
novel stimuli do activate the amygdala, but repeated exposure reduces this response in the case where there are no consequences stemming from the stimulus presentation; 2) certain stimuli or ones similar to them that have been negatively encountered before are able to trigger a strong amygdala response; 3 ) the amygdala has a certain, although possibly crude, representation of what is considered a threat due to its ability to selectivity attend to or ignore certain salient stimuli (for example, the configuration of two side by side white discs with dark centrallypositioned circles represent fearful eyes, while the whiteness of the happy mouth is a singular feature and differs greatly in configuration as well as in emotional meaning). In Experiment 3, the response of the amygdala to fearful eyes is greatly diminished compared to the results seen in Experiment 2. Using the idea of novelty and habituation, these results can be explained by the differences in the stimuli that were used. Fear, happy, and motion control eyes were used in both however in Experiment 2 there were eyes depicting a lateral shift in gaze and Experiment 3 used surprise instead. The aim of the Experiment 2 was to compare the amygdala's ability to discriminate between eyes that yielded similar increases in eye white area but differed with respect to configuration and emotional meaning. Experiment 3, however, used two conditions that were identical in eye white area increases and configuration (fear and surprise) and only differed with respect to the position of the eyebrows. Given that it is known that the amygdala will quickly habituate to the repeated exposure of stimuli, it can be argued that its decreased response profile to fear and surprise compared to happy reflects the identical representation of fear and surprise to the amygdala and thus the signal for these two conditions would diminish more quickly. Additionally, the amygdala's inability to disentangle fear from surprise suggests that the eyebrows are not an important feature used to discriminate fear from other similar eye expressions.

\subsubsection{Amygdala laterality}

Summary of data presented in Experiments 2, 3, and 4:

1. Effects of laterality are strongest when identifying fearful eyes - the left amygdala acts as a specific detector of fear while the right amygdala acts as a general detector of change (Chapter 4)

2. Left-side specificity is still present when eye expressions are ambiguous in nature but could potentially signal threat (Chapter 5) 


\subsection{4a General theories of functional laterality in the amygdala}

There are many theories regarding the functional laterality of the brain in general and this also extends into the amygdala, although effects of laterality in the amygdala are not always consistent. However, the most prominent body of research agrees with our data showing a leftsided specificity and a right-sided generality in activation. Markowitsch (1998) proposed that the left amygdala has a higher affinity for the extraction of feature detail, while the right amygdala mediates a gross and quick analysis of affective-related information but is more strongly engaged. Glascher and Adolphs (2003) expounded upon this theory by stating that the left amygdala is involved in a sustained emotional reaction that decodes variations in arousal, while the right is involved in initiating a global emotional reaction triggered by any stimulus that is arousing. This is in agreement with results from Morris et al. (1999) illustrating a rapid and nonselective detection mechanism for the right amygdala by stimuli that could potentially pose a threat to the observer, and the discrimination between different emotional expressions by the left amygdala (Morris et al., 1996; Kim et al., 2003; Whalen et al., 2004). The right amygdala is also susceptible to faster rates of habituation than the left (Wright et al., 2001), activating quickly but then fading out while the left amygdala maintains a sustained level of activation.

The data presented here strongly support these theories of functional amygdala laterality. First, the results outlined in Experiment 2 indicate an overall general level of activation to changes in eye stimuli in the right amygdala: the transition from the neutral eye expression to any of the four conditions (fear, gaze shift, happy, motion control) elicits a physical transformation, which would be salient to the right amygdala. The left amygdala showed a preference for the fear condition only, and its response to all the other conditions was barely discernible, in agreement with the idea that the left amygdala is involved in a more detailed level of feature extraction and can sustain its evaluation. In Experiment 3, even though the left amygdala reacted to all conditions, its response to happiness/anger was far greater. In this instance, instead of being specific for fear, the left amygdala was reacting to a stimulus that was ambiguous in meaning, therefore showing a response pattern like Experiment 2 where the activation to one singular condition was far greater than that of the other conditions. The amygdala's response could infer two different mechanisms for processing the ambiguity in the stimulus: 1) the amygdala defaults toward the more threatening emotion and its sustained 
reaction reflects a heightened level of awareness and/or 2) the condition is truly ambiguous to the amygdala, therefore its activity reflects a need to recruit higher visual areas so that more information can be garnered.

As for the right amygdala, it responded equally to all four conditions without a propensity for one over another (Experiment 2) indicating that it can act as a coarse and non-selective detector of change, regardless of the emotional and behavioral significance behind the change. If it reacts as rapidly and automatically as hypothesized, then it would lack the sufficient detail needed to discriminate between conditions but would serve to heighten the levels arousal and awareness in the brain in general in order to evade potential danger. At the same time, the left amygdala would provide a more accurate determination of whether there actually was a threat or not. In Experiment 3, the pattern of response for the right amygdala differed in that it did show a preference for one condition over the others (happy) but also responded to the other conditions. In this case, if the happy condition was indeed ambiguous in meaning, the right-sided specificity could reflect feedback from other brain regions that are involved in a more detailed processing and are attempting to gather more information about the stimulus.

The absence of a strong lateralized response to the mouth stimuli could simply reflect the lack of importance the mouth region plays in signaling threat. If the amygdala is responsible for directing attention automatically to the eye region of the face, it may not have a propensity for processing information from the mouth and would therefore not prefer one mouth expression over another. There is the slight suggestion of lateralization effects for the left amygdala, as a higher number of voxels responded to the motion control condition compared to the other conditions. This could stem from the puzzling nature of seeing a mouth translate up or down with the viewing area, compared to the implied motion when the other conditions (fear, surprise, happy) change from neutral but remain central in the viewing area. In this sense, the motion control stimuli could appear to be ambiguous, novel, and salient to the left amygdala explaining its preference for this condition. A small lateralization to the happy condition in the right amygdala was present ( 2 voxels in size), which is in line with evidence supporting the right amygdala as a general detector of change or salience, however this response was not large enough to make strong conclusions. 


\subsection{4b Limitations}

It is necessary to point out that effects of laterality in the amygdala literature could reflect the vast differences that exist between studies. Several publications attempt to tackle these issues; the results are not consistent or entirely conclusive. Baas et al. (2004) found a larger number of left amygdala activations in emotional processing, but this was not significantly related to stimulus type (language versus images), task instruction (implicit or explicit) or rates of habituation. They concluded that the left amygdala is involved in local, detailed processing while the right amygdala is involved in more global processing. Zald and Pardo (2002) did find evidence to support theories of amygdala habituation to the repeated presentation of stimuli, similar to that of Wright et al. (2001), theorizing that novel stimuli may actually drive a sustained response in the amygdala. Since the right amygdala is thought to habituate at a faster rate than the left, this would explain the greater reports of left-sided amygdala activation in emotional processing found by Baas et al. (2004) in their review. Future studies could address amygdala habituation within the realm of the studies described here in two ways: 1) present conditions in a block design (the repeated exposure of the same condition continuously within a contiguous block of time) and then compare the activation at the beginning and end of each block for both the left and right amygdala to see if differences in signal change occur, and 2) combine fMRI and electroencephalography (EEG) imaging. This integrates the high spatial resolution of fMRI with the high temporal resolution of EEG so that the response profiles of the left and right amygdala could be directly compared for differences in activation onset time, length of response, and the time at which activity begins to diminish.

Zald (2003) speculated that laterality reflects gender; this has also been reported in other studies showing a right, but not left, amygdala response in men for enhanced memory of emotionally arousing films and a left, but not right, response for women (Cahill et al., 2001), however this effect was not found in the Wager et al. (2003) meta-analysis. Anatomical analyses do suggest that the amygdala as a whole may be larger in men than in women (Goldstein et al., 2001), while imaging studies indicate an overall gender-based lateralization for viewing emotional facial expressions (Schneider et al., 2000; Kilgore and Yurgelun-Todd, 2001; Thomas et al., 2001). For the experiments presented here, we did not look at gender differences with respect to amygdala activation as there was not enough statistical power to discern any. 
Other complications with interpreting lateralized results in the amygdala come from differences in scanning capabilities/sensitivity, data analysis strategies, and the statistical power of subject pools. The amygdala encompasses a small volume of brain space where there are high levels of variability and mislocalization of the magnetic signal due to its close proximity to the sphenoid sinus. This can be overcome by implementing methods that are optimal for imaging the amygdala, in addition to testing these methods out beforehand for reliability. Field strength differences between magnetic resonance (MR) scanners, as well as variations in scanning sequences, also introduce another level of complication when making laterality comparisons across studies; an increase in field strength can allow for greater spatial resolution, but often can be hampered by signal-to-noise issues within the amygdala. In addition, methods for analyzing data and defining the amygdala region introduce more complications in across-study comparisons.

Steps have been taken to minimize dissimilarities across the three fMRI experiments presented here in Experiments 2, 3 and 4. First, all three studies were run on the same 3T scanner using identical scanner protocols that were designed to minimize signal dropout in the amygdala. Next, the same task design and set-up was used, only the stimuli differed. For data analysis, regions of interest were generated in the exact same manner for the amygdala and other brain regions. Despite these precautions, several variations do exist between the studies. The data in Experiment 2 were acquired using a 4-channel headcoil and were analyzed using SPM 2 while data in Experiments 3 and 4 were acquired using an 8-channel headcoil following a MR scanner upgrade and FSL as the analysis package. Nevertheless, these should not cause extreme differences across the data; both SPM2 and FSL are highly-used data analysis packages that virtually perform the same functions based on user input. Additionally, the new headcoil was tested post-upgrade against the pre-upgrade headcoil by a physicist, and scanning parameters were deemed to be the same between the two; spiral in-out sequences were also used for all three fMRI experiments which minimized signal loss from the amygdala. An increase in channel number means an increase in signal-to-noise, therefore the small signal changes that result in the amygdala would more likely be detected, meaning that even though the amygdala data from Experiments 3 and 4 were not as robust as those from Experiment 2, they can be interpreted with the same confidence as the data in Experiment 4 due to the fact that they were acquired using the 8-channel headcoil. 
In conclusion, there is not much concern for comparing laterality effects across these three experiments but instead for comparison across the entire body of amygdala literature, which is just an unfortunate problem that has to be taken into consideration when making broader inferences.

\subsubsection{Other considerations}

It is important to note that while the stimuli used in the Experiments 2, 3 and 4 are categorized as different emotions/expressions (fear, gaze shift, surprise, happy), caution should be taken when interpreting the data based on the labels they have been assigned. The amygdala is essentially detecting certain properties that are associated with certain emotions - such as the widened eyes seen in fear. The experiments presented here sought to study what physical face properties drive the amygdala and so subjects were not instructed to attend to or identify any emotions, thus the amygdala could be responding specifically to the basic physical properties of fearful eyes without regard for what emotional category they represent. Future studies could compare fearful eyes with a cartoon representation of eyes - two adjacent white circles with two darker circles within them - to see if differences exist in amygdala activation between the two. Additionally, running the same experiments presented here but forcing subjects to identify the emotions in the presented stimuli and then comparing them to the results in Chapters 4 and 5 could also shed more light on what is actually driving the amygdala response. 


\subsection{Discussion of Significant Findings in Other Brain Regions}

\subsubsection{Fusiform Gyrus}

\subsection{1a Summary of results}

The patterns of activation in Experiment 2 for the fusiform gyrus (FG) echoed the patterns found in the amygdala: the right FG responded to all four eye conditions equally while the left fusiform responded more significantly to fear compared to the other conditions. In Experiment 3, both the left and right FG activated to all eye conditions equally while in Experiment 4 , activation to the happy mouth condition was higher bilaterally. The following table summarizes results obtained from the fusiform gyrus:

Table 7-2

\begin{tabular}{|c|c|c|}
\hline Experiment & Fusiform & Results \\
\hline \multirow{2}{*}{$\begin{array}{l}\text { Chpt. 4: eyes } \\
\text { without eyebrows; } \\
\text { compares fear*, } \\
\text { lateral gaze } \\
\text { shifts*, happy*, } \\
\text { motion control* }\end{array}$} & Left & $\begin{array}{l}\text { Responded to all conditions; response to fear* was } \\
\text { significantly higher in both hemispheres }\end{array}$ \\
\hline & Right & Responded to all four conditions equally \\
\hline \multirow{2}{*}{$\begin{array}{l}\text { Chpt. 5: eyes with } \\
\text { eyebrows; } \\
\text { compares fear*, } \\
\text { surprise*, happy*, } \\
\text { motion control* }\end{array}$} & Left & \multirow{2}{*}{$\begin{array}{l}\text { Responded to all four conditions equally } \\
\text { On both sides, activation to motion control was } \\
\text { smallest, followed by fear, surprise and happy were } \\
\text { highest }\end{array}$} \\
\hline & Right & \\
\hline \multirow{2}{*}{$\begin{array}{l}\text { Chpt 6: mouth; } \\
\text { compares fear*, } \\
\text { surprise*, happy*, } \\
\text { motion control* }\end{array}$} & Left & \multirow{2}{*}{$\begin{array}{l}\text { Responded to all conditions; response to happy* was } \\
\text { significantly higher in both hemispheres }\end{array}$} \\
\hline & Right & \\
\hline
\end{tabular}




\subsection{1b Discussion of results}

Summary of data presented in Experiments 2,3, and 4:

1. The activity of the left fusiform reflected that of the left amygdala in that it also responded more significantly to fear (Chapter 4) compared to the other three conditions but, unlike the left amygdala, did not show a preference for ambiguous stimuli (Chapter 5)

In the discussion Section of Chapter 4, it was suggested that similar patterns of activation in the left amygdala and left FG, and the right amygdala and right FG, indicate that either area can modulate the activity of the other. The amygdala and FG have a number of anatomical connections between them (Merigan et al., 1991); in macaques there are a higher number of projections from the amygdala to the visual cortex than from the visual cortex to the amygdala (Amaral et al., 2003) and in human subjects, amygdala and FG responses to fearful faces are correlated (Morris et al., 1998). While it was initially thought that the flow of facial information traveled first through higher-level visual areas, such as the FG, and then progressed to the amygdala, more recent data indicates that the amygdala has the ability to modulate FG activity and other regions in extrastriate visual cortex (Vuilleumier et al., 2003). This can explain the parallel activation seen in Experiment 2, however a response similarity does not appear between the amygdala and FG in Experiment 3: while both the left and right amygdala responded preferentially to happy eyes over the other three conditions, neither the left nor right FG significantly preferred one eye condition over another, although there was a slight trend to prefer happiness in both hemispheres.

Despite this lack of comparability, this data provides more evidence that the amygdala can modulate other visual areas and that it can vary this modulation based on its evaluation of the salience of the incoming stimulus. Todorov and Engell (2008) showed that the amygdala responds more to faces that are judged to be negative, and this response increased with higher ratings of negativity. These patterns in the amygdala were positively correlated with activity observed in the FG, however the magnitude of correlation with negative trait judgments was smaller in the FG than the amygdala, indicating that the initial response originates from the amygdala and is then sent to the FG. Moreover, an enhanced response was reported in occipital 
and inferotemporal cortex to emotionally salient but unattended stimuli in patients with hippocampal lesions but not in patients with amygdala lesions (Vuillemier et al., 2004). Since the magnitude of amygdala activation is generally greater for negative than for positive emotional stimuli (Norris et al., 2004; Critchley et al., 2005; Sabatinelli et al., 2005), the significantly heightened activation presented here to fear in the left amygdala and left FG illustrates that the amygdala is tuned to detect fearful eyes - which is a negative emotion - and this can in turn influence activity in the FG. The lack of parallel response between the FG and amygdala in Experiment 3 further illustrates how the amygdala can modify its modulatory effects based on the salience of the stimulus. Ambiguity is not clearly negative, it is only potentially negative. Fear, on the other hand, is absolutely negative and is something that the amygdala is tuned to detect and react to.

In Experiment 4, both the right and left FG preferred the happy mouth over the remaining three conditions, a pattern of activation not seen in the amygdala. The mouth is the primary diagnostic region for the identification of happiness, as a smile is easily and universally recognizable (Ekman, 1970). In general, viewing happiness in another's face does not show any consistencies in the brain regions it activates (Hennenlotter and Schroeder, 2006) and although it does appear that a happy mouth has some effect on the left and right FG here, it is possible that the right FG is affected by the slight activation in the right amygdala. Be that as it may, these results do further support the notion that the amygdala can modulate activity in the FG but does not so for all emotional stimuli, especially those that are efficiently recognized and carry no negative connotation. Furthermore, when the amygdala is not sending modulatory signals to the FG, the FG may not prefer one emotion over another as it has been implicated in face recognition but not necessarily in emotion recognition and identification. In addition, the FG appears to encode faces in a part-based and holistic manner, therefore it can be assumed that the interpretation of these results is not limited by the artificiality of viewing face parts in absence of the entire face (Cabeza and Kato, 2000). 


\subsubsection{Intraparietal Sulcus}

\subsection{2a Summary of results}

The patterns of activation in Experiment 2 in the IPS echoed the patterns found in the amygdala and FG in that there was a trend towards significance in the left IPS to fear. In Experiment 3, both hemispheres activated to all eye conditions similarly while in Experiment 4, the happy mouth condition elicited a higher activation in the left hemisphere. The following table summarizes results obtained from the parietal region:

Table 7-3

\begin{tabular}{|c|c|c|}
\hline Experiment & Parietal & Results \\
\hline \multirow{2}{*}{$\begin{array}{l}\text { Chpt. 4: eyes } \\
\text { without eyebrows; } \\
\text { compares fear*, } \\
\text { lateral gaze } \\
\text { shifts*, happy*, } \\
\text { motion control* }\end{array}$} & Left & $\begin{array}{l}\text { Responded to all conditions; response to fear* trended } \\
\text { to be higher in left hemisphere }\end{array}$ \\
\hline & Right & Responded to all four conditions equally \\
\hline \multirow{2}{*}{$\begin{array}{l}\text { Chpt. 5: eyes with } \\
\text { eyebrows; } \\
\text { compares fear*, } \\
\text { surprise*, happy*, } \\
\text { motion control* }\end{array}$} & Left & \multirow{2}{*}{ Responded to all four conditions equally } \\
\hline & Right & \\
\hline \multirow{2}{*}{$\begin{array}{l}\text { Chpt 6: mouth; } \\
\text { compares fear*, } \\
\text { surprise*, happy*, } \\
\text { motion control* }\end{array}$} & Left & \multirow{2}{*}{$\begin{array}{l}\text { Responded to all conditions; response to happy* was } \\
\text { significantly higher in left hemisphere }\end{array}$} \\
\hline & Right & \\
\hline
\end{tabular}




\subsection{2b Discussion of results}

Summary of data presented in Experiments 2,3, and 4:

1. The activity of the left IPS reflected that of the left amygdala and left fusiform, as it also responded more significantly to fear (Chapter 4) compared to the other three conditions

The intraparietal sulcus (IPS) is located in the parietal cortex and participates in many different attention tasks thought to underlie general visual attention functions, both exogenous and endogenous (Corbetta et al., 1993; Corbetta and Shulman, 2002). Visual attention is controlled by top-down and bottom-up factors; the interaction between these two mechanisms controls where, what and how we pay visually pay attention. Corbetta and Shulman (2002) propose that the detection of novelty and unexpectedness reflects the relationship between topdown and bottom-up factors - otherwise known as cognitive and sensory influences. Studies show that networks recruited for top-down selection can be modulated by the bottom-up specialness of visual objects (Thompson et al., 1997; Bichot and Schall, 1999), especially if they are of relevance or are behaviorally significant (Gottileb et al., 1998). The IPS, in particular, is thought to work as an alerting mechanism and also to provide other areas, such as the temporoparietal junction, with information about the behavioral relevance of a stimulus (Corbetta and Shulman, 2002).

In visual scenes, unattended information usually does not enter awareness and fails to be fully processed, yet emotionally significant stimuli are able to draw in perception and awareness (Öhman, 1986) and the amygdala is thought to be the main player underlying these effects (Vuilleumier, 2005). Some emotional stimuli may be able to affect early perceptual processing (occurring approximately $100-120 \mathrm{~ms}$ after onset) prior to the modulation by voluntary attention (occurring approximately 170 - 300ms onset) (Holmes et al., 2003). Thus, emotional processing in the amygdala can promote the awareness of a stimulus at a particular point in space so that information arising from this point in space can benefit from increased attention by the IPS and then a subsequent enhancement of processing from other visual regions (Whalen and Phelps, 2009). 
In our results from Experiment 2, the IPS responses to fearful eyes trended toward significance in a pattern similar to that seen in the amygdala and fusiform gyrus where activation to fear was higher compared to the other conditions, indicating that the heightened activation within the amygdala could have modulated attentional networks to promote selective attention and awareness. In Experiments 3 and 4, no condition caused a significantly higher amount of activation over the other conditions, however this is not surprising. This could reflect both the nature of the task and type of stimuli used in the Experiments in the sense that subjects were not told to attend to emotion and were not viewing the entire face. Therefore, the increased IPS response to fearful eyes in Experiment 2 could reflect the importance behind recognizing fear in the face of another while Experiments 3 and 4 indicate that the allocation of attentional resources can be modulated by the activity in the amygdala: ambiguity (Experiment 3 ) and information from the mouth may not require as much attention for processing. In addition, all stimuli, as well as the shape task, appeared in the center of the screen so the shifting of vision was not required. Because of this, a lack of significant IPS results for one condition over another in Experiments 3 and 4 could simply reflect the fact that their presentation was in an already-attended location so no additional attention was required to process them, whereas in Experiment 2 the fearful eyes were highly salient and biologically relevant and therefore garnered more amygdala and attention network resources by default.

The centers of activation for the four conditions were spread across both the anterior and posterior IPS for all three Experiments. Imaging evidence supports the existence of two attentional processes in the parietal cortex based on selective attention of motion or feature cues (Shulman et al., 2002), however the present Experiments were not specifically designed to investigate attentional mechanisms and therefore it would be difficult to make any assumptions about the nature of response locations based on condition.

\subsubsection{Activation for brain regions that did not differentiate as a function of stimulus condition (secondary data VOIs plus the STS and insula)}

Data was reported in a number of other brain regions that did not show significant results with respect to Experiments 2, 3, and 4, such as the STS, the insula, and a number of areas within the frontal cortex, particularly the orbitofrontal cortex. All of these regions appeared as VOIs in the data analyses because they have been previously shown to be involved in different aspects 
emotional, social, and face processing however they did not show patterns of activation that reflected what was seen in the amygdala, the FG, and the IPS. The lack of significant results presented here indicate that these areas may not be directly influenced by the passive viewing of face parts, especially when the task involved is secondary to the aim of the experiment. Because attending to emotion can alter neural processing, particularly for fear, a more active task where subjects had to identify the eye and mouth stimuli based on emotion may have biased the responses in these areas and significant results could have developed. Presently, the absence of a preference for one emotion over another in these regions implies that they are more generally involved in social, emotional, and face processing but are not necessarily driven by particular features - such as fearful eyes - like the amygdala. 


\subsection{General Summary}

The following three figures summarize the results for the amygdala, the fusiform gyrus, and the intraparietal sulcus in Experiments 2, 3, and 4 for the visualization of similar activation patterns across these three regions. The results are shown as the percent signal change from baseline for each VOI per condition.

Figure 7-1 (30). Experiment 2 Summary of results for amygdala, fusiform gyrus, and intraparietal sulcus for Experiment 2 (eyes without eyebrows).

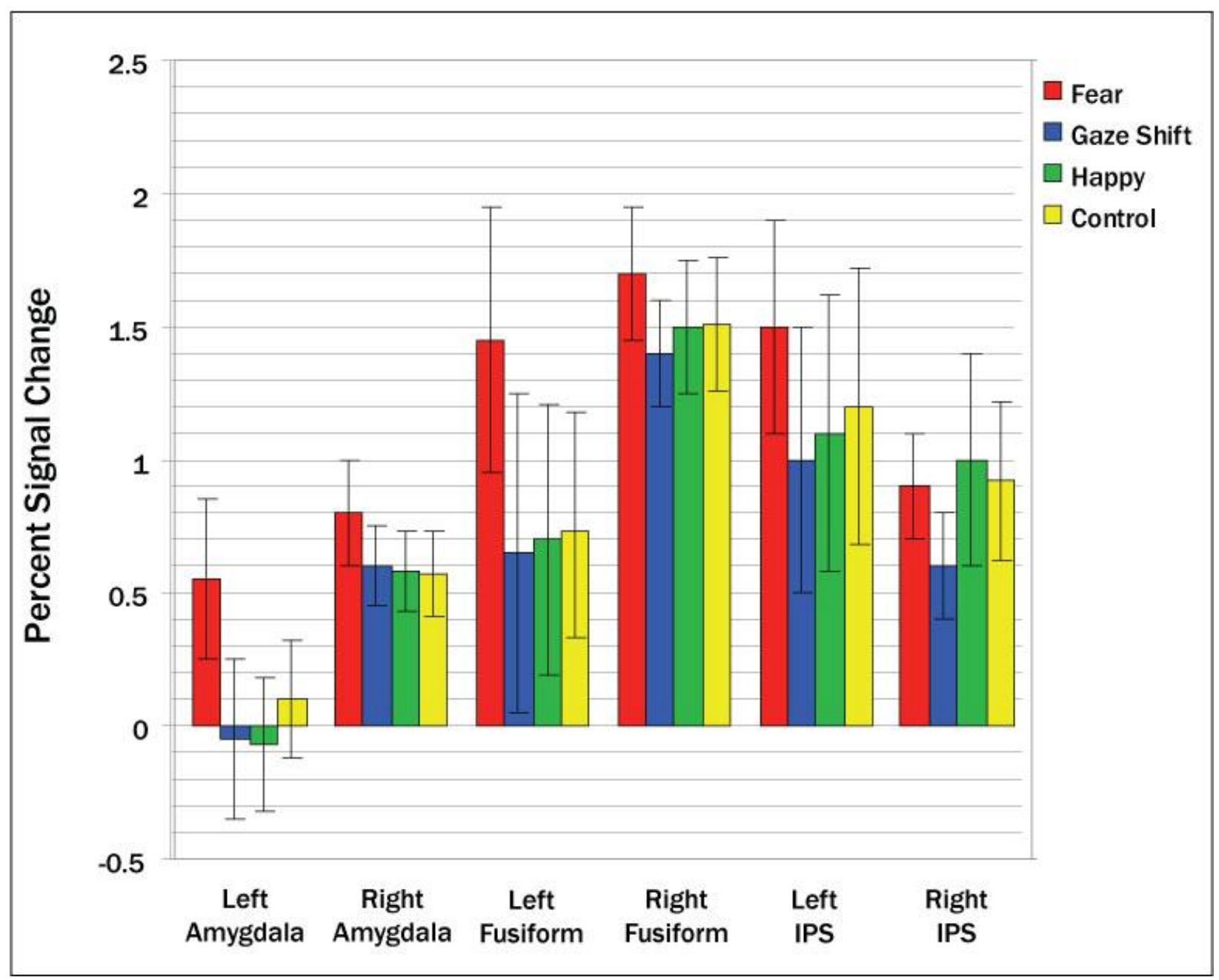


Figure 7-2 (31). Experiment 3 Summary of results for amygdala, fusiform gyrus, and intraparietal sulcus for Experiment 3 (eyes with eyebrows).

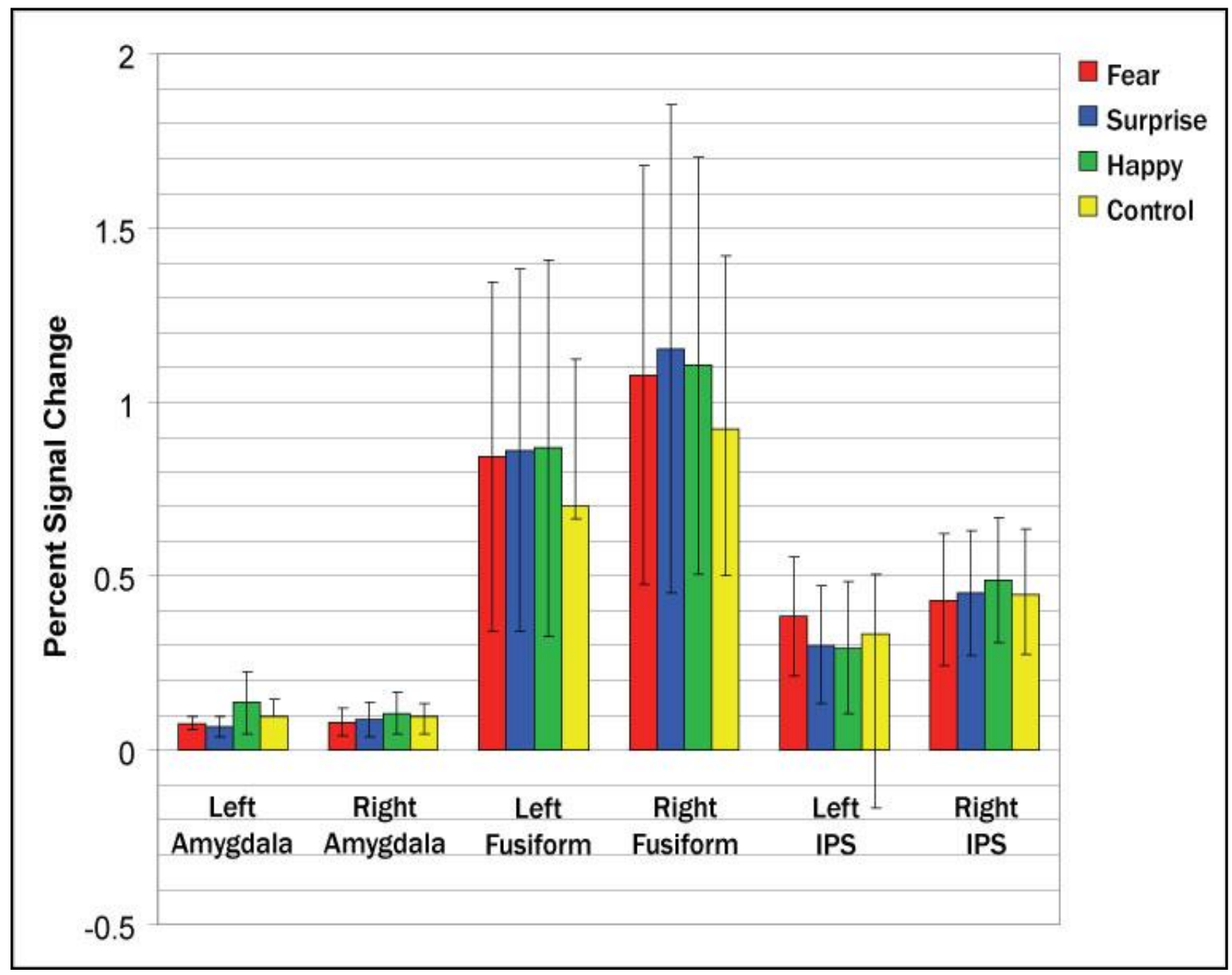


Figure 7-3 (32). Experiment 4 Summary of results for amygdala, fusiform gyrus, and intraparietal sulcus for Experiment 4 (mouths).

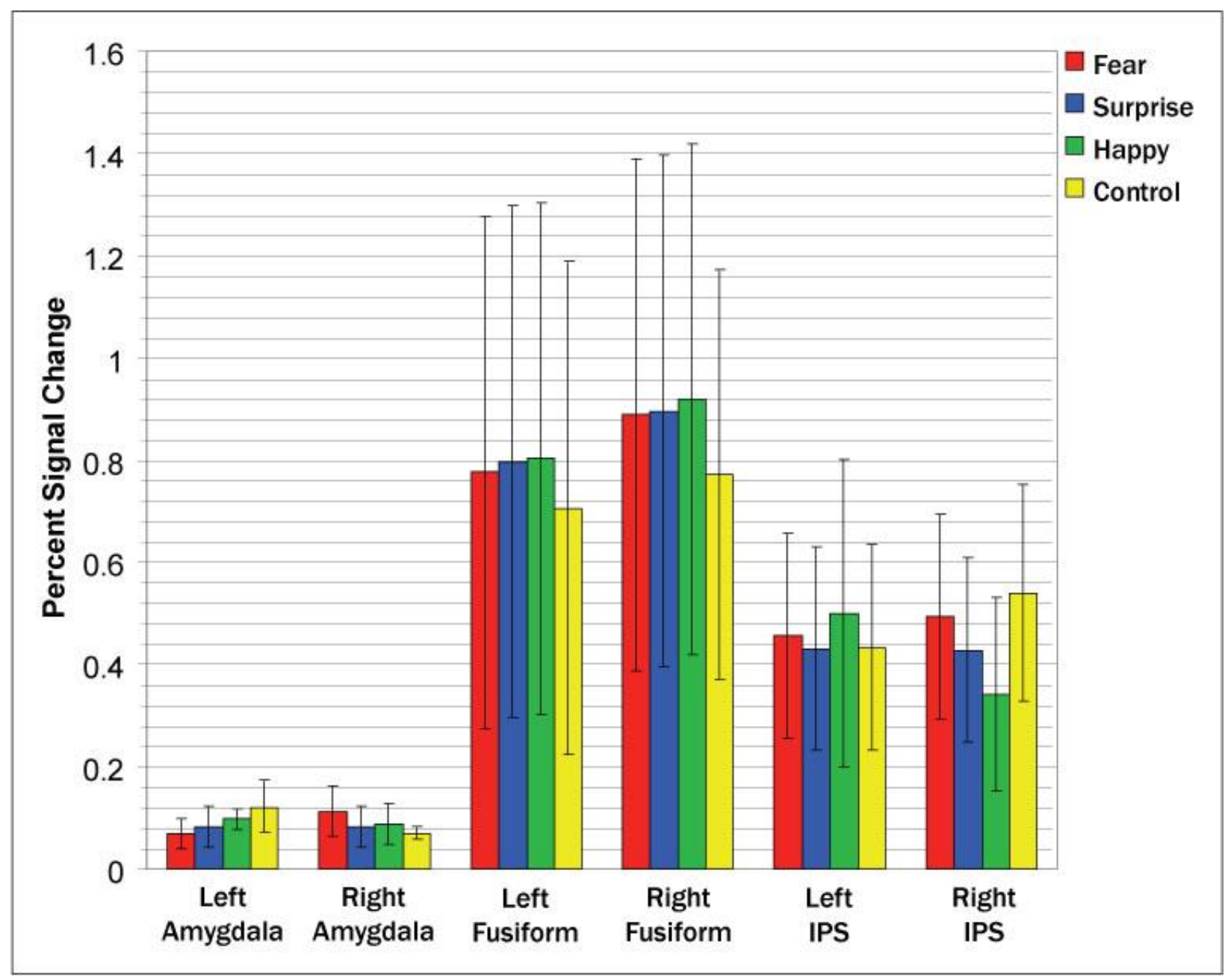

Figure 7-1 illustrates how the left FG and left IPS share the pattern of activation seen in the left amygdala where the response to fear is greater than to the other conditions. Similarly, the right hemisphere also showed a preference for fear over the other conditions however the right amygdala activated to all conditions while the left amygdala responded only to fear. In Figures 72 and 7-3, no clear pattern of activation emerged across the three regions of interest like that visible in Figure 7-1.

In order to explain the results above, it is necessary to review how the amygdala is involved in visual processing. The amygdala is able to receive crude visual inputs via two neural pathways (LeDoux, 1998): a direct pathway (extrageniculostriate) that travels via the superior colliculus, the thalamus, and the pulvinar (de Gelder et al., 1999; Morris et al., 1999, 2001) and 
an indirect pathway (geniculostriate) that travels through the thalamus to the amygdala after it has been processed in the visual cortices (LeDoux, 1998; Adolphs, 2002). One of the key regions identified by Adolphs (2002) that could send information to the amygdala via the indirect pathway is the fusiform gyrus (FG); the FG has shown an increase in activation with increases in the amygdala response to fearful faces (Morris et al., 1998a; Keightley et al., 2003). A subcortical pathway has also been proposed for the processing of faces, involving the superior colliculus, pulvinar, and amygdala (Johnson, 2005). This subcortical pathway is thought to modulate cortical face processing in regions such as the FG. ERP and MEG studies demonstrate that the subcortical pathway is rapid, with latencies less than $100 \mathrm{~ms}$, and operates using lowspatial frequency (LSF) information (Johnson, 2005). Additionally, emotional events are able to enhance processing through prioritizing attention (Fox et al., 2000; Anderson and Phelps, 2001; Vuilleumier and Schwartz, 2001), which is especially true for fear-related and other negative visual stimuli that activate both the amygdala and visual cortical regions associated with emotion. (Lang et al., 1998; Morris et al., 1998a). Vuilleumier et al. (2004) demonstrated that the enhanced responses in other visual cortices to fearful stimuli are dependent upon the amygdala, and relies on the amygdala's evaluation of the stimuli and not any specific features that are extracted in the feedforward stream from the visual cortex.

In Experiment 2, the specificity of the left amygdala for fear indicates that it is able to process eye information on a detailed level. Because of the similarities in activation between the left amygdala and left FG, these two regions may work in tandem to process eye information on a more detailed level as the FG receives sufficient spatial detail and fine-grained inputs from the lateral geniculate nucleus (Merigan et al., 1991). Either of these regions could also alert the IPS in order to heighten attentional resources also as a means to gather detailed information. However, it is unlikely that the amygdala is able to discriminate fear from conditions with similar increases in EWA (such as a shift in gaze, or surprised eyes in Experiment 3) if it relies on LSF information, therefore it is hypothesized that in this case the left FG is able to aid in the amygdala's specific response to fear, suggesting that cortical processing is influencing the amygdala response. Data for the right hemisphere indicates an opposite direction of influence in that FG activity is modulated by the amygdala. The right amygdala has been proposed to act in an automatic and rapid manner (Glascher and Adolphs, 2003) that propagates a general level of arousal in response to stimuli; in Experiment 2 the amygdala appears to detect coarse changes to 
the eye as it activates to all conditions. The heightened activation to fear in the right amygdala paired with the heightened activation to fear in the right FG suggests a subcortical pathway is in operation and that information is flowing first through the amygdala and then progresses on to the FG. Based on the results of this experiment, it appears that the left and right amygdala have evolved to perform different mechanisms for processing fear from the face of others as a subcortical pathway would allow for the rapid detection of potential threat and a cortical pathway would enable an accurate determination of whether the potential threat is real or not.

When not processing stimuli signaling potential threat or danger, these patterns of activation and modulation appear to be different. The magnitude of amygdala activation is generally greater for negative than for positive emotions (Critchley et al., 2005) and can also process different levels of emotional intensity in addition to emotional valence. In Experiment 3 , the amygdala showed an increased response to the happy condition which is thought to represent ambiguity. However, the FG and IPS do not show the same activation patterns as the amygdala, indicating that the amygdala did not modulate these regions based on its own evaluation of the stimuli (note: fMRI data do not easily give information about the time courses of activation, it may well be that there are large differences in timing here that cannot be seen). Comparing these results to that seen in Experiment 2, it can be said that effects of laterality may be useful in potentially threatening situations but are not necessary when interpreting other aspects of emotional expressions, such as ambiguity, or when assessing emotions that are less negative than fear, such as anger. Therefore, the amygdala may only modulate areas of higher visual processing and attention networks - or conversely be modulated by them - in situations that are potentially dangerous and require fast behavioral responses. The interaction and modulation patterns that occur between the amygdala, the FG, and the IPS could be further investigated using EEG alone or in combination with fMRI to elucidate the timing profiles between these regions in order to understand what areas activate in what order. Furthermore, using stimuli made of low and high spatial frequency information, as tested previously by Vuilleumier et al. (2003) using fMRI, in combination with EEG would lend further understanding to how the presence or lack of an amygdala response effects the response timing of the FG and IPS.

In conclusion, the amygdala is not only involved in the detection of fear in the face of another, but also detects facial stimuli that are ambiguous or salient however, some level of prior experience or knowledge can temper the amygdala's reaction. While fear elicits the strongest 
response in the amygdala and is processed in an automatic and lateralized manner adapted for the avoidance of threatening situations, other stimuli that the amygdala evaluates do not always generate strong, automatic, or lateralized responses. This data lends more evidence to support the theory that the amygdala is not a slave to cortical activity, but instead is able to work in tandem and influence other areas of visual processing. 


\subsection{Implications and Future Directions}

As discussed in Chapter 1, amygdala dysfunction is thought to underlie a number of social behavior deficits seen in autism, such as making eye contact with others. People with autism spend more time viewing the mouth and less at the eyes when looking at faces (Pelphrey et al., 2002; Klin et al., 2002). Looking at the mouth may be a strategy that autistic individuals use to extract social meaning as the eyes are less informative to them. Neumann et al. (2006) demonstrated that it is unlikely that their attraction to the mouth is due to its salient features, but may in fact reflect an impaired top-down modulation of attention and not bottom-up attention processing. Johnson (2005) hypothesized that the subcortical face processing pathway is abnormal in autistic individuals and may be less tuned to LSF information and more towards high-spatial frequencies, therefore biasing processing towards that of featural and not configural processing. An abnormality in this system could be responsible for the diminished attention to faces early in life for autistic children (Maestro et al., 2002), and a failure to orient to salient social stimuli such as faces.

The data presented here show differential amygdala responses to the eyes based on the type of information extracted from them (threat, ambiguity) and a lack of amygdala response to the fearful mouth. These differences could be used to better understand why individuals with autism cannot use information from the eyes to detect fear but are able to respond to other emotions. While the findings presented here do not directly relate to autism, they do illustrate the distinct response profiles that amygdala exhibits to different emotional face parts and how effects of laterality do not always extend to every type of evaluation that the amygdala makes. Attempting to replicate these experiments in individuals with autism may lend to the greater understanding of their processing deficits, whether one amygdala hemisphere is able to compensate for the lack of activation in the other, and how the absence of an amygdala response to certain stimuli (eyes, mouth) affects other significant visual areas, such as the FG and IPS. Furthermore, would the lack of amygdala activation cause an increased processing load for other regions that were not found to be significant here, such as the STS and insula? Would individuals with autism activate differently to mouth stimuli compared to motion control subjects due to their abnormal face processing patterns? Understanding the normal limits of amygdala function could greatly aid in understanding the anomalies seen in autism. 
In addition to studying how the amygdala processes emotional face parts in autistics, further elucidating the relationship between the amygdala, the FG, and the IPS in control subjects would shed light on the direction of the flow of information between these regions. Timing profiles in face and emotion processing is still in its infancy with respect to the amygdala, future studies on this would shed light on the nature of feedback / feedforward information and its involvement in subcortical and cortical pathways for face and emotion processing. 


\section{Appendix}




\section{A.1 Functional Magnetic Resonance Imaging}

Functional magnetic resonance imaging (fMRI) is a non-invasive brain imaging technique developed in the early 1990s (Ogawa, 1990). It measures changes in blood flow and oxygen metabolism to infer brain activity, usually in response to a specific task or stimulus. This type of imaging makes use of changes in local magnetic susceptibility between oxyhemoglobin and deoxyhemoglobin that leads to a distortion in the magnetic field. The advantage of fMRI over other brain imaging modalities is that it does not involved the use of radiation, such as with positron emission tomography (PET), and it has excellent spatial and is non-invasive.

In MRI, the hydrogen nuclei of water molecules in the body are the source of the signal that gives an image. When neurons within the brain are active there is an increase in blood flow to this region due to a demand for oxygen and glucose, as firing neurons need energy to be brought in quickly. Oxygenated blood, called hemoglobin, is diamagnetic while deoxygenated blood, or deoxyhemoglobin, is paramagnetic. The differences in magnetic properties between the two causes differences in the MR signal of blood due to the effect that each has on the spins of local water protons, this is referred to as blood oxygenation level dependent (BOLD) imaging, When neural activity increases, there is a brief decrease in blood oxygenation that is followed by a period of increased blood flow that overcompensates for the local neuronal oxygen demand, which typically peaks around 4-6 seconds following the onset of local neural activation (Ogawa, 1990). The differences in signal are detected by the MR imager and are constructed into an image using Fourier analysis; raw data consists of intensity values over time at each voxelwhich represents the location of the signal in the relevant tissue in the $\mathrm{x}, \mathrm{y}$, and $\mathrm{z}$ directions (Bracewell, 1986). These intensity values are then compared with values that are obtained during a baseline condition are then compared in order to determine the difference in signal between this baseline and a specific condition, thus enabling an understanding of brain organization and status.

When a person is put into an MR scanner, protons from the hydrogen atoms in water will become oriented with the magnetic field of the scanner. Radio waves are then applied (called the radio frequency, or RF pulse) at an appropriate frequency to change the spins of the protons as they absorb energy. When the RF pulse is turned off, the protons return to the original orientations and energy is emitted as radio waves. T1-weighted images measure how quickly the protons realign with the scanner magnet - so that fluid appears very dark while fat is very bright 
- and T2-weighted images measure how quickly energy is given off by the protons as they return to equilibrium, but this time fat appears dark while fluid appears bright. Gradients are used to encode space, thus magnetic field gradients create different frequencies that affect protons in different parts of space. The information about frequencies in an image are contained in what is called k-space. Different types of acquisition sequences sample k-space in distinct ways: echoplanar imaging collects this data in a zigzag direction while spiral imaging does it in a spiral manner. Repetition time, or TR, is the time between subsequent RF pulses; variations in this help control characteristics of image contrast. Gradient echo sequences, like the ones detailed in Chapters 4,5 , and 6 , have a flip angle below $90^{\circ}$ which allows for a faster proton recovery time and thus a shorter TR. When using MR as a tool to probe certain questions about the brain, blood deoxyhemoglobin is used as an endogenous contrast agent to determine areas of increased or decreased activity in response to a certain stimulus, as outlined in the previous paragraph above.

When designing an fMRI experiment, temporal and spatial characteristics of the hemodynamic response must be taken into consideration, as well as the type of task being employed and the psychological question being asked. Therefore, fMRI experiments can be sorted into two general categories: the block design and the event-related design. In block designs, the experimental task is performed for approximately 20-60 seconds of continuous time, or a block of time (Friston et al., 1999). This task design is optimal for detecting small changes in neural activity; however its major weakness is that all stimuli or tasks remain the same within each block, and therefore many classical psychological paradigms cannot use the block design. Event-related designs allow for multiple individual stimulus presentations, as opposed to identical stimuli over a period of time, and the hemodynamic response for each individual stimulus can be measured (Buckner et al., 1996). This design type is particularly useful in experiments where novel stimuli are being presented and gives greater temporal control over the hemodynamic response, whereas the block design assumes that the BOLD response remains constant across the period of interest. However, event-related experiments can be more challenging to set up and analyze. The key to both types of task designs is to suitable "control" condition that serves as a baseline from which to compare stimulus events against.

After imaging data is collected but before it can be analyzed, it undergoes preprocessing - called so because it is done before data analysis. This step allows for the removal of data variability so it does not have to be modeled. Head motion can occur over the course of a 
scanning session, even when every effort has been made to minimize this from occurring. Motion that is not too great can be compensated for in most fMRI data analysis packages; this helps to minimize the appearance of false activation (Woods et al., 1992; Friston et al., 1995, 1996; Woods et al., 1998). Other preprocessing steps include: 1) slice timing correction corrects for differences in acquisition time within a TR; 2) coregistration - allows for activation to be displayed on anatomical images and aids in normalization; 3) normalization - provides coordinate space for result reporting, and enables data to be averaged across all subjects; 4) spatial smoothing - increases signal-to-noise ratio and may improve comparisons across subjects.

To begin data analysis, it must first be determined whether the experimental manipulation caused a measurable MR signal change and if so, where and when did this occur? The most common approach is to use a general linear model, where the fMRI data is compared to a reference temporal function in order to ascertain where high correlations exist between this reference function and the actual data. The reference function comes from the experimental design, and takes into consideration the shape of the hemodynamic response at the time a particular event occurred within the fMRI data. If looking at data collected from multiple subjects, each subject's brain must be transformed into a common space, such as Talairach (Talairach and Tournoux, 1998).

fMRI has been used quite extensively since 1991 across a number of applications, including sensation, perception, attention, cognition, language, and emotion in both normal and patient groups. The methods presented here generally pertain to procedures used in the experiments in Chapters 4, 5, and 6 and do not necessarily represent all types of analysis techniques, yet serve more as an informational accoutrement. 


\section{A.2 PickAtlas Volumes of Interest}

The following images detail the portions of the brain included in each VOI generated by the Wake Forest University PickAtlas tool.

Figure A-1(33)
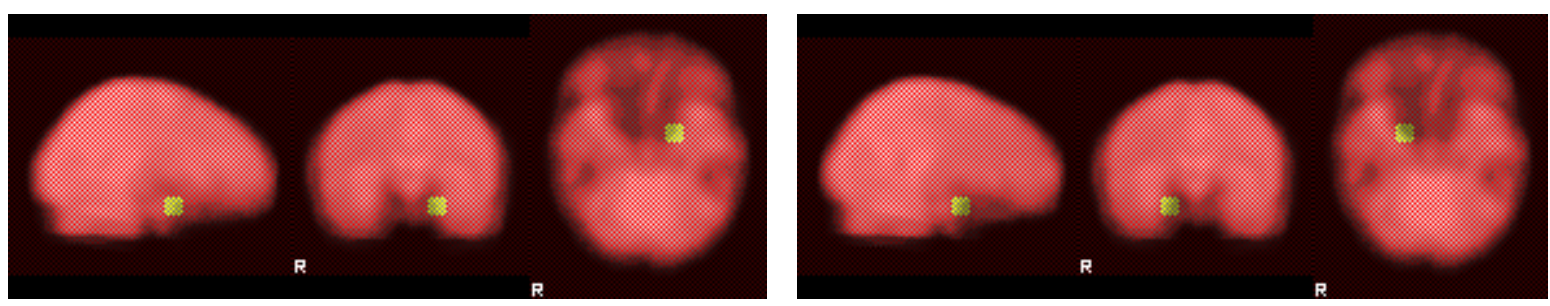

Figure A-1 Left and right amygdala VOIs

Figure A-2(34)
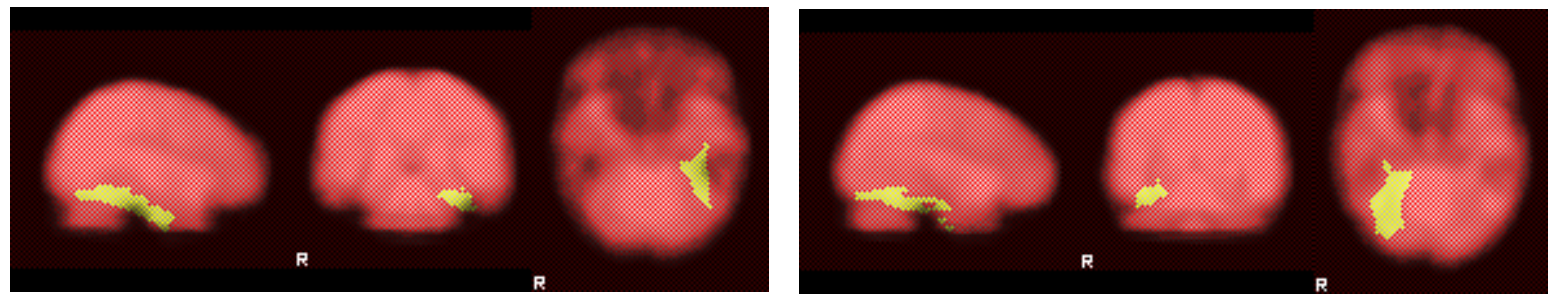

Figure A-2 Left and right fusiform VOIs

Figure A-3(35)
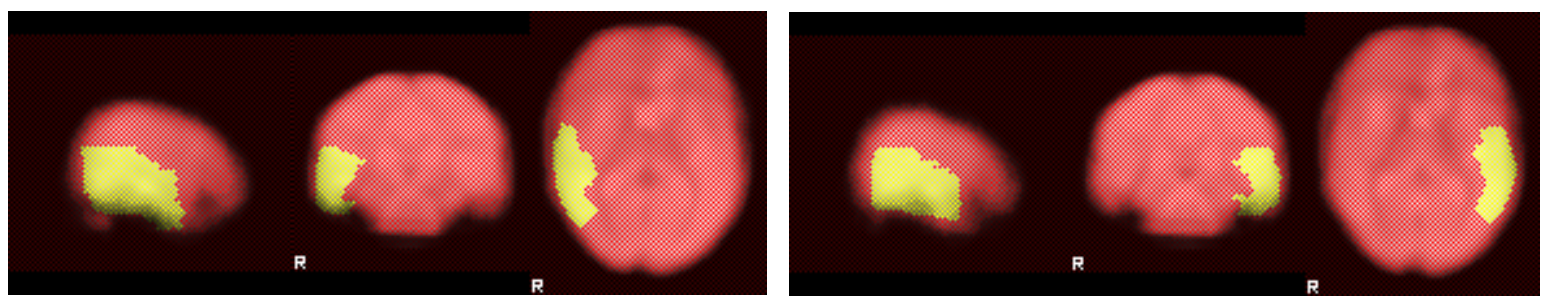

Figure A-3 Left and right temporal VOIs 
Figure A-4(36)
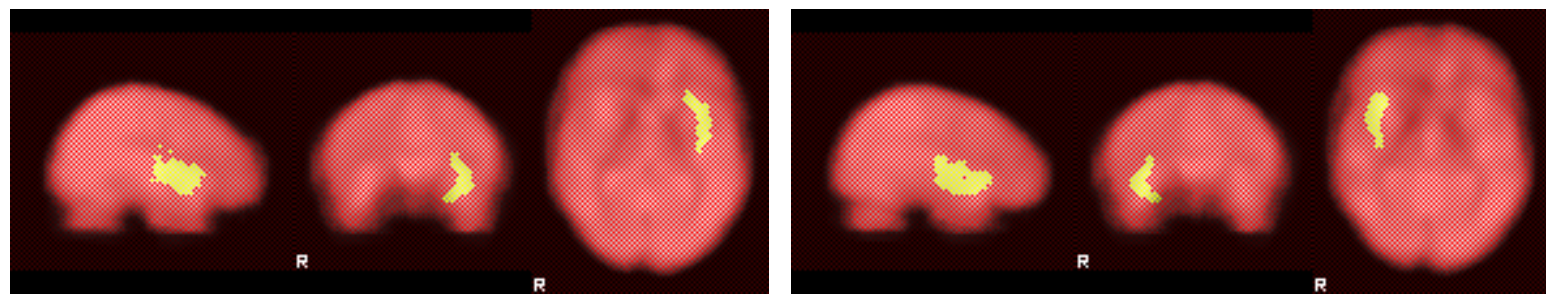

Figure A-4 Left and right insula VOIs

Figure A-5(37)
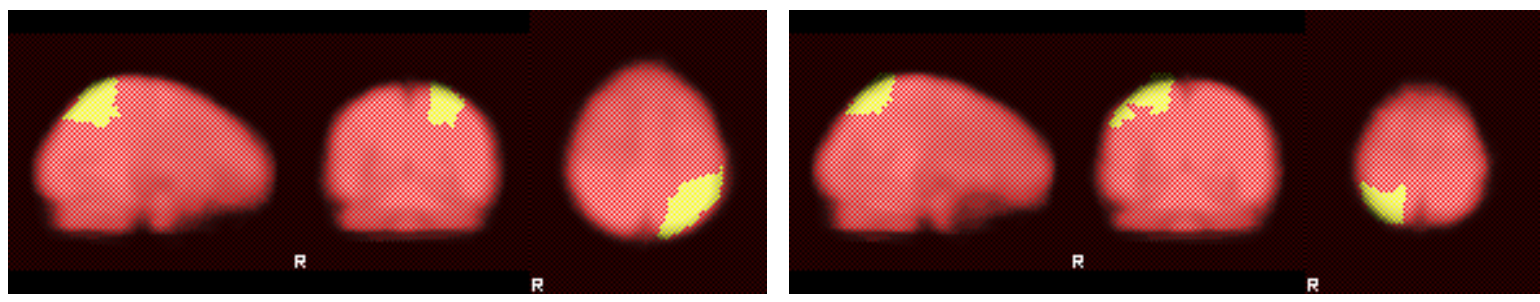

Figure A-5 Left and right parietal VOIs

Figure A-6(38)
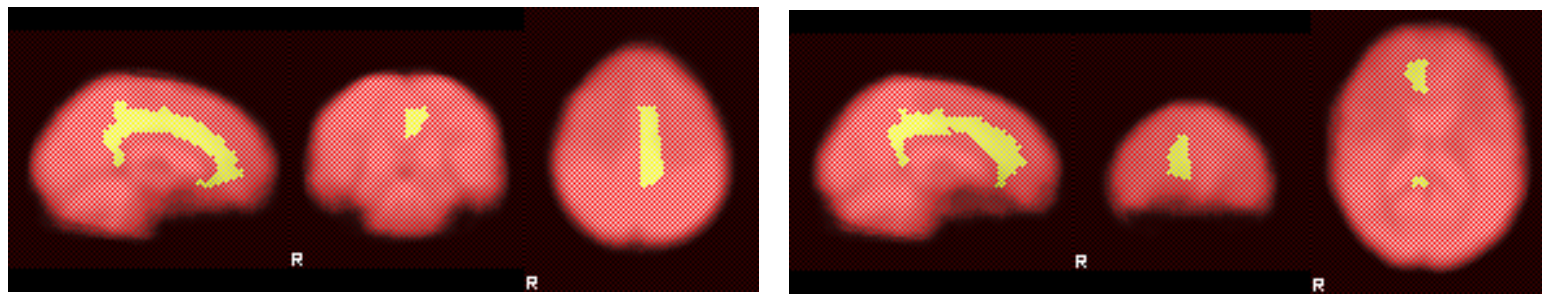

Figure A-6 Left and right cingulate VOIs 
Figure A-7(39)
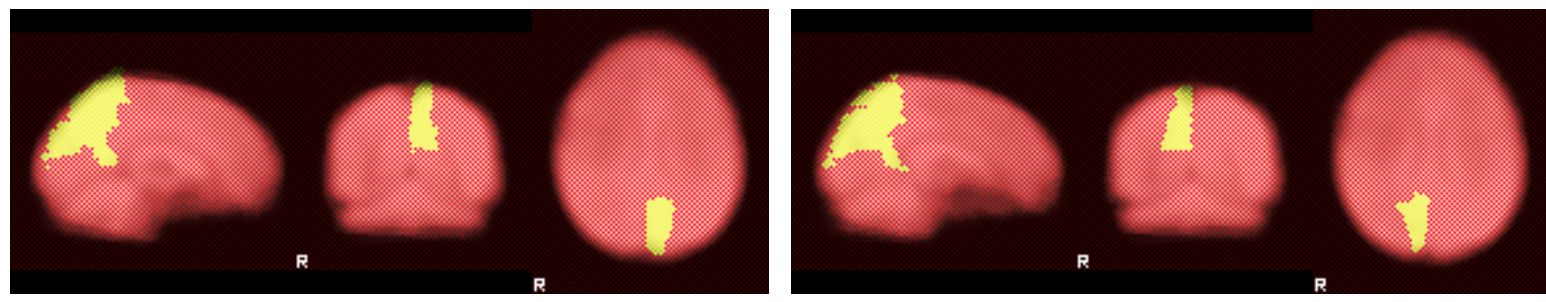

Figure A-7 Left and right cuneus VOIs

Figure A-8(40)
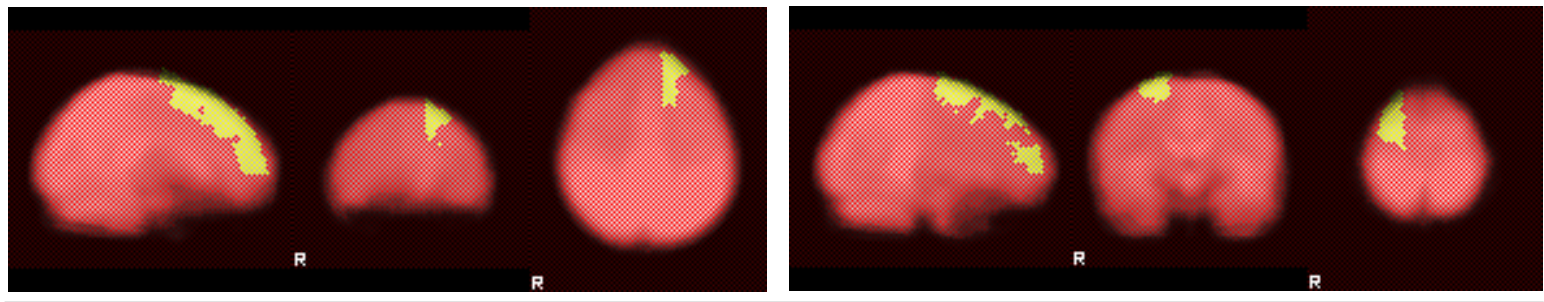

Figure A-8 Left and right superior frontal sulcus/gyrus VOIs

Figure A-9(41)
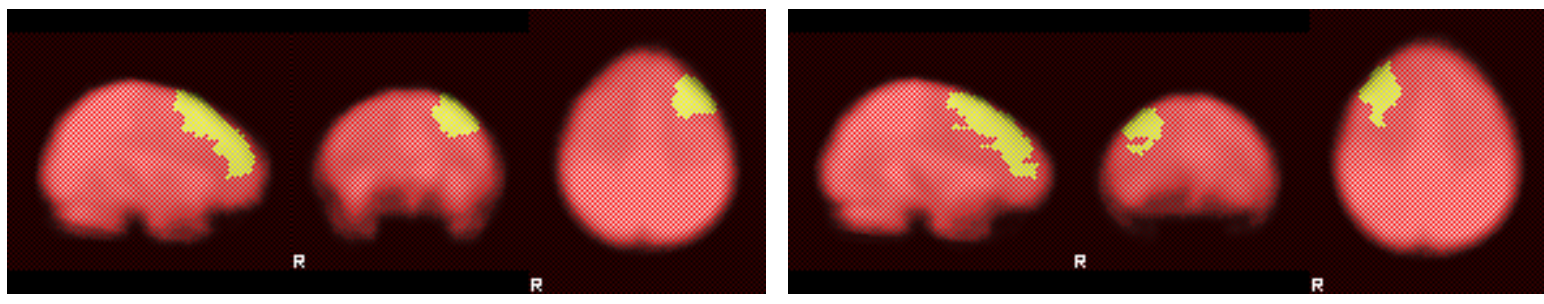

Figure A-9 Left and right middle frontal gyrus VOIs 
Figure A-10(42)
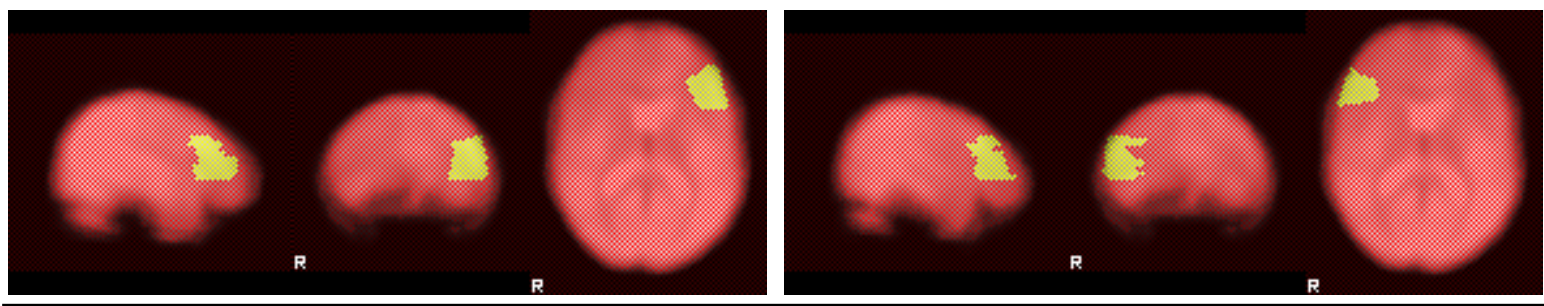

Figure A-10 Left and right pars triangularis VOIs

Figure A-11(43)
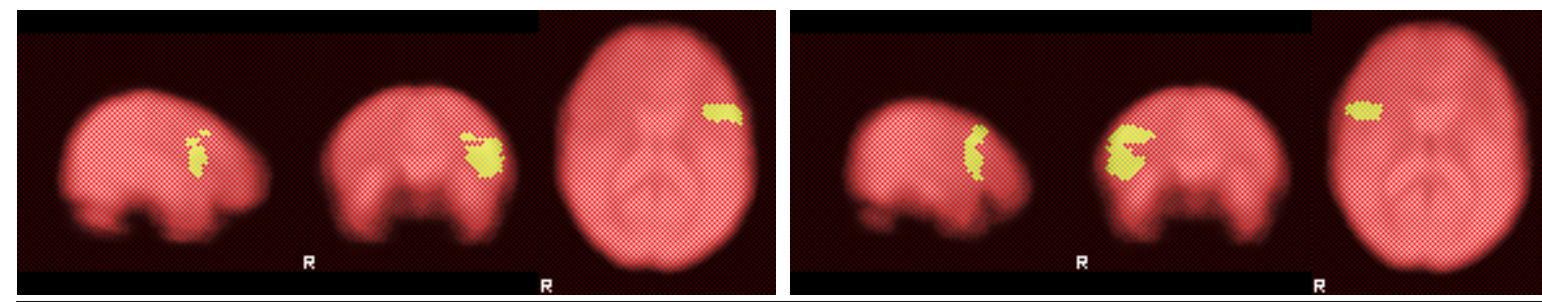

Figure A-11 Left and right pars opercularis VOIs

Figure A-12(44)
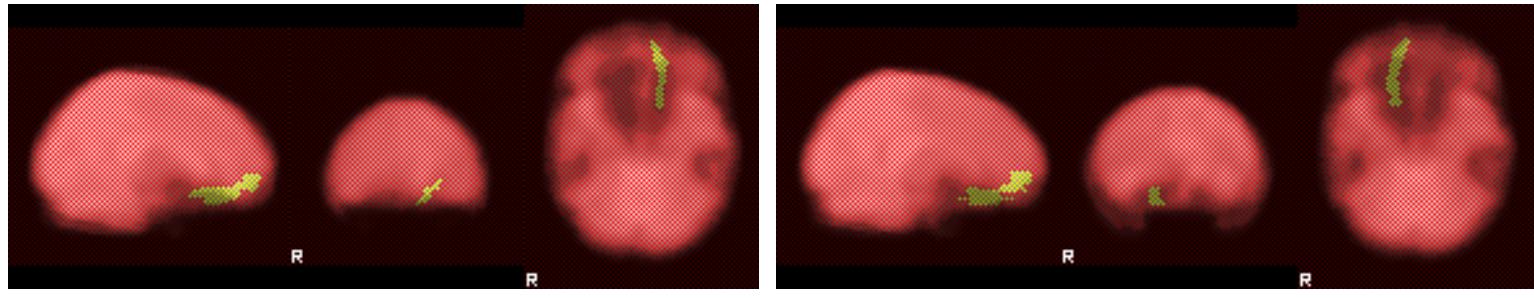

Figure A-12 Left and right supraorbital sulcus VOIs 
Figure A-13(45)
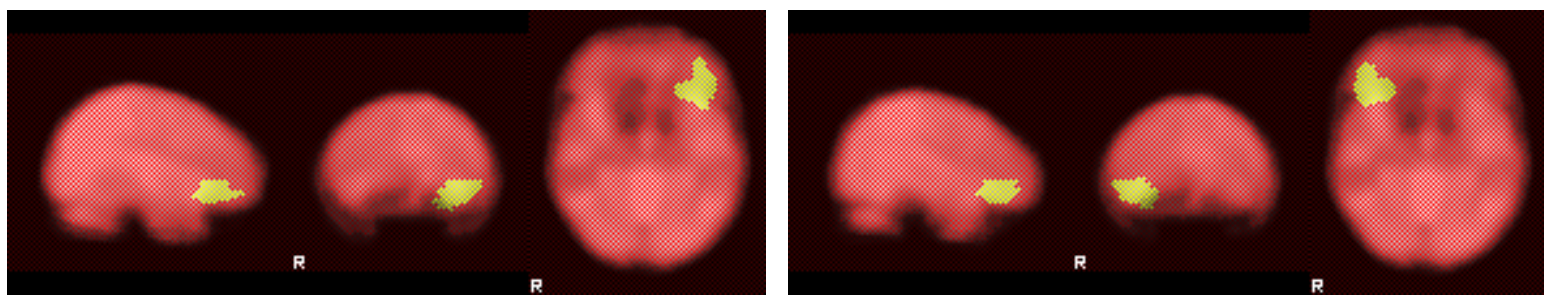

Figure A-13 Left and right pars orbitalis VOIs

Figure A-14(46)

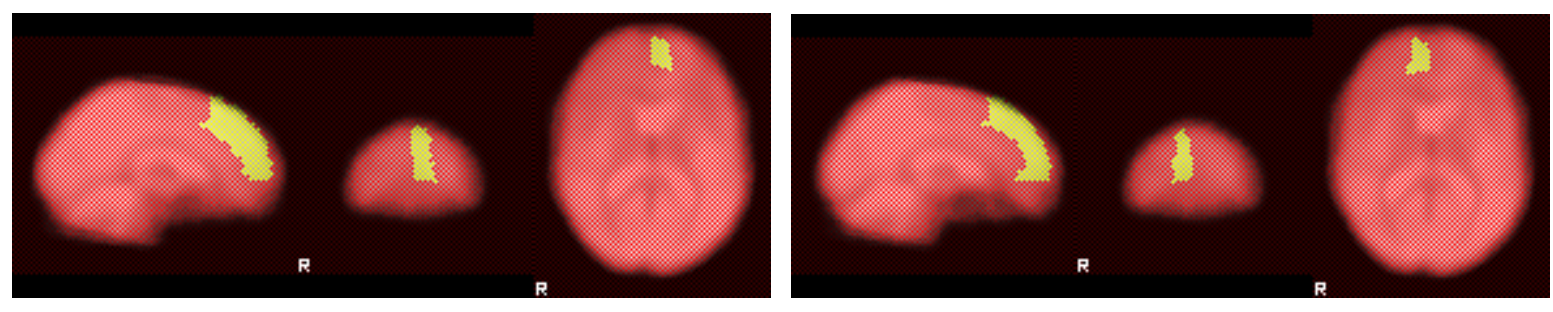

Figure A-14 Left and right medial superior frontal gyrus VOIs 


\section{A.3 Worsley Correction Calculation}

When searching in a particular VOI for activation, the small volume correction takes into account the shape and size of the particular region of interest. Unlike SPM, which allows the user to incorporate small volume corrections into the data analysis which is then calculated by SPM, FSL requires user calculations based on values from the FSL analysis.

The FSL tool Featquery is used to look at analyzed data within a mask or defined coordinate. Masks can be imported from other sources (for example, PickAtlas regions of interest) or using FSL-defined masks. Featquery then calculates statistics on the data within the chosen area: min, mean, median, max, as well as values at $10 \%$ and $90 \%$ of the collective distribution function. Also reported are the coordinates for the maximum image voxel within the mask in both native and standard (MNI) space. An example of this can be seen in the figure below:

Figure A-15

\begin{tabular}{|c|c|c|c|c|c|c|c|c|c|c|c|c|c|c|}
\hline \multirow{3}{*}{\begin{tabular}{|l} 
stats image \\
\\
thresh_zstat1 \\
\end{tabular}} & \multirow{3}{*}{\begin{tabular}{|l|} 
\\
\\
\\
\\
13
\end{tabular}} & \multicolumn{6}{|c|}{ image statistics } & \multicolumn{7}{|c|}{ position of max } \\
\hline & & \multirow{2}{*}{$\begin{array}{l}\min \\
0\end{array}$} & \multirow{2}{*}{$\frac{10 \%}{0}$} & \multirow{2}{*}{\begin{tabular}{|c} 
mean \\
0.009186
\end{tabular}} & \multirow{2}{*}{\begin{tabular}{|l|} 
median \\
0
\end{tabular}} & \multirow{2}{*}{$\begin{array}{l}90 \% \\
0\end{array}$} & \multirow{2}{*}{\begin{tabular}{|l|}
$\max$ \\
1.99
\end{tabular}} & \multicolumn{3}{|c|}{$\begin{array}{c}\text { vox (FMRI } \\
\text { space) }\end{array}$} & \multicolumn{3}{|c|}{$\begin{array}{l}\text { mm (standard } \\
\text { space) }\end{array}$} & \multirow{2}{*}{\begin{tabular}{|l|}
\multicolumn{1}{|c|}{$\begin{array}{c}\text { max } \\
\text { voxel } \\
\text { atlas } \\
\text { results }\end{array}$} \\
$13 \%$ \\
Temporal \\
Lobe
\end{tabular}} \\
\hline & & & & & & & & 15 & 69 & 25 & 60.0 & 12.0 & -22.0 & \\
\hline thresh_zstat2 & 10 & 0 & 0 & 0.007523 & 0 & 0 & 2.286 & 29 & 74 & 20 & 32.0 & 22.0 & -32.0 & $\begin{array}{l}64 \% \\
\text { Temporal } \\
\text { Lobe, 1\% } \\
\text { Frontal } \\
\text { Lobe }\end{array}$ \\
\hline thresh_zstat3 & 4 & 0 & 0 & 0.002669 & 0 & 0 & 1.738 & 20 & 69 & 21 & 50.0 & 12.0 & -30.0 & $\begin{array}{l}83 \% \\
\text { Temporal } \\
\text { Lobe }\end{array}$ \\
\hline thresh_zstat4 & 11 & 0 & 0 & 0.008181 & 0 & 0 & 2.052 & 20 & 70 & 20 & 50.0 & 14.0 & -32.0 & $\begin{array}{l}85 \% \\
\text { Temporal } \\
\text { Lobe }\end{array}$ \\
\hline
\end{tabular}

The first column, stats image, is the contrast set up by the user in the FSL Feat experimental design section. In this example, thresh-zstat1 refers to as fear, 2 is surprise, 3 is happy, and 4 is 
motion control (all versus baseline). This can be designated in whatever manner the user desires and can be reviewed in the set-up file.

For calculating a Worsley-type correction, a spatial smoothness value - or resel value - is needed. This is obtained from the FSL data analysis output folder. Then, the number of voxels in each mask is calculated using the command $f$ slstats <insert mask image name here > $-V$. Of the two numbers that are given, the second is the total volume number of the mask. The resel number is then divided into the mask voxel number, the result of this calculation is used in the command ptoz $0.05-g$ <calculated value >. This final operation will give a $\mathrm{z}$-value that corresponds to the FWE correction for the given volume. The corrected value can be compared to max z-values within the region of interest (see bold values in figure above), any z-value above the calculated/corrected $\mathrm{z}$-value is considered significant at a $\mathrm{p}<0.05$ (corrected) level. This process is then repeated for each region of interest as the mask voxel number will change for each individual mask. 


\section{A.4 Forms}

The following two forms were given to subjects to fill out before participation in experiments. The first form (Edinburgh Handedness) was used for all behavioral and fMRI experiments. This determined whether the subject was left- or right-handed. The second form (MRI Examination Information and Education Form) ensured that subjects were qualified to participate in the fMRI studies; any subjects taking psychotropic medications (i.e. for depression, attention deficit disorder, etc...), who had metal or unsafe implants in or on their body, and/or who reported being pregnant were disqualified from the study. Because these forms contained sensitive information, they were locked in a filing cabinet upon their completion. 


\section{Center for Advanced Imaging, West Virginia University}

\section{Edinburgh Handedness Inventory}

Please indicate your preferences in the use of hands in the following activities by putting a check in the appropriate column. If a preference is so strong that you would never try to use the other hand, unless absolutely forced to, put 2 checks. If in any case you are really indifferent put and a check in both left and right columns.

Some of the activities listed below require the use of both hands. In these cases the part of the task, or object, for which hand preference is wanted is indicated in brackets.

Please try and answer all of the questions, and only leave a blank if you have no experience at all with the object or task.

\begin{tabular}{|l|l|l|}
\hline \multicolumn{1}{|c|}{ ACTIVITY } & LEFT & RIGHT \\
\hline 1. Writing & $\square \square$ & $\square \square$ \\
\hline 2. Drawing & $\square \square$ & $\square \square$ \\
\hline 3. Throwing & $\square \square$ & $\square \square$ \\
\hline 4. Scissors & $\square \square$ & $\square \square$ \\
\hline 5. Toothbrush & $\square \square$ & $\square \square$ \\
\hline 6. Knife (without fork) & $\square \square$ & $\square \square$ \\
\hline 7. Spoon & $\square \square$ & $\square \square$ \\
\hline 8. Broom (upper hand) & $\square \square$ & $\square \square$ \\
\hline 9. Striking Match (match) & $\square \square$ & $\square \square$ \\
\hline 10. Opening box (lid) & $\square \square$ & $\square \square$ \\
\hline \multicolumn{1}{|c|}{ (XOTAL in each column) } & $\square$ & $\square$ \\
\hline
\end{tabular}

Percent Handedness Score $=($ Total Right - Total Left $) \times 5=$ 


\section{CENTER FOR ADVANCED IMAGING MRI EXAMINATION INFORMATION AND EDCUATION FORM}

TO BE COMPLETED BY THE PARTICIPANT

ATTENTION: It is important that you complete this form entirely

Do you have a history of diabetes?

Do you have a history of kidney disease?

List all surgeries with dates, if known:

List all current medication:

Please list all allergies:
Yes
$\square$ No
Yes
No 
Other (please specify):

Any other information that you feel may be pertinent to your study, please list below:

The above information is complete and correct. This sheet has been fully explained to me and I have had the opportunity to express all concerns and questions about my exam.

Participant Signature

Date 


\section{A.5 Determining Optimal Scanning Parameters}

Because the amygdala is located close to an air-tissue interface and is thus prone to MR signal dropout, measures were taken to ensure that optimal amygdala scanning parameters were selected. This was done prior to the commencement of Experiment 2 (Chapter 4).

The Whalen lab, (see Whalen et al., 1998; 2004) which routinely images the amygdala, used an oblique axial imaging protocol that focused only on the amygdala region of the brain and utilized small slice thicknesses. However, this type of acquisition was ruled out early on for the studies presented here as there was an interest in obtaining data from brain regions outside of the amygdala. In addition, we did not find that it reliably captured signal from the amygdala when tested out on our scanner. In order to establish our own protocol for imaging the amygdala, scanning parameters such as TR (time of repeat), TE (time of echo), slice thickness, slice plane, type of pulse sequence, and frequency were varied systematically to produce different combinations of imaging parameters. Comparisons of the functional data resulting from the different combinations were examined to determine which gave a reliable signal from both the left and right amygdala and also allowed for whole brain coverage. The final parameters that comprise our protocol are outlined in Chapter 4, Section 4.3.5.

After data had been collected and reconstructed, functional images were overlaid on anatomical images to ensure that there was adequate signal in and around the amygdala. This was done for each fMRI subject's data before preprocessing steps were initiated. 


\section{References}

Adams RB, Kleck RE (2003) Perceived gaze direction and the processing of facial displays of emotion. Psychol Sci 14: 644-47.

Adolphs R, Tranel D, Damasio H, Damasio A (1994) Impaired recognition of emotion in facial expressions following bilateral damage to the human amygdala. Nature 372: 669-72.

Adolphs R, Tranel D, Damasio AR (1998) The human amygdala in social judgment. Nature 393: 470-74.

Adolphs R, Tranel D (1999) Preferences for visual stimuli following amygdala damage. J Cogn Neurosci 11: 610-16.

Adolphs R, Tranel D, Hamann S, Young AW, Calder A.J, Phelps EA, Anderson A, Lee GP, Damasio AR (1999) Recognition of facial emotion in nine individuals with bilateral amygdala damage. Neuropsychologia 37: 1111-7.

Adolphs R (2002) Neural systems for recognizing emotion. Curr Opin Neurobiol 12: 169-77.

Adolphs R (2003a) Cognitive neuroscience of human social behavior. Nat Rev Neurosci 4: 16578.

Adolphs R (2003b) Is the human amygdala specialized for processing social information? Ann NY Acad Sci 985: 326-40.

Adolphs R, Tranel D (2003) Amygdala damage impairs recognition from scenes only when they contain facial expressions. Neuropsychologia 41: 1281-9.

Adolphs R, Gosselin F, Buchanan TW, Tranel D, Schyns P, Damasio AR (2005) A mechanism for impaired fear recognition after amygdala damage. Nature 433: 68-72.

Adolphs R, Spezio M (2006) Role of the amygdala in processing visual social stimuli. Prog Brain Res 156: 363-78.

Adolphs R (2008) Fear, faces, and the human amygdala. Curr Opin Neurobiol 18: 166-72.

Alheid, GF (2003) Extended amygdala and basal forebrain. Ann N Y Acad Sci 985: 185-205.

Allison T, Ginter H, McCarthy G, Nobre AC, Puce A, Luby M, Spencer DD (1994) Face recognition in human extrastriate cortex. J Neurophysiol 71: 821-25. 
Allison T, Puce A, Spencer DD, McCarthy G (1999) Electrophysiological studies of human face perception. I: Potentials generated in occipitotemporal cortex by face and non-face stimuli. Cereb Cortex 9: 415-30.

Amaral DG, Insausti R (1992) Retrograde transport of D-[3H]-aspartate injected into the monkey amygdaloid complex. Exp Brain Res 88: 375-88.

Amaral DG, Behniea H, Kelly JL (2003) Topographic organization of projections from the amygdala in the visual cortex of the macaque monkey. Neuroscience 118: 1099-120.

Andreasen NC, O'Leary DS, Arndt S, Cizadlo T, Hurtig R, Rezai K, Watkins GL, Ponto LB, Hichwa RD (1996) Neural substrates of facial recognition. J Neuropsychiatry Clin Neurosci 8: 139-46.

Andrews TJ, Schluppeck D (2004) Neural responses to Mooney images reveal a modular representation of faces in human visual cortex. Neuroimage 21: 91-8.

Anderson AK, Phelps EA (2001) Lesions of the human amygdala impair enhanced perception of emotionally salient events. Nature 411: 305-9.

Anderson AK, Christoff K, Panitz D, De Rosa E, Gabrieli JD (2003) Neural correlates of the automatic processing of threat facial signals. J Neurosci 23: 5627-33.

Armony JL, Servan-Schreiber D, Romanski LM, Cohen JD, LeDoux JE (1997) Stimulus generalization of fear responses: effects of auditory cortex lesions in a computational model and in rats. Cereb Cortex 7: 157-65.

Baas D, Aleman A, Kahn RS (2004) Lateralization of amygdala activation: a systematic review of functional neuroimaging studies. Brain Res Brain Res Rev 45: 96-103.

Bach DR. Schäechinger J, Neuhoff JG, Esposito F, Di Salle F, Lehmann C, Herdener M, Scheffler K, Seifritz E (2008) Rising sound intensity: an intrinsic warning cue activating the amygdala. Cereb Cortex 18: 145-50.

Baron-Cohen S, Bolton, P (1993) Autism: the facts. Oxford: Oxford University Press.

Baron-Cohen S, Ring HA, Bullmore ET, Wheelwright S, Ashwin C, Williams SC (2000)

The amygdala theory of autism. Neurosci Bibehav Rev 24: 355-64.

Barton JJ (2003) Disorders of face processing and recognition. Neurol Clin 21: 521-48.

Barton RA Aggleton JP (2000) Primate evolution and the amygdala. In: Aggleton JP (Ed.) The amygdala: a functional analysis. (pp 479-508) Oxford: Oxford University Press.

Batki A, Baron-Cohen S, Wheelwright S, Connellan J, Ahluwalia J (2000) Is there an innate gaze module? Evidence from human neonates. Infant Behav Dev 23: 223-29. 
Baxter MG, Murray EA, (2002) The amygdala and reward. Nat Rev Neurosci 3: 563-73.

Bechara A, Tranel D, Damasio H, Adolphs R, Rockland C, Damasio AR (1995) Double dissociation of conditioning and declarative knowledge relative to the amygdala and hippocampus in humans. Science 269: 1115-8.

Benowitz LI, Bear DM, Rosenthal R, Mesulam MM, Zaidel E, Sperry RW (1983) Hemispheric specialization in nonverbal communication. Cortex 19: 5-11.

Bentin S, Allison T, Puce A, Perez E, McCarthy G (1996) Electrophysiological studies of face perception in humans. J Cogn Neurosci 8: 551-65.

Bentin S, Deouell LY (2000) Structural encoding and identification in face processing: ERP evidence for separate mechanisms. Cog Neuropsych 17: 35-54.

Benuzzi F, Meletti S, Zamboni G, Calandra-Buonaura G, Serafini M, Lui F, Baraldi P, Rubboli G, Tassinari CA, Nichelli P (2004) Impaired fear processing in right mesial temporal sclerosis: an fMRI study. Brain Res Bull 63: 269-81.

Benuzzi F, Matteo P, Meletti S, Lui F, Serafini M, Baraldi P, Nichelli P (2007) Processing the socially relevant parts of faces. Brain Res Bull 74: 344-56.

Bichot NP, Schall JD (1999) Effects of similarity and history on neural mechanisms of visual selection. Nat Neurosci 2: 549-554.

Bisti S, Sireteanu RC (1976) Sensitivity to spatial frequency and contrast of visual cells in the cat superior colliculus. Vision Res 16: 247-51.

Blair RJ, Morris JS, Frith CD, Perrett DI, Dolan RJ (1999) Dissociable neural responses to facial expressions of sadness and anger. Brain 122: 883-893.

Bond NW, Siddle DAT (1996) The preparedness account of social phobia: some data and alternative explanations. In: Rapee RM (Ed.) Current controversies in the anxiety disorders. (pp 291-316) London: Guilford Press.

Bonda E, Petrides M, Ostry D, Evans A (1996) Specific involvement of human parietal systems and amygdala in the perception of biological motion. $J$ Neurosci 16: 3737-44.

Bracewell RN (1986) The Fourier transform and its applications. New York: McGraw-Hill.

Breen N, Caine D, Coltheart M (2000) Models of face recognition and delusional misidentification: A critical review. Cog Neuropsychology 17: 55-71. 
Breiter HC, Etcoff NL, Whalen PJ, Kennedy WA, Rauch SL, Buckner RL, Strauss MM, Hyman SE, Rosen BR (1996) Response and habituation of the human amygdala during visual processing of facial expression. Neuron 17: 875-87.

Bruce V, Young AW (1986) Understanding face recognition. Br J Psychol 77: 305-27.

Buckner RL, Bandettini PA, O’Craven KM, Savoy RL, Petersen SE, Raichle ME, Rosen BR (1996) Detection of cortical activation during averaged single trials of a cognitive task using functional magnetic resonance imaging. Proc Natl Acad Sci U S A 93: 14878-83.

Cabeza R, Kato T (2000) Features are also important: contributions of featural and configural processing to face recognition. Pyscho Sci 11: 429-33.

Cahill L, Haier RJ, White NS, Fallon J, Kilpatrick L, Lawrence C, Potkin SG, Alkire MT (2001) Sex-related difference in amygdala activity during emotionally influenced memory storage. Neurobiol Learn Mem 75: 1-9.

Caldara R, Schyns P, Mayer E, Smith ML, Gosselin F, Rossion B (2005) Does prosopagnosia take the eyes out of face representations? Evidence for a defect in representing diagnostic facial information following brain damage. J Cogn Neurosci 17: 1652-66.

Calder AJ, Keane J, Cole J, Campbell R, Young AW (2000) Facial expression recognition by people with Möbius syndrome. Cog Neuropsychology 17: 73-87.

Calder AJ, Lawrence AD, Young AW (2001) Neuropsychology of fear and loathing. Nat Rev Neurosci 2: 352- 63 .

Calvert GA, Bullmore ET, Brammer MJ, Campbell R, Williams SC, McGuire PK, Woodruff PW, Iversen SD, David AS (1997) Activation of auditory cortex during silent lipreading. Science 276: 593-96.

Calvert GA, Campbell R (2003) Reading speech from still and moving faces: the neural substrates of visible speech. J Cogn Neurosci 15: 57-70.

Calvo MG, Nummenmaa L (2008) Detection of emotional faces: salient physical features guide effective visual search. J Exp Psychol Gen 137: 471-94.

Chao LL, Haxby JV, Martin A (1999) Attribute-based neural substrates in temporal cortex for perceiving and knowing about objects. Nat Neurosci 2: 913-19.

Clark VP, Keil K, Maisog JM, Courtney S, Ungerleider LG, Haxby JV (1996) Functional magnetic resonance imaging of human visual cortex during face matching: a comparison with positron emission tomography. Neuroimage 4: 1-15.

Corballis MC (1995) Visual integration in the split brain. Neuropsychologia 33: 937-59. 
Corbetta M, Miezin FM, Shulman GL, Petersen SE (1993) A PET study of visuospatial attention. J Neurosci 13: 1202-26.

Corbetta M, Shulman GL (2002) Control of goal-directed and stimulus-driven attention in the brain. Nat Rev Neurosci 3: 201-15.

Critchley HD, Daly EM, Bullmore ET, Williams SC, Van Amelsvoort T, Robertson DM, Rowe A, Phillips M, McAlonan G, Howlin P, Murphy DG (2000) The functional neuroanatomy of social behaviour: Changes in cerebral blood flow when people with autistic disorder process facial expressions. Brain 123: 2203-12.

Critchley HD, Rotshtein P, Nagai Y, O’Doherty J, Mathias CJ, Dolan RJ (2005) Activity in the human brain predicting differential heart rate response to emotional facial expressions. Neuroimage 24: 751-62.

Cunningham WA, Zelazo PD (2007) Attitudes and evaluations: a social cognitive neuroscience perspective. Trends Cogn Sci 11: 97-104.

Dalton KM, Nacewicz BM, Johnstone T, Schaefer HS, Gernsbacher MA, Goldsmith HH, Alexander AL, Davidson RJ (2005) Gaze fixation and the neural circuitry of face processing in autism. Nat Neurosci 8: 519-26.

Darwin C (1872) The expression of the emotions of man and animals. ( $3^{\text {rd }}$ ed.) Oxford: Oxford University Press.

David AS, Brierley B, Shaw P (2002) Measuring amygdala volume. Brit J Psych 181: 255-56.

Davidson RJ, Irwin W (1999) The functional neuroanatomy of emotion and affective style. Trends in Cog Neurosci 3: 11-21.

Davis M, Whalen PJ (2001) The amygdala: vigilance and emotion. Mol Psychiatry 6: 13-34.

Decety J, Grèzes J (1999) Neural mechanisms subserving the perception of human actions. Trends Cogn Sci 3: 172-78.

de Gelder B, Vroomen J, Pourtois G, Weiskrantz L (1999) Non-conscious recognition of affect in the absence of striate cortex. Neuroreport 10: 3759-63.

de Gelder B, Frissen I, Barton J, Hadjikhani N (2003) A modulatory role for facial expressions in prosopagnosia. Proc Natl Acad Sci U S A 100: 13105-10.

de Gelder B, Snyder J, Greve D, Gerard G, Hadjikhani N (2004) Fear fosters flight: a mechanism for fear contagion when perceiving emotion expressed by a whole body. Proc Natl Acad Sci U S A 101: 16701-6. 
Demos KE, Kelley WM, Ryan SL, Davis FC, Whalen PJ (2008) Human amygdala sensitivity to the pupil size of others. Cereb Cortex 18: 2729-34.

Driver J, Davis G, Ricciardelli P, Kidd P, Maxwell E, Baron-Cohen S (1999) Gaze perception triggers reflexive visuospatial orienting. Vis Cogn 6: 509-40.

Ekman P, Sorenson ER, Friesen WV (1969) Pan-cultural elements in facial displays on emotions. Science 164: 86-8.

Ekman P (1970) Universal facial expressions of emotion. California Mental Health Research Digest 8: 151-58.

Ekman P, Friesen WV (1976) Measuring facial movement. Environ Pysch Nonverb Behav 1: 5675.

Ellsworth PC (1975) Direct gaze as a social stimulus: the example of aggression. In: Pliner P, Krames L, Alloway T (Eds.) Nonverbal communication of aggression. New York: Plenum Press.

Emery NJ (2000) The eyes have it: the neuroethology, function and evolution of social gaze. Neurosci Biobehav Rev 24: 581-604.

Esteves F, Öhman A (1993) Masking the face: recognition of emotional facial expressions as a function of the parameters of backward masking. Scand J Psychol 34 :1-18.

Etkin A, Wager TD (2007) A meta-analysis of emotional processing in PTSD, social anxiety disorder, and specific phobia. Am J Psychiatry 164: 1476-88.

Fanselow MS, LeDoux JE (1999) Why we think plasticity underlying Pavlovian fear conditioning occurs in the basolateral amygdala. Neuron 23: 229-32.

Farroni T, Csibra G, Simion G, Johnson MH (2002) Eye contact detection in humans from birth. Proc Natl Acad Sci U S A 99: 9602-5.

Fischer H, Wright CI, Whalen PJ, McInerney SC, Shin LM, Rauch SL (2003) Brain habituation during repeated exposure to fearful and neutral faces: a functional MRI study. Brain Res Bull 59: 387-92.

Fox E, Lester V, Russo R, Bowles RJ, Pichler A, Dutton K (2000) Facial expressions of emotion: are angry faces detected more efficiently. Cogn Emot 14: 61-92.

Fox E, Damjanovic L (2006) The eyes are sufficient to produce a threat superiority effect. Emotion 6: 534-9.

Freese JL, Amaral DG (2005) The organization of projections from the amygdala to visual cortical areas TE and V1 in the macaque monkey. J Comp Neurol 486: 295-317. 
Frischen A, Bayliss AP, Tipper SP (2007) Gaze cueing of attention: visual attention, social cognition and individual differences. Psychol Bull 133: 694-724.

Friston KJ, Ashburner J, Frith CD, Poline JB, Heather JD, Frackowiak RSJ (1995) Spatial registration and normalization of images. Hum Brain Mapp 2: 165-89.

Friston KJ, Williams SCR, Howard R, Frackowiak RSJ, Turner R (1996) Movement-related effects in fMRI time series. Magn Reson Med 35: 346-55.

Friston KJ, Zarahn E, Josephs O, Henson RN, Dale AM (1999) Stochastic designs in eventrelated fMRI. Neuroimage 10: 607-19.

Gamer M, Büchel C (2009) Amygdala activation predicts gaze toward fearful eyes. J Neurosci 29: 9123-6.

Gauthier I, Tarr MJ, Moylan J, Anderson AW, Skudlarski P, Gore JC (2000) Does visual subordinate-level categorization engage the functionally-defined fusiform face area? $\mathrm{Cog}$ Neuropsychology 17: 125-42.

George N, Dolan RJ, Fink GR, Baylis GC, Russell C, Driver J (1999) Contrast polarity and face recognition in the human fusiform gyrus. Nat Neurosci 2: 574-80.

George N, Driver J, Dolan RJ (2001) Seen gaze-direction modulates fusiform activity and its coupling with other brain areas during face processing. Neuroimage 13: 1102-12.

Gilga T, Csibra G (2007) Seeing the face through the eyes: a developmental perspective on face expertise. Prog Brain Res 164: 323-39.

Glascher J, Adolphs R (2003) Processing of the arousal of subliminal and supraliminal emotional stimuli by the human amygdala. J Neurosci 23: 10274-82.

Gloor P (1997) The temporal lobe and limbic system. New York: Oxford University Press.

Glover GH, Law CS (2001) Spiral-in/out BOLD fMRI for increased SNR and reduced suspectibility artifacts. Magn Reson Med 46: 515-22.

Goldstein JM, Seidman LJ, Horton NJ, Makris N, Kennedy DN, Caviness VS, Faraone SV, Tsuang MT (2001) Normal sexual dimorphism of the adult human brain assessed by in vivo magnetic resonance imaging. Cereb Cortex 11: 490-97.

Gottilieb JP, Kusunoki M, Goldberg ME (1998) The representation of visual salience in monkey parietal cortex. Nature 391: 481-4.

Grady CL, McIntosh AR, Horowitz B, Rapoport SI (2000) Age-related changes in the neural correlates of degraded and non-degraded face processing. Cog Neuropsychology 17: 165-86. 
Grandin T (1996) Thinking in pictures and other reports from my life with autism. New York: Vintage.

Grelotti DJ, Klin AJ, Gauthier I, Skudlarski P, Cohen DJ, Gore JC, Volkmar FR, Schultz RT (2005) fMRI activation of the fusiform gyrus and amygdala to cartoon characters but not to faces in a boy with autism. Neuropsychologia 43: 373-85.

Grill-Spector K, Knouf N, Kanwisher N (2004) The fusiform face area subserves face perception, not generic within-category identification. Nat Neurosci 7: 555-62.

Grossman E, Donnelly M, Price R, Pickens D, Morgan V, Neighbor G, Blake R (2000) Brain areas involved in perception of biological motion. J Cogn Neurosci 12: 711-20.

Hadjikhani N, de Gelder B (2003) Seeing fearful body expressions activates the fusiform cortex and amygdala. Curr Biol 13: 2201-05.

Hansen CH, Hansen RD (1988) Finding the face in the crowd: an anger superiority effect. J Pers Soc Pyschol 54: 914-24.

Hardee JE, Thompson JC, Puce A (2008) The left amygdala knows fear: laterality in the amygdala response to fearful eyes. Soc Cogn Affect Neurosci 3: 47-54.

Haxby JV, Horwitz B, Ungerleider LG, Maisog JM, Pietrini P, Grady CL (1994) The functional organization of human extrastriate cortex: a PET-rCBF study of selective attention to faces and locations. J Neuroscience 14: 6336-53.

Haxby JV, Ungerleider LG, Hortwitz B, Maisog JM, Rapoport SI, Grady CL (1996) Face encoding and recognition in the human brain. Proc Natl Acad Sci U S A 93: 922-7.

Haxby JV, Hoffman EA, Gobbini MI (2000) The distributed human neural system for face perception. Trends Cogn Sci 4: 223-33.

Haxby JV, Gobbini MI, Furey ML, Ishai A, Schouten JL, Pietrini P (2001) Distributed and overlapping representations of faces and objects in ventral temporal cortex. Science 293:242530 .

Haxby JV, Hoffman EA, Gobbini MI (2002) Human neural systems for face recognition and social communication. Biol Psychiatry 51: 59-67.

Hennenlotter A, Schroeder U (2006) Partly dissociable neural substrates for recognizing basic emotions: a critical review. Prog Brain Res 156: 443-56.

Herpertz SC, Dietrich TM, Wenning B, Krings T, Erberich SG, Willmes K, Thron A, Sass H (2001) Evidence of abnormal amygdala functioning in borderline personality disorder: a functional MRI study. Biol Psychiatry 50: 292-8. 
Herry C, Bach DR, Esposito F, Di Salle F, Perrig WJ, Scheffler K, Luthi A, Seifritz E (2007) Processing of temporal unpredictability in human and animal amygdala. J Neurosci 27: 5958-66.

Hoffman EA, Haxby JV (2000) Distinct representations of eye gaze and identity in the distributed human neural system for face perception. Nat Neurosci 3: 80-84.

Holland PC, Gallagher M (2004) Amygdala-frontal interactions and reward expectancy. Curr Opin Neurobiol 14: 148-55.

Holmes A, Vuilleumier P, Eimer M (2003) The processing of emotional facial expression is gated by spatial attention: evidence from event-related brain potentials. Brain Res Cogn Brain Res 16: 174-84.

Hooker CI, Paller KA, Gitelman DR, Parrish TB, Mesulam MM, Reber PJ (2003) Brain networks for analyzing eye gaze. Brain Res Cog Brain Res 17: 406-18.

Hubl D, Bolte S, Feineis-Matthews S, Lanfermann H, Federspiel A, Strik W, Poustka R, Dierks $\mathrm{T}$ (2003) Functional imbalance of visual pathways indicates alternative face processing strategies in autism. Neurology 61: 1232-7.

Humphrey N (1976) The social function of intellect. Bateson PPG, Hinde RA (Eds.) In: Growing points in ethology. (pp 303-317) Cambridge: Cambridge University Press

Ishai A, Schmidt CF, Boesiger P (2005) Face perception is mediated by a distributed cortical network. Brain Res Bull 67: 87-93.

Itier RJ, Batty M (2009) Neural bases of eye and gaze processing: The core of social cognition. Neurosci Biobehav Rev 33: 843-63.

Iwai E, Yukie M, Suyama H, Shirakawa S (1987) Amygdalar connections with middle and inferior temporal gyri of the monkey. Neurosci Letters 83:25-9.

Jeffreys DA (1996) Evoked potential studies of face and object processing. Vis Cogn 3: 1-38.

Johnson M (2005) Subcortical face processing. Nat Rev Neurosci 6: 766-74.

Joseph RM, Tanaka J (2002) Holistic and part-based face recognition in children with autism. $J$ Child Psychol Psychiatry 44: 529-42.

Kanwisher NG, McDermott J, Chun MM (1997) The fusiform face area: a module in human extrastriate cortex specialized for face perception. J Neurosci 17: 4302-11.

Kapp BS, Whalen PJ, Supple WF, Pascoe JP (1992) Amygdaloid contributions to conditioned arousal and sensory information processing. In: Aggleton, JP (Ed.) The amygdala:

neurobiological aspects of emotion, memory, and mental dysfunction. (pp 229-32) New York: Wiley-Liss. 
Kawashima R, Sugiura M, Kato T, Nakamura A, Hatano K, Ito K, Fukuda H, Kojima S, Nakamura K (1999) The human amygdala plays an important role in gaze monitoring: A PET study. Brain 122: 779-83.

Keightley ML, Winocur G, Graham SJ, Mayberg HS, Hevenor SJ, Grady CL (2003) An fMRI study investigating cognitive modulation of brain regions associated with emotional processing of visual stimuli. Neuropsychologia 41: 585-96.

Kilgore WDS, Yurgelun-Todd D (2001) Sex differences in amygdala activation during the perception of facial affect. Neuroreport 12: 2543-7.

Kim H, Somerville LH, Johnstone T, Alexander AL, Whalen PJ (2003) Inverse amygdala and medial prefrontal cortex response to surprised faces. Neuroreport 14: 2317-22.

Kim H, Somerville LH, Johnstone T, Polis S, Alexander AL, Shin LM, Whalen PJ (2004) Contextual modulation of amygdala responsivity to surprised faces. J Cogn Neurosci 16:173045 .

Klin A, Jones W, Schultz R, Volkmar F, Cohen D (2002) Visual fixation patterns during viewing naturalistic social situations as predictors of social competence in individuals with autism. Arch Gen Psychiatry 59: 809-16.

Kluver H, Bucy PC (1939) Preliminary analysis of functions of the temporal lobes in monkeys. Arch Neuro Psychiatr 42: 979-1000.

Kobayashi H, Koshima S (1997) Unique morphology of the human eye. Nature 387: 767-8.

Kosaka H, Omori M, Murata T, Iidaka T, Yamada H, Okada T, Takahashi T, Sadato N, Itoh H, Yonekura Y, Wada Y (2002) Differential amygdala response during facial recognition in patients with schizophrenia: an fMRI study. Schizophr Res 57: 87-95.

Kourtzi Z, Kanwisher N (2002) Activation in human MT/MST by static images with implied motion. J Cogn Neurosci 12: 48-55.

Krettek PE, Price JL (1978) A description of the amygdaloid complex in the rat and cat with observations on intra-amygdaloid axonal connections. J Comp Neurol 178: 255-80.

LaBar KS, LeDoux JE, Spencer DD, Phelps EA (1995) Impaired fear conditioning following unilateral temporal lobectomy in humans. J Neurosci 15: 6846-55.

LaBar KS, LeDoux JE (1996) Partial disruption of fear conditioning in rats with unilateral amygdala damage: correspondence with unilateral temporal lobectomy in humans. Behav Neurosci 110: 991-97. 
LaBar KS, Gatenby JC, Gore JC, LeDoux JE, Phelps EA (1998) Human amygdala activation during conditioned fear acquisition and extinction: a mixed-trial fMRI study. Neuron 20: 93745 .

Lang PJ, Bradley MM, Fitzsimmons JR, Cuthbert BN, Scott JD, Moudler B, Nangia V (1998) Emotional arousal and activation of the visual cortex: an fMRI analysis. Psychophysiology 35: 199-210.

Langton ST, Bruce V (1999) Reflexive visual orienting in response to the social attention of others. Vis Cogn 6: 541-67.

Lawrie SM, Whalley HC, Job DE, Johnstone EC (2003) Structural and functional abnormalities of the amygdala in schizophrenia. Ann NY Acad Sci 985: 445-60.

LeDoux JE (1996) The emotional brain: mysterious underpinnings of emotional life. New York: Simon and Schuster.

LeDoux J (1998) Fear and the brain: where have we been, and where are we going? Biol Psychiatry 44: 1229-38.

LeDoux JE (2007) The amygdala. Curr Biol 20: R868-874.

Lehmann H, Treit D, Parent MB (2000) Amygdala lesions do not impair shock-probe avoidance retention performance. Behav Neurosci 114: 107-16.

Leppänen JM, Hietana JK, Koskinen K (2008) Differential early ERPs to fearful versus neutral facial expressions: a response to the salience of the eyes? Biol Psychol 78: 150-8.

Liberzon I, Sripada CS (2008) The functional neuroanatomy of PTSD: a critical review. Prog Brain Res 167: 151-69.

Luh KE, Rueckert LM, Levy J (1991) Perceptual asymmetries for free viewing of several types of chimeric stimuli. Brain Cogn 16: 83-103.

Maestro S, Muratori F, Cavallaro MC, Pei F, Stern D, Golse B, Palacio-Espasa F (2002) Attentional skills during the first 6 months of age in autism spectrum disorder. J Am Acad Child Adolesc Psychiatry 41: 1239-45.

Marcus DJ, Nelson CA (2001) Neural basis and development of face recognition in autism. CNS Spectrums 6: 36-59.

Marinkovic K, Trebon P, Chauvel P, Halgren E (2000) Localized face-processing by the human prefrontal cortex: Face-selective intracerebral potentials and post-lesion deficits. Cog Neuropsychology 17: 187-99. 
Markowitsch H (1998) Differential contribution of right and left amygdala to affective information processing. Behav Neurol 11: 233-44.

Marwick K, Hall J (2008) Social cognition in schizophrenia: a review of face processing. $\mathrm{Br}$ Med Bull 88: 43-58.

McCarthy G, Puce A, Gore JC, Allison T (1997) Face-specific processing in the human fusiform gyrus. J Cogn Neurosci 9: 605-10.

McDonald AJ, Mascagni F, Guo L (1996) Projections of the medial and lateral prefrontal cortices to the amygdala: a Phaseolus vulgaris leucoagglutinin study in the rat. Neuroscience 71: $55-75$.

McGaugh JL (2000) Amygdala: role in modulation of memory storage. Aggleton JP (Ed.) In: The amygdala: a functional analysis. (pp 391-424) New York: Oxford University Press.

Merigan WH, Katz LM, Maunsell JH (1991) The effects of parvocellular lateral geniculate lesions of the acquity and contrast sensitivity of macaque monkeys. J Neurosci 11: 994-1001.

Mineka S, Davidson M, Cook M, Keir R (1984) Observational conditioning of snake fear in rhesus monkeys. J Abnorm Psychol 93: 355-72.

Morris JS, Frith CD, Perrett DI, Rowland D, Young AW, Calder AJ, Dolan RJ (1996) A differential response in the human amygdala to fearful and happy facial expressions. Nature 383: $812-5$.

Morris JS, Friston KJ, Buchel C, Frith CD, Young AW, Calder AJ, Dolan RJ (1998a) A neuromodulatory role for the human amygdala in processing emotional facial expressions. Brain 121: 47-57.

Morris JS, Ohman A, Dolan RJ (1998b) Conscious and unconscious emotional learning in the human amygdala. Nature 393: 467-70.

Morris JS, Ohman A, Dolan RJ (1999) A subcortical pathway to the right amygdala mediating “unseen'” fear. Proc Natl Acad Sci U S A 96: 1680-5.

Morris JS, de Gelder B, Weiskrantz L, Dolan RJ (2001) Differential extrageniculostriate and amygdala responses to presentation of emotional faces in a cortically blind field. Brain 124: 1241-52.

Morris JS, deBonis M, Dolan RJ (2002) Human amygdala responses to fearful eyes. Neuroimage 17: 214-22.

Narumoto J, Okada T, Sadato N, Fukui K, Yonekura Y (2001) Attention to emotion modulates fMRI activity in human right superior temporal sulcus. Brain Res Cog Brain Res 12: 225-31. 
Neumann D, Spezio M, Piven J, Adolphs R (2006) Looking you in the mouth: abnormal gaze in autism resulting from impaired top-down modulation of visual attention. Soc Cogn Affect Neurosci 1: 194-202.

New AS, Hazlett EA, Buchsbaum MS, Goodman M, Mitelman SA, Newmark R, Trisdorfer R, Haznedar MM, Koenigsberg HW, Flory J, Siever LJ (2007) Amygdalaprefrontal disconnection in borderline personality disorder. Neuropsychopharmacology 32: $1629-40$.

Noesselt T, Driver J, Heinze HJ, Dolan R (2005) Assymetrical activation in the human brain during processing of fearful faces. Curr Biol 15: 424-9.

Norris CJ, Chen EE, Zhu DC, Small SL, Cacioppo JT (2004) The interaction of social and emotional processes in the brain. J Cogn Neurosci 16: 1818-29.

Nunes PM, Wenzel A, Borges KT, Porto CR, Caminha RM, de Oliveira IR (2009) Volumes of the hippocampus and amygdala in patients with borderline personality disorder: a meta-analysis. J Pers Disord 23: 333-45.

Ogawa S, Lee TM, Kay AR, Tank DW (1990) Brain magnetic resonance imaging with contrast dependent on blood oxygenation. Proc Natl Acad Sci U S A 87: 9868-72.

Öhman A (1986) Face the beast and fear the face: animal and social fears as protoptypes for evolutionary analyses of emotion. Psychophysiology 23: 123-45.

Öhman A, Lundqvist D, Esteves F (2001) The face in the crowd revisted: a threat advantage with schematic stimuli. J Pers Soc Psychol 80: 381-96.

Öhman A, Soares JJ (1993) On the automatic nature of phobic fear: conditioned electrodermal responses to masked fear-relevant stimuli. J Abnorm Pyschol 102:121-32.

Orban de Xivry JJ, Ramon M, Lefèvre P, Rossion B (2008) Reduced fixation on the upper area of personally familiar faces following acquired prosopagnosia. J Neuropsychol 2: 245-68.

Ousdal OT, Jensen J, Server A, Hariri AR, Nakstad PH, Andreassen OA (2008) The human amygdala is involved in general behavioral relevance detection: evidence from an event-related functional magnetic resonance imaging Go-NoGo task. Neuroscience 156: 450-5.

Packard MG, Cahill L (2001) Affective modulation of multiple memory systems. Curr Opin Neurobiol 11: 752-56.

Palermo R, Schmalzl L, Mohamed A, Bleasel A, Miller L (2007) The impact of unilateral amygdala lesions on the detection of brief backward-masked fearful facial expressions.

Perception 36: ECVP Abstract Supplement. 
Pelphrey KA, Sasson NJ, Reznick JS, Paul G, Goldman BD, Piven J (2002) Visual scanning of faces in autism. J Autism Dev Disord 32: 249-61.

Pelphrey KA, Singerman JD, Allison T, McCarthy G (2003) Brain activation evoked by perception of gaze shifts: The influence of context. Neuropsychologia 41: 156-70.

Pelphrey KA, Viola RJ, McCarthy G (2004) When strangers pass: processing of mutual and averted gaze in the superior temporal sulcus. Psychol Sci 15: 598-603.

Pelphrey KA, Morris JP, Michelich CR, Allison T, McCarthy G (2005) Functional anatomy of biological motion perception in posterior temporal cortex: an fMRI study of eye, mouth, and hand movements. Cereb Cortex 15: 1866-76.

Pessoa L, McKenna M, Gutierrez E, Ungerleider LG (2002) Neural processing of emotional faces requires attention. Proc Natl Acad Sci U S A 99: 11458-63.

Phan KL, Wager T, Taylor SF, Liberzon I, (2002) Functional neuroanatomy of emotion: a metaanalysis of emotion activation studies in pet and fMRI. Neuroimage 16: 331-48.

Phelps EA, LeDoux JE (2005) Contributions of the amygdala to emotion processing: from animal models to human behavior. Neuron 48: 175-87.

Phillips ML, Drevets WC, Rauch SL, Lane R (2003) The neurobiology of emotion perception: The neural basis of normal emotion perception. Biol Psychiatry 54: 504-14.

Phillips ML, Williams LM, Heining M, Herba CM, Russell T, Andrew C, Bullmore ET, Brammer MJ, Williams SC, Morgan M, Young AW, Gray JA (2004) Differential neural responses to overt and covert presentations of facial expressions of fear and disgust. Neuroimage 21: 1484-96.

Pierce K, Muller RA, Ambrose J, Allen G, Courchesne E (2001) Face processing occurs outside the fusiform 'face area' in autism: evidence from functional MRI. Brain 124: 2059:73.

Pitkänen A, Savander V, LeDoux JE (1997) Organization of intraamygdaloid circuitries in the rat: an emerging framework for understanding functions of the amygdala. Trends Neurosci 20: 517-23.

Pitkänen A (2000) Connectivity of the rat amygdaloid complex. Aggleton JP (Ed.) In: The amygdala: a functional analysis (31-115) Oxford: Oxford Univ. Press.

Poremba A, Gabriel M (1999) Amygdala neurons mediate acquisition but not maintenance of instrumental avoidance behavior in rabbits. J Neurosci 19: 9635-41.

Price JL, Russchen FT, Amaral DG (1987) The Limbic Region. II: The Amygdaloid Complex. New York: Elsevier Science. 
Puce A, Allison T, Gore JC, McCarthy G (1995) Face-sensitive regions in human extrastriate cortex studied by functional MRI. J Neurophysiol 74: 1192-9.

Puce A, Allison T, Asgari M, Gore JC, McCarthy G (1996) Differential sensitivity of human visual cortex to faces, letterstrings, and textures: a functional magnetic resonance imaging study. J Neurosci 16: 5205-15.

Puce A, Allison T, Bentin S, Gore JC, McCarthy G (1998) Temporal cortex activation in human subjects viewing eye and mouth movements. J Neurosci 18: 2188-99.

Puce A, Allison T, McCarthy G (1999) Electrophysiological studies of human face perception. III: effects of top-down processing on face-specific potentials. Cereb Cortex 9: 445-58.

Puce A, Smith A, Allison T (2000) ERPs evoked by viewing facial movements. Cog Neuropsych 17:221-39.

Puce A, Syngeniotis A, Thompson JC, Abbott DF, Wheaton KJ, Castiello U (2003) The human temporal lobe integrates facial form and motion:evidence from fMRI and ERP studies.

Neuroimage 19: 861-9.

Ricciardelli P, Bricolo E, Aglioti SM, Chelazzi L (2002) My eyes want to look where your eyes are looking: exploring the tendency to imitate another individual's gaze. Neuroreport 13: 225964.

Rhodes G (1985) Lateralized processes in face recognition. Br J Psychol 76: 249-71.

Richer JM, Cross RG (1976) Gaze aversion in autistic and normal children. Acta Psychiatr Scand 53: 193-210.

Ristic J, Mottron L, Friesen CK, Iarocci G, Burack JA, Kingstone A (2005) Eyes are special but not for everyone: the case of autism. Brain Res Brain Res Cogn 24: 715-8.

Rolls ET, Rolls BJ (1973) Altered food preferences after lesions in the basolateral region of the amygdala. J Comp Physiol Psychol 83: 248-59.

Rolls ET, Tovee MJ (1994) Processing speed in the cerebral cortex and the neurophysiology of visual masking. Proc Biol Sci 257: 9-15.

Rolls ET (2000) Neurophysiology and functions of the primate amygdala, and the neural basis of emotion. Aggleton, JP (Ed.) In: The amygdala: a functional analysis. (pp 479-508) Oxford: Oxford University Press.

Roozendaal B, Koolhaas JM, Bohus B (1993) The central amygdala is involved in conditioning but not in retention of active and passive shock avoidance in male rats. Behav Neural Biol 59: 143-9. 
Sabatinelli D, Bradley MM, Fitzsimmons JR, Lang PJ (2005) Parallel amygdala and inferotemporal activation reflect emotional intensity and fear relevance. Neuroimage 24: 126570 .

Sadr J, Jarudi I, Sinha P (2003) The role of the eyebrows in face recognition. Perception 32: 285-93.

Sah P, Faber ES, Lopez de Armentia M, Power J (2003) The amygdaloid complex: anatomy and physiology. Physiol Rev 83: 803-34.

Sams M, Hietanen JK, Hari R, Ilmoniemi RJ, Lounasmaa OV (1997) Face-specific responses from the human inferior occipito-temporal cortex. Neuroscience 77: 49-55.

Sander D, Grafman J, Zalla T, (2003) The human amygdala: an evolved system for relevance detection. Rev Neurosci 14: 303-16.

Sato W, Kochiyama T, Yoshikawa S, Naito E, Matsumura M (2004) Enhanced neural activity in response to dynamic facial expressions of emotion: an fMRI study. Brain Res Cogn Brain Res 20: 81-91.

Schmolck H, Squire LR, (2001) Impaired perception of facial emotions following bilateral damage to the anterior temporal lobe. Neuropsychology 15: 30- 38.

Schneider F, Habel U, Kessler C, Salloum JB, Posse S (2000) Gender differences in regional cerebral activity during sadness. Hum Brain Mapp 9: 226-38.

Schultz RT, Gauthier I, Klin A, Fulbright RK, Anderson AW, Volkmar F, Skudlarski P, Lacadie C, Cohen DJ, Gore JC (2000) Abnormal ventral temporal cortex activity during face discrimination among individuals with autism and Asperger syndrome. Arch Gen Psychiatry 57: $331-40$.

Schultz RT, Grelotti DJ, Klin A, Kleinman J, Van der Gaag C, Marois R, Skudlarski P (2003) The role of the fusiform gyrus in social cognition: implications for the pathbiology of autism. Philos Trans R Soc Lond B Biol Sci 358: 415-27.

Schwarzer G (2000) Development of face processing: the effect of face inversion. Child Dev 71: 391-401.

Schyns PH, Bonnar L, Gosselin F (2002) Show me the features! Understanding recognition from the use of visual information. Psychol Sci 13: 402-9.

Schyns PG, Petro LS, Smith ML (2007) Dynamics of visual information integration in the brain for categorizing facial expressions. Curr Bio 17: 1580-5. 
Sergent J, Bindra D (1981) Differential hemispheric processing of faces: methodological considerations and reinterpretation. Psychol Bull 89: 541-54.

Serrano JM, Iglesias J, Loeches A (1992) Visual discrimination and recognition of facial expressions of anger, fear and surprise in 4- and 6-month-old infants. Dev Psychobiol 25: 41125 .

Shulman GL, d'Avossa G, Tansy AP, Corbetta M (2002) Two attentional processes in the parietal lobe. Cereb Cortex 12: 1124-31.

Smith ML, Cottrell GW, Gosselin F, Schyns PG (2005) Transmitting and decoding facial expressions. Psychol Sci 16: 184-9.

Spezio ML, Adolphs R, Hurley RS, Piven J (2006) Abnormal use of facial information in highfunctioning autism. J Autism Dev Disord 37: 929-39.

Spezio ML, Adolphs R, Hurley RS, Piven J (2007) Analysis of face gaze in autism using "Bubbles". Neuropsychologia 45: 144-51.

Stephan BC, Breen N, Caine D (2006) The recognition of emotional expression in prosopagnosia: decoding whole and part faces. J Int Neuropsych Soc 12: 884-95.

Swanson LW, Petrovich GD (1998) What is the amygdala? Trends in Neurosciences 21: 323-31.

Talairach J, Tournoux P (1988) A co-planar stereotactic atlas of the human brain. Thieme: Stuggart.

Tanaka JW, Farah MJ (1993) Parts and wholes in face recognition. Q J Exp Psychol A 46: 22545.

Thomas KM, Drevets WC, Whalen PJ, Eccard CH, Dahl RE, Ryan ND, Casey BJ (2001) Amygdala response to facial expressions in children and adults. Biol Psychiatry 49: 309-16.

Thompson KG, Bichot NP, Schall JD (1997) Dissociation of visual discrimination from saccade programming in macaque frontal eye field. J Neurophysiol 77: 1046-1050.

Thompson JC, Clarke M, Stewart T, Puce A (2005) Configural processing of biological motion in human superior temporal sulcus. J Neurosci 25: 9059-66.

Todorv A, Engell AD (2008) The role of the amygdala in implicit evaluation of emotionally neutral faces. Soc Cogn Affect Neurosci 3: 303-12.

Tong F, Nakayama K, Moscovitch M, Weinrib O, Kanwisher N (2000) Response properties of the human fusiform face area. Cog Neuropsychology 17: 257-79. 
Treit D, Menard J (1997) Dissociations among the anxiolytic effects of septal, hippocampal, and amygdaloid lesions. Behav Neurosci 111: 653-58.

van der Gaag C, Minderaa RB, Keysers C (2007) The BOLD signal in the amygdala does not differentiate between dynamic facial expressions. Soc Cogn Affect Neuroci 2: 93-103.

Vignal JP, Chauvel P, Halgren E (2000) Localized face-processing by the human prefrontal cortex: Stimulation-evoked hallucinations of faces. Cog Neuropsychology 17: 281-91.

Vuilleumier P, Schwartz S (2001) Emotional facial expressions capture attention. Neurology 56: 153-8.

Vuilleumier P, Armony JL, Driver J, Dolan RJ (2001) Effects of attention and emotion on face processing in the human brain: an event-related fMRI study. Neuron 30: 829-41.

Vuilleumier P, Armony JL, Driver J, Dolan RJ. (2003) Distinct spatial frequency sensitivities for processing faces and emotional expressions. Nat Neurosci. 6: 624-31.

Vuilleumier P, Richardson MP, Armony JL, Driver J, Dolan RJ (2004). Distant influences of amygdala lesion of visual cortical activation during emotional face processing. Nat Neurosci 7: 1271-8.

Vuilleumier P (2005) How brains beware: neural mechanisms of emotional attention. Trends Cogn Sci 9: 585-94.

Vuilleumier P, Driver J (2007) Modulation of visual processing by attention and emotion: windows on causal interactions between human brain regions. Philos Trans $R$ Soc Lond B Biol Sci 362: 837-55.

Wager TD, Phan KL, Liberzon I, Taylor SF (2003) Valence, gender, and lateralization of functional brain anatomy in emotion: a metaanalysis of findings from neuroimaging.

Neuroimage 19: 513-31.

Watanabe S, Kakigi R, Koyama S, Kirino E (1999a) It takes longer to recognize the eyes than the whole face in humans. Neuroreport 10: 2193-8.

Watanabe S, Kakigi R, Koyama S, Kirino E (1999b) Human face perception traced by magnetoand electro-encephalography. Brain Res Brain Res Cogn 8: 125-42.

Whalen PJ, Rauch SL, Etcoff NL, McInerney SC, Lee MB, Jenike MA (1998) Masked presentations of emotional facial expressions modulate amygdala activity without explicit knowledge. J Neurosci 18: 411-8.

Whalen PJ, Shin LM, McInerney SC, Fischer H, Wright CI, Rauch SL (2001) A functional MRI study of human amygdala responses to facial expressions of fear versus anger. Emotion 1: 70-83. 
Whalen PJ, Kagan J, Cook RG, Davis FC, Kim H, Polis S, McLaren DG, Somerville LH, McLean AA, Maxwell JS, Johnstone T (2004) Human amygdala responsivity to masked fearful eye whites. Science 306: 2061.

Whalen PJ, Phelps EA (2009) The human amygdala. New York: The Guildford Press.

Wheaton KJ, Pipingas A, Silberstein RB, Puce A (2001) Human neural response elicited to observing the actions of others. Vis Neurosci 18: 401-6.

Wheaton KJ, Thompson JC, Syngeniotis A, Abbott DF, Puce A (2004) Viewing the motion of human body parts activates different regions of premotor, temporal, and parietal cortex.

Neuroimage 22: 277-288.

Wicker B, Michel F, Henaff MA, Decety J (1998) Brain regions involved in the perception of gaze: a PET study. Neuroimage 8: 221-7.

Williams MA, Morris AP, McGlone F, Abbott DF, Mattingley JB (2004) Amygdala responses to fearful and happy facial expressions under conditions of binocular suppression. J Neurosci 24: 2898-904.

Wilson FA, Rolls ET (1993) The effects of stimulus novelty and familiarity on neuronal activity in the amygdala of monkeys performing recognition memory tasks. Exp Brain Res 93: 367-82.

Winston JS, O'Doherty J, Dolan RJ (2003) Common and distinct neural responses during direct and incidental processing of multiple facial emotions. Neuroimage 20: 84-97.

Woods RP, Cherry SR, Mazziotta JC (1992) Rapid automated algorithm for aligning and reslicing PET images. J Comput Assist Tomogr 16: 620-33.

Woods RP, Grafton ST, Holmes CJ, Cherry SR, Mazziotta JC (1998) Automated image registration: I. General methods and intrasubject, intramodality validation. J Comput Assist Tomogr 22: 139-52.

Worsley KJ, Marrett S, Neelin P, Vandal AC, Friston KJ, Evans AC (1996) A unified statistical approach for determining significant signals in images of cerebral activation. Hum Brain Mapp 4: 58-73.

Wright CI, Fischer H, Whalen PJ, McInerney SC, Shin LM, Rauch SL (2001) Differential prefrontal cortex and amygdala habituation to repeatedly presented emotional stimuli. Neuroreport 12: 379-83.

Yang TT, Menon V, Eliez S, Blasey C, White CD, Reid AJ, Gotlib IH, Reiss AL (2002) Amygdalar activation associated with positive and negative facial expressions. Neuroreport 13: $1737-41$. 
Yarbus A (1967) Eye movements and vision. New York: Plenum Press.

Yin R (1969) Looking at upside down faces. J Exp Psychol 81: 141-5.

Young AW, Aggleton JP, Hellawell DJ, Johnson M, Brooks P, Hanley JR (1995) Face processing impairments after amygdalotomy. Brain 188:5-24.

Young AW, Hellawell DJ, Van De Wal C, Johnson M (1996) Facial expression processing after amygdalotomy. Neuropsychologia 34: 31-9.

Zald DH, Pardo JV (2002) The neural correlates of aversive auditory stimulation. Neuroimage 16: 746-53.

Zald DH (2003) The human amygdala and the emotional evaluation of sensory stimuli. Brain Res Brain Res Rev 41: 88-123.

Zola-Morgan S, Squire LR, Alvarez_Royo P, Clower RP (1991) Independence of memory functions and emotional behavior: separate contributions of the hippocampal formation and the amygdala. Hippocampus 1: 207-20. 


\section{Curriculum Vitae}

\author{
Education \\ Current
}

1996-2001
$\mathrm{PhD}$ in Neuroscience, Center for Advanced Imaging, West Virginia University, Morgantown, WV

Research interests: functional neuroimaging, social cognition, biological motion, emotion, neural processing of faces

Bachelor of Science in Biological Sciences, West Virginia University, Morgantown, WV Minor: English, Anthropology

\section{Research}

- fMRI in human subjects

- fMRI data analysis using Matlab, SPM, FSL, AFNI

- Pupillometry and gaze monitoring data collection and analysis

- Experience with EEG, TMS

\section{Publications}

2008 Thompson JC, Hardee JE. The first time ever I saw your face. Trends in Cognitive Science. 12, 283-4

Hardee JE, Thompson JC, Puce A. The left amygdala knows fear: laterality in the amygdala response to fearful eyes. Social Cognitive and Affective Neuroscience. 3, 47-54

2007 Thompson JC, Hardee JE, Panayiotou A, Crewther D, Puce A. Common and distinct brain activation to viewing dynamic sequences of face and hand movements. Neuroimage. 37, 966-73

\section{Conference Abstracts and Presentations}

2008 Hardee JE, Thompson JC, Prostko AL, Brefczysnki-Lewis JA, Puce A. Differential amygdala activation to emotional eyes and eyebrows. SFN Annual Scientific Meeting. \#786.10

Prostko AL, Hardee JE, Brefczynski-Lewis J, Pollard SE, Puce A. Differential brain activation patterns to interpreting social scenes under different viewing contexts. SFN Annual Scientific Meeting. \#789.3

Puce A, McNeely M, Carrick O, Berrebi M, Epling J, Thompson JC, Hardee JE, Zellner L, Brefczynski-Lewis J. N170 amplitude reflects the seen number of faces irrespective of low-level stimulus variables. OHBM Annual Scientific Meeting. \#714

2006 Hardee JE, Thompson JC, Puce A. Hemispheric differences in the amygdala response to gaze shifts and emotional eyes. Platform talk and poster. OHBM Annual Scientific Meeting. \#54.

Thompson, JC, Hardee JE, Panayiotou A, Crewther D, Puce A. The superior temporal sulcus is a generic biological motion processor. OHBM Annual Scientific Meeting. \#450 


\section{Book Chapters}

2008 Hardee JE, Thompson JC. Neuroscience in human communication: verbal and nonverbal. In Polack EP, Richmond VP, McCroskey JC (Eds), Applied interpersonal communication for health professionals (2008)

\section{Honors and Awards}

2006 Travel Award; Organization for Human Brain Mapping Annual Scientific Meeting

Fellow; Summer Institute in Cognitive Neuroscience; Dartmouth; Hanover, NH

Fellow; PENS/Hertie Winter School: Brain Basis of Social Interaction; Kitzbuhel, Austria

\section{Teaching}

2009 Coursework: Teaching practicum (PSIO 790), Dr. Mark Paternostro, West Virginia University

Tutor; Fundamentals of physiology (PHYS 241), coordinator Dr. Stan Yokota, West Virginia University

2008 Tutor; Fundamentals of physiology (PHYS 241), coordinator Dr. Stan Yokota, West Virginia University

2007 Lecturer; Advanced Neuroscience (NBAN 795), coordinator Dr. Aina Puce West Virginia University

2006 Lecturer; Fundamentals of Integrative Systems (CCMD 793A), coordinator Dr. Albert Berrebi, West Virginia University

\section{Professional Memberships and Committees}

- Organization for Human Brain Mapping (2006-present)

- Cognitive Neuroscience Society (2007-present)

- Society for Neuroscience (2008-present)

- Member of WVU Graduate Program Admissions Committee: 2006-2007 